\title{
Enhanced prediction and prevention of drug-induced torsades de pointes
}

Citation for published version (APA):

Johnson, D. M. (2013). Enhanced prediction and prevention of drug-induced torsades de pointes.

[Doctoral Thesis, Maastricht University]. BOXPress. https://doi.org/10.26481/dis.20130308dj

Document status and date:

Published: 01/01/2013

DOI:

10.26481/dis.20130308dj

Document Version:

Publisher's PDF, also known as Version of record

\section{Please check the document version of this publication:}

- A submitted manuscript is the version of the article upon submission and before peer-review. There can be important differences between the submitted version and the official published version of record.

People interested in the research are advised to contact the author for the final version of the publication, or visit the DOI to the publisher's website.

- The final author version and the galley proof are versions of the publication after peer review.

- The final published version features the final layout of the paper including the volume, issue and page numbers.

Link to publication

\footnotetext{
General rights rights.

- You may freely distribute the URL identifying the publication in the public portal. please follow below link for the End User Agreement:

www.umlib.nl/taverne-license

Take down policy

If you believe that this document breaches copyright please contact us at:

repository@maastrichtuniversity.nl

providing details and we will investigate your claim.
}

Copyright and moral rights for the publications made accessible in the public portal are retained by the authors and/or other copyright owners and it is a condition of accessing publications that users recognise and abide by the legal requirements associated with these

- Users may download and print one copy of any publication from the public portal for the purpose of private study or research.

- You may not further distribute the material or use it for any profit-making activity or commercial gain

If the publication is distributed under the terms of Article $25 \mathrm{fa}$ of the Dutch Copyright Act, indicated by the "Taverne" license above, 
Enhanced Prediction and Prevention of Drug-Induced Torsades de Pointes 
(C) Daniel Johnson, Maastricht - Luik 2012

ISBN: $\quad 978-90-8891-573-4$

Layout: Daniel Johnson

Production: Uitgeverij BOXPress || Proefschriftmaken.nl

All rights reserved. No part of this thesis may be reproduced, stored in a retrieval system or transmitted in any form or by means, without the permission in writing from the author, or, when appropriate, of the publishers of the publications.

Cover Illustration:

(c) René Magritte, The Blood of the World c/o Pictoright Amsterdam 2012 


\section{Enhanced Prediction and Prevention of Drug-Induced Torsades de Pointes}

\section{PROEFSCHRIFT}

Ter verkrijging van de graad van doctor aan de Universiteit Maastricht

op gezag van de Rector Magnificus, Prof. Dr. L.L.G. Soete, volgens het besluit van het College van Decanen, in het openbaar te verdedigen op

vrijdag 8 maart 2013 om 10.00 uur

door

\section{Daniel Michael J ohnson}

Geboren op 13 augustus 1979 te Leeds, Verenigd Koninkrijk 


\section{Promotor}

Prof. Dr. H.J.G.M. Crijns

\section{Copromotoren}

Dr. P.G.A. Volders

Dr. N. Abi-Gerges (United Kingdom)

\section{Beoordelingscommissie}

Prof. Dr. U. Schotten (Voorzitter)

Prof. Dr. A.P.M. Gorgels

Prof. Dr. T.G. Hammond (University of Liverpool, United Kingdom)

Prof. Dr. M.A. Vos (Universitair Medisch Centrum, Utrecht, The Netherlands)

Prof. Dr. T. Unger

Financial support by 'Stichting Hartsvrienden RESCAR Maastricht' for publication of this thesis is gratefully acknowledged.

Additional support was granted by Astrazeneca R\&D, Alderley Park, UK. 
For Hannah 



\section{Table of Contents}

Chapter 1 General Introduction 9

Chapter 2 Measurement of Action Potential Generation in Isolated 31

Canine Left Ventricular Midmyocardial Myocytes

$\begin{array}{lll}\text { Chapter } 3 & \mathrm{I}_{\mathrm{Ks}} \text { Restricts Excessive Beat-to-Beat Variability of } & \mathbf{6 5}\end{array}$

$\begin{array}{lll}\text { Chapter } 4 & \text { Diastolic Spontaneous Calcium Release from the }\end{array}$

Sarcoplasmic Reticulum Increases Beat-to-Beat

Repolarization Variability in Canine Ventricular Myocytes

after $\beta$-Adrenergic Stimulation

Chapter $5 \quad$ Interventricular Differences in $\beta$-Adrenergic Responses $\quad 131$

in the Canine Heart: Role of Phosphodiesterases

$\begin{array}{lll}\text { Chapter } 6 & \text { Reduced Ventricular Proarrhythmic Potential of the } & 157\end{array}$

Novel Combined Ion-Channel Blocker AZD1305 versus

Dofetilide in Dogs With Remodeled Hearts

Chapter 7 The Electro-Mechanical Window in Anaesthetized

183

Guinea-Pigs: A New Marker for Torsades de Pointes

Risk Screening

Chapter 8 General Discussion

Summary / Samenvatting $\quad 231$

Acknowledgements / Dankwoord $\quad 241$

Curriculum Vitae $\quad 247$

$\begin{array}{ll}\text { Publications } & 249\end{array}$ 

Chapter 1

\section{General I ntroduction}

Daniel M Johnson • Najah Abi-Gerges • Paul GA Volders 


\subsection{Background}

Since the first case report of syncope during initiation of quinidine therapy in the $1920 \mathrm{~s}^{1}$ until the most recent reports of drug withdrawals, drug-induced arrhythmias have been high on the agenda of the pharmaceutical industry, regulatory agencies, healthcare professionals, and patients. Up until today, 14 drugs have been withdrawn from the market due to this deleterious side-effect (Table 1). In 1997, a pivotal case report ${ }^{2}$ triggered public awareness regarding the risks associated with drug-induced arrhythmia. In this publication, a 59 year-old man, who was otherwise healthy presented to the emergency department after complaining of dizziness and shortness of breath whilst playing handball. When the patient's electrocardiogram was recorded, episodes of 'torsades de pointes' (TdP) were found to underlie his symptoms. The patient had been taking the antihistamine, terfenadine, the previous day for nasal congestion due to hay fever. During clinical evaluation, the possibilities that the arrhythmia were caused by liver disease, ischemia, myocardial infarction or electrolyte imbalances were ruled out, and it was considered likely that terfenadine, a drug available without prescription and taken without concomitant other drugs, was the likely trigger for the arrhythmia. In early 1998, terfenadine was withdrawn from the market due to its potential to lead to TdP-type arrhythmias.

Terfenadine was not the first marketed drug to be withdrawn due to cardiovascular safety concerns (see Table 1), but its story has become extremely important for drug development. It illustrated that compounds with even small risks of very serious side-effects, such as drug-induced TdP, could upset the risk-benefit balance of any new chemical entity. Recent work has discussed the possibilities that terfenadine-induced dysrhythmias may be due to its ability to cause ventricular fibrillation rather than $\mathrm{TdP}^{3}$.

Safety pharmacology studies play a significant role in guiding the pharmaceutical industry in preventing unsafe agents, such as terfenadine, to reach the patient population. In addition, this discipline is involved in providing valuable insights into the mechanisms of potential adverse effects of drug candidates. Although the term 'safety pharmacology' was used prior to the inception of the ICH S7A document in 2001, where the core battery studies of safety pharmacology are described, it was in this document that the term was defined. These guidelines provide a basis for the preclinical safety studies, in three major areas (cardiovascular, respiratory and the central nervous system), that need to be performed before a new chemical entity can be tested in human studies. 
Table 1

\begin{tabular}{|c|c|c|c|}
\hline \multicolumn{1}{|c}{ Drug } & $\begin{array}{c}\text { Year of } \\
\text { Introduction }\end{array}$ & \multicolumn{1}{c}{ Therapeutic Area } & $\begin{array}{c}\text { Year of } \\
\text { Withdrawal }\end{array}$ \\
Prenylamine & 1960 s & Antianginal & 1988 \\
Lidoflazine ${ }^{*}$ & 1979 & Antianginal & 1989 \\
Terodiline & 1986 & Antianginal / Urinary incontinence & 1991 \\
Terfenadine & 1982 & Antihistamine & 1998 \\
Sertindole $*$ & 1996 & Antipsychotic & 1998 \\
Astemizole & 1986 & Antihistamine & 1999 \\
Grepafloxacin & 1997 & Antibiotic & 1999 \\
Cisapride & 1988 & Gastric prokinetic & 2000 \\
Droperidol & 1960 s & Tranquillizer / Analgesic & 2001 \\
Levacetylmethadol & 1997 & Methadone substitution & 2001 \\
Dofetilide ${ }^{\#}$ & 1999 & Class-III antiarrhythmic drug for & 2003 \\
& & atrial fibrillation & \\
Thioridazine & 1960 s & Antipsychotic & 2005 \\
Clobutinol & 1960 s & Antitussive & 2007 \\
Dextropropoxyphene $* *$ & 1960 s & Opioid analgesic & 2009 \\
\hline
\end{tabular}

* Re-introduced later following re-evaluation of risk-benefit; ** In addition to QT-liability, safety in overdose was also an issue. " Withdrawn by the sponsor for commercial reasons, European market.

Table 1: Drugs withdrawn from the major markets of the world as a result of their potential for QT prolongation and/or TdP (Courtesy of Dr Rashmi R Shah ${ }^{4}$ ).

Drug attrition rates remain high, and this is one of the major reasons why the costs of drug development are astronomical. The major reasons for drug attrition are illustrated in Figure $\mathbf{1}$ as was initially shown in a study of Kola and Landis ${ }^{5}$. Whilst efficacy of compounds at drug targets has increased over the years, as have pharmacokinetic and bioavailability issues, attrition due to safety is actually becoming more of an issue. Therefore, a large unmet need clearly exists for the improvement of these safety issues at the early phases of the drug discovery process. 


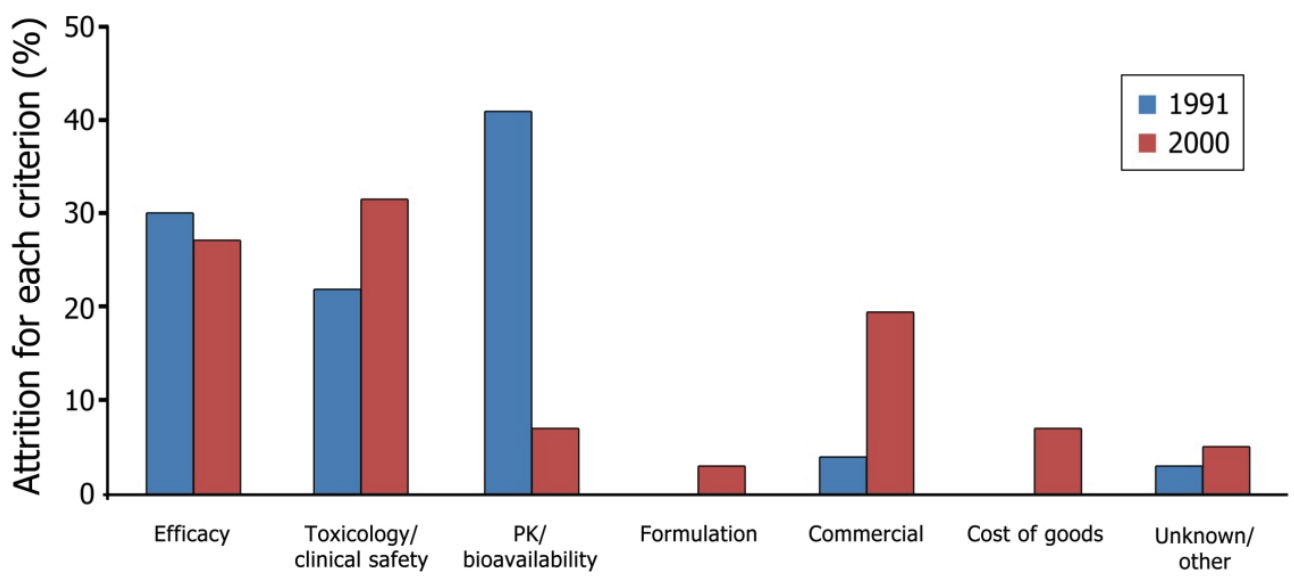

Figure 1: Reasons for drug attrition in 1991 and 2000. PK, Pharmacokinetics. Modified from Kola and Landis, 2004. Over the 10-year period safety, both non-clinical toxicology and clinical, remain a major cause of drug attrition accounting for approximately $30 \%$ of all drug discontinuation (Adapted from Laverty et al.; 2011) ${ }^{6}$.

From the three major areas that are studied under the auspices of the ICH S7A guidelines, cardiovascular risk, and particularly arrhythmogenic risk, remains one of the major reasons for halting of preclinical programs as well as withdrawal of compounds from the market. Figure 2 illustrates the prevalence and occurrence of safety liabilities relating to the major organ systems at all stages of the drug development process. Interestingly, the only stage of this process where cardiovascular events are not the major cause of attrition is during phase 1 , where healthy subjects are exposed to the new chemical entity. One of the major reasons for this could be the fact that cardiac side-effects are more likely to show up in diseased hearts that have undergone remodelling, e.g., due to pressure or volume overload. Patients showing these characteristics are more likely to show up later on in the drug development process, and ultimately may be the desired target population. This underscores the importance of screening compounds for cardiac side-effects in disease models (see section 1.3). Figure $\mathbf{3}$ shows the major adverse events in the cardiovascular field that have been reported to the Food and Drug Administration (FDA) since 1969. Although the incidence of arrhythmia may be relatively high due to increased scrutiny of drug-induced arrhythmias over the last decade, these data illustrate the importance of arrhythmia as an unwanted cardiovascular side-effect. In addition, according to the data of Shah (2006) ${ }^{7}$, QT prolongation was the reason for about one-third of all drug withdrawals between 1990 and 2006. Despite the fact that prolongation of the QT interval in itself is not a safety issue, it is currently the most widely used risk marker for TdP arrhythmias. 


\begin{tabular}{|c|c|c|c|c|c|c|}
\hline Phase & 'Nonclinical' & Phase I & Phase I-III & $\begin{array}{l}\text { Phase III/ } \\
\text { Marketing }\end{array}$ & $\begin{array}{c}\text { Post- } \\
\text { Marketing }\end{array}$ & $\begin{array}{c}\text { Post- } \\
\text { Marketing }\end{array}$ \\
\hline Information: & $\begin{array}{l}\text { Causes of } \\
\text { attrition }\end{array}$ & Serious ADRs & $\begin{array}{l}\text { Causes of } \\
\text { attrition }\end{array}$ & ADRs on label & Serious ADRs & $\begin{array}{l}\text { Withdrawal from } \\
\text { sale }\end{array}$ \\
\hline Source: & $\operatorname{Car}(2006)$ & $\begin{array}{c}\text { Sibille et al. } \\
\text { (1998) }\end{array}$ & $\begin{array}{l}\text { Olson et al. } \\
(2000)\end{array}$ & BioPrint $(2006)$ & $\begin{array}{l}\text { Budnitz et al. } \\
\text { (2006) }\end{array}$ & $\begin{array}{c}\text { Stevens \& Baker } \\
(2008)\end{array}$ \\
\hline Sample size: & 88 CDs stopped & 1,015 subjects & 82 CDs stopped & 1,138 drugs & 21,298 patients & 47 drugs \\
\hline Cardiovascular: & $27 \%$ & $9 \%$ & $21 \%$ & $36 \%$ & $15 \%$ & $45 \%$ \\
\hline Hepatotoxicity: & $8 \%$ & $7 \%$ & $21 \%$ & $13 \%$ & $0 \%$ & $32 \%$ \\
\hline Haematology/BM: & $7 \%$ & $2 \%$ & $4 \%$ & $16 \%$ & $10 \%$ & $9 \%$ \\
\hline Nervous system: & $14 \%$ & $28 \%$ & $21 \%$ & $67 \%$ & $39 \%$ & $2 \%$ \\
\hline Immunotox; photosensitivity: & $7 \%$ & $16 \%$ & $11 \%$ & $25 \%$ & $34 \%$ & $2 \%$ \\
\hline Gastrointestinal: & $3 \%$ & $23 \%$ & $5 \%$ & $67 \%$ & $14 \%$ & $2 \%$ \\
\hline Reprotox: & $13 \%$ & $0 \%$ & $1 \%$ & $10 \%$ & $0 \%$ & $2 \%$ \\
\hline Musculoskeletal: & $4 \%$ & $0 \%$ & $1 \%$ & $28 \%$ & $3 \%$ & $2 \%$ \\
\hline Respiratory: & $2 \%$ & $0 \%$ & $0 \%$ & $32 \%$ & $8 \%$ & $2 \%$ \\
\hline Renal: & $2 \%$ & $0 \%$ & $9 \%$ & $19 \%$ & $2 \%$ & $0 \%$ \\
\hline Genetic tox: & $5 \%$ & $0 \%$ & $0 \%$ & $0 \%$ & $0 \%$ & $0 \%$ \\
\hline Carcinogenicity: & $3 \%$ & $0 \%$ & $0 \%$ & $1 \%$ & $0 \%$ & $0 \%$ \\
\hline other: & $0 \%$ & $0 \%$ & $4 \%$ & $16 \%$ & $2 \%$ & $2 \%$ \\
\hline
\end{tabular}

The various toxicity domains have been ranked first by contribution to products withdrawn from sale, then by attrition during clinical development.

Figure 2: Prevalence of safety liabilities relating to the major organ systems. At nearly all stages of drug development, cardiovascular liability causes the highest risk of safety issues (From Redfern et al.; 2010) ${ }^{8}$.

In 2005, the ICH introduced an additional guideline, ICH S7B, specifically addressing the issues of non-clinical evaluation of human pharmaceuticals on ventricular repolarization. In this guideline various recommendations were made, including an in-vitro screen to evaluate the effects of the compound on the rapid component of the delayed rectifier potassium current $\left(\mathrm{I}_{\mathrm{Kr}}\right)$ besides an in-vivo QT study. Later that same year, the clinical counterpart to ICH S7B, ICH E14, was also introduced. This guideline advocates the use of a thorough QT study in a randomized, double-blinded manner in healthy volunteers for all new chemical entities. 


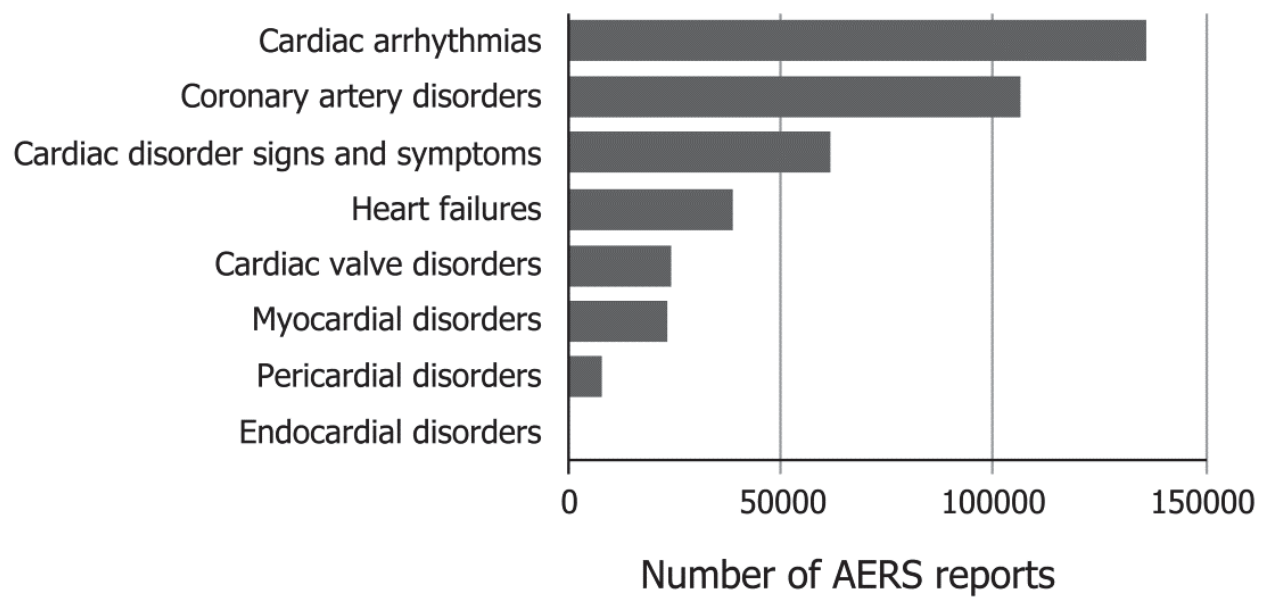

Figure 3: Cumulative cardiac adverse events (AEs) reported to the US Food and Drug Administration (FDA) Adverse Event Reporting System (AERS) since 1969. Cardiac arrhythmias remain the most reported adverse effect in the cardiovascular field (From Laverty et al.; 2011) ${ }^{5}$.

The limitations of these guidelines are widely recognized. Among these, QT prolongation per se does not necessarily lead to arrhythmias ${ }^{9,} 10$ (see section 1.3). Moreover, arrhythmia initiation is not a static process and many factors interact, some of which remain unknown to date. With regard to the $\mathrm{I}_{\mathrm{Kr}}$ channel, when a compound blocks this channel, the same compound may not cause TdP in vivo. Thus, it is very likely that efficacious compounds are discarded early in the drugdevelopment process due to inhibitory effects on $\mathrm{I}_{\mathrm{Kr}}$, whereas simultaneous actions on other ion channels or proteins may offset the $I_{\mathrm{kr}}$ effects, rendering the compounds safe for in-vivo use. Alternatively, there are examples of compounds that may cause arrhythmias without having major effects on $\mathrm{I}_{\mathrm{Kr}}\left(\right.$ e.g., JNJ303 ${ }^{11}$ ), and compounds that can increase repolarization duration while having minimal effects on $\mathrm{I}_{\mathrm{Kr}}\left(\mathrm{e} . \mathrm{g}\right.$. , alfusozin ${ }^{12}$ ).

As attrition rates and cardiac side-effects remain relatively high, there is a pressing need for better understanding of the mechanisms involved in TdP. Once these mechanisms are uncovered, this will allow for better prediction (via more predictive (surrogate) markers) and prevention (via novel targets) of this arrhythmia.

\subsection{The Ventricular Action Potential and Arrhythmogenesis}

To understand the mechanisms of arrhythmogenesis, it is important to comprehend the complexity of the normal electrical activity of the heart and single cardiac cells. Each normal heart beat is initiated by an electrical impulse that originates in special pacemaker cells in the sinus node. From here, electrical activity spreads via the atria and conduction system, ultimately activating the ventricular myocytes. 
Activation of these cells leads to an action potential configuration as shown in Figure 4. Since the first recordings of action potentials in canine Purkinje fibres in the 1950's by Draper and Weidmann ${ }^{13}$, we have come to understand the ionic basis of the cardiac action potential in great detail. The ventricular action potential is made up of complex interactions between both inward and outward ion movements across the membrane of myocytes causing both depolarization and repolarization. In phase 0 there is an inward movement of sodium ions $\left(\mathrm{I}_{\mathrm{Na}}\right)$, which leads to rapid depolarization of the cell. During phase 1 rapid repolarization occurs via the transient outward potassium current $\left(\mathrm{I}_{\mathrm{TO}}\right)$. In some species, calcium-activated chloride current $\left(\mathrm{I}_{\mathrm{CI}(\mathrm{Ca})}\right)$ also contributes. Following this, there is a tightly controlled balance of both inward and outward currents that determines the duration of the plateau phase of the action potential (phase 2). During this time period, the outward currents are carried by a number of potassium channels, including the rapid and slow delayed rectifier currents ( $I_{\mathrm{Kr}}$ and $\mathrm{I}_{\mathrm{Ks}}$, respectively) and the sodium-potassium pump ( $\mathrm{I}_{\mathrm{NaK}}$ ). Inward current is mainly carried through the L-type calcium channel $\left(\mathrm{I}_{\mathrm{CaL}}\right.$ ) and (depending on the membrane potential and local sodium and calcium concentrations) on the sodium-calcium exchanger $\left(\mathrm{I}_{\mathrm{NaCa}}\right)$. It is the entry of calcium during this time frame that causes activation of the ryanodine receptors and release of calcium from the sarcoplasmic reticulum (SR). This process of calcium-induced calcium release is essential for excitation-contraction coupling and enables each action potential to be transduced into a mechanical event, allowing blood to be pumped around the body ${ }^{14}$.

Following on from the plateau phase, inactivation of the calcium channels combined with increases in the outward potassium current lead to terminal repolarization (phase 3). Finally, outward potassium and pump currents are responsible for the maintenance of the resting membrane potential (phase 4).

As it can be seen from Figure 4, there are species differences in the morphology of these action potentials. For example, $I_{K s}$ is much larger in guinea pigs when compared to canine or human cells and $\mathrm{I}_{\mathrm{CI}(\mathrm{Ca})}$ does not seem to play a role in phase-1 repolarization in human or guinea-pig cells. However, the general principles remain the same for the three species shown. This is not the case in rat and mouse, where the action potentials are of very short duration due to the fact that the major repolarizing current is $\mathrm{I}_{\mathrm{TO}}$. For this reason, it is not recommended that these species are used for the assessment of compounds on action-potential properties in safety-pharmacology studies. 
Canine
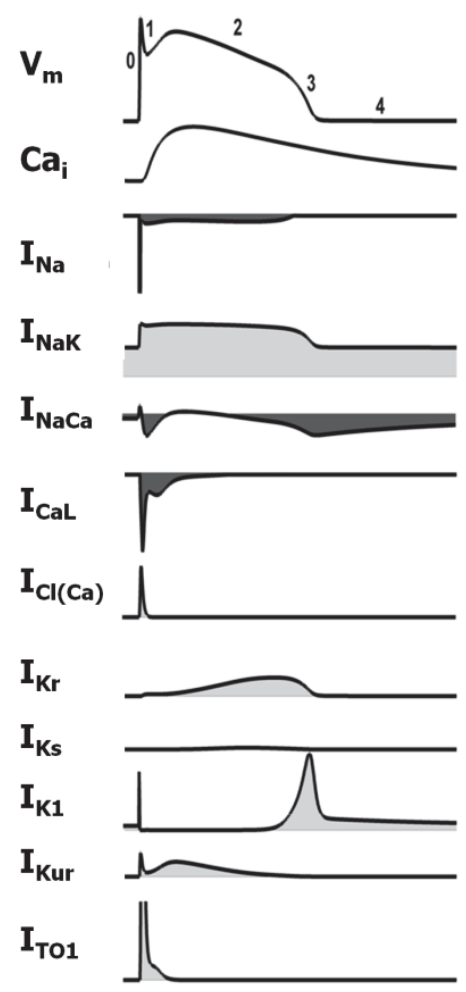

Human
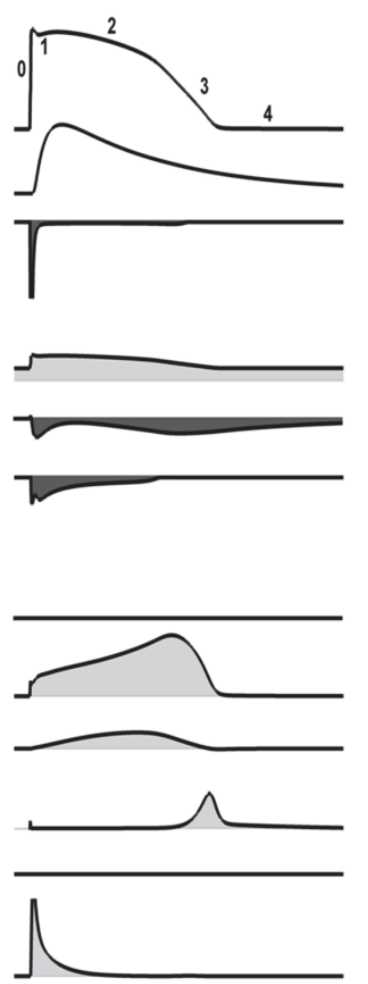

Guinea-Pig
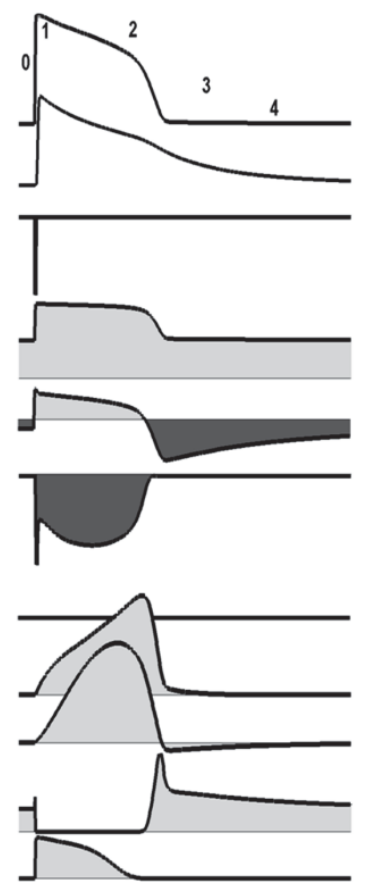

Figure 4: Representation of the inward and outward currents that contribute to the ventricular action potential in 3 different species. In addition, the cytoplasmic calcium transient is shown. Current amplitudes are relative to one another and were generated using 3 different computer models of the cardiac action potential ${ }^{15-17}$. Phases $0-4$ are noted for each action potential. (Courtesy of Jordi Heijman, PhD).

In addition to the species differences that are noted in Figure 4, there are also regional differences in the expression of the various ion channels throughout the heart, and this can contribute to different action-potential characteristics. In dog ${ }^{18}$, human ${ }^{19}$ and guinea-pig ${ }^{20}$, the action potential of epicardial myocytes is shorter in duration than that of endocardial cells. In addition, the spike-and-dome configuration is more prominent in the epicardium than the endocardium. There is also regional heterogeneity in calcium-handling proteins ${ }^{21}$ and recent work has demonstrated transmural heterogeneity of excitation-contraction coupling and calcium handling in human hearts ${ }^{22}$. Various studies ${ }^{23,}{ }^{24}$ have also illustrated that cells from the mid-myocardium have a longer action-potential duration than those from the endocardium and are more susceptible to prolongation, e.g., by blockade of $\mathrm{I}_{\mathrm{kr}}{ }^{25}$. However, the functional and clinical relevance of these ' $M$ cells' is still under debate ${ }^{26,27}$. Finally, there are also large interventricular differences in ion currents that contribute to dispersion of repolarization ${ }^{28}$. These regional electrical gradients caused by the differences in action-potential duration are responsible for the $T$ wave on the electrocardiogram ${ }^{29,}{ }^{30}$. Under various pathological conditions, exaggerated dispersion of repolarization creates the substrate for reentrant arrhythmias ${ }^{31}$. 
In 1998, the term "repolarization reserve" was introduced by Roden ${ }^{32}$. According to this concept, the inhibition or functional impairment of one transmembrane ion channel does not automatically result in excessive repolarization changes due to the fact that other currents that are normally redundant can compensate for the current that is reduced. This "reserve" can be augmented by various factors, including sympathetic tone, diet (e.g. grapefruit juice ${ }^{33}$ ) and disease (e.g. diabetes ${ }^{34}$; see recent review by Varro et al. ${ }^{35}$ ). In certain circumstances, however, the "reserve" is not sufficient to compensate for the challenge that is imposed upon it. Clinically, among conditions in which the repolarization reserve can be challenged are the congenital long-QT syndromes (LQTS), with a prevalence of around 1 in 2500 live births ${ }^{36}$. In long-QT patients the duration of repolarization, as measured on the ECG as the time interval between the beginning of the $Q$ wave and the end of the $T$ wave, is prolonged. The two most common LQT syndromes are caused by mutations in the KCNQ1 gene (encoding for the a-subunit of the $I_{\mathrm{Ks}}$ channel) or in hERG (encoding for the $\alpha$-subunit of the $\mathrm{I}_{\mathrm{Kr}}$ channel), although at the time of this writing there have been 13 LQT syndromes described ${ }^{37-39}$. Symptomatic patients with these syndromes can present with syncope or cardiac arrest, often as the result of TdP arrhythmia, and it is thought that the mechanisms of arrhythmogenesis in these patients are also operative in patients with drug-induced TdP.

Afterdepolarizations are among the mechanisms that are thought to be involved in arrhythmogenesis in both drug-induced TdP and congenital LQT (Figure 5). These oscillations can lead to either triggered activity ${ }^{40}$ and/or functional block which may encourage re-entry circuits. These phenomena can be detected from the single cell to the tissue and can even be observed in the intact heart when monophasic action potentials are recorded ${ }^{41}$. They are defined as depolarizations of the cardiac action potential that can occur in phase 2,3 or 4 of the action potential ${ }^{42}$. Normally if they occur in phase 4 they are called delayed afterdepolarizations (DADs) and if they occur earlier on the action potential then they are termed early afterdepolarizations (EADs). There is now a general consensus that DADs are a result of a transient inward current $\left(\mathrm{I}_{\mathrm{TI}}\right)$ activated by intracellular $\mathrm{Ca}^{2+43}$. This transient inward current is mainly due to activation of the electrogenic $\mathrm{Na}^{+}-\mathrm{Ca}^{2+}$ exchanger (NCX), with the $\mathrm{Ca}^{2+}$-activated $\mathrm{Cl}^{-}$current $\left(\mathrm{I}_{\mathrm{CI}(\mathrm{Ca})}\right)$ contributing in some species ${ }^{44,45}$. The mechanisms underlying EADs are much less clear cut however, and remain a topic of debate. Early evidence suggested EADs were caused as a result of reactivation of $\mathrm{I}_{\mathrm{CaL}}$ due to the prolonged plateau phase of the action potential ${ }^{46,47}$. However, there is other experimental evidence that suggests that EADs may also be caused as a result of $I_{T I}$ activation by intracellular $\mathrm{Ca}^{2+}$, especially under conditions of $\mathrm{Ca}^{2+}$ overload ${ }^{48-50}$. 


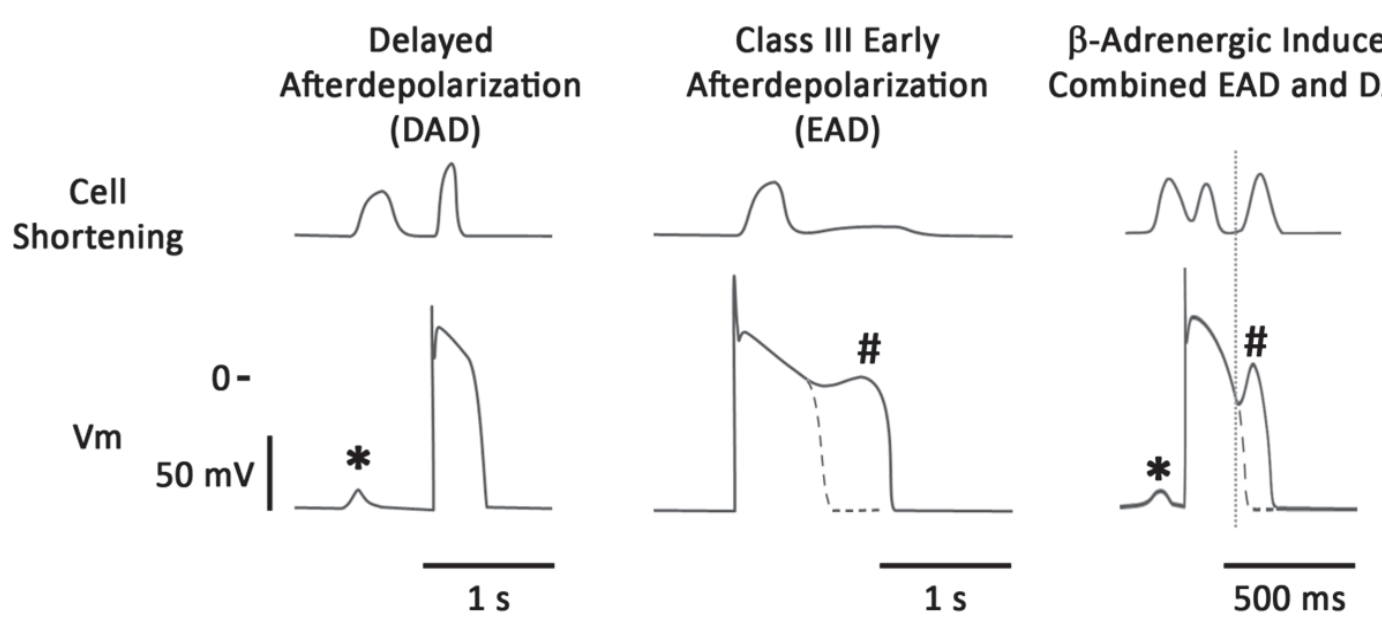

Figure 5: Representative examples of afterdepolarizations occurring in the single canine myocyte. Figure shows both membrane potentials and contraction for each situation. Left panel, DAD $\left(^{*}\right)$ induced by the $\beta$-adrenergic agonist isoproterenol (ISO); the middle panel illustrates an EAD (\#) induced by augmentation of the late sodium current, using ATX-II; the right panel illustrates that under certain conditions both types of afterdepolarizations can be seen in the same action potential. In this particular example, blockade of $I_{\mathrm{ks}}$ and $\beta$-adrenergic stimulation are the proarrhythmic treatment and it can be seen that an early aftercontraction initiates prior to the upstroke of the EAD.

\subsection{Proarrhythmic Models and Markers}

As TdP arrhythmias themselves are fortunately rare, strong surrogate markers are of crucial importance for the pharmaceutical industry for proarrhythmic risk assessment of novel chemical entities. In addition, various proarrhythmia models, both in-vivo and in-vitro, have been developed. These models share the characteristic of a reduced repolarization reserve, by different inciting mechanisms, which primes them for arrhythmia.

In clinical reality, many patients with TdP do not have a 'perfect' unremodeled heart, which illustrates the importance of checking for proarrhythmic activity in disease models. The dog with chronic complete atrioventricular block (CAVB) is one such in-vivo model. It has been used for over a century ${ }^{51}$ and since $1995{ }^{52}$ has received much attention as a model of TdP. After ablation of the atrioventricular node, electrical conduction from the atria to the ventricles is interrupted, resulting in a slower idioventricular rhythm to determine the heart rate. Due to this, cardiac output is initially reduced and over a period of 2-5 weeks ventricular hypertrophy occurs as a compensatory mechanism ${ }^{53}$. At the cellular level, the delayed rectifier $\mathrm{K}^{+}$current $\mathrm{I}_{\mathrm{Ks}}$ is reduced ${ }^{54}$, whereas sarcoplasmic reticulum $\mathrm{Ca}^{2+}$ release and $\mathrm{I}_{\mathrm{NaCa}}$ are enhanced ${ }^{55}$. Taken together, these alterations predispose to TdP arrhythmias, making the CAVB dog a sensitive model for TdP detection. Another in-vivo model used to detect the proarrhythmic potential of new compounds is the methoxamine- 
sensitized rabbit ${ }^{56}$. This model was first described in the early $1990 \mathrm{~s}^{57}$. Even today the exact mechanisms of $\alpha$-adrenoreceptor sensitization for TdP in this model are still unclear, however altered $\mathrm{Ca}^{2+}$ handling is likely to play a role.

Over recent years, various newer animal models have been described. $\beta$-adrenergic receptor ( $\beta A R$ ) stimulation with isoproterenol (ISO) reproducibly produced $\mathrm{TdP}$ in anesthetized dogs with a reduced repolarization reserve caused by selective $\mathrm{I}_{\mathrm{Ks}}$ blockade ${ }^{58,59}$. Interestingly under these conditions, aftercontractions were observed on the left-ventricular-pressure (LVP) signal prior to TdP induction, suggesting that abnormal $\mathrm{Ca}^{2+}$ handling plays a pivotal arrhythmogenic role. A combination of $\mathrm{I}_{\mathrm{Kr}}$ and $\mathrm{I}_{\mathrm{Ks}}$ blockade has also been shown to induce TdP in conscious dogs and anaesthetized rabbits ${ }^{60}$. Finally, in guinea pigs, adrenaline has been used to reveal the torsadogenic potential of a combination of $\mathrm{E}-4031$ ( $\mathrm{I}_{\mathrm{Kr}}$ blocker) and HMR1556 (I $\mathrm{I}_{\mathrm{Ks}}$ blocker) ${ }^{61}$.

In addition to the in-vivo models mentioned above, there are also a number of in-vitro models, as reviewed by Lawrence et al. ${ }^{62}$. Perhaps the most widely studied in-vitro model is the perfused and paced Langendorff-mounted rabbit heart. Many drugs with clinical proarrhythmic properties can be detected in this model ${ }^{9,63,64}$.

As already noted above, the most commonly used surrogate marker for TdP liability is the QT interval of the ECG. Although this biomarker has been adopted by regulatory agencies, in both preclinical and clinical studies, the link between a prolonged QT interval and arrhythmia is not watertight by any means. Numerous studies have indicated a discordance between QT prolongation and TdP induction. For example, Carlsson et al. ${ }^{65}$ showed that QT prolongation alone could not discriminate between methoxamine-sensitized rabbits that showed TdP and those that did not. In this particular study, the $\mathrm{I}_{\mathrm{Kr}}$ blocker almokalant was infused at different rates. In the animals subjected to a high rate of infusion of the compound, TdP was seen in 9 out of 10 rabbits and this was accompanied by an increase in QT of 30\%. Interestingly in the rabbits receiving a lower rate infusion, an increase in QT time of $42 \%$ was seen, however TdP was only seen in 1 out of 8 animals. In the clinical setting, in patients with acquired prolongation of the QT interval, Gilmour et al. (1997) ${ }^{66}$ showed that QT-interval prolongation alone was insufficient to account for the initiation of TdP, suggesting that other arrhythmogenic factors are more relevant.

Over recent years, more direct markers of TdP prediction have been proposed. Among them are: TRIaD, beat-to-beat variability of repolarization (BVR) and the electromechanical window (EMW). 
In a study of Hondeghem et al. of $2001^{9}$, the investigators characterized 702 chemical entities in Langendorff-perfused rabbit hearts, and then measured various determinants, collectively known as TRIad. Triangulation (' $\left.\mathrm{T}^{\prime}\right)$ of the action potential (taken as the repolarization time from APD30 to APD90), reverse-use dependence $\left({ }^{\prime}\right.$ '), temporal instability of the action potential duration ( $\left.\mathrm{I}^{\prime}\right)$, and dispersion of repolarization (spatial and temporal, ' $D$ ') were all measured. In these experiments, the instability was quantified by means of a so-called "instability index". This index was computed as the difference between the upper and lower quartiles of APD60 during the last 60 beats during drug perfusion. However, this parameter does not take into account the consecutiveness of the beat-to-beat repolarization instability. Hondeghem and his colleagues then went on to plot the instability index against APD prolongation. In this setting, a number of chemical entities induced considerable repolarization instability while producing only minimal lengthening of the APD. TdPlike polymorphic ventricular tachyarrhythmias were often seen under these conditions. Vice versa, there were also compounds that lengthened the APD considerably but left temporal instability minimally altered. These compounds showed no or hardly any arrhythmogenic activity. In addition to TRIaD, more recently Hondeghem also included calculations of cardiac wavelength $(\lambda)$ as an additional risk marker ${ }^{67}$. Cardiac wavelength is the product of the effective refractory period and the conduction velocity and therefore takes into account additional factors that appear to influence arrhythmogenic outcome but otherwise would not be considered for arrhythmia risk. These data further question the utility of QT times alone as a proarrhythmic marker.

Beat-to-beat variability of repolarization (BVR) takes into account the consecutiveness of variability. As dispersion of repolarization seems to play an important role in arrhythmogenesis, it is likely that the consecutiveness of repolarization duration is also vital. For example, if the repolarization duration increases by the same increment throughout the heart then it is unlikely that arrhythmia will ensue, as there is only a minimal risk of functional conduction block. In 2004, Thomsen et al. ${ }^{10}$ quantified BVR as short-term variability (STV). This parameter was quantified as being the mean orthogonal distance from the line of identity to the most distant points of the Poincaré plot. These data can be derived by using the mathematical formula STV $=\Sigma\left|D_{n+1}-D_{n}\right| /\left[n_{\text {beats }} * \sqrt{ } 2\right]$, where $D$ represents the duration of repolarization. This marker has been most extensively studied in the CAVB dog where $D$ is derived from endocardial monophasic-action-potential recordings. In this model, multiple compounds have been tested and BVR has been assessed ${ }^{53}$. Interestingly, whereas BVR increases by the administration of many proarrhythmic agents, it can also be reduced when an anti-arrhythmic agent is applied ${ }^{68,69}$. Since its inception, the concept of BVR has been applied in multiple animal models ${ }^{58,70}$ and also to the clinical situation ${ }^{71-74}$ and, in the majority of conditions, it does seem to be a more reliable marker of arrhythmogenic risk compared to QT prolongation alone. 
Finally, the latest surrogate marker to be described for TdP risk is the EMW, which was recently characterized in dogs by van der Linde et al. ${ }^{59}$. Under normal conditions, the QT interval is shorter than the duration of the LVP (QLVP end), providing a positive EMW. Interestingly, atrial pacing, atropine or body temperature changes had no major effects on the EMW, whereas changes in the QT duration were considerable. However, during $\mathrm{I}_{\mathrm{Ks}}$ blockade in dogs, $\beta$ AR stimulation with ISO led to a large negative EMW and this was very often accompanied by TdP. Prevention of TdP by atenolol or verapamil was associated with a (much) lessnegative EMW. On the other hand mexiletine, a class-1B antiarrhythmic drug sometimes used to treat LQT3 patients, did not affect the EMW or prevent TdP induction. These initial data indicate that mismatches between the electrical and mechanical activity of the heart may be better indicators of arrhythmogenesis than electrical activity alone.

\subsection{Aims and Outline of this Thesis}

Based on the above, a greater understanding of the underlying mechanisms behind drug-induced TdP is required, not in the least to aid the pharmaceutical industry to develop safer compounds and to avoid that efficacious compounds are discarded on false assumptions. Hence, the main objective of the work presented in this thesis was to study the mechanisms involved in TdP arrhythmogenesis, focusing on the cellular aspects that underlie BVR, one of the newer surrogate markers thought to have utility in predicting this arrhythmia. Particular attention was paid to how BVR is altered under various proarrhythmic conditions and how this can contribute to arrhythmia formation.

In Chapter 2, we describe a protocol for isolating canine cardiac myocytes and then assessing changes in AP duration in canine ventricular myocytes utilizing optical imaging techniques. This technique is then validated by using various test compounds. In addition, a protocol is described that allows for the assessment of BVR in single canine myocytes. The protocols described in Chapter $\mathbf{2}$ were then applied in the studies underlying Chapters $\mathbf{3}$ and 4. In Chapter 3, isolated myocytes were used to investigate the importance of $\beta A R$ stimulation in the rescue of excessive increases in BVR following $I_{\mathrm{Kr}}$ blockade and augmented late $\mathrm{I}_{\mathrm{Na}}$. In addition, the effects of $\mathrm{I}_{\mathrm{Ks}}$ inhibition on BVR were investigated in the presence and absence of $\beta A R$ stimulation. These studies were important in defining the influence that alterations in the repolarization reserve could have on BVR and enable us to begin to delineate ionic mechanisms of BVR. In Chapter 4 additional cellular mechanisms of BVR are described. Here, we investigated the relationship between spontaneous $\mathrm{Ca}^{2+}$ releases and BVR using a combined experimental and computational approach under conditions of $\beta A R$ stimulation, with and without $\mathrm{I}_{\mathrm{Ks}}$ blockade. In addition a mechanism for this relationship is proposed. This chapter 
delves further into the ionic mechanisms of BVR and provides important insights into mechanisms behind TdP arrhythmias as well as aiding in the development of antiarrhythmic agents. Chapter 5 investigates interventricular differences in the regulation of cyclic AMP levels by phosphodiasterases (PDE), and how this can have differential effects on the ion channels that are regulated under this pathway, which may ultimately affect dispersion of repolarization and have arrhythmogenic consequences. In Chapter $\mathbf{6}$ we move from the single cell to the whole animal. In this study, the effect of a novel combined ion-channel blocker, AZD1305, was assessed for proarrhythmic liability in the CAVB dog, where repolarization reserve is already challenged by the remodeling process making these animals much more susceptible to TdP arrhythmia. In addition, BVR was monitored throughout these studies to further establish this parameter as a superior surrogate marker for TdP induction. In the studies for Chapter 7, we investigated the utility of the EMW in the anesthetized guinea-pig model after challenging these animals with known torsadogenic reference compounds. Finally, in Chapter $\mathbf{8}$ the findings of all chapters are discussed in an integrated manner and ideas for further research are outlined. 


\section{References}

(1) Kerr WJ BW. Paroxysmal ventricular fibrillation with cardiac recovery in a case of auricular fibrillation and complete heart block while under quinidine sulfate therapy. Heart. 1922;9:269-278.

(2) June RA, Nasr I. Torsades de pointes with terfenadine ingestion. Am J Emerg Med. 1997;15:542-3.

(3) Lu HR, Hermans AN, Gallacher DJ. Does terfenadine-induced ventricular etc Tachycardia/Fibrillation Directly Relate to Its QT Prolongation and Torsades de Pointes? Br J Pharmacol . 2012;166:1490-502.

(4) Shah RR. Drug-Induced QT Interval Prolongation: Does ethnicity of the thorough QT study population matter? Br J Clin Pharmacol. 2012 Aug 7.

(5) Kola I, Landis J. Can the pharmaceutical industry reduce attrition rates? Nat Rev Drug Discov. 2004;3:711-5.

(6) Laverty H, Benson C, Cartwright E, Cross M, Garland C, Hammond T, Holloway C, McMahon N, Milligan J, Park B, Pirmohamed M, Pollard C, Radford J, Roome N, Sager P, Singh S, Suter T, Suter W, Trafford A, Volders $P$, Wallis $R$, Weaver $R$, York $M$, Valentin J. How can we improve our understanding of cardiovascular safety liabilities to develop safer medicines? Br J Pharmacol. 2011;163:675-93.

(7) Shah RR. Can pharmacogenetics help rescue drugs withdrawn from the market? Pharmacogenomics. 2006;7:889-908.

(8) Redfern WS, Ewart L, Hammond TG, Bialeck R, Kinter L, Lindgren S, Pollard $C E$, Roberts $R$, Rolf MG, Valentin JP. Impact and frequency of different toxicities throughout the pharmaceutical life cycle. The Toxicologist. 2010;114(S1): 1081.

(9) Hondeghem LM, Carlsson L, Duker G. Instability and triangulation of the action potential predict serious proarrhythmia, but action potential duration prolongation is antiarrhythmic. Circulation. 2001;103:2004-13.

(10) Thomsen MB, Verduyn SC, Stengl M, Beekman JD, de Pater G, van Opstal J, Volders PG, Vos MA. Increased short-term variability of repolarization predicts d-sotalol-induced torsades de pointes in dogs. Circulation. 2004;110:2453-9. 
(11) Towart R, Linders JT, Hermans AN, Rohrbacher J, van der Linde HJ, Ercken M, Cik M, Roevens P, Teisman A, Gallacher DJ. Blockade of the $I_{K s}$ potassium channel: an overlooked cardiovascular liability in drug safety screening? J Pharmacol Toxicol Methods. 2009;60:1-10.

(12) Lacerda AE, Kuryshev YA, Chen Y, Renganathan M, Eng H, Danthi SJ, Kramer JW, Yang T, Brown AM. Alfuzosin delays cardiac repolarization by a novel mechanism. J Pharmacol Exp Ther. 2008;324:427-33.

(13) Draper $\mathrm{MH}$, Weidmann S. Cardiac resting and action potentials recorded with an intracellular electrode. J Physiol .1951;115:74-94.

(14) Bers DM. Cardiac excitation-contraction coupling. Nature 2002 January 10;415:198-205.

(15) Heijman J, Volders PG, Westra RL, Rudy Y. Local control of $\beta$-adrenergic stimulation: Effects on ventricular myocyte electrophysiology and $\mathrm{Ca}^{2+}$ transient. J Mol Cell Cardiol. 2011;50:863-71.

(16) O'Hara T, Virág L, Varró A, Rudy Y. Simulation of the undiseased human cardiac ventricular action potential: model formulation and experimental validation. PLoS Comput Biol. 2011;7:e1002061.

(17) Faber GM, Rudy Y. Action potential and contractility changes in $\left[\mathrm{Na}^{+}\right]_{\mathrm{i}}$ overloaded cardiac myocytes: a simulation study. Biophys J. 2000;78:2392-404.

(18) Litovsky SH, Antzelevitch C. Transient outward current prominent in canine ventricular epicardium but not endocardium. Circ Res. 1988;62:116-26.

(19) Nabauer M, Beuckelmann DJ, Uberfuhr P, Steinbeck G. Regional differences in current density and rate-dependent properties of the transient outward current in subepicardial and subendocardial myocytes of human left ventricle. Circulation. 1996;93:168-77.

(20) Bryant SM, Wan X, Shipsey SJ, Hart G. Regional differences in the delayed rectifier current $\left(\mathrm{I}_{\mathrm{Kr}}\right.$ and $\mathrm{I}_{\mathrm{Ks}}$ ) contribute to the differences in action potential duration in basal left ventricular myocytes in guinea-pig. Cardiovasc Res. 1998;40:322-31.

(21) Laurita KR, Katra R, Wible B, Wan X, Koo MH. Transmural heterogeneity of calcium handling in canine. Circ Res. 2003;92:668-75.

(22) Lou Q, Fedorov VV, Glukhov AV, Moazami N, Fast VG, Efimov IR. Transmural heterogeneity and remodeling of ventricular excitation-contraction coupling in human heart failure. Circulation. 2011;123:1881-90. 
(23) Sicouri S, Antzelevitch C. A subpopulation of cells with unique electrophysiological properties in the deep subepicardium of the canine ventricle. The M cell. Circ Res. 1991;68:1729-41.

(24) Anyukhovsky EP, Sosunov EA, Rosen MR. Regional differences in electrophysiological properties of epicardium, midmyocardium, and endocardium. In vitro and in vivo correlations. Circulation. 1996;94:1981-8.

(25) Liu DW, Antzelevitch C. Characteristics of the delayed rectifier current ( $\mathrm{I}_{\mathrm{kr}}$ and $\mathrm{I}_{\mathrm{Ks}}$ ) in canine ventricular epicardial, midmyocardial, and endocardial myocytes. A weaker $I_{\mathrm{Ks}}$ contributes to the longer action potential of the M cell. Circ Res. 1995;76:351-65.

(26) Wilson LD, Jennings MM, Rosenbaum DS. Point: $M$ cells are present in the ventricular myocardium. Heart Rhythm. 2011;8:930-3.

(27) Janse MJ, Coronel R, Opthof T. Counterpoint: M cells do not have a functional role in the ventricular myocardium of the intact heart. Heart Rhythm. 2011;8:934-7.

(28) Volders PG, Sipido KR, Carmeliet E, Spätjens RL, Wellens HJ, Vos MA. Repolarizing $\mathrm{K}^{+}$currents $\mathrm{I}_{\mathrm{TO} 1}$ and $\mathrm{I}_{\mathrm{Ks}}$ are larger in right than left canine ventricular midmyocardium. Circulation. 1999;99:206-10.

(29) Burgess MJ. Relation of ventricular repolarization to electrocardiographic T wave-form and arrhythmia vulnerability. Am J Physiol. 1979;236:H391-H402.

(30) Opthof T, Coronel R, Wilms-Schopman FJ, Plotnikov AN, Shlapakova IN, Danilo $\mathrm{P}$ Jr, Rosen MR, Janse MJ. Dispersion of repolarization in canine ventricle and the electrocardiographic $T$ wave: Tp-e interval does not reflect transmural dispersion. Heart Rhythm. 2007;4:341-8.

(31) Antzelevitch C, Fish J. Electrical heterogeneity within the ventricular wall. Basic Res Cardiol .2001;96:517-27.

(32) Roden DM. Taking the "idio" out of "idiosyncratic": predicting torsades de pointes. Pacing Clin Electrophysiol. 1998;21:1029-34.

(33) Zitron E, Scholz E, Owen RW, Lück S, Kiesecker C, Thomas D, Kathöfer S, Niroomand F, Kiehn J, Kreye VA, Katus HA, Schoels W, Karle CA. QTC prolongation by grapefruit juice and its potential pharmacological basis: HERG channel blockade by flavonoids. Circulation. 2005;111:835-8. 
(34) Lengyel C, Virág L, Bíró T, Jost N, Magyar J, Biliczki P, Kocsis E, Skoumal R, Nánási PP, Tóth $M$, Kecskeméti $V$, Papp JG, Varró A. Diabetes mellitus attenuates the repolarization reserve in mammalian heart. Cardiovasc Res. 2007;73:512-20.

(35) Varro A, Baczko I. Cardiac ventricular repolarization reserve: a principle for understanding drug-related proarrhythmic risk. $\mathrm{Br} J$ Pharmacol. 2011;164:14-36.

(36) Crotti L, Celano G, Dagradi F, Schwartz PJ. Congenital long QT syndrome. Orphanet J Rare Dis. 2008;3:18.

(37) El-Sherif N, Turitto G. The long QT syndrome and torsade de pointes. Pacing Clin Electrophysiol . 1999;22:91-110.

(38) Goldenberg I, Moss AJ. Long QT syndrome. J Am Coll Cardiol. 2008;51:2291-300.

(39) Cerrone M, Priori SG. Genetics of sudden death: focus on inherited channelopathies. Eur Heart J. 2011;32:2109-18.

(40) Wit AL, Rosen MR. Pathophysiologic mechanisms of cardiac arrhythmias. Am Heart J. 1983;106:798-811.

(41) Priori SG, Mantica M, Napolitano C, Schwartz PJ. Early afterdepolarizations induced in vivo by reperfusion of ischemic myocardium. A possible mechanism for reperfusion arrhythmias. Circulation. 1990;81:1911-20.

(42) Cranefield PF. Action potentials, afterpotentials, and arrhythmias. Circ Res. 1977;41:415-23.

(43) Marban E, Robinson SW, Wier WG. Mechanisms of arrhythmogenic delayed and early afterdepolarizations in ferret ventricular muscle. $J$ Clin Invest. 1986;78:1185-92.

(44) Fedida D, Noble D, Rankin AC, Spindler AJ. The arrhythmogenic transient inward current $\dot{i}_{\text {II }}$ and related contraction in isolated guinea-pig ventricular myocytes. J Physiol. 1987;392:523-42.

(45) Zygmunt AC, Goodrow RJ, Weigel CM. $I_{\mathrm{NaCa}}$ and $I_{\mathrm{CI}(\mathrm{Ca})}$ contribute to isoproterenol-induced delayed after depolarizations in midmyocardial cells. Am J Physiol. 1998;275:H1979-H1992.

(46) January CT, Riddle JM. Early afterdepolarizations: mechanism of induction and block. A role for L-type $\mathrm{Ca}^{2+}$ current. Circ Res. 1989;64:977-90. 
(47) Zeng J, Rudy Y. Early afterdepolarizations in cardiac myocytes: mechanism and rate dependence. Biophys J. 1995;68:949-64.

(48) Priori SG, Corr PB. Mechanisms underlying early and delayed afterdepolarizations induced by catecholamines. $A m \mathrm{~J}$ Physiol. 1990;258:H1796-H1805.

(49) Volders PG, Kulcsar A, Vos MA, Sipido KR, Wellens HJ, Lazzara R, Szabo B. Similarities between early and delayed afterdepolarizations induced by isoproterenol in canine ventricular myocytes. Cardiovasc Res. 1997;34:348-59.

(50) Volders PG, Vos MA, Szabo B, Sipido KR, De Groot SH, Gorgels AP, Wellens $\mathrm{HJ}$, Lazzara R. Progress in the understanding of cardiac early afterdepolarizations and torsades de pointes: time to revise current concepts. Cardiovasc Res. 2000;46:376-92.

(51) Erlanger J, Blackman JR. Further studies in the physiology of the heart block in mammals. Chronic auriculo-ventricular heart block in the dog. Heart. 1910;1:177.

(52) Vos MA, Verduyn SC, Gorgels AP, Lipcsei GC, Wellens HJ. Reproducible induction of early afterdepolarizations and torsade de pointes arrhythmias by d-sotalol and pacing in dogs with chronic atrioventricular block. Circulation. 1995;91:864-72.

(53) Oros A, Beekman JD, Vos MA. The canine model with chronic, complete atrio-ventricular block. Pharmacol Ther. 2008;119:168-78.

(54) Volders PG, Sipido KR, Vos MA, Spätjens RL, Leunissen JD, Carmeliet E, Wellens $\mathrm{HJ}$. Downregulation of delayed rectifier $\mathrm{K}^{+}$currents in dogs with chronic complete atrioventricular block and acquired torsades de pointes. Circulation. 1999;100:2455-61.

(55) Sipido KR, Volders PG, De Groot SH, Verdonck F, Van de Werf F, Wellens HJ, Vos MA. Enhanced $\mathrm{Ca}^{2+}$ release and $\mathrm{Na} / \mathrm{Ca}$ exchange activity in hypertrophied canine ventricular myocytes: potential link between contractile adaptation and arrhythmogenesis. Circulation. 2000;102(17): 2137-44.

(56) Carlsson L. The anaesthetised methoxamine-sensitised rabbit model of torsades de pointes. Pharmacol Ther. 2008;119:160-7.

(57) Carlsson L, Almgren O, Duker G. QTU-prolongation and torsades de pointes induced by putative class III antiarrhythmic agents in the rabbit: etiology and interventions. J Cardiovasc Pharmacol.1999;16:276-85. 
(58) Gallacher DJ, Van de Water A, van der Linde H, Hermans AN, Lu HR, Towart $\mathrm{R}$, Volders PG. In vivo mechanisms precipitating torsades de pointes in a canine model of drug-induced long-QT1 syndrome. Cardiovasc Res. 2007;76:247-56.

(59) van der Linde HJ, Van Deuren B, Somers Y, Loenders B, Towart R, Gallacher DJ. The Electro-Mechanical window: a risk marker for Torsade de Pointes in a canine model of drug induced arrhythmias. $\mathrm{Br} J$ Pharmacol. 2010;161:1444-54.

(60) Lengyel C, Varro A, Tabori K, Papp JG, Baczko I. Combined pharmacological block of $I_{\mathrm{Kr}}$ and $I_{\mathrm{Ks}}$ increases short-term QT interval variability and provokes torsades de pointes. Br J Pharmacol. 2007;151:941-51.

(61) Michael G, Kane KA, Coker SJ. Adrenaline reveals the torsadogenic effect of combined blockade of potassium channels in anaesthetized guinea pigs. Br J Pharmacol. 2008;154:1414-26.

(62) Lawrence $\mathrm{CL}$, Pollard $\mathrm{CE}$, Hammond TG, Valentin JP. In vitro models of proarrhythmia. Br J Pharmacol. 2008;154:1516-22.

(63) Hondeghem LM, Hoffmann P. Blinded test in isolated female rabbit heart reliably identifies action potential duration prolongation and proarrhythmic drugs: importance of triangulation, reverse use dependence, and instability. J Cardiovasc Pharmacol. 2003;41:14-24.

(64) Hondeghem LM, Lu HR, van RK, De CF. Detection of proarrhythmia in the female rabbit heart: blinded validation. J Cardiovasc Electrophysiol. 2003;14:287-94.

(65) Carlsson L, Abrahamsson C, Andersson B, Duker G, Schiller-Linhardt G. Proarrhythmic effects of the class III agent almokalant: importance of infusion rate, QT dispersion, and early afterdepolarisations. Cardiovasc Res. $1993 ; 27: 2186-93$.

(66) Gilmour RF, Jr., Riccio ML, Locati EH, Maison-Blanche P, Coumel P, Schwartz $\mathrm{PJ}$. Time- and rate-dependent alterations of the QT interval precede the onset of torsade de pointes in patients with acquired QT prolongation. J Am Coll Cardiol. 1997;30:209-17.

(67) Hondeghem LM, Dumotier B, Traebert M. Oscillations of cardiac wave length and proarrhythmia. Naunyn Schmiedebergs Arch Pharmacol. 2010;382:367-76. 
(68) Oros A, Houtman MJ, Neco P, Gomez AM, Rajamani S, Oosterhoff P, Attevelt NJ, Beekman JD, van der Heyden MA, Ver DL, Belardinelli L, Richard S, Antoons G, Vos MA. Robust anti-arrhythmic efficacy of verapamil and flunarizine against dofetilide-induced TdP arrhythmias is based upon a shared and a different mode of action. Br J Pharmacol. 2010;161:162-75.

(69) Antoons G, Oros A, Beekman JD, Engelen MA, Houtman MJ, Belardinelli L, Stengl M, Vos MA. Late $\mathrm{Na}^{+}$current inhibition by ranolazine reduces torsades de pointes in the chronic atrioventricular block dog model. $J$ Am Coll Cardiol. 2010;55:801-9.

(70) Jacobson I, Carlsson L, Duker G. Beat-by-beat QT interval variability, but not QT prolongation per se, predicts drug-induced torsades de pointes in the anaesthetised methoxamine-sensitized rabbit. J Pharmacol Toxicol Methods. 2011;63:40-6.

(71) Hinterseer M, Beckmann BM, Thomsen MB, Pfeufer A, Dalla PR, Loeff M, Netz $H$, Steinbeck G, Vos MA, Kääb S. Relation of increased short-term variability of QT interval to congenital long-QT syndrome. Am J Cardiol. 2009;103:1244-8.

(72) Hinterseer M, Thomsen MB, Beckmann BM, Pfeufer A, Schimpf R, Wichmann $\mathrm{HE}$, Steinbeck G, Vos MA, Kääb S. Beat-to-beat variability of QT intervals is increased in patients with drug-induced long-QT syndrome: a case control pilot study. Eur Heart J. 2008;29:185-90.

(73) Hinterseer M, Beckmann BM, Thomsen MB, Pfeufer A, Ulbrich M, Sinner MF, Perz S, Wichmann HE, Lengyel C, Schimpf R, Maier SK, Varro A, Vos MA, Steinbeck G, Kääb S. Usefulness of short-term variability of QT intervals as a predictor for electrical remodeling and proarrhythmia in patients with nonischemic heart failure. Am J Cardiol. 2010;106:216-20.

(74) Lengyel C, Orosz A, Hegyi P, Komka Z, Udvardy A, Bosnyak E, Trajer E, Pavlik G, Toth M, Wittmann T, Papp JG, Varro A, Baczko I. Increased shortterm variability of the QT interval in professional soccer players: possible implications for arrhythmia prediction. PLoS One. 2011;6:e18751. 


\section{Chapter 2}

\section{Measurement of Action Potential Generation in I solated Canine Left Ventricular Midmyocardial Myocytes}

Curr Protoc Pharmacol. 2011;Chapter 10:Unit 10.14.1-23

Daniel M Johnson • Leyla Hussein • Roel LHMG Spätjens • Jean-Pierre Valentin •

Paul GA Volders Najah Abi-Gerges 


\section{Abstract}

Proarrhythmic side effects are a major limitation during the drug development process for cardiac and non-cardiac compounds. Because changes in cardiac action potential (AP) are undesirable, the evaluation of the effects of test compounds on the AP is essential before advancing new compounds to clinical testing. However, an increase in repolarization duration alone is not always proarrhythmic, and newer surrogate markers have been suggested to better predict the occurrence of arrhythmia. Described in this chapter is a protocol for assessing changes in AP duration in canine ventricular myocytes utilizing optical imaging techniques. This protocol can be used at an early stage of drug discovery due to its relatively fast throughput. Additionally, a protocol is presented for assessing the occurrence of after-depolarizations, as well as a novel parameter for proarrhythmic risk, beat-tobeat variability of repolarization. This protocol can be used at a later stage of the drug discovery process to assess proarrhythmic potential. 


\subsection{I ntroduction}

Regulatory guidance documents ${ }^{1,2}$ rely on prolongation of the QT interval as a biomarker for predicting the risk of compound-induced Torsades de Pointes (TdP). As changes in cardiac action potential (AP) are undesirable, the evaluation of the effects of test compounds on the AP is essential before advancing new chemical entities (NCEs) to human testing. Compound throughput in the single myocyte assay is much greater than with conventional APD models, and animal use is reduced. However, the technical difficulties associated with making sharp-electrode (SE) recordings in these cells limit the number of test compounds that can be assessed. Hence, cell lines expressing key cardiac ion channels (IC), and the advent of automated electrophysiology, have made it possible to "molecularise" the AP as a means of early QT risk assessment. Nevertheless, no matter how comprehensive the panel of ICs becomes, there is always the need to assess test compounds in an integrated system (e.g., myocyte, whole animal). Accordingly, a new method employing a fast voltagesensitive dye (e.g., di-4-ANEPPS) to record APs in left ventricular midmyocardial myocytes (LVMMs) and which is technically less challenging has been developed in our laboratories. Because of these attributes, it provides an experimental AP duration (APD) assay with high throughput, making it possible to more quickly identify and discard at an earlier stage of the drug discovery process NCEs that may potentially modify APD ${ }^{3}$.

A prolonged QT interval cannot reliably predict the pro-arrhythmic potential of a test compound ${ }^{4,5}$. As a result, rather than simply assessing the QT interval prolongation risk, efforts are being made to identify more reliable preclinical proarrhythmic markers that correlate with the incidence of $\mathrm{TdP}$, such as beat-to-beat variability of repolarization (BVR), which is quantified as short-term variability (STV(APD)) in LVMM cells ${ }^{4,6,7}$. Because one of the limitations to optical measurements from LVMM cells using di-4-ANEPPS is the need to limit measurements to finite time periods to avoid phototoxic effects of the dye, measurement of the putative proarrhythmic markers STV(APD), early after-depolarizations (EADs) and delayed afterdepolarizations (DADs), could only occur using SE methodology. Therefore, the latter methodology can be used to assess the proarrhythmic potential of a test compound in LVMM cells at a late phase of the drug discovery process.

Detailed below is a method for studying APs in isolated canine LVMM cells. The focus is measurement of changes in the AP parameters induced by superfusion with a test compound (Basic Protocol) using di-4-ANEPPS. In addition, a protocol is described for investigating the arrhythmogenic effects of a test compound on temporal STV(APD) and the occurrence of EADs and DADs using the SE technique (Alternate Protocol 1). As $\beta$-adrenergic receptor ( $\beta A R$ ) stimulation is an important contributor to arrhythmogenesis under conditions where repolarization is impaired by a reduction in the slow activated potassium current $\left(\mathrm{I}_{\mathrm{Ks}}\right)$, as occurs in association 
with either inherited or acquired long QT1 syndrome ${ }^{7,8}$, a second alternate protocol is included that makes possible an investigation of the effects of a test compound in combination with isoproterenol (ISO), a $\beta A R$ agonist.

NOTE: All protocols using live animals must first be reviewed and approved by an Institutional Animal Care and Use Committee (IACUC) or must conform to governmental regulations regarding the care and use of laboratory animals.

\subsection{Basic Protocol: Measurement of Test Compound-Induced Changes in Action Potential Parameters in the Dog Midmyocardial Myocytes}

Described in this protocol is a procedure for recording APs in isolated dog LVMMs and measuring changes in AP parameters induced by a test compound. Measurement of the AP is accomplished using di-4-ANEPPS, a voltage-sensitive dye.

\subsubsection{Materials}

- Female beagle dog ( $\geq 9 \mathrm{~kg}, 9-35$ months)

- Normal Tyrode's solution (see recipe)

- Calcium-free and containing 0.2 and $1.8 \mathrm{mM} \mathrm{CaCl}_{2}$

- Bovine albumin serum (BSA, Sigma)

- Pentobarbitone

- $1 \mathrm{M} \mathrm{CaCl}_{2}$

- Collagenase A (Roche Diagnostics)

- $70 \%$ and $100 \%(\mathrm{v} / \mathrm{v}$ ) ethanol (in water)

- Di-4-ANEPPS (Sigma)

- Test compound

- Reference compound (e.g., dofetilide, a class III antiarrhythmic agent)

- Dimethyl sulfoxide (DMSO; Sigma)

Equipment and supplies for myocyte isolation (Figure 1, left panel) plus: Microosmometer (e.g., Advanced Instruments model 3300)

- $\quad$ H meter (e.g., SevenMulti, Mettler Toledo)

- 1-liter sterile Schott Duran bottles (LabPlanet, IL, USA)

- Gassing system to deliver medical $\mathrm{O}_{2}$ (99.5\%, type $\mathrm{F}$ cylinder)

- Oxygenating bubbler

- Surgical scissors, straight, $S / S, 105$ mm (4-1/8 in.)

- Mayo dissection scissors, straight, $B / B, 140 \mathrm{~mm}(5-1 / 2 \mathrm{in}$.)

- Medium dissecting forceps, straight, $160 \mathrm{~mm}$ (6.30 in.)

- Aesculap Iris dissection forceps (Ted Pella)

- 100-, 200-, and 500-ml Beakers

- Sewing needle and thread 
- Waste tissue bag

- 50-ml BD Plastika syringe and Polyethylene tubing

- Heating coil (Radnoti)

- DC50-B5 heating circulator (HAAKE, Thermo Fisher Scientific)

- Perfusion system

- (R3603, Tygon, Saint-Gobain Performance Plastics Corporation)

- Cannula (vessel dilator $1.65 \times 0.53$ mm; Cordis, J\&J Company)

- 10-ml Syringes

- 130-mm Dissection dishes without rubber silicone base

- Cell dissociation sieve tissue grinder kit (Sigma) with Screens for CD-1(100- $\mu \mathrm{m}$ mesh)

- Inverted microscope (e.g., Nikon Eclipse TS100)

Equipment and supplies for recording optical AP (Figure 2), including:

- Antivibration table (Scientifica) and Faraday cage (Type II, Scientifica), covered with materials that prevent entry of light

- Inverted microscope (e.g., Nikon Eclipse TE200) with 40x oil objective (1.30 NA, Nikon)

- FHD microscope chamber system (IonOptix)

- Cell MicroControls mTCII temperature controller and heater (IonOptix, Dublin, Ireland)

- CF-8vs valve assembly and cFlow controller (IonOptix)

- Immersion oil for fluorescence and general microscopy (Cargille Laboratories)

- Long-pass (>700 nm) and narrow band-pass (540-580 and 600-640 nm) filters

- Chromatic reflectors ( $<590$ and $<700 \mathrm{~nm}$ )

- Dual-emission photometry system with two photomultiplier tubes (PMTs), each with an amplifier and high-voltage power supply (Cairn Research)

- Xenon arc lamp power supply associated with arc lamp + housing (Cairn Research)

- Cairn shutter supply associated with xenon arc lamp + housing (Cairn Research)

- TH-10Kmp-thermistor probe (Cell MicroControls)

- Bath-mounted platinum wire electrodes

- Infrared camera (Watec) and Vista 14-inch monitor (Norbain SD)

- HSE stimulator P (Hugo Sachs Elektronik) or MyoPacer Field Stimulator (IonOptix)

- MultiClamp 700A amplifier and Digidata 1322A digitizer (Molecular Devices)

- Computer with pClamp 10 software (Molecular Devices) for acquisition and analysis of electrical signal

- Corning cover glass (no. 1, 25-mm sq; Corning Life Sciences)

- Vacuum pump (Rena Air 200, Planet Rena)

- Lens cleaning tissue (Thermo Fisher Scientific) 

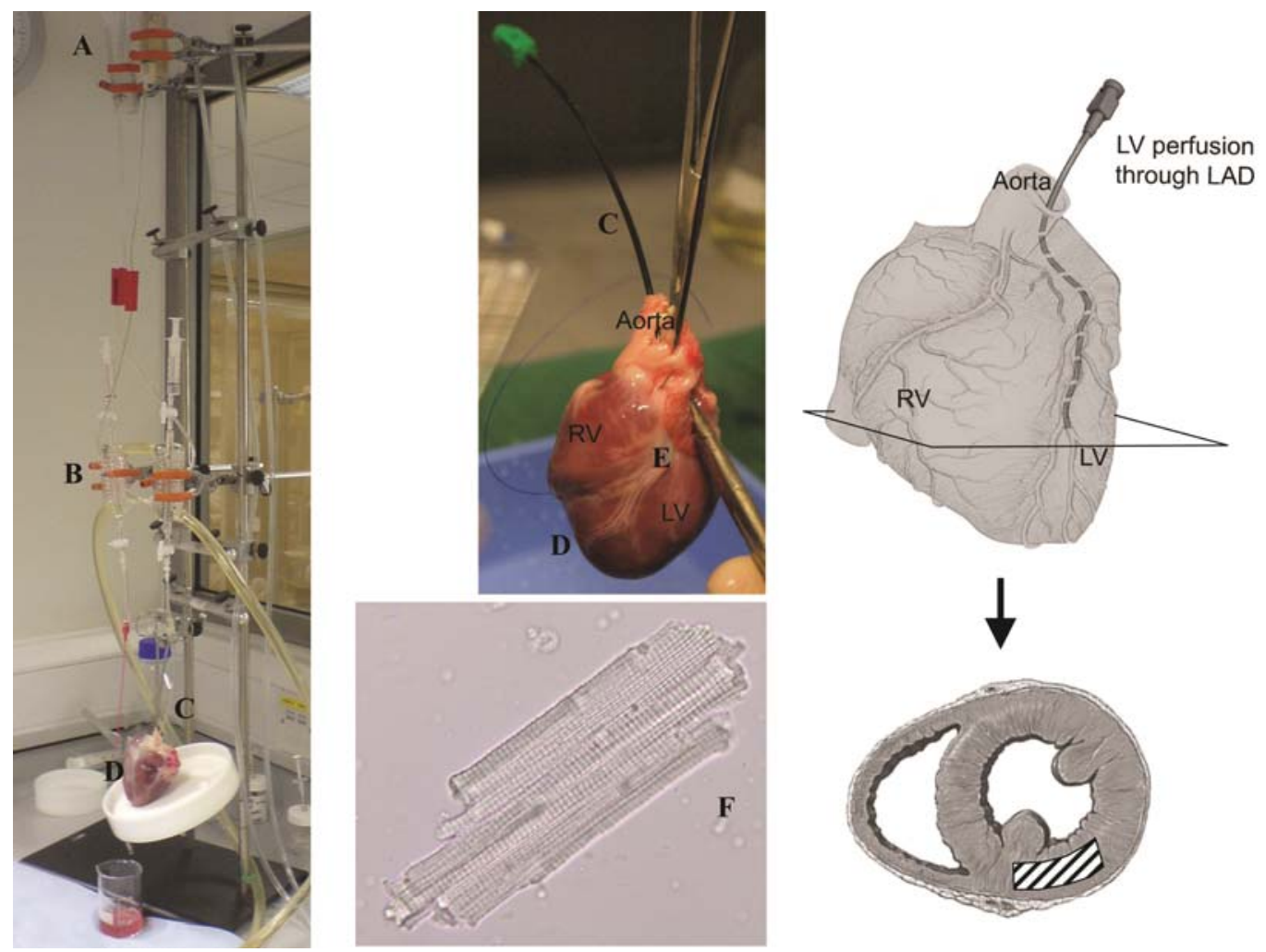

Figure 1: Isolation of canine LVMMs. The left panel shows the apparatus used for isolating LVMM cells from a canine heart. Photographic and schematic showing cannulation (C.) of the left anterior descending (LAD) coronary artery for perfusion of the left ventricle during the isolation procedure (top right). The catheter is placed through the aorta into the LAD (E.) and a photo of a single isolated LVMM (F.). A. indicates the solution reservoir; B. the heated coil; C. the cannula; $\mathbf{D}$. the heart.

\subsubsection{Pre-surgery preparation}

1. The day before isolation prepare the following:

1 liter normal Tyrode's solution containing $0.2 \mathrm{mM} \mathrm{CaCl}_{2}$,

9 liters normal Tyrode's solution containing $1.8 \mathrm{mM} \mathrm{CaCl}_{2}$,

5 liters calcium-free Tyrode's solution.

2. Check the $\mathrm{pH}$ (7.38-7.42) and osmolarity (275-315 mOsm) of the normal Tyrode's solutions. Adjust $\mathrm{pH}$ as needed with $1 \mathrm{M} \mathrm{NaOH}$ or $\mathrm{HCl}$.

If the osmolarity of the solution is not within this range, discard and prepare fresh solution.

3. Fill two 1-liter Schott Duran bottles with calcium-free Tyrode's solution and place in the refrigerator $\left(4^{\circ} \mathrm{C}\right)$.

4. On the day of myocyte isolation, but before obtaining the heart, place the two Schott Duran bottles of calcium-free Tyrode's solution on ice and begin bubbling continuously with medical $\mathrm{O}_{2}(99.5 \%)$. 
5. Set up the work space with the following items:

105-mm surgical scissors, straight, S/S, 140-mm Mayo dissection scissors, straight, B/B, 160-mm medium dissecting forceps, straight, Aesculap Iris dissection forceps,

Sewing needle and thread, 100-, 200-, and 500-ml beakers, Waste tissue bag.

6. Connect a 50-ml Plastika syringe via polyethylene tubing to the heating coil. Fill the syringe with bubbled, room-temperature, calcium-free Tyrode's solution. Remove bubbles from the tubing connected to the heating coil.

7. Set the perfusion rate at $\sim 20 \mathrm{ml} / \mathrm{min}$. This should produce a steady stream from the cannula.

8. Fill two $10-\mathrm{ml}$ syringes with cold calcium-free Tyrode's solution and keep at $4^{\circ} \mathrm{C}$ in a refrigerator.

9. Dissolve $150 \mathrm{mg}$ BSA in $300 \mathrm{ml}$ room-temperature calcium-free Tyrode's solution (final $0.5 \mathrm{mg} / \mathrm{ml} \mathrm{BSA}$ ) and add $1.5 \mu \mathrm{l}$ of $1 \mathrm{M} \mathrm{CaCl}_{2}$.

10. Activate the DC50-B5 heating water circulator for 15 min before sacrificing the animal. Adjust the "set" temperature of the heating coil to $37^{\circ} \mathrm{C}$.

\subsubsection{Obtain LVMMs}

11. Induce anesthesia in a dog using $\sim 45 \mathrm{mg} / \mathrm{kg}$ pentobarbitone and supplementary anaesthetic as necessary until there are no pedal or pupil reflexes. Open the chest via left thoracotomy, remove the heart, and immediately place it in cold, oxygenated calcium-free Tyrode's solution. Rinse the heart three times using more of the same solution.

12. Remove some of the tissue around the aorta and shorten it slightly (by 3 to 5 $\mathrm{mm}$ ), leaving sufficient length for the thread and clamp ( $\sim 5 \mathrm{~mm}$; Figure 1, top center).

13. Cannulate the left anterior descending coronary artery through the aorta

(Figure 1, top center, top right) and infuse $\sim 10 \mathrm{ml}$ cold calcium-free Tyrode's solution through the cannula using a syringe from step 8.

14. Use needle and thread to secure the cannula and then attach the cannula to the isolation setup (Figure 1, top center).

15. Perfuse for 5-10 min with room temperature, oxygenated calcium-free Tyrode's solution at $20 \mathrm{ml} / \mathrm{min}$.

16. During perfusion, dissolve collagenase $A$ at $1.1 \mathrm{mg} / \mathrm{ml}$ into the Tyrode's solution containing BSA. Continue bubbling with oxygen.

17. Perfuse the heart with Tyrode's solution containing BSA and collagenase A for $20 \mathrm{~min}$ at $20 \mathrm{ml} / \mathrm{min}$. 
The initial cold perfusion protects the heart muscle from damage during the period before it is attached to the isolation setup. BSA is used to improve survival and recovery of $\angle V M M S$. Perfusion of warm solution and addition of calcium optimize the activity of collagenase $A$.

18. Perfuse the heart with room temperature, oxygenated normal Tyrode's solution containing $0.2 \mathrm{mM} \mathrm{CaCl}$ for $5-10 \mathrm{~min}$ at $20 \mathrm{ml} / \mathrm{min}$.

19. Remove the heart from the perfusion apparatus and dissect $1-\mathrm{cm}$ chunks from the midmyocardial region of the left ventricular wall (Figure 1, bottom right). Place them in a 50-mm dissecting dish containing $50 \mathrm{ml}$ normal Tyrode's solution with $0.2 \mathrm{mM} \mathrm{CaCl}_{2}$, swirl to rinse, and allow to settle for $\sim 10$ $\min$.

20. Decant supernatant, rinse LVMM cells in $10 \mathrm{ml}$ normal Tyrode's solution containing $0.2 \mathrm{mM} \mathrm{CaCl}_{2}$, and filter through a cell dissociation sieve using $100-\mu \mathrm{m}$ Screens for CD-1.

21. Prepare $90 \mathrm{ml}$ of a $5 \mathrm{mg} / \mathrm{ml}$ BSA solution in normal Tyrode's solution containing $1.8 \mathrm{mM} \mathrm{CaCl}_{2}$.

22. Wash LVMM cells in $90 \mathrm{ml}$ normal Tyrode's solution containing $0.2 \mathrm{mM} \mathrm{CaCl}$ and allow them to settle for $\sim 10 \mathrm{~min}$.

23. Discard supernatant, rinse LVMM cells in $45 \mathrm{ml}$ normal Tyrode's solution containing $1.8 \mathrm{mM} \mathrm{CaCl}_{2}$ and $5 \mathrm{mg} / \mathrm{ml} \mathrm{BSA}$, and then allow to settle for $\sim 10$ min. Repeat.

24. Replace supernatant with $90 \mathrm{ml}$ normal Tyrode's solution containing $1.8 \mathrm{mM}$ $\mathrm{CaCl}_{2}$ and examine the cells under an inverted microscope.

Healthy LVMMs should be quiescent rod-shaped cells with clear cross-striations (Figure 1F). Although quantification is not required for the purpose of this work, one can expect a yield of $\sim 9 \times 10^{6}$ cells.

25. Wash the perfusion setup twice with $70 \%$ ethanol, then twice with deionized, distilled water.

\subsubsection{Prepare Vehicle, di-4-ANEPPS, and Test and Reference Compounds}

26. Dissolve $5 \mathrm{mg}$ di-4-ANEPPS in $100 \%$ ethanol at a concentration of $10 \mathrm{mM}$. Dilute this stock solution in ethanol to a concentration of $1 \mathrm{mM}$, and then dilute $100 \mu \mathrm{l}$ of this solution in 1 liter of normal Tyrode's solution containing $1.8 \mathrm{mM} \mathrm{CaCl}_{2}$ to yield a final $0.1 \mu \mathrm{M}$ dye solution.

IMPORTANT: Minimize exposure of all solutions containing di-4-ANEPPS to light by wrapping in tin foil and, if necessary, placing a tin foil lid over the top. 
27. Dissolve $\sim 25 \mathrm{mg}$ test compound in DMSO at a concentration of $100 \mathrm{mM}$. Dilute this solution serially with DMSO to produce three more DMSO stocks (e.g., 10, 1, and $0.1 \mathrm{mM}$ ). Finally, dilute each test compound stock 1000 -fold in $0.1 \mu \mathrm{M}$ dye solution (final $100,10,1$, and $0.1 \mu \mathrm{M}$ test compound).

28. Dissolve $\sim 25 \mathrm{mg}$ reference compound (e.g., dofetilide) in DMSO at a concentration of $1 \mathrm{mM}$. Dilute this 1000 -fold in $0.1 \mu \mathrm{M}$ dye solution (final $1 \mu \mathrm{M}$ reference compound). The reference compound should be tested in preliminary experiments to determine the optimal concentration for response. This applies to dofetilide or any other reference compound.

29. Dilute DMSO 1000 -fold in $0.1 \mu \mathrm{M}$ dye solution to make a $0.1 \%$ DMSO vehicle solution.

\subsubsection{Prepare Experimental Setup}

30. Turn on the arc lamp power supply, followed by the computer, stand-alone power supply for the Cairn shutter, dual-emission photometry system with amplifiers, 2×PMT power supply modules, monitor, microscope light, vacuum, HSE simulator, and Multiclamp 700A. Set both PMT power supply modules at $920 \mathrm{~V}$.

The Multiclamp 700A is not used, but must be turned on to ensure that the pCLAMP protocol sequencing operates without querying the telegraphed settings within PCLAMP.

31. Clean the microscope objective and place a drop of oil on it.

32. Place a cover glass in the FHD microscope chamber, clip the chamber in place, and make contact between the objective and cover glass so that the LVMMs can be seen.

33. Fill and prime each channel of the CF-8vs valve assembly with $50 \mathrm{ml}$ of each vehicle, reference, and test solution until the perfusion flow is constant and without bubbles. Attach the inflow tube of the Cell MicroControls mTCII temperature heater to the FHD microscope chamber system.

34. Perfuse the FHD microscope chamber with $0.1 \mu \mathrm{M}$ di-4-ANEPPS dye solution at $3 \mathrm{ml} / \mathrm{min}$ until flow is constant. Switch off perfusion and disconnect suction.

\subsubsection{Dye-Load Cells and Record APs}

35. Place $5 \mathrm{ml}$ of $0.1 \mu \mathrm{M}$ dye solution and $2 \mathrm{ml} \operatorname{LVMMs}\left(\sim 2 \times 10^{5}\right.$ cells $)$ into a foil-wrapped vial. Leave for $\sim 10 \mathrm{~min}$ at room temperature.

36. Using a pipet, transfer $1 \mathrm{ml}$ dye-loaded cells to the FHD microscope chamber and allow to settle for $\sim 3$ min to ensure that cells are not washed away when perfusion starts. 
37. Set the temperature of the Cell MicroControls mTCII temperature controller to $37^{\circ} \mathrm{C}$, activate the heater, connect the suction, and perfuse $0.1 \mu \mathrm{M}$ dye solution for $10 \mathrm{~min}$ at $3 \mathrm{ml} / \mathrm{min}$.
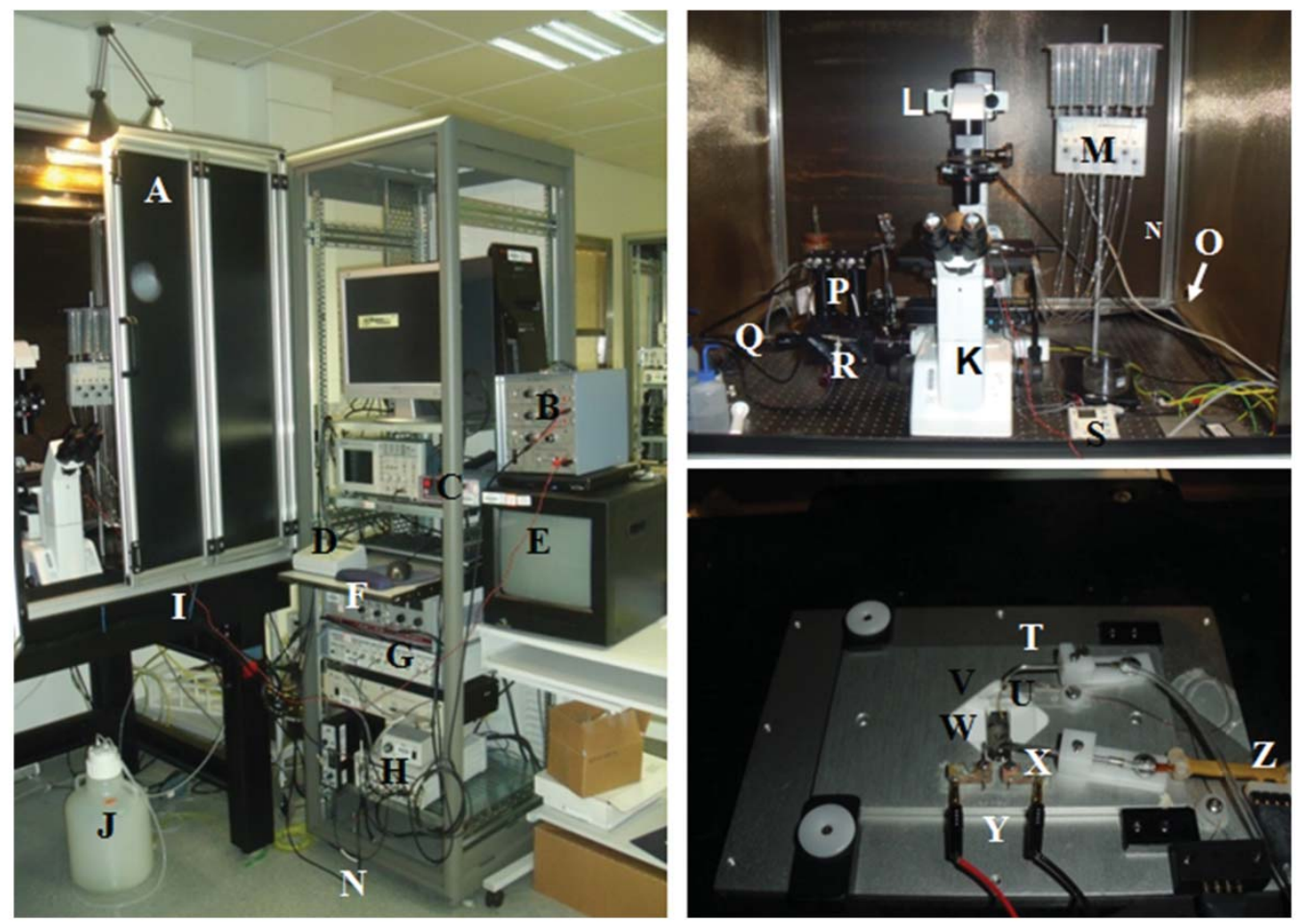

Figure 2: Optical experimental setup and tissue bath. Epifluorescence recordings are made from LVMM cells as previously described (Hardy et al., 2009). The setup includes a Faraday cage (A.), HSE stimulator P (B.), Cairn shutter (C.), cFlow controller (D.), Vista 14-inch monitor (E.),dual-emission photometry system (F.), MultiClamp 700A (G.), Digidata 1322A $(\mathbf{H}$.$) , antivibration table (I.), vacuum waste bottle (J.), inverted microscope (K.), long-pass$ filter (L, >700 nm), CF-8vs valve assembly (M.), superfusion tubing (N.), connection to iris (xenon arc lamp, O.), narrow bandpass filters (P, 540-580 and 600-640 nm), infrared camera (Q.), chromatic reflectors $\left(\mathbf{R}_{,}<590\right.$ and $\left.<700 \mathrm{~nm}\right)$, temperature controller (S.), outflow (T.), thermistor $\left(\mathbf{U}_{\mathbf{.}}\right)$, chamber $\left(\mathbf{V}_{\mathbf{.}}\right)$, oil objective $\left(\mathbf{W}_{\mathbf{.}}\right)$, inflow $\left(\mathbf{X}_{\mathbf{.}}\right)$, electrodes $\left(\mathbf{Y}_{\mathbf{.}}\right)$, and heater (Z.).

38. Using protocol $A$ in Table 1, apply continuous electrical field stimulation at $1 \mathrm{~Hz}$ (i.e., 60 pulses/min) for $4 \mathrm{msec}$ duration using a constant-voltage isolated MyoPacer field stimulator through bath-mounted platinum wire electrodes (FHD microscope chamber; Figure 2). Increase the stimulator voltage amplitude (generally 4-6 V) until some LVMMs begin to contract in time with the stimulus. Select a healthy rod-shaped cell that has visible striations (Figure 1F), is not in contact with any other cells, and contracts in time with the stimulus. Move this LVMM to the center of the microscope field of view. Use a diaphragm placed before the PMTs to limit the view around the cell and ensure that light emitted from neighboring cells does not interfere 
with recordings. Close the rig doors. Determine the stimulation voltage $(\sim 10-$ $20 \%$ above the minimum threshold) that will evoke a contraction in the cell. Stimulation is controlled via protocols written in Clampex, the data acquisition module of pClamp. Protocol A allows for continued stimulation of LVMMs without exposure to excitation light (to avoid di-4-ANEPPS phototoxicity) and with the PMTs gated (to maintain greater stability). This ensures that a valid optical AP signal is evoked.

39. Once a valid optical AP signal has been obtained, use protocol B (Table 1) to acquire an optical AP signal for 5 sec every $4 \mathrm{~min}$. In protocol $\mathrm{B}$, continuous stimulation of LVMMs is maintained at $1 \mathrm{~Hz}$, but the cells are exposed to excitation light and the PMTs are not gated (to allow for quantitative detection of the two different emitted wavelengths).

40. Just before initiating superfusion with the test compound, record APs during exposure to the $0.1 \%$ DMSO vehicle solution. Use this AP as the control.

41. Initiate superfusion with each concentration of test compound by opening the appropriate valve of the CF-8vs valve assembly using the cFlow controller.

42. If the test compound has an effect on the AP (e.g., prolongation or shortening), apply $0.1 \%$ DMSO to test the reversibility of the effect.

43. If the test compound has no effect on the AP, apply the reference compound to test whether the LVMM cell is capable of responding to an active compound.

44. Upon completion of the experiment, switch off perfusion and heater, disconnect suction, stop stimulation, and push the lever of the microscope away and remove the filter.

45. Clean all equipment between experiments. Flush the FHD microscope chamber with ethanol followed by water and then dry thoroughly. Lower the objective, remove the glass cover, clean under the chamber with a tissue, and clean the objective with a lens cleaning tissue. Place sufficient drops of oil on the objective, place a new glass cover in the FHD microscope chamber, and then raise the lens so that a clear image can be seen through the oil via the eyepieces on the microscope when viewing any part of the FHD microscope chamber upon which LVMM cells may be placed.

46. Perform additional experiments to obtain data from at least four additional cells. 
Table 1

\begin{tabular}{|lccc|}
\hline & "Step 44 protocol" & “Step 45 protocol” & $\begin{array}{c}\text { Digidata } \\
\text { 1322A } \\
\text { input/ output }\end{array}$ \\
\hline Stimulus & $5 \mathrm{~V}$ & $5 \mathrm{~V}$ & Digital OUT 3 \\
\hline Shutter control & $0 \mathrm{~V}$ & $5 \mathrm{~V}$ & Digital OUT 2 \\
\hline PMT gating & $\begin{array}{c}5 \mathrm{~V} \\
\text { (which "Gates" the PMT) }\end{array}$ & $0 \mathrm{~V}$ & Digital OUT 1 \\
\hline $\begin{array}{l}560 \mathrm{~nm} \text { PMT } \\
\text { detection }\end{array}$ & Yes & Yes & Analogue IN14 \\
\hline $\begin{array}{l}620 \mathrm{~nm} \text { PMT } \\
\text { detection }\end{array}$ & Yes & Yes & Analogue IN15 \\
\hline Math signal & No & IN14/IN15 & Not applicable \\
\hline
\end{tabular}

Table 1: Protocols used in Clampex 10 software for detection of emitted light.

In both protocols, the acquisition mode is episodic, the sweep duration is $1 \mathrm{sec}$, and the data acquisition is at $10 \mathrm{kHz}$. However, protocol B will only run for 5 sweeps, whereas protocol A will run for 235 sweeps.

\subsubsection{Analyze Data}

47. Using pClamp software, measure AP parameters (Figure 3A) from on-line acquired data and save the relevant information for additional off-line analysis.

48. Perform off-line analysis using Clampfit (a module of pClamp) and a custommade optical AP myocyte macro ${ }^{3}$ to smooth the optical trace data as follows:

a. Calculate a baseline by taking the mean of the first $10 \mathrm{msec}$ (100 data points).

b. Subtract this value from all data points.

c. Collect every tenth data point from the baseline data and smooth using cubic splines.

d. Normalize smoothed and baseline data with respect to the maximum value of the AP "dome" peak.

49. Quantify test compound effects relative to data collected in the presence of the first perfusion with $0.1 \%$ DMSO. Data for each experimental condition are the average of five APs at $1 \mathrm{~Hz}$, collected every $4 \mathrm{~min}$.

50. If appropriate, produce a graph plotting the concentration-effect of the test compound on a given AP parameter (Figure 4). 

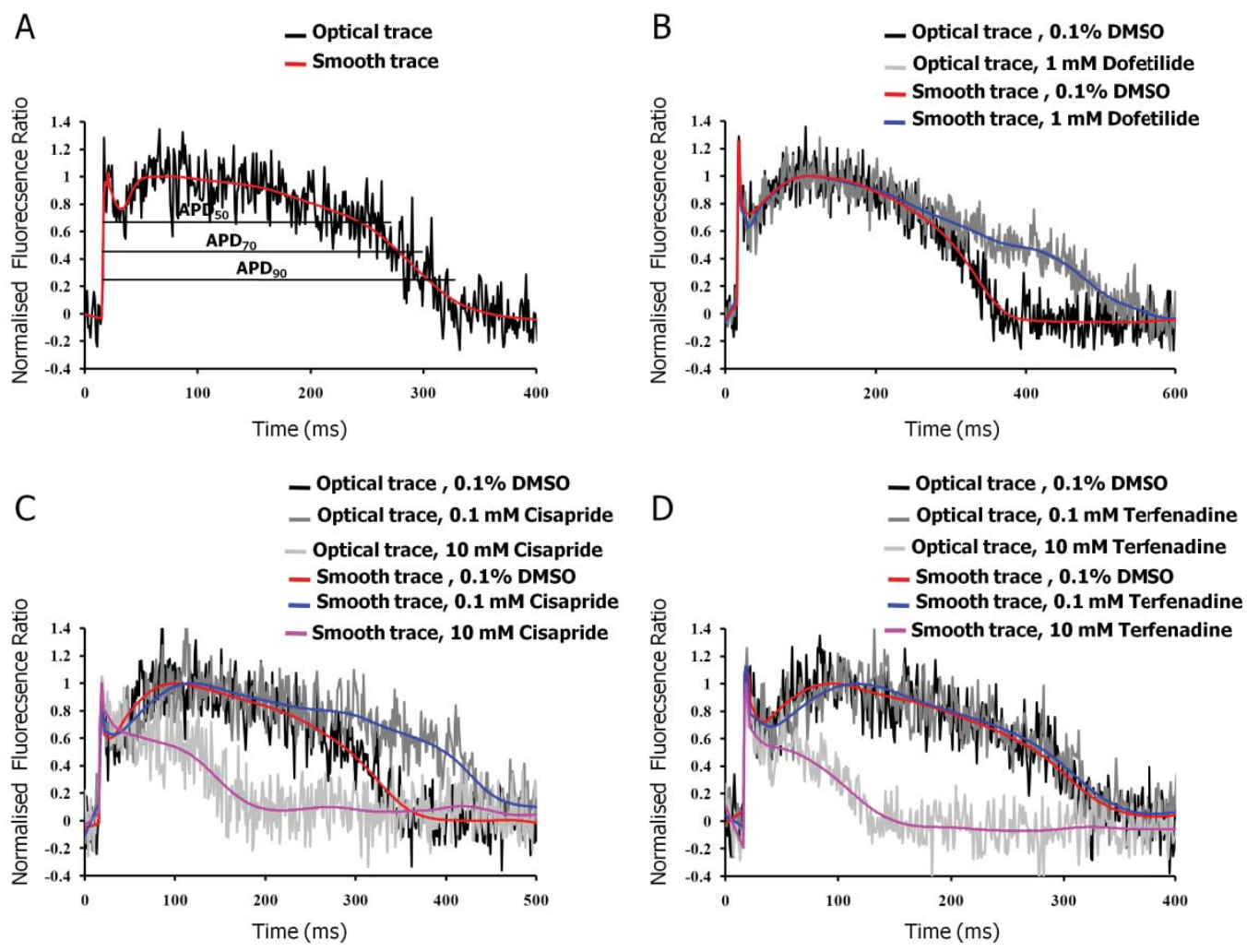

Figure 3: Measurement of changes in the AP during superfusion with reference compounds. A. Representative optical AP of a canine LVMM cell and parameters measured during the experiment. APD50, APD70, and APD90: action potential duration optically measured at 50\%, $70 \%$, and $90 \%$ repolarization. B-D. Representative optical APs recorded using di-4-ANEPPSbased method with vehicle solution ( $0.1 \%$ DMSO) and in the presence of dofetilide (class III antiarrhythmic; $1 \mu \mathrm{M}$ ), cisapride (multiple ion channel blocker; 0.1 and $10 \mu \mathrm{M}$ ), and terfenadine (multiple ion channel blocker; 0.1 and $10 \mu \mathrm{M}$ ). B-D reprinted from Hardy et al., 2009 with permission from Elsevier ${ }^{3}$. 

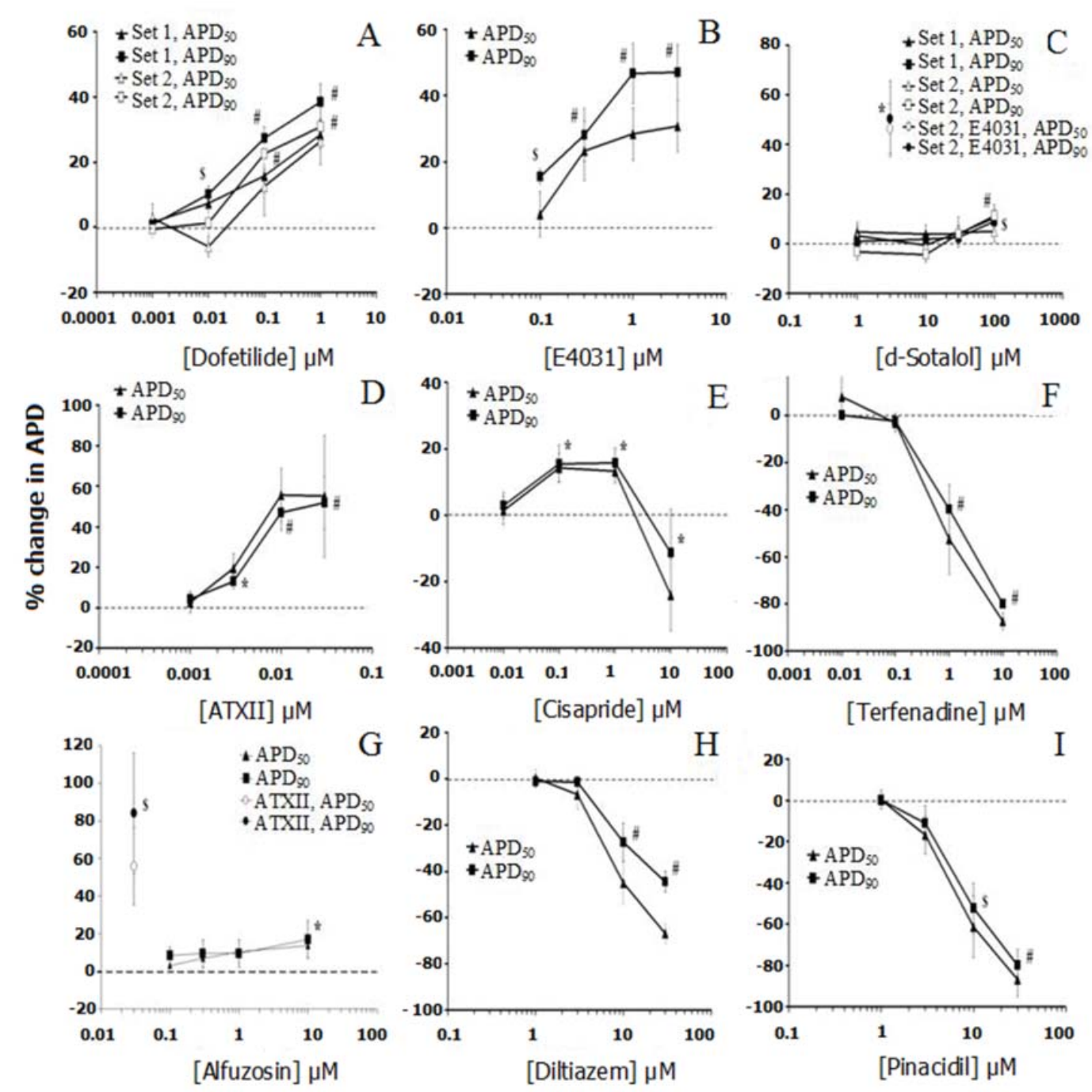

Figure 4: Effects of nine reference compounds on optically measured APD. Graphs show mean $\%$ change in APD50 and APD90 induced by A. dofetilide ( $n=6[1$ dog] for each set), B. E4031 (class III antiarrhythmic; $n=6[1$ dog]), C. D-sotalol (class III antiarrhythmic; $n=8$ [2 dogs] for first set, $n=6$ [1 dog] for second set, $n=3$ [1 dog] for E4031 during second set), D. ATX-II (agonist of late $\left.\mathrm{I}_{\mathrm{Na}} ; \mathrm{n}=5[1 \mathrm{dog}]\right)$, E. cisapride $(\mathrm{n}=5[1 \mathrm{dog}])$, F. terfenadine $(n=5$ [1 dog]), G. alfuzosin (multiple ion channel blocker; $n=6$ [1 dog] and $n=4$ [1 dog] for ATX-II for experiments in which D-sotalol did not have an effect on optically measured APD), H. diltiazem (inhibitor of $\mathrm{I}_{\mathrm{CaL}} ; \mathrm{n}=7$ [2 dogs]), and I. pinacidil (opener of $\mathrm{I}_{\mathrm{KATP}} ; \mathrm{n}=4$ [2 dogs]) at a pacing frequency of $1 \mathrm{~Hz}$. Data expressed as mean \pm SEM. $* \mathrm{P}<0.05, \$ \mathrm{P}<0.01$, and $\# \mathrm{P}<0.001$ compared to values from $0.1 \%$ DMSO. Statistical analysis is to be 'within cell', with each concentration of each reference compound within each cell compared to vehicle ( $0.1 \%$ DMSO). Change from vehicle analyzed and the geometric mean change (\%) from vehicle is to be reported, together with $95 \%$ confidence limits for the mean change. Effects are to be reported as statistically significant if $\mathrm{P}<0.05$. Increases or decreases are to be considered equally likely, leading to a two-sided testing approach. The data are log transformed prior to analysis and each parameter is analyzed separately. All comparisons are with the vehicle group. Reprinted from Hardy et al. with permission from Elsevier ${ }^{3}$. 


\subsection{Arrhythmogenic Effects of a Test Compound on Beat-To-Beat Variability of Repolarization and Occurrence of Early and Delayed After-Depolarizations}

Described in this protocol is a procedure for assessing the arrhythmogenic effects of a test compound on the beat-to-beat variability of APs in LVMMs and the occurrence of early after-depolarizations (EADs) and delayed after-depolarizations (DADs). The conventional microelectrode (sharp-electrode or SE) technique is employed to measure the AP. This technique allows direct measurement of the transmembrane potential over a prolonged period of time.

\subsubsection{Materials}

- Isolated LVMMs (see Basic Protocol)

- Normal Tyrode's solution (see recipe) containing $1.8 \mathrm{mM} \mathrm{CaCl}_{2}$

- Test compound

- Reference compound (e.g., dofetilide, a class III antiarrhythmic agent)

- Dimethyl sulfoxide (DMSO; Sigma)

- $3 \mathrm{M} \mathrm{KCl}$

Equipment and supplies for microelectrode recording of AP (Figure 5), consisting of:

- Antivibration table and Faraday cage (e.g., TMC)

- Inverted microscope (e.g., Nikon Eclipse TE200) with 10x eyepieces and 40x oil objective

- Perfusion chamber for myocytes (e.g., BT-1[-SY], Cell MicroControls)

- Perfusion system with water-jacketed heat exchanger for inflow of superfusate to myocyte chamber (e.g., Radnoti)

- Micromanipulator for coarse and fine positioning of microelectrodes (e.g., Narishige)

- Headstage with microelectrode holder (e.g., Molecular Devices)

- Glass microelectrodes

- Fiber optic light source (e.g., Schott KL1500, Schott)

- Programmable stimulator and computer with software for driving stimulator (e.g., Tabor pulse generator, Tabor Electronics)

- MultiClamp 700A or Axoclamp 2B amplifier, Digidata 1322A digitizer (Molecular Devices)

- Computer with pClamp 10 software (Molecular Devices) for acquisition and analysis of electrical signal

- Video camera and monitor (e.g., Pulnix TM-640, JAI, and CEM-12A-II, CBC)

- Video edge-motion detector (e.g., Crescent Electronics VED-105)

- 3-ml Syringe and 22-G spindle needle (World Precision Instruments, Germany) 

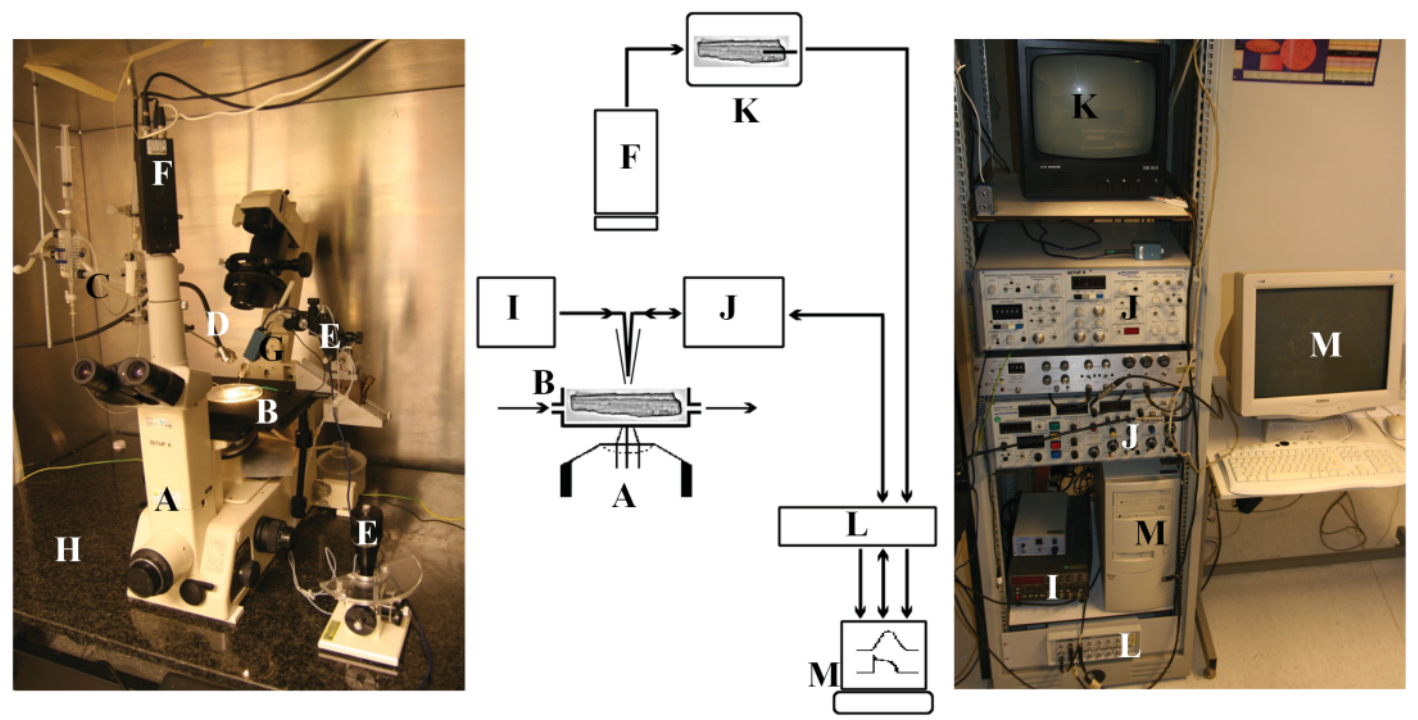

Figure 5: Sharp-electrode experimental setup. The center panel is a schematic drawing of the assembly of components in the outer photographs, including inverted microscope (A.), microscope table with perfusion chamber (B.), perfusion system with water-jacketed heat exchanger for inflow of superfusate (C.), fiber-optic light source (D.), micromanipulators for coarse and fine positioning of microelectrodes (E.), video camera (F.), headstage (preamplifier) with holder and microelectrode (G.), vibration-isolated work station (H.), stimulator (I.), microelectrode amplifiers (J.), video edge-motion detector (K.), analog-todigital converter (L.), and computer (M.) with pCLAMP 10 software for automated waveform control and data acquisition.

\subsubsection{Prepare Vehicle and Test and Reference Compounds}

1. Dissolve $\sim 25 \mathrm{mg}$ test compound in DMSO at a concentration of $100 \mathrm{mM}$. Serially dilute this solution with DMSO to produce three more stocks at 10,1 , and $0.1 \mathrm{mM}$.

2. Dilute the DMSO stocks 1000 -fold in normal Tyrode's solution containing 1.8 $\mathrm{mM} \mathrm{CaCl} 2$ to give final concentrations of $100,10,1$, and $0.1 \mu \mathrm{M}$ test compound.

3. At room temperature, dissolve $\sim 25 \mathrm{mg}$ reference compound (e.g., dofetilide) in DMSO at a concentration of $1 \mathrm{mM}$. Dilute this stock in normal Tyrode's solution containing $1.8 \mathrm{mMCaCl}_{2}$ to yield a final concentration of $1 \mu \mathrm{M}$ reference compound.

The reference compound should be tested in preliminary experiments to determine the optimal concentration for response. This applies to dofetilide or any other reference compound.

4. At room temperature, dilute DMSO 1000 -fold in normal Tyrode's solution containing $1.8 \mathrm{mM} \mathrm{CaCl}_{2}$ to yield a $0.1 \%$ DMSO vehicle solution. Prepare experimental setup and record APs. 
5. Turn on microscope, light source, computer for data acquisition, stimulator, video edge system, and monitor.

6. Set temperature to $37^{\circ} \mathrm{C}$, activate the heater, connect the suction, and start perfusion of normal Tyrode's solution containing $1.8 \mathrm{mM} \mathrm{CaCl}_{2}$ at $3 \mathrm{ml} / \mathrm{min}$. Leave for $10 \mathrm{~min}$ to reach target temperature.

7. Turn off perfusion and place $\sim 1 \mathrm{ml}$ of LVMMs into the perfusion chamber. Leave for 5 to $10 \mathrm{~min}$ to allow cells to adhere to the bottom of the chamber.

8. Resume perfusion at $3 \mathrm{ml} / \mathrm{min}, 37^{\circ} \mathrm{C}$.

9. Fill a glass microelectrode with $3 \mathrm{M} \mathrm{KCl}$ using a 3-ml syringe and a 22-G spindle needle.

The glass microelectrodes are made from filamented capillary tubes (World Precision Instruments) using a single-barreled microelectrode puller (e.g., DMZ universal puller, Zeitz-Instruments Vertriebs). The resistance should be 30-60 MS.

10. Insert the filled microelectrode into the microelectrode holder attached to the micromanipulator.

11. Place the tip of the microelectrode into the superfusion bath using the micromanipulator and under microscopic guidance. Zero the amplifier using the acquisition software and start recording.

12. Select a single undamaged rod-shaped cell with visible striations that is not in contact with any other cells and bring to the center of the microscopic view.

13. Using the micromanipulator, gently move the microelectrode so it is above the center of the cell of interest (without touching it).

14. Check that the amplifier is still at $0 \mathrm{mV}$ and readjust, if necessary.

15. Gently lower the microelectrode onto the cell of interest. Once contact is made, record the membrane voltage of the cell.

The membrane voltage should decrease to around $-80 \mathrm{mV}$ if the cell is healthy and there is good contact. If the voltage does not decrease to $-80 \mathrm{mV}$ within 5 to 10 min, apply a hyperpolarizing pulse of 20 to $40 \mathrm{mV}$ to help seal the microelectrode around the cell. After several minutes, remove the hyperpolarizing pulse and monitor the cell for a stable resting membrane potential. If the resting membrane potential is not stable, locate and impale another cell.

16. Once a stable resting membrane potential of around $-80 \mathrm{mV}$ is attained, start the stimulator at a pacing frequency of $1 \mathrm{~Hz}$ (1000-msec cycle length) with a stimulus duration of 5 msec. 
This should initiate a cardiac AP. If no AP is observed, increase the stimulation duration or amplitude. If the AP morphology is suboptimal (e.g., no spike, dome shape characteristics) locate another cell and impale it. See Fig 6 for examples of optimal SE APS.

17. Move the markers of the video edge detection to the end of the cell to facilitate recording of the contractions.

18. After a 5- to 10-min stabilization period, decrease the pacing frequency to 0.5 $\mathrm{Hz}$. Allow another stabilization period of $\sim 5 \mathrm{~min}$, then increase the pacing frequency to $2 \mathrm{~Hz}$.

This will make it possible to determine any rate dependence of the test compound.

19. Just before initiating superfusion with the test compound, record APs during exposure to the $0.1 \%$ DMSO vehicle solution, and continue until a stable recording is obtained at each cycle length. Use these APs as the controls.

20. Initiate superfusion with each concentration of the test compound and record APs at each of the three pacing frequencies.

21. If there is an effect of the test compound on the AP, apply $0.1 \%$ DMSO to test the reversibility of the effect after examining all concentrations of the test compound.

22. If the test compound has no effect on the AP, apply a reference compound to test whether the LVMM is capable of responding to an active compound. If the study is designed to assess the potential of the test compound to prolong the QT interval, use dofetilide as the reference compound. 
A
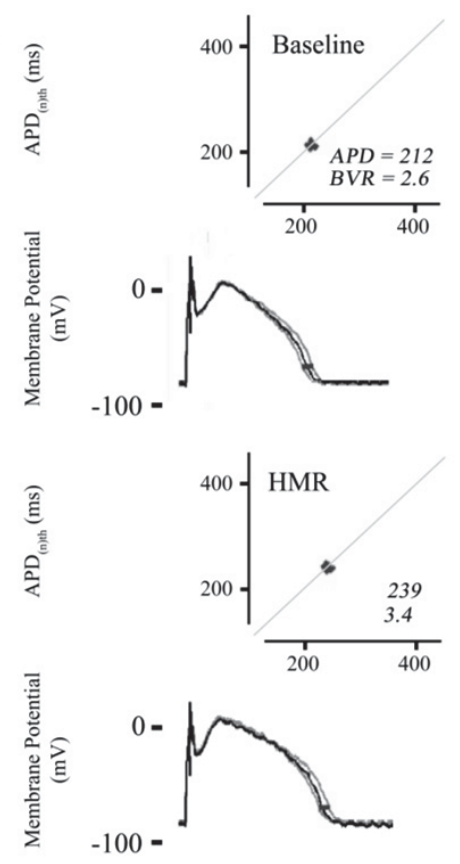

B
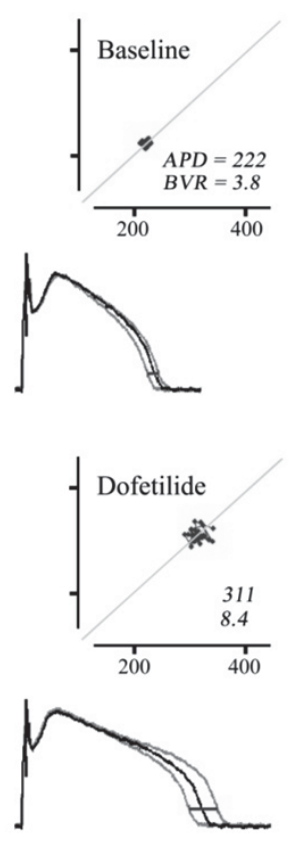
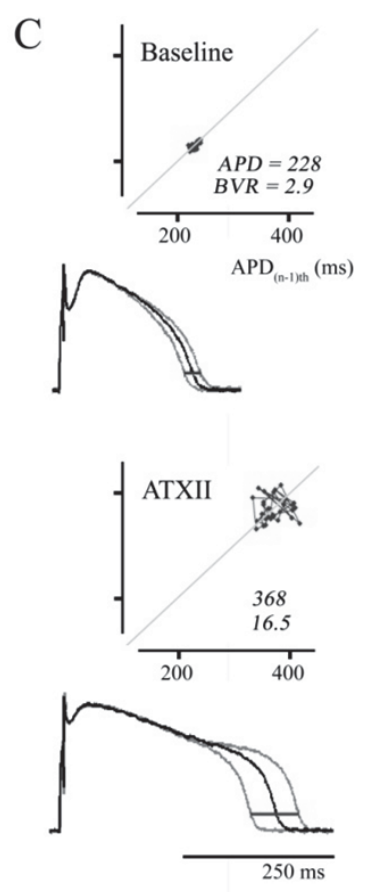

Figure 6: Effects of test compounds on APs recorded from a canine LVMM using the SE technique. Effects of (A.) HMR1556 (inhibitor of $\mathrm{I}_{\mathrm{Ks}} ; 0.5 \mu \mathrm{M}$ ), (B.) dofetilide (class III antiarrhythmic; $1 \mu \mathrm{M}$ ), and (C.) ATX-II (augmenter of late $\mathrm{I}_{\mathrm{Na}} ; 0.02 \mu \mathrm{M}$ ) on BVR and APD90 at $1 \mathrm{~Hz}$ pacing frequency. Representative AP recordings showing the minimum, maximum, and median beats are shown foreach condition. Poincaré plots for each condition are also shown. Reprinted from Johnson et al., 2010 with permission from Elsevier ${ }^{7}$.

23. At the end of the experiment, turn off the perfusion, stimulator, and heater.

24. Clean all equipment between experiments. Rinse the microscope chamber and perfusion tubing with $100 \%$ ethanol and distilled water to prevent contamination and remove residual myocytes.

25. Begin next series of experiments to obtain data from at least four additional cells.

\section{Analyze Data}

26. Using pClamp software, measure AP parameters from on-line acquired data and save the relevant information for additional off-line analysis.

27. Perform additional off-line analysis using Clampfit ${ }^{7,9}$.

28. Quantify test compound effects relative to the $0.1 \%$ DMSO vehicle data and, if appropriate, produce a graph plotting the concentration-effect of the test compound for a given AP parameter (Figure 4). Data for each experimental condition are the mean of 30 action potentials at each pacing frequency at steady state.

29. Calculate the BVR of these same beats using the following formula:

$$
\text { BVR }=\Sigma(\text { APD90 } n-A P D 90 n-1) /(30 \times \sqrt{ } 2)
$$


Poincare graphs, plotting the APD of beat $n$ versus beat $n-1$ (Figures 6 and 7), can also be made to visualize these data. If EADs are observed with the test compound, BVR should be assessed before the first one is noted.

30. Count the number of EADs or DADs that are seen in a 30-beat segment, and then calculate the percentage of beats showing these abnormalities.

The number of EADs and DADs under the various conditions provides an additional proarrhythmic marker, as EADs have been shown to play a role in arrhythmogenesis (Figure 7).

\subsection{Arrhythmogenic Effects of a Test Compound on Beat-To-Beat Variability of Repolarization Under Conditions that Mimic Long QT1 in the Presence of $\beta$-Adrenergic Stimulation}

Described below is a procedure for assessing the arrhythmogenic effects of a test compound on the beat-to-beat variability of APs in LVMMs and occurrence of EADs and DADs in the presence of $\beta A R$ stimulation using ISO. ISO is recommended due to its high selectivity for $\beta$-adrenergic versus a-adrenergic receptors, and because the majority of currently available data has been obtained using ISO. The microelectrode technique is employed for measuring the AP.

Additional Materials (also see Alternate Protocol 1).

$10 \mathrm{mM}$ ISO (Sigma) in $30 \mu \mathrm{M}$ ascorbic acid (prepare immediately before use and store in the dark at $4^{\circ} \mathrm{C}$ ).

NOTE: Stock and working solutions of ISO should be wrapped in tin foil and kept closed whenever possible to prevent degradation.

1. Place $150 \mathrm{ml}$ normal Tyrode's solution containing $1.8 \mathrm{mM} \mathrm{CaCl}_{2}$ into each of two 200-ml flasks at room temperature.

2. Dissolve a DMSO stock of test compound at 1000 -fold in one flask. In the other, dissolve the same concentration of test compound plus $100 \mathrm{nM}$ ISO.

3. At room temperature, dilute DMSO 1000 -fold in normal Tyrode's solution containing $1.8 \mathrm{mM} \mathrm{CaCl}_{2}$ to yield a $0.1 \%$ DMSO vehicle solution.

4. Prepare electrophysiological equipment and establish recordings of the resting membrane potential as described in Alternate Protocol 1 (steps 5 to 18).

5. Just before initiating superfusion with the test compound, record APs during exposure to $0.1 \%$ DMSO vehicle solution. Use this AP as the control.

6. Initiate superfusion with the test compound and record APs at each of the three pacing frequencies. 
7. Once steady-state recordings have been made with the test compound at 0.5 , 1 , and $2 \mathrm{~Hz}$, begin superfusion with test compound plus $100 \mathrm{nM}$ ISO while stimulating the cell at $1 \mathrm{~Hz}$.

This should lead to an increase in cell contraction. If no increase is seen, prepare a new ISO solution and repeat. If this still does not lead to an increase in contraction, repeat the protocol with a new cell.

8. Wait until a steady-state recording has been achieved (5-10 $\mathrm{min}$ ) and record APs at $0.5 \mathrm{~Hz}$ followed by $2 \mathrm{~Hz}$. Be sure to wait until a steady state is achieved at each pacing frequency.

9. At the end of the experiment, turn off the perfusion, stimulator, and heater. 10. Clean all equipment between experiments. Rinse the microscope chamber and perfusion tubing with $100 \%$ ethanol and distilled water to prevent contamination and remove residual myocytes.

APs can be analyzed as described in Alternate Protocol 1 (steps 26-29). An example of the effects of ISO after blockade of the $I_{K S}$ channel with the reference compound HMR1556 is shown in Figure 7.

11. Begin next series of experiments to obtain data from at least four additional cells. 

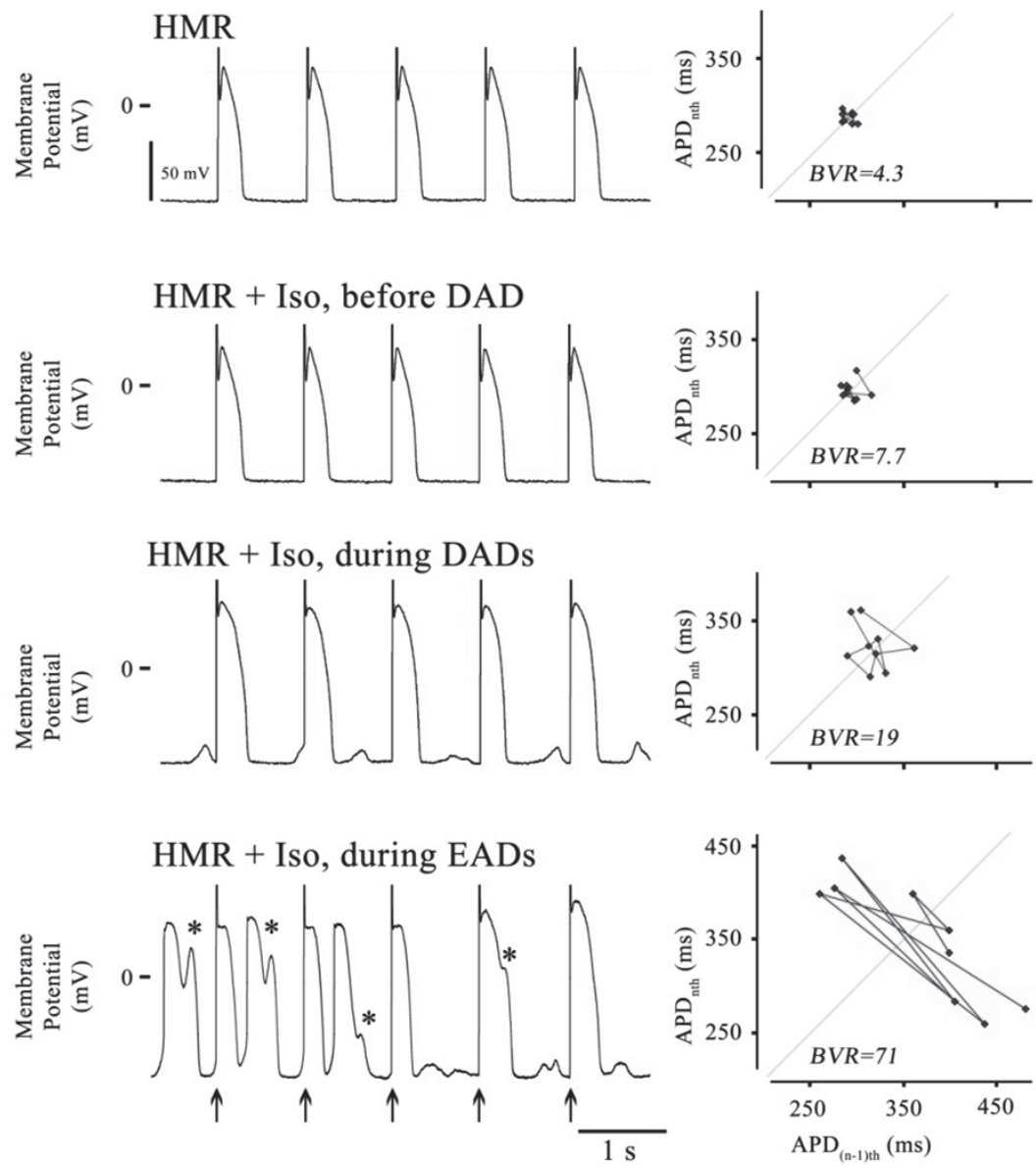

Figure 7: Effect of $\beta A R$ stimulation on a canine LVMM after blockade of $\mathrm{I}_{\mathrm{Ks}}$ current with 0.5 $\mu \mathrm{M}$ HMR1556. Upper panel shows consecutively paced APs at $500 \mathrm{~ms} \mathrm{CL}$, together with Poincaré plots during initial perfusion of HMR1556. Addition of $\beta A R$ stimulation increases APD and BVR before DADs are seen (2nd panel from above). The occurrence of DADs (3rd panel) increases APD and BVR further, and DAD triggered APs and EADs are subsequently observed (4th panel). Arrows indicate timings of paced beats, and $*$ indicate EADs. Low amplitude EADs are also generated under these conditions.Reprinted from Johnson et al., 2010 with permission from Elsevier ${ }^{\text {? }}$.

\section{REAGENTS AND SOLUTIONS}

Use deionized, distilled water (Millipore) in all recipes and protocol steps.

Normal Tyrode's solution

- $10 \mathrm{mM}$ HEPES, pH 7.38-7.42.

- $145 \mathrm{mM} \mathrm{NaCl}$.

- $4 \mathrm{mM} \mathrm{KCl}$.

- $1 \mathrm{mM} \mathrm{MgCl} 2$

- $0,0.2$, or $1.8 \mathrm{mM} \mathrm{CaCl}_{2}$ (as specified).

- $11.1 \mathrm{mM}$ glucose.

- Filter sterilize (e.g., Millipore Steritop).

- Store up to 1 week at room temperature. 
The $\mathrm{pH}$ and osmolarity of these solutions should be between 7.38-7.42 (can be adjusted with $1 \mathrm{M} \mathrm{NaOH}$ or $1 \mathrm{M} \mathrm{HCl}$ ) and 275-315 mOsm. If the osmolarity is not within these limits, the solutions should be discarded. These values must be checked each day before the solutions are used.

\subsection{Commentary}

\subsubsection{Background I nformation}

Compounds that induce TdP have been a major focus of regulatory agencies and the pharmaceutical industry since the mid-1990s ${ }^{10},{ }^{11}$, leading to the adoption of ICH S7B and ICH E14 guidance documents ${ }^{1,2}$. Both of these directives rely on prolongation of the QT interval as a biomarker to predict the risk that a test agent may produce $\mathrm{TdP}^{12}$. As the distribution of ion channel proteins and ionic currents that determine AP shape and duration are similar in both canine and human ventricles ${ }^{13}$, the beagle dog is a commonly used preclinical species for testing the effects of compounds on cardiac repolarization ${ }^{14}$. Additionally, as repolarization of LVMMs usually determines the end of the T-wave ${ }^{5}$, data derived from these cells are thought to be good predictors of possible effects on the human QT interval. This unit describes a di-4-ANEPPS dye-based method (Basic Protocol) for assessing the effects of test compounds on the AP in canine LVMM cells that can be used to more efficiently generate APD data ${ }^{3}$. This makes it possible to discard at an earlier stage in the drug discovery process those test agents that may affect the QT. Because scientists have sought to study the AP at the molecular level by implementing medium-throughput electrophysiological techniques (e.g., IonWorks ${ }^{15-}{ }^{17}$ ), and because the di-4-ANEPPS-based method can generate APD data more easily, this method can help establish an ion channel-APD profile earlier in the drug discovery process than has been possible in the past. In addition to APD assessment, the direct effects of test compounds on cardiac contractility and calcium transients can be detected in canine LVMM cells. Although significant advances have been made in developing in vitro tools to identify the functional effects associated with test compounds, in vitro screens for test compounds that are directly cytotoxic to the heart have not been established. Therefore, canine LVMM cells can also be used to develop in vitro 'black box' assays to predict cardiotoxicity at an earlier stage of a drug discovery program. An additional protocol included in this unit describes a method for investigating the arrhythmogenic effects of a test compound on temporal BVR quantified as STV, as well as the occurrence of EADs and DADs after application of various test compounds (Alternate Protocol 1). This approach is expanded in Alternate Protocol 2 by including $\beta A R$ stimulation, which is a particularly important issue when a test compound may have the propensity to block the $I_{\mathrm{Ks}}$ current. That is, blockade of the $\mathrm{I}_{\mathrm{Ks}}$ channel alone may not, by itself, lead to repolarization prolongation; however, $\beta$ AR stimulation during $I_{\mathrm{Ks}}$ blockade can lead to 
development of EADs and DADs, both of which have been implicated in the generation of arrhythmias ${ }^{7,18}$. Hence, when assessing the properties of a test agent that may block the $\mathrm{I}_{\mathrm{Ks}}$ current, it is critical to examine its effects in the presence and absence of sympathetic stimulation. Although the compound throughput of these alternate protocols is less than with the optical recordings, they provide critical information that cannot be gathered from optical or ion channel screening. For a number of years QT prolongation has been employed as a surrogate for tachyarrhythmia, TdP. However, it is now appreciated that QT prolongation per se does not predict the occurrence of this type of arrhythmia ${ }^{6,19,20}$. Hence, there is a need for other markers for drug-induced arrhythmias. Beat-to-beat variability of repolarization (BVR) has been proposed as one such marker for $\mathrm{TdP}^{6}$. Although the mechanisms responsible for increasing BVR are unknown, it appears they are generated, at least in part, in the cardiac myocyte. Moreover, cellular BVR has been shown to discriminate compounds that are proarrhythmic from those that may safely prolong repolarization 7,21 . The alternate protocols presented here allow for assessment of the effects of test compounds on this parameter as an indicator to how they may influence the temporal variability of repolarization in vivo.

\subsubsection{Critical Parameters}

\section{Preparation of $\angle V M M S$}

Careful preparation of LVMMs is the most critical step when measuring APs in isolated myocytes. Although the placement of the heart in ice-cold oxygenated calcium-free Tyrode's solution helps preserve it from injury, irreversible damage can be caused by taking too long to cannulate the LAD coronary artery and mount the heart before beginning perfusion of warmed, oxygenated normal Tyrode's solution without $\mathrm{CaCl}_{2}$ (Figure 1). Additionally, although calcium-free normal Tyrode's solution containing collagenase A and BSA is perfused for $\sim 20$ min through a beagle dog heart of "normal" size (80-120 g) to accurately digest the perfused area of the left ventricular wall, the time of digestion must be tailored to the size of the heart if it is abnormally large or small. If the size of the heart is not carefully assessed after its removal, the cardiac tissue is likely to be under- or over-digested, yielding cells of lower quality for testing. 


\section{Pacing frequency}

A crucial parameter is the pacing frequency applied to the cardiac myocytes, as described in Alternate Protocols 1 and 2. Many compounds that cause ventricular arrhythmias show reverse rate dependence. That is, they have a greater effect at slower pacing frequencies. To test for this, compounds are examined at multiple pacing frequencies. Caution must be exercised at every stage of recording to ensure that a steady state is attained. Initial pacing of the cell under control conditions should last at least 5 to $10 \mathrm{~min}$ to allow for stabilization with the microelectrode in place. After changing the pacing rate, at least $5 \mathrm{~min}$ should be allowed to achieve steady state.

\section{Choice of test compound concentrations}

At a relatively early stage of drug discovery, the concentration range employed for testing a compound should be based on its effects on five key ventricular ion channels expressed in cell lines: hNav1.5 (mimicking the $\mathrm{hI}_{\mathrm{Na}}$ current), hCav1.2 (mimicking the $\mathrm{hI}_{\mathrm{CaL}}$ current), Kv4.3-hKChIP2.2 (mimicking the $\mathrm{hI}_{\mathrm{To}}$ current), hKv7.1hKCNE1 (mimicking the $\mathrm{hI}_{\mathrm{Ks}}$ current), and hKv11.1 (mimicking the $\mathrm{hI}_{\mathrm{kr}}$ current). These values are obtained in preliminary functional assessments using either conventional or automated electrophysiology. There needs to be consensus on how to use these data to understand APD/QT risk, particularly when a compound modifies a range of mutually compensatory electrophysiological processes, hence the requirement for assessment of a novel compound in an integrated AP system. Thus, there would be considerable value in being able to make full use of the early ion channel potencies with optical AP data that can be used to prioritize compounds early in the drug discovery process (Lead Identification and Lead Optimization) and before substantial resources have been invested. The microelectrode AP study can be used to assess the proarrhythmic potential of one to two lead compounds before they are selected for preclinical development. Other considerations are the solubility of the test compound in the normal Tyrode's solution containing $1.8 \mathrm{mM} \mathrm{CaCl}_{2}$ and the necessity of having the lowest test concentration approximate the predicted free $\mathrm{C}_{\max }$ in humans. The concentrations must be spaced at either half or full $\log _{10}$ intervals to provide a high resolution concentration-effect curve in the event that effects are noted (Figure $\mathbf{4}$ and Table 2).

\section{Troubleshooting}

Table 3 details some common problems encountered while obtaining optical and SE measurements of cardiac APs in isolated LVMM cells. Possible causes and remedies are also discussed. 
Table 2

\begin{tabular}{|c|c|c|c|c|c|c|c|c|}
\hline \multirow{2}{*}{\begin{tabular}{|l} 
Compound \\
Dofetilide
\end{tabular}} & \multirow{2}{*}{$\begin{array}{c}N \\
\text { (animals) }\end{array}$} & \multirow{2}{*}{ Pacing } & \multirow[t]{2}{*}{ Parameter } & \multirow[t]{2}{*}{ Baseline } & \multicolumn{4}{|c|}{ Nominal concentrations tested } \\
\hline & & & & & $0.001 \mu \mathrm{M}$ & $0.01 \mu \mathrm{M}$ & $0.1 \mu \mathrm{M}$ & $1 \mu \mathrm{M}$ \\
\hline & \multirow[t]{6}{*}{$4-6(2)$} & $1.0 \mathrm{~Hz}$ & $\mathrm{APD}_{90}(\mathrm{~ms})$ & $377 \pm 18$ & $0.4 \pm 2 \%$ & $15 \pm 6 \% *$ & $46 \pm 12 \% *$ & $37 \pm 12 \% *$ \\
\hline & & & $\begin{array}{c}\operatorname{STV}\left(A D_{90}\right) \\
(\mathrm{ms})\end{array}$ & $12 \pm 2$ & $11 \pm 2$ & $27 \pm 13$ & $51 \pm 15^{*}$ & $33 \pm 5^{*}$ \\
\hline & & & Triangulation & $1.19 \pm 0.03$ & $1.18 \pm 0.02$ & $1.17 \pm 0.02$ & $1.19 \pm 0.02$ & $1.16 \pm 0.02$ \\
\hline & & $0.5 \mathrm{~Hz}$ & $\mathrm{APD}_{90}(\mathrm{~ms})$ & $436 \pm 25$ & $-3 \pm 2 \%$ & $11 \pm 5 \% *$ & $36 \pm 13 \% *$ & $74 \pm 20 \% *$ \\
\hline & & & $\begin{array}{c}\operatorname{STV}\left(\mathrm{APD}_{90}\right) \\
(\mathrm{ms})\end{array}$ & $16 \pm 4$ & $15 \pm 3$ & $21 \pm 5$ & $38 \pm 8^{*}$ & $92 \pm 19 * \$$ \\
\hline & & & Triangulation & $1.16 \pm 0.03$ & $1.15 \pm 0.02$ & $1.13 \pm 0.02$ & $1.13 \pm 0.02$ & $1.11 \pm 0.01$ \\
\hline \multirow[t]{7}{*}{ D-sotalol } & \multirow{7}{*}{$4(3)$} & & & & $1 \mu \mathrm{M}$ & $10 \mu \mathrm{M}$ & $30 \mu \mathrm{M}$ & $100 \mu \mathrm{M}$ \\
\hline & & $1.0 \mathrm{~Hz}$ & $\mathrm{APD}_{90}(\mathrm{~ms})$ & $364 \pm 18$ & $-4 \pm 2 \%$ & $12 \pm 3 \% *$ & $14 \pm 4 \% *$ & $28 \pm 3 \%{ }^{\#}$ \\
\hline & & & $\begin{array}{c}\operatorname{STV}\left(A^{A P D_{90}}\right) \\
(\mathrm{ms})\end{array}$ & $8 \pm 1$ & $7 \pm 1$ & $13 \pm 1 *$ & $13 \pm 1 *$ & $20 \pm 2^{\#}$ \\
\hline & & & Triangulation & $1.20 \pm 0.04$ & $1.18 \pm 0.03$ & $1.21 \pm 0.05$ & $1.21 \pm 0.04$ & $1.22 \pm 0.06$ \\
\hline & & $0.5 \mathrm{~Hz}$ & $\mathrm{APD}_{90}(\mathrm{~ms})$ & $427 \pm 27$ & $-9 \pm 4 \%$ & $2 \pm 1 \%$ & $22 \pm 7 \% *$ & $37 \pm 6 \%{ }^{\#}$ \\
\hline & & & $\begin{array}{c}\operatorname{STV}\left(A P D_{90}\right) \\
(\mathrm{ms})\end{array}$ & $12 \pm 1$ & $10 \pm 2$ & $13 \pm 3$ & $22 \pm 4 * \$$ & $33 \pm 7 *$ \\
\hline & & & Triangulation & $1.26 \pm 0.1$ & $1.18 \pm 0.02$ & $1.18 \pm 0.03$ & $1.19 \pm 0.05$ & $1.16 \pm 0.05$ \\
\hline \multirow[t]{7}{*}{ DL-sotalol } & \multirow{7}{*}{$7(1)$} & & & & $1 \mu \mathrm{M}$ & $10 \mu \mathrm{M}$ & $30 \mu \mathrm{M}$ & $100 \mu \mathrm{M}$ \\
\hline & & $1.0 \mathrm{~Hz}$ & $\mathrm{APD}_{90}(\mathrm{~ms})$ & $348 \pm 12$ & $-3 \pm 1 \%$ & $4 \pm 3 \%$ & $5 \pm 3 \%$ & $15 \pm 5 \% *$ \\
\hline & & & $\begin{array}{l}\operatorname{STV}\left(\mathrm{APD}_{90}\right) \\
(\mathrm{ms})\end{array}$ & $7 \pm 1$ & $6 \pm 1$ & $8 \pm 2$ & $7 \pm 1$ & $12 \pm 4$ \\
\hline & & & Triangulation & $1.30 \pm 0.06$ & $1.26 \pm 0.05$ & $1.25 \pm 0.04$ & $1.24 \pm 0.04$ & $1.24 \pm 0.04$ \\
\hline & & $0.5 \mathrm{~Hz}$ & $\mathrm{APD}_{90}(\mathrm{~ms})$ & $400 \pm 12$ & $-5 \pm 2 \%$ & $-1 \pm 2 \%$ & $4 \pm 4 \%$ & $18 \pm 9 \% *$ \\
\hline & & & $\begin{array}{c}\operatorname{STV}\left(A^{A P D} D_{90}\right) \\
(\mathrm{ms})\end{array}$ & $9 \pm 2$ & $8 \pm 2$ & $10 \pm 1$ & $12 \pm 2$ & $13 \pm 2$ \\
\hline & & & Triangulation & $1.30 \pm 0.07$ & $1.26 \pm 0.05$ & $1.23 \pm 0.05$ & $1.23 \pm 0.04$ & $1.22 \pm 0.05$ \\
\hline \multirow[t]{7}{*}{ Cisapride } & \multirow{7}{*}{$4-10(2)$} & & & & $0.01 \mu \mathrm{M}$ & $0.1 \mu \mathrm{M}$ & $1 \mu \mathrm{M}$ & $10 \mu \mathrm{M}$ \\
\hline & & $1.0 \mathrm{~Hz}$ & $\mathrm{APD}_{90}(\mathrm{~ms})$ & $360 \pm 15$ & $3 \pm 3 \%$ & $37 \pm 11 \%{ }^{\#}$ & $23 \pm 5 \%{ }^{\#}$ & $-12 \pm 6 \%$ \\
\hline & & & $\begin{array}{c}\operatorname{STV}\left(A^{A P D} D_{90}\right) \\
(\mathrm{ms})\end{array}$ & $7 \pm 1$ & $8 \pm 1$ & $31 \pm 13^{*}$ & $10 \pm 2$ & $5 \pm 1$ \\
\hline & & & Triangulation & $1.22 \pm 0.04$ & $1.22 \pm 0.05$ & $1.28 \pm 0.07$ & $1.29 \pm 0.07$ & $1.47 \pm 0.10 *$ \\
\hline & & $0.5 \mathrm{~Hz}$ & $\mathrm{APD}_{90}(\mathrm{~ms})$ & $380 \pm 19$ & $7 \pm 2 \% *$ & $46 \pm 8 \%{ }^{\#}$ & $69 \pm 19 \% * \$$ & $-16 \pm 6 \%$ \\
\hline & & & $\begin{array}{c}\operatorname{STV}\left(A^{A P D_{90}}\right) \\
(\mathrm{ms})\end{array}$ & $7 \pm 2$ & $12 \pm 2$ & $26 \pm 5^{*}$ & $42 \pm 20 *$ & $17 \pm 9$ \\
\hline & & & Triangulation & $1.14 \pm 0.01^{\$}$ & $1.14 \pm 0.01$ & $1.15 \pm 0.01$ & $1.22 \pm 0.04$ & $1.36 \pm 0.09 *$ \\
\hline
\end{tabular}




\begin{tabular}{|c|c|c|c|c|c|c|c|c|}
\hline \multirow{2}{*}{$\begin{array}{l}\text { Compound } \\
\text { Terfenadine }\end{array}$} & \multirow{2}{*}{$\begin{array}{c}N \\
\text { (animals) }\end{array}$} & \multirow{2}{*}{ Pacing } & \multirow[t]{2}{*}{ Parameter } & \multirow[t]{2}{*}{ Baseline } & \multicolumn{4}{|c|}{ Nominal concentrations tested } \\
\hline & & & & & $0.01 \mu \mathrm{M}$ & $0.1 \mu \mathrm{M}$ & $1 \mu \mathrm{M}$ & $10 \mu \mathrm{M}$ \\
\hline & \multirow[t]{6}{*}{$8-10(2)$} & $1.0 \mathrm{~Hz}$ & $\mathrm{APD}_{90}(\mathrm{~ms})$ & $369 \pm 16$ & $-1 \pm 1 \%$ & $5 \pm 2 \%{ }^{\#}$ & $-34 \pm 6 \%{ }^{\#}$ & $-74 \pm 2 \%{ }^{\#}$ \\
\hline & & & $\begin{array}{c}\operatorname{STV}\left(\mathrm{APD}_{90}\right) \\
(\mathrm{ms})\end{array}$ & $8 \pm 1$ & $8 \pm 1$ & $8 \pm 1$ & $10 \pm 2$ & $9 \pm 5$ \\
\hline & & & Triangulation & $1.15 \pm 0.01$ & $1.15 \pm 0.01$ & $1.17 \pm 0.02$ & $1.27 \pm 0.04 *$ & $1.36 \pm 0.05^{\#}$ \\
\hline & & $0.5 \mathrm{~Hz}$ & $\mathrm{APD}_{90}(\mathrm{~ms})$ & $424 \pm 25$ & $-0.7 \pm 1 \%$ & $6 \pm 2 \%{ }^{\#}$ & $-17 \pm 6 \% * \$$ & $-67 \pm 3 \%{ }^{\#}$ \\
\hline & & & $\begin{array}{c}\operatorname{STV}\left(A^{A P D} D_{90}\right) \\
(\mathrm{ms})\end{array}$ & $10 \pm 2$ & $15 \pm 5$ & $15 \pm 5$ & $6 \pm 2$ & $3 \pm 1^{\#}$ \\
\hline & & & Triangulation & $1.15 \pm 0.01$ & $1.15 \pm 0.01$ & $1.15 \pm 0.01$ & $1.20 \pm 0.04$ & $1.36 \pm 0.04^{\#}$ \\
\hline \multirow[t]{7}{*}{ Pinacidil } & \multirow{7}{*}{ 3-7 (1) } & & & & $1 \mu \mathrm{M}$ & $3 \mu \mathrm{M}$ & $10 \mu \mathrm{M}$ & $30 \mu \mathrm{M}$ \\
\hline & & $1.0 \mathrm{~Hz}$ & $\mathrm{APD}_{90}(\mathrm{~ms})$ & $397 \pm 18$ & $-8 \pm 2 \%{ }^{\#}$ & $-9 \pm 2 \% *$ & $-51 \pm 3 \% \#$ & $-86 \pm 1 \%{ }^{\#}$ \\
\hline & & & $\begin{array}{c}\operatorname{STV}\left(\mathrm{APD}_{90}\right) \\
(\mathrm{ms})\end{array}$ & $10 \pm 1$ & $9 \pm 1$ & $8 \pm 1$ & $10 \pm 1$ & $2 \pm 0.2^{\#}$ \\
\hline & & & Triangulation & $1.18 \pm 0.03$ & $1.19 \pm 0.03$ & $1.14 \pm 0.01$ & $1.45 \pm 0.07 *$ & $2.57 \pm 0.25^{*}$ \\
\hline & & $0.5 \mathrm{~Hz}$ & $\mathrm{APD}_{90}(\mathrm{~ms})$ & $457 \pm 37$ & $-10 \pm 2 \% \#$ & $-4 \pm 2 \%$ & $-47 \pm 6 \%{ }^{\#}$ & $-86 \pm 2 \% \#$ \\
\hline & & & $\begin{array}{l}\operatorname{STV}\left(\mathrm{APD}_{90}\right) \\
(\mathrm{ms})\end{array}$ & $22 \pm 8$ & $13 \pm 2$ & $9 \pm 1$ & $8 \pm 1$ & $2 \pm 0.4^{*}$ \\
\hline & & & Triangulation & $1.17 \pm 0.03$ & $1.18 \pm 0.03$ & $1.13 \pm 0.01$ & $1.39 \pm 0.04^{\#}$ & $2.59 \pm 0.29 *$ \\
\hline \multirow[t]{7}{*}{ Diltiazem } & \multirow{7}{*}{$4-5(2)$} & & & & $1 \mu \mathrm{M}$ & $3 \mu \mathrm{M}$ & $10 \mu \mathrm{M}$ & $30 \mu \mathrm{M}$ \\
\hline & & $1.0 \mathrm{~Hz}$ & $\mathrm{APD}_{90}(\mathrm{~ms})$ & $368 \pm 30$ & $-4 \pm 1 \%$ & $-4 \pm 5 \%$ & $-25 \pm 6 \% *$ & $-41 \pm 4 \%{ }^{\#}$ \\
\hline & & & $\begin{array}{c}\operatorname{STV}\left(\mathrm{APD}_{90}\right) \\
(\mathrm{ms})\end{array}$ & $5 \pm 1$ & $5 \pm 1$ & $5 \pm 1$ & $7 \pm 2$ & $6 \pm 1$ \\
\hline & & & Triangulation & $1.18 \pm 0.02$ & $1.18 \pm 0.01$ & $1.18 \pm 0.02$ & $1.24 \pm 0.03$ & $1.37 \pm 0.08^{*}$ \\
\hline & & $0.5 \mathrm{~Hz}$ & $\mathrm{APD}_{90}(\mathrm{~ms})$ & $422 \pm 41$ & $-7 \pm 1 \%$ & $-2 \pm 1 \%$ & $-18 \pm 6 \% *$ & $-34 \pm 5 \% \#$ \\
\hline & & & $\begin{array}{l}\operatorname{STV}\left(A^{A P D_{90}}\right) \\
(\mathrm{ms})\end{array}$ & $6 \pm 1$ & $7 \pm 3$ & $5 \pm 1$ & $4 \pm 1$ & $4 \pm 1^{*}$ \\
\hline & & & Triangulation & $1.17 \pm 0.01$ & $1.16 \pm 0.01$ & $1.16 \pm 0.02$ & $1.20 \pm 0.02$ & $1.36 \pm 0.09 *$ \\
\hline
\end{tabular}

Table 2: Effects of reference compounds on STV(APD90) and triangulation (Ratio of APD90/APD50) as a function of change in APD90 in canine LVMMs. Data are expressed as mean \pm SEM. Changes in APD90, STV (APD90) and triangulation were measured in (i) dofetilide-, d-sotalol- and cisapride-treated cells with (before first EAD) and without (once steady state was achieved) EADs and (ii) DL-sotalol-, terfenadine-, pinacidil- and diltiazemtreated cells once steady state was achieved. Differences were tested for statistical significance using the paired (two sample for means; same cardiac preparation) and unpaired (two sample assuming unequal variances; one preparation versus another) Student's t-test. A value of $\mathrm{P}<0.05$ was considered significant. ${ }^{*} \mathrm{P}<0.05$ and $\# \mathrm{P}<0.01$ versus values from vehicle; $\$ \mathrm{P}<0.05$ versus values at $1.0 \mathrm{~Hz}$. Table 2 adapted from Abi-Gerges et al., 2010 with permission from Wiley-Blackwell ${ }^{21}$. 
Table 3

\begin{tabular}{|c|c|c|}
\hline Problem & Possible cause & Solution \\
\hline \multirow[t]{9}{*}{$\begin{array}{l}\text { Cell does not respond to the voltage } \\
\text { stimulus or stops visibly contracting when } \\
\text { di-4-ANEPPS-based method is employed } \\
\text { for measuring the AP }\end{array}$} & $\begin{array}{l}\text { The magnitude of the } \\
\text { voltage-stimulus below the } \\
\text { excitability threshold of the } \\
\text { LVMM cell }\end{array}$ & $\begin{array}{l}\text { Increase the magnitude } \\
\text { (amplitude and/or duration) } \\
\text { of the voltage- stimulus }\end{array}$ \\
\hline & $\begin{array}{l}\text { During perfusion with a } \\
\text { compound }\end{array}$ & $\begin{array}{l}\text { Increase the magnitude } \\
\text { (amplitude and/or duration) } \\
\text { of the voltage- stimulus }\end{array}$ \\
\hline & Break in stimulating electrode & $\begin{array}{l}\text { Re-solder the stimulation } \\
\text { electrode }\end{array}$ \\
\hline & $\begin{array}{l}\text { HSE stimulator faulty } \\
\text { Temperature of the FHD } \\
\text { microscope chamber system } \\
\text { not adequate }\end{array}$ & $\begin{array}{l}\text { Replace the stimulator } \\
\text { Verify temperature and } \\
\text { adjust it if necessary }\end{array}$ \\
\hline & & $\begin{array}{l}\text { Check the vacuum pump and } \\
\text { clean the outflow }\end{array}$ \\
\hline & & $\begin{array}{l}\text { If broken, remove Corning } \\
\text { Cover Glass, clean the oil } \\
\text { objective / re-immersed with } \\
\text { oil for fluorescence and put a } \\
\text { new cover glass }\end{array}$ \\
\hline & $\begin{array}{l}\text { Cell MicroControls mTCII } \\
\text { temperature controller \& } \\
\text { Heater faulty }\end{array}$ & $\begin{array}{l}\text { Replace the controller \& } \\
\text { heater }\end{array}$ \\
\hline & AP morphology is not optimal & Try a new LVMM cell \\
\hline & LVMM cell not healthy & Try a new LVMM cell \\
\hline \multirow[t]{2}{*}{$\begin{array}{l}\text { Cell contracts and looses striations upon } \\
\text { contact with the microelectrode }\end{array}$} & $\begin{array}{l}\text { Microelecrode itself is not a } \\
\text { good shape }\end{array}$ & $\begin{array}{l}\text { Check the resistance of the } \\
\text { microelectrode before } \\
\text { touching cell and if necessary } \\
\text { adjust microelectrode } \\
\text { fabrication settings }\end{array}$ \\
\hline & $\begin{array}{l}\text { Cell contact was made too } \\
\text { quickly }\end{array}$ & Try a new LVMM cell \\
\hline
\end{tabular}




\begin{tabular}{|c|c|c|}
\hline Problem & Possible cause & Solution \\
\hline \multirow[t]{3}{*}{ Recordings show electrical instability } & $\begin{array}{l}\text { Earthing of setup is not } \\
\text { optimal }\end{array}$ & $\begin{array}{l}\text { Check earthing of set-up and } \\
\text { change if necessary }\end{array}$ \\
\hline & Air bubbles in microelectrode & $\begin{array}{l}\text { Ensure the microelectrode is } \\
\text { free of air bubble before } \\
\text { placing into electrode holder }\end{array}$ \\
\hline & $\begin{array}{l}\text { Grounding electrode is not in } \\
\text { solution }\end{array}$ & $\begin{array}{l}\text { Ensure the grounding } \\
\text { electrode is covered by } \\
\text { solution in the bath }\end{array}$ \\
\hline \multirow[t]{3}{*}{$\begin{array}{l}\text { AP morphology is not optimal or there is } \\
\text { no AP on stimulation }\end{array}$} & $\begin{array}{l}\text { The magnitude of the } \\
\text { voltage-stimulus is below the } \\
\text { excitability threshold of the } \\
\text { LVMM cell }\end{array}$ & $\begin{array}{l}\text { Increase the magnitude } \\
\text { (amplitude and/or duration) } \\
\text { of the voltage- stimulus }\end{array}$ \\
\hline & $\begin{array}{l}\text { The temperature of the bath } \\
\text { solution is not at } 37^{\circ} \mathrm{C}\end{array}$ & $\begin{array}{l}\text { Verify temperature and } \\
\text { adjust it if necessary }\end{array}$ \\
\hline & $\begin{array}{l}\text { Perfusion is not at an optimal } \\
\text { rate }\end{array}$ & $\begin{array}{l}\text { Verify flow of perfusate is } \\
\text { running correctly and adjust } \\
\text { if appropriate }\end{array}$ \\
\hline
\end{tabular}

Table 3: Troubleshooting guide for the recording of APs in the isolated canine LVMMs.

\subsubsection{Anticipated Results}

Figure 4 illustrates results of a Basic Protocol study evaluating the electrophysiological effects of reference compounds on the AP of isolated canine LVMMs. The reference compounds selected for the optical APD validation study consisted of three selective $\mathrm{I}_{\mathrm{Kr}}$ blockers (dofetilide, D-sotalol, and E-4031), three multiple ion channel blockers (terfenadine, cisapride, and alfuzosin; $\mathrm{I}_{\mathrm{Kr}}$ inhibitors with additional actions on other cardiac ion channels), an agonist of the late $\mathrm{I}_{\mathrm{Na}}$ (anemone toxin II [ATX-II]), an opener of the ATP sensitive cardiac $\mathrm{K}^{+}$current, $\mathrm{I}_{\text {KATP }}$ (pinacidil), and an inhibitor of the L-type calcium current, $\mathrm{I}_{\text {CaL }}$ (diltiazem). APD data obtained with these nine compounds were as expected, except (1) D-sotalol-induced increases in duration were smaller than those caused by other class III antiarrhythmics, and (2) increases in APD were not detected using low concentrations of terfenadine.To investigate whether a test compound has a proarrhythmic potential, the procedure described in Alternate Protocol 1 should be employed. APD data obtained with dofetilide, D-sotalol, cisapride, terfenadine, pinacidil, and diltiazem were as expected (Table 2). Additionally, LVMMs exhibited a proarrhythmic response to $\mathrm{I}_{\mathrm{Kr}}$ blockers and ATX-II (Table 2 and Figure 6). Incidence of EADs was not related to 
differences in AP prolongation, but corresponded to BVR, quantified as STV (APD) (Table 2 and Figure 6. ${ }^{7,21}$ ). Moreover, assessment of changes in the AP shape (e.g., triangulation ${ }^{19}$ ), in addition to changes in APD and STV (APD), may also be helpful for assessing the arrythmogenic risk of a test compound (Table 2). Blockade of the $\mathrm{I}_{\mathrm{Ks}}$ current with HMR1556 caused very little change in APD when compared to baseline conditions (Figure 6), but predisposed the myocyte to increased BVR during $\beta A R$ stimulation, especially at fast rates and during the window of DAD occurrence (Figure 7). DADs were generated in all cells and EADs in most cells (Figure $7^{7}$ ). In summary, LVMMs provide a suitable preclinical model to frontload the effects of new drugs on optically measured APD and also yield information about putative indicators of proarrhythmia (BVR, EADs, and DADs) that add value to an integrated QT/TdP risk assessment before a compound enters preclinical development.

\subsubsection{Time Considerations}

For the Basic Protocol, $30 \mathrm{~min}$ are needed to prepare LVMM cells (Figure 1), followed by $1 \mathrm{hr}$ of stabilization at room temperature in normal Tyrode's solution containing $1.8 \mathrm{mM} \mathrm{CaCl}_{2}$. The stabilization period can be used to prepare the optical experimental setup and test compound solutions. Thereafter, $\sim 30$ min are needed to load the cells with di-4-ANEPPS. Performing one experiment evaluating the effects of a test compound at four ascending concentrations on the AP of an isolated LVMM cell requires $30 \mathrm{~min}$. To conduct a study, an additional 2-3 hr are required for data acquisition and to take recordings from four additional LVMM cells, and 10 min are needed for additional off-line analysis (including statistics). Cleaning between experiments takes $5 \mathrm{~min}$, with the loading of new cells beginning $10 \mathrm{~min}$ prior to the completion of the experiment in progress. If the LVMM does not respond to field stimulation, or if the recorded AP is not valid for initiation of the experimental protocol, a new cell can immediately be selected from the FHD microscope chamber system (Figure 2, bottom left) without the need for a new stabilization period. Some 20 min are needed at the end of the experimental day to thoroughly clean the FHD microscope chamber system and superfusion tubing (Figure 2, top right and bottom left). To perform one of the experiments detailed in Alternate Protocols 1 and 2, 30$60 \mathrm{~min}$ are required after the myocytes have been isolated and stabilized, although obtaining a healthy cell with acceptable AP characteristics can take anywhere from a few minutes to a few hours. Around 10 min are needed at the end of the day for thorough cleaning of the perfusion system and bath. In general, cells can be used for recording for up to $36 \mathrm{hr}$ after isolation. 


\section{References}

(1) Anon (2005a). The nonclinical evaluation of the potential for delayed ventricular repolarization (QT interval prolongation) by human pharmaceuticals. ICH S7B CHMP/ICH/423/02, 25-5-2005.

(2) Anon (2005b). The clinical evaluation of QT/QTc interval prolongation and proarrhythmic potential for non-antiarrhythmic drugs. ICH E14 CHMP/ICH/2/04, 25-5-2005.

(3) Hardy, M.E.L., Pollard, C.E., Small, B.G., Bridgland-Taylor, M., Woods, A.J., Valentin, J-P., and Abi-Gerges, N. Validation of a voltage-sensitive dye (di-4ANEPPS)-based method for assessing drug-induced delayed repolarisation in Beagle dog left ventricular midmyocardial myocytes. J. Pharmacol. Toxicol. Methods. 2009;60:94-106.

(4) Thomsen, M.B., Matz, J., Volders, P.G., and Vos, M.A. 2006. Assessing the proarrhythmic potential of drugs: current status of models and surrogate parameters of torsades de pointes arrhythmias. Pharmacol. Ther. 2006;112:150-170.

(5) Antzelevitch, C. Heterogeneity and cardiac arrhythmias: An overview. Heart Rhythm. 2007;4:964-972.

(6) Thomsen, M.B., Verduyn, S.C., Stengl, M., Beekman, J.D., De Pater, G., Van Opstal, J., Volders, P.G., and Vos, M.A. Increased short-term variability of repolarization predicts $\mathrm{D}$-sotalol-induced torsades de pointes in dogs. Circulation. 2004;110:2453-2459.

(7) Johnson, D.M., Heijman, J., Pollard, C.E., Valentin, J.P., Crijns, H.J., AbiGerges, N., and Volders, P.G. I $\mathrm{Ks}_{\mathrm{s}}$ restricts excessive beat-to-beat variability of repolarization during beta-adrenergic receptor stimulation. J. Mol. Cell. Cardiol. 2010;48:122-130.

(8) Gallacher DJ, Van de Water A, van der Linde H, Hermans AN, Lu HR, Towart $\mathrm{R}$, Volders PG. In vivo mechanisms precipitating torsades de pointes in a canine model of drug-induced long-QT1 syndrome. CardiovasC Res. 2007;76:247-56.

(9) Abi-Gerges N, Valentin JP, Pollard CE. 2010. Dog left ventricular midmyocardial myocytes for assessment of drug-induced delayed repolarization: short-term variability and proarrhythmic potential. Br J Pharmacol. 2010;159:77-92. 
(10) Fenichel, R.R., Malik, M., Antzelevitch, C., Sanguinetti, M., Roden, D.M., Priori, S.G., Ruskin, J.N., Lipicky, R.J., and Cantilena, L.R. Drug-induced torsades de pointes and implications for drug development. J. Cardiovasc. Electrophysiol. 2004;15:475-495.

(11) Bass, A., Valentin, J.P., Fossa, A.A., and Volders, P.G. Points to consider emerging from a mini-workshop on cardiac safety: Assessing torsades de pointes liability. J. Pharmacol. Toxicol. Methods. 2007;56:91-94.

(12) Lindgren, S., Bass, A.S., Briscoe, R., Bruse, K., Friedrichs, G.S., Kallman, M.J., Markgraf, C., Patmore, L., and Pugsley, M.K. Benchmarking safety pharmacology regulatory packages and best practice. J. Pharmacol. Toxicol. Methods. 2008;58:99-109.

(13) Szabo, G., Szentandrassy, N., Biro, T., Toth, B.I., Czifra, G., Magyar, J., Bányász, T., Varró, A., Kovács, L., and Nánási, P.P. Asymmetrical distribution of ion channels in canine and human left-ventricularwall: Epicardium versus midmyocardium. Pflugers Arch. 2005;450:307-316.

(14) Gralinski, M.R. The dog's role in the preclinical assessment of QT interval prolongation. Toxicol. Pathol. 2003;31(Suppl.):11-16.

(15) Schroeder, K., Neagle, B., Trezise, D.J., and Worley, J. IonworksHT: A new highthroughput electrophysiology measurement platform. J. Biomol. Screen. 2003;8:50-64.

(16) Bridgland-Taylor, M.H., Hargreaves, A.C., Easter, A., Orme, A., Henthorn, D.C., Ding, M.,Davis, A.M., Small, B.G., Heapy, C.G., Abi-Gerges, N., Persson, F., Jacobson, I., Sullivan, M., Albertson, N., Hammond, T.G., Sullivan, E., Valentin, J.P., and Pollard, C.E. Optimisation and validation of a mediumthroughput electrophysiology-based hERG assay using IonWorks HT. J. Pharmacol. Toxicol. Methods. 2006;54:189-199.

(17) Harmer, A.R., Abi-Gerges, N., Easter, A., Woods, A., Lawrence, C.L., Small, B.G., Valentin, J.P., and Pollard, C.E. Optimisation and validation of a medium-throughput electrophysiology based hNav1.5 assay using IonWorks $^{\mathrm{TM}}$. J. Pharmacol. Toxicol. Methods. 2008;57:30-41.

(18) Volders PG, Stengl M, van Opstal JM, Gerlach U, Spätjens RL, Beekman JD, Sipido $K R$, Vos MA. Probing the contribution of $\mathrm{I}_{\mathrm{Ks}}$ to canine ventricular repolarization: key role for beta-adrenergic receptor stimulation.Circulation. 2003; 107:2753-60.

(19) Hondeghem LM. QT prolongation is an unreliable predictor of ventricular arrhythmia. Heart Rhythm. 2008;5:1210-2. 
(20) Jacobson I, Carlsson L, Duker G. Beat-by-beat QT interval variability, but not QT prolongation per se, predicts drug-induced torsades de pointes in the anaesthetised methoxamine-sensitized rabbit. J Pharmacol Toxicol Methods. 2011;63:40-6.

(21) Abi-Gerges, N., Small, B.G., Lawrence, C.L., Hammond,T.G., Valentin, J-P., and Pollard,C.E. Evidence for gender differences in electrophysiological properties of canine Purkinje fibres. Br. J. Pharmacol. 2004;142:1255-1264. 


\section{Chapter 3}

\section{$I_{\text {Ks }}$ Restricts Excessive Beat-to-Beat Variability of Repolarization During $\beta$-Adrenergic Receptor Stimulation}

J Mol Cell Cardiol. 2011;48(1):122-30

Daniel M Johnson • Jordi Heijman • Chris E Pollard • Jean-Pierre Valentin Harry JGM Crijns • Najah Abi-Gerges • Paul GA Volders 


\section{Abstract}

In vivo studies have suggested that increased beat-to-beat variability of ventricular repolarization duration (BVR) is a better predictor of drug-induced torsades de pointes than repolarization prolongation alone. Cellular BVR and its dynamics before proarrhythmic events are poorly understood. We investigated differential responses of BVR in single myocytes during $\mathrm{I}_{\mathrm{Ks}}$ blockade versus $\mathrm{I}_{\mathrm{Kr}}$ blockade and late- $\mathrm{I}_{\mathrm{Na}}$ augmentation, under the influence of $\beta$-adrenergic receptor ( $\beta A R$ ) stimulation. Transmembrane action potentials were recorded from isolated canine left-ventricular midmyocytes at various pacing rates. $\mathrm{I}_{\mathrm{Ks}}$ was blocked by HMR1556, $\mathrm{I}_{\mathrm{Kr}}$ by dofetilide. Late $I_{\mathrm{Na}}$ was augmented by sea anemone toxin-II. Isoproterenol was added for BAR stimulation. BAPTA-AM buffered intracellular $\mathrm{Ca}^{2+}$. SEA0400 partially inhibited the $\mathrm{Na}^{+}-\mathrm{Ca}^{2+}$ exchanger. BVR was quantified as variability of action-potential duration at $90 \%$ repolarization: $\Sigma(\mid A P D 90 ; i+1$ minus APD90; i|)/ [nbeats $\times \sqrt{ } 2$ ] for 30 consecutive action potentials. Baseline BVR was significantly increased by $\mathrm{I}_{\mathrm{Kr}}$ blockade and late- $\mathrm{I}_{\mathrm{Na}}$ augmentation, especially at slow pacing rates. $\beta A R$ stimulation restabilized these BVR changes. In contrast, $\mathrm{I}_{\mathrm{Ks}}$ blockade caused very little change in repolarization when compared to baseline conditions, but predisposed the myocyte to increased BVR during BAR stimulation, especially at fast rates. BAPTA-AM and SEA0400 reduced this excessive BVR and eliminated early afterdepolarizations. In conclusion, $\beta A R$ stimulation exaggerates BVR during $\mathrm{I}_{\mathrm{Ks}}$ blockade, indicating a BVRstabilizing role of $\beta$-adrenergic-sensitive $I_{K s}$. Loss of $I_{K s}$ plus overriding of $\mathrm{Ca}^{2+}$ dependent membrane currents, including inward $\mathrm{Na}^{+}-\mathrm{Ca}^{2+}$-exchange current, conspire to proarrhythmic BVR under these conditions. 


\subsection{I ntroduction}

Beat-to-beat variability of ventricular repolarization duration (BVR) occurs as an apparently random alteration of the repolarization duration (measured from transmembrane or monophasic action potentials (APs), local activation-recovery intervals or QT intervals) in consecutive heart beats at stable rates. Proarrhythmic conditions caused by $\mathrm{I}_{\mathrm{kr}}$ blockade ${ }^{1-3}, \mathrm{I}_{\mathrm{kr}}$-plus- $\mathrm{I}_{\mathrm{Ks}}$ blockade ${ }^{3}$ and late- $\mathrm{I}_{\mathrm{Na}}$ augmentation ${ }^{4}$ are characterized by significant increases of BVR. When increased, BVR is a better predictor of drug-induced torsades de pointes (TdP) than repolarization prolongation alone, at least in canine and rabbit models ${ }^{1-3}$, and in selected human patients ${ }^{5}$.

In the case of selective $I_{K s}$ inhibition in vivo, BVR remains unchanged despite mild QT prolongation ${ }^{3,6}$. However, upon the addition of intense $\beta$-adrenergic receptor $(B A R)$ stimulation pronounced repolarization instability occurs, as evident from amplified BVR, and this precedes the triggering of early afterdepolarizations (EADs), ventricular extrasystoles and TdP ${ }^{6}$. Various antiarrhythmic interventions (e.g., intravenous $\mathrm{KCl}$ administration, $\mathrm{I}_{\mathrm{K}, \mathrm{ATP}}$ activation, steady-state ventricular pacing) that prevent TdP are characterized by stabilization of BVR when often the QT interval is still prolonged ${ }^{7}$. Collectively, these data indicate that a prominent rise of BVR heralds torsadogenic instability of the heart, whereas its decline is an expression of restabilization of electrical activity.

Cellular studies have revealed that the mechanisms underlying BVR reside, at least partly, in the cardiac myocyte. Under baseline conditions at fixed-rate pacing, isolated ventricular myocytes express temporal (i.e., beat-to-beat) variability of the action-potential duration (APD) ${ }^{1,8}$. A direct relationship exists between ratedependent APD and BVR, even during random changes in pacing rate 9 Pharmacological interventions to influence ion channels that operate during the AP plateau can markedly alter baseline BVR. $\mathrm{I}_{\mathrm{kr}}$ blockade ${ }^{1,8,10}$ not only increases the cellular APD, but also BVR, whilst promoting the generation of EADs. Similar effects were noted for late-I $\mathrm{I}_{\mathrm{Na}}$ augmentation with sea anemone toxin-II (ATX-II) ${ }^{11}$. Conversely, the blockade of late- $\mathrm{I}_{\mathrm{Na}}$ with tetrodotoxin ${ }^{8}$ or ranolazine ${ }^{11,12}$ largely suppresses these proarrhythmic repolarization changes. Finally, intracellular $\mathrm{Ca}^{2+}$ chelation with EGTA reduces BVR in myocytes ${ }^{8}$.

In contrast, little is known about the contribution of the potassium current $I_{k s}$ to BVR stability in cardiac myocytes. $\mathrm{I}_{\mathrm{Ks}}$ function is prominent during $\beta A R$ stimulation when it promotes AP shortening by increased protein-kinase-A-dependent activation, and

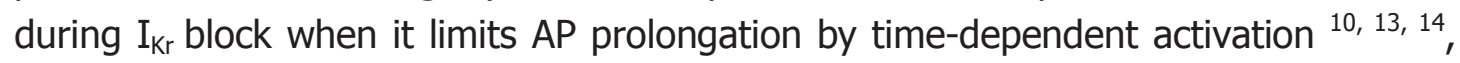
thus providing critical "repolarization reserve" when other repolarizing currents are impaired ${ }^{15,}{ }^{16}$. BVR exaggerates significantly when $I_{\mathrm{KS}}$ is inhibited after AP preprolongation with the $\mathrm{I}_{\mathrm{kr}}$ blocker almokalant ${ }^{10}$. 
In this study we investigated the properties of BVR in single canine left-ventricular (LV) myocytes during superfusion with standard buffer containing physiological concentrations of ions (baseline) and the differential responses of BVR during selective $I_{K s}$ blockade versus $I_{K r}$ blockade and late- $I_{\mathrm{Na}}$ augmentation, in the absence or presence of $\beta A R$ stimulation. Our findings indicate a protective role of $I_{K s}$ in preventing excessive BVR and EAD generation during $\beta A R$ stimulation, and a mechanistic contribution of inward $\mathrm{Na}^{+}-\mathrm{Ca}^{2+}$ exchange to these proarrhythmic sequelae.

\subsection{Materials and Methods}

This investigation conformed with the Guide for the Care and Use of Laboratory Animals published by the US National Institutes of Health (NIH Publication No. 85-23, revised 1996). Animal handling was in accordance with the European Directive for the Protection of Vertebrate Animals Used for Experimental and Other Scientific Purposes (86/609/EU).

\subsubsection{Cell-I solation Procedure}

Twenty three adult female beagle dogs weighing $12.8 \pm 0.3 \mathrm{~kg}$ (range: $10.5-15.0 \mathrm{~kg}$; 9-31 months of age) were used for the myocyte isolations. Anesthesia was induced with $45 \mathrm{mg} / \mathrm{kg}$ pentobarbital. Once full anesthesia was reached, the chest was opened via a left thoracotomy and the heart was excised and placed in an $\mathrm{O}_{2}$-gassed $\mathrm{Ca}^{2+}$-free standard buffer solution at approximately $4^{\circ} \mathrm{C}$. The cell-isolation procedure was the same as previously described ${ }^{17}$. Briefly, the left anterior descending coronary artery was cannulated and perfused. After $\sim 20$ min of collagenase perfusion and subsequent washout of the enzyme, the epicardial surface layer was removed from the LV wedge until a depth of $\geq 3 \mathrm{~mm}$ was reached. Softened tissue samples were collected from the midmyocardial layer underneath while contamination with the endocardium was avoided. Samples were gently agitated, filtered and washed. LV midmyocytes were stored at room temperature in standard buffer solution and only quiescent rod-shaped cells with clear cross-striations were used for the experiments.

\subsubsection{Cellular Recordings}

Transmembrane APs were recorded at $37^{\circ} \mathrm{C}$ bath temperature using high-resistance (30-60 M $\Omega$ ) glass microelectrodes filled with $3 \mathrm{M} \mathrm{KCl}$. Intracellular pacing was done at various cycle lengths (CLs) and only cells that showed a spike-and-dome AP morphology were accepted for the experiments. Myocyte contractions were recorded with a video edge motion detector (Crescent Electronics, Sandy, UT, USA). 


\subsubsection{Solutions and Agents}

The standard buffer solution used for the experiments was composed of (in $\mathrm{mM}$ ): $\mathrm{NaCl} 145, \mathrm{KCl} 4.0, \mathrm{CaCl}_{2} 1.8, \mathrm{MgCl} 21.0$, glucose 11 and HEPES 10, pH 7.4 with $\mathrm{NaOH}$ at $37^{\circ} \mathrm{C}$. HMR1556 ((3R,4S)-(+)-N-[3-hydroxy-2,2-dimethyl-6-(4,4,4-trifluorobutoxy)-chroman-4-yl]-N-methylmethanesulfonamide) was used to block $\mathrm{I}_{\mathrm{Ks}}$. At $500 \mathrm{nM}$ it blocks the current completely ${ }^{10}$, while exerting minimal, if any, effects on $\mathrm{I}_{\mathrm{Kr}}$ and other ion currents ${ }^{6,18,19} . \mathrm{I}_{\mathrm{Kr}}$ was selectively blocked by $1 \mu \mathrm{M}$ dofetilide (Apin Chemicals, Abingdon, United Kingdom). Late $\mathrm{I}_{\mathrm{Na}}$ was augmented by $20 \mathrm{nM}$ ATX-II (Sigma, Zwijndrecht, The Netherlands). Intracellular $\mathrm{Ca}^{2+}$ was buffered with $5 \mu \mathrm{M}$ BAPTA-AM (Invitrogen, Breda, The Netherlands). The $\mathrm{Na}^{+}-\mathrm{Ca}^{2+}$ exchanger was inhibited by $300 \mathrm{nM}$ or $1 \mu \mathrm{M}$ SEA0400. At these concentrations SEA0400 has little effect on $\mathrm{I}_{\mathrm{CaL}}$, the cytosolic $\mathrm{Ca}^{2+}$ transient or contraction of canine ventricular myocytes, whereas it blocks $\mathrm{Na}^{+}-\mathrm{Ca}^{2+}$ exchange by $28-80 \%$ (reverse mode > forward mode) depending on cytosolic $\mathrm{Ca}^{2+}$ concentration ${ }^{20,21}$. HMR1556, dofetilide, ATX-II, BAPTA-AM and SEA0400 were initially dissolved in dimethyl sulfoxide and then diluted so that the concentration of the solvent was $<0.1 \%$ in the superfusate, a concentration that has no measurable effects on AP or ionic currents. BAR stimulation was applied as $100 \mathrm{nM}$ isoproterenol (ISO). This agent was originally dissolved in distilled water containing $30 \mu \mathrm{M}$ ascorbic acid and then stored in the dark at $4^{\circ} \mathrm{C}$ until use.

\subsubsection{Data Analysis and Statistics}

BVR was quantified as variability of APD at $90 \%$ repolarization (APD90): $\Sigma$ (|APD90; $i+1$ minus APD90; $i \mid$ )/ [nbeats $\times \sqrt{ } 2$ ] for 30 (or a minimum of 10 ) consecutive APs ${ }^{1}$. In addition, we calculated the coefficient of variability (CV) of APD90 as the percentage of standard deviation/mean APD $90^{8}$.

Quantitative data are expressed as mean \pm SEM. Intergroup comparisons were made with Student's t test for unpaired and paired data groups, after testing for the normality of distribution. Differences were considered statistically significant if $\mathrm{P}<0.05$.

\subsection{Results}

\subsubsection{Baseline Characteristics of BVR in Canine LV Myocytes}

Figure 1A shows a histogram of 300 consecutive APD90s in a representative contracting myocyte during pacing at a $\mathrm{CL}$ of 1000 ms. APD90 was normally distributed about a mean of $276 \mathrm{~ms}$ with a SEM of $0.5 \mathrm{~ms}$. Similar results were obtained from 13 cells. A correlation coefficient of 0.90 was calculated between the histogram and a normal Gaussian fit. This indicated that APD90 variability within the 
canine myocyte occurs randomly, which is consistent with previous data from guinea pig ${ }^{8}$. In 33 cells, pacing rate was varied between CLs of 350 and 4000 ms. APD90 increased on slowing of the pacing rate, as expected ${ }^{22}$. Single representative and pooled APD90 data are shown in Figure 1B.

BVR averaged $5.9 \pm 0.5 \mathrm{~ms}$ at $\mathrm{CL} 1000 \mathrm{~ms}$ (Figure 1B.). Significant increases were calculated at both the fastest and slowest pacing rates; at CL 350 ms BVR was $10.3 \pm 2.3 \mathrm{~ms}$ and at $\mathrm{CL} 4000 \mathrm{~ms} 11.3 \pm 1.3 \mathrm{~ms}$ (both $\mathrm{P}<0.05$ vs CL $1000 \mathrm{~ms}$ ). Similar patterns were found for CV. At CL 1000 ms CV averaged $2.6 \pm 0.2 \%$, increasing to $4.7 \pm 0.8 \%$ and $4.4 \pm 0.5 \%$ at the same extreme rates (both $\mathrm{P}<0.05$ vs. CL $1000 \mathrm{~ms}$; Figure 1B). Thus, at (very) fast pacing, rate-dependent adaptation of APD90 (further decrease) was clearly discordant with that of both BVR and CV (increases). Beat-to-beat alternans of both APD90 and contraction amplitude explained this discordance, as demonstrated in Figure 1C, left panel. APD90 alternans occurred as a very ordered process (interchanging between two values), whereas BVR was apparent as a more random APD90 behavior, generally at slower rates (Figure 1C, right panel; same cell). This is further exemplified in the Poincaré plots of Figure 1D. These data suggest distinct mechanisms for BVR and APD90 alternans. Both BVR and CV expressed similar patterns of rate-dependent repolarization variability. As BVR incorporates the beat-to-beat consecutiveness of APD90 in its formulation whereas CV does not, we used BVR in the remaining analyses. 
A

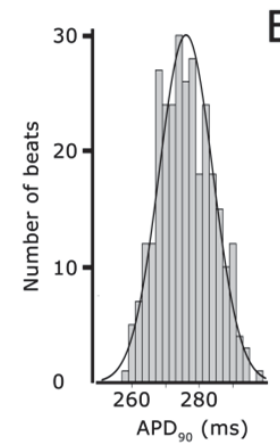

B

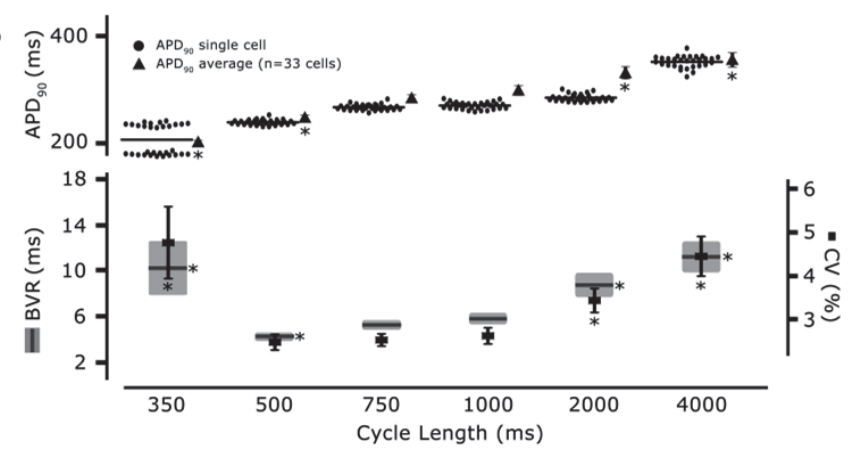

C
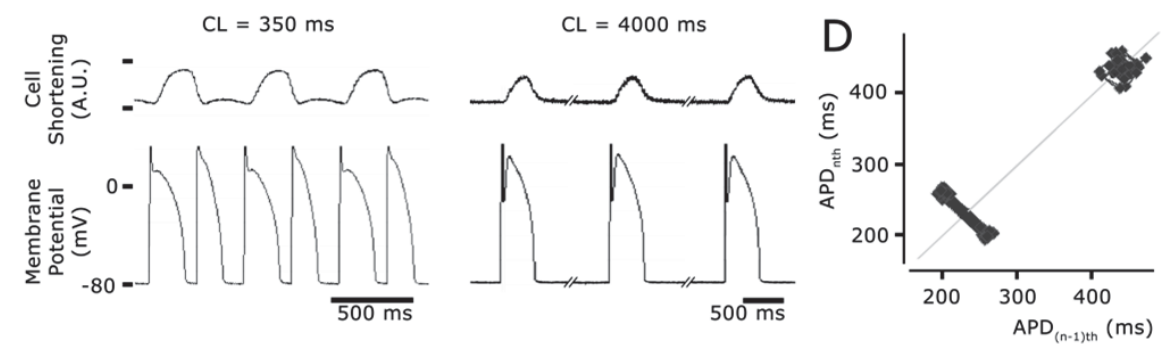

Figure 1: Baseline characteristics of BVR in canine LV myocytes.

A. Histogram of APD90 from 300 consecutive cycles in a representative cell with Gaussian fit (solid line) to the histogram data. Bins of 2 ms width. B. Single-cell (30 APs) and average APD90 as a function of pacing CL (Top panel). Bottom panel illustrates the $C L$ dependence of both BVR and CV. Average parameters are shown \pm SEM. C. Representative AP and contraction recordings from the same cell paced at $350 \mathrm{~ms}$ and $4000 \mathrm{~ms} \mathrm{CL}$. D. Poincaré plots of APD90 from the cell shown in C. at the CL of $350 \mathrm{~ms}$ (left) and $4000 \mathrm{~ms}$ (right). Of note is the repetitive behavior seen at $350 \mathrm{~ms}$, whereas at $4000 \mathrm{~ms}$ the recordings are more chaotic. APD represents APD90. CV, coefficient of variability. CL, cycle length. A.U., arbitrary units.

\subsubsection{Influences of Hyper- and Hypokalemia on BVR}

Next, we examined the influences of hyper- and hypokalemia. Representative results are shown in Figure 2. Myocytes $(n=4)$ were constantly paced at CL 2000 ms. For hyperkalemia, $\left[\mathrm{K}^{+}\right]_{0}$ was raised to $7.0 \mathrm{mM}$ in the superfusate. Along with APD90 shortening $(-15 \%$ from $264 \pm 35 \mathrm{~ms})$, BVR decreased from $5.3 \pm 1.2 \mathrm{~ms}$ (normokalemia) to $3.0 \pm 0.3 \mathrm{~ms}(\mathrm{P}<0.05)$. For hypokalemia, $\left[\mathrm{K}^{+}\right]_{\mathrm{o}}$ was lowered to 2.5 $\mathrm{mM}$. Now, APD90 prolonged (+26\%) and BVR increased to $9.0 \pm 0.8 \mathrm{~ms}(\mathrm{P}<0.05)$. No EADs were observed. Resting membrane potentials varied with the $\left[\mathrm{K}^{+}\right]_{\mathrm{o}}$ changes, as expected (see Figure 2A). 


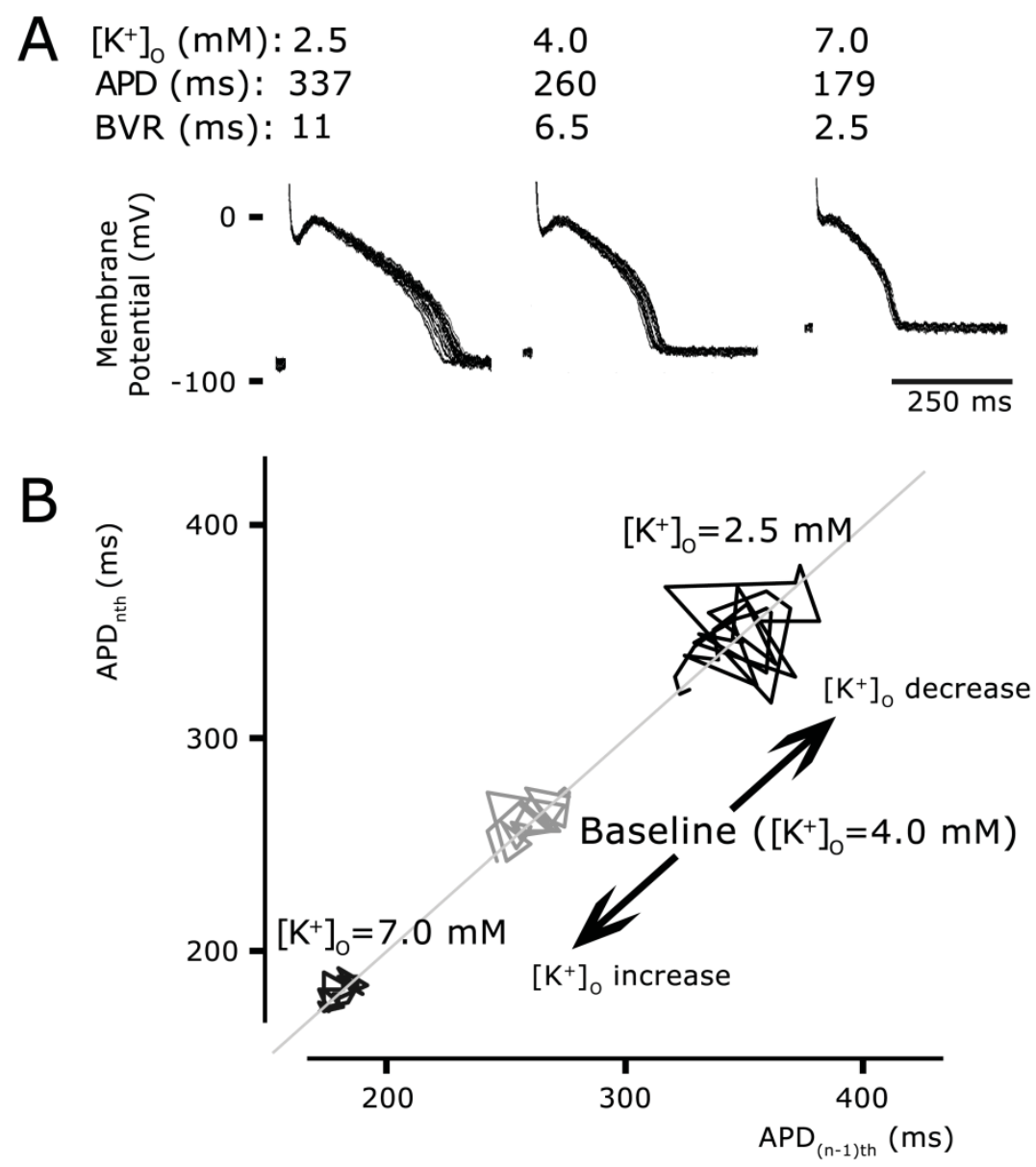

Figure 2: Response of APD and BVR to changes in $\left[\mathrm{K}^{+}\right]_{0}$.

A. APs recorded from 30 consecutive cycles in a single canine LV myocyte paced at a cycle length of $2000 \mathrm{~ms}$ under various $\left[\mathrm{K}^{+}\right]_{\mathrm{O}}$. BVR and APD values and $\left[\mathrm{K}^{+}\right]_{0}$ are indicated above. B. Poincaré plots of APD90 of each of these traces are shown. An increase in BVR is seen during a state of hypokalemia, and a decrease in a state of hyperkalemia.

\subsubsection{BVR Instability and EAD Generation during $I_{\mathrm{Kr}}$ Blockade or late- $\mathrm{I}_{\mathrm{Na}}$ Augmentation, and Rescue by $\beta A R$ Stimulation}

$\mathrm{I}_{\mathrm{Kr}}$ blockade with dofetilide $(1 \mu \mathrm{M})$ and late- $\mathrm{I}_{\mathrm{Na}}$ augmentation with ATX-II (20 nM) increased APD90 and BVR at pacing CLs of 500, 1000 and 2000 ms, but most significantly at the slower rate. This is shown in Figure 3. In the case of dofetilide and for CL $1000 \mathrm{~ms}$, BVR changed from $5.6 \pm 0.5 \mathrm{~ms}$ at baseline to $16.1 \pm 2.9 \mathrm{~ms}$ $(P<0.05 ; 19$ cells; APD90 $+37 \%$ from $297 \pm 15 \mathrm{~ms})$, as calculated from APD90s before the first occurrence of EADs (if present; see below). Similarly, in the case of ATX-II, BVR increased from $4.4 \pm 0.5 \mathrm{~ms}$ to $15.5 \pm 1.8 \mathrm{~ms}(P<0.05 ; 12$ cells; APD90 $+47 \%$ from $255 \pm 17 \mathrm{~ms}$ ). APD90 histograms remained normally distributed during both dofetilide and ATX-II (data not shown). 

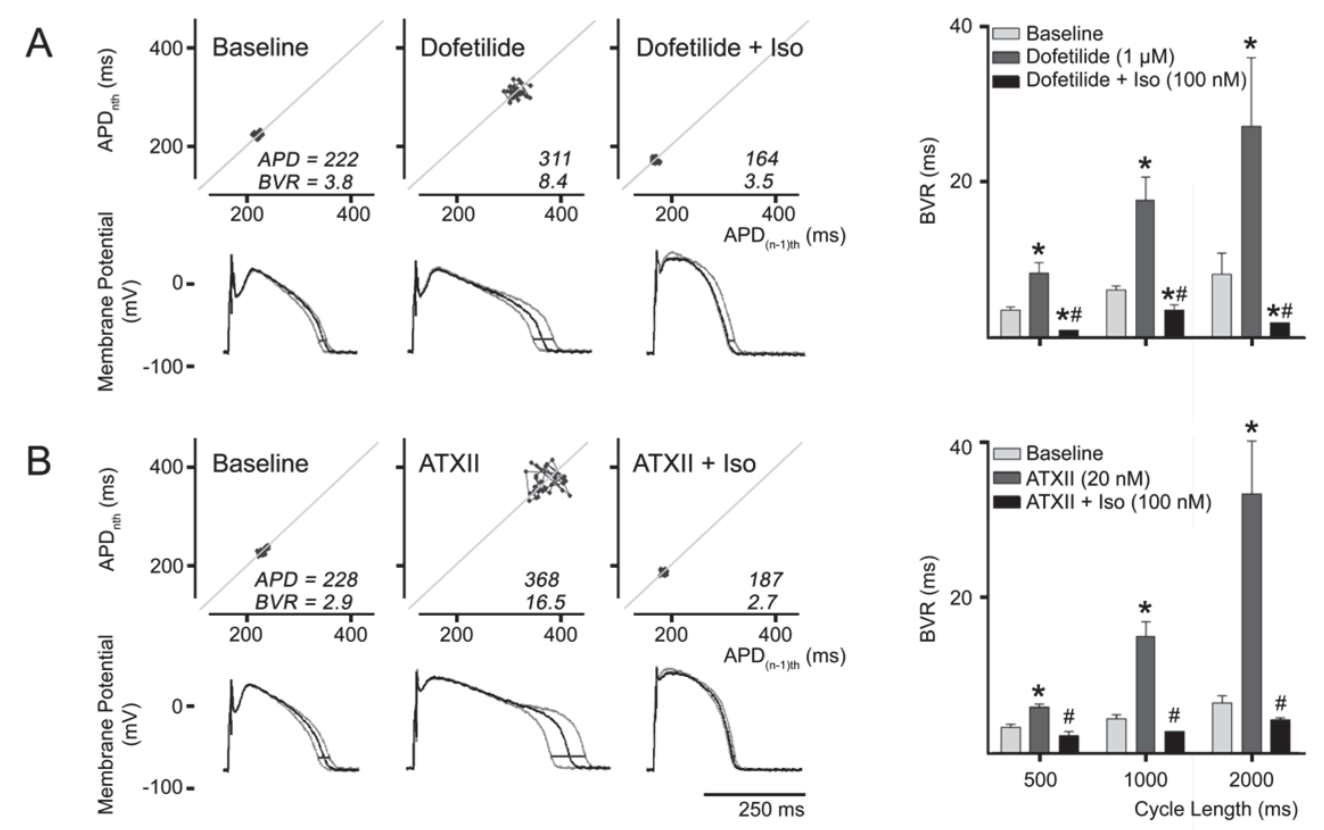

Figure 3: Increases in repolarization instability caused by both $I_{\mathrm{kr}}$ blockade and $I_{\mathrm{Na}}$ augmentation and rescue by $\beta A R$ stimulation. Representative examples showing the effects of dofetilide $1 \mu \mathrm{M}$ (A.) or ATX-II $20 \mathrm{nM}$ (B.) on BVR at $1000 \mathrm{~ms} \mathrm{CL}$ and rescue by BAR stimulation. AP recordings showing the minimum, maximum and median beats are shown for each condition and the ADP90 and BVR are indicated (left). Poincare plots for each condition are also shown. Group data from all cells is shown on the right. Data is shown as mean + SEM. * $\mathrm{P}<0.05$ versus baseline; \# $\mathrm{P}<0.05$ versus drug challenge.

During these drug challenges, the addition of $\beta A R$ stimulation (ISO; $100 \mathrm{nM}$ ) drastically reduced APD90 and BVR at all cycle lengths, even to values below baseline (Figure 3). EADs were also abolished. For dofetilide at CL $1000 \mathrm{~ms}$, BVR decreased from $16.1 \pm 2.9 \mathrm{~ms}$ to $3.5 \pm 0.7 \mathrm{~ms}$ ( $P<0.05$; APD90 $-102 \%$ to $201 \pm 23$ $\mathrm{ms}$ ). For ATX-II at the same CL, BVR was reduced from $15.5 \pm 1.8 \mathrm{~ms}$ to $2.8 \pm 0.3 \mathrm{~ms}$ (P<0.05; APD90 $-40 \%$ to $180 \pm 10 \mathrm{~ms}$ ). In some cells, delayed afterdepolarizations (DADs) appeared during $\beta A R$ stimulation and drug challenge, however these did not reach the threshold for triggering APs.

\subsubsection{BVR Instability and EAD Generation by $\beta A R$ Stimulation during $I_{\mathrm{Ks}}$ Blockade}

$\mathrm{I}_{\mathrm{Ks}}$ blockade with HMR1556 (500 nM) did not affect APD90 at pacing CLs of 500, 1000 and $2000 \mathrm{~ms}$, in line with previous results ${ }^{10,}{ }^{23}$. For example, at CL $1000 \mathrm{~ms}$ APD90 was $249 \pm 8 \mathrm{~ms}$ at baseline compared to $250 \pm 8 \mathrm{~ms}$ under HMR1556. Likewise, BVR remained unaltered at CLs of 500 and 1000 ms. However at CL 2000 ms, it increased moderately but significantly (Figure 4, bar graph; $n=16$ ). APD90 histograms remained normally distributed during HMR1556. No EADs or DADs were observed during HMR1556 only. 

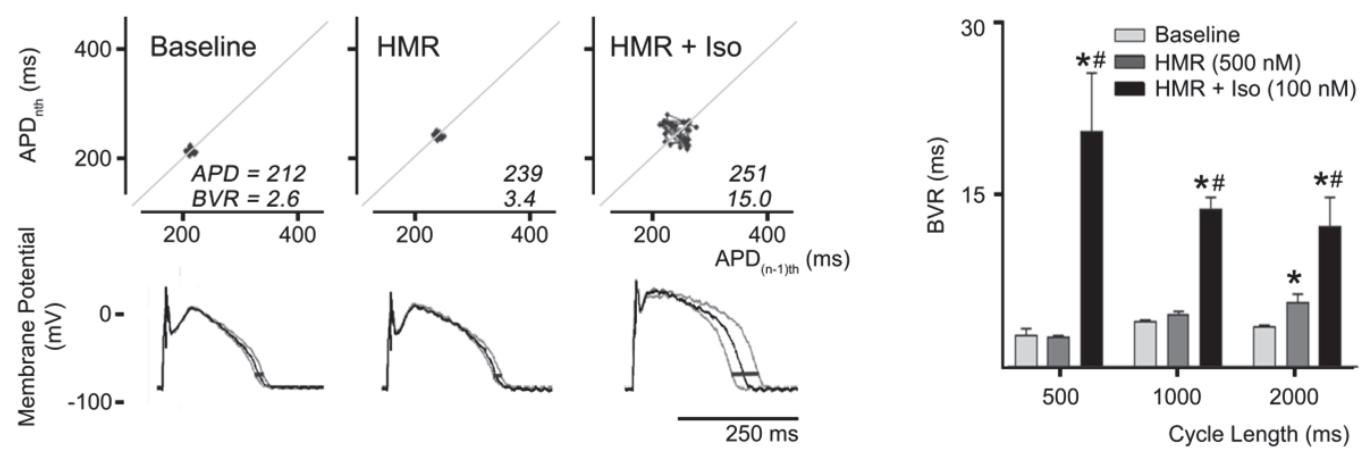

Figure 4: $\beta A R$ stimulation unmasks repolarization instability caused by $\mathrm{I}_{\mathrm{ks}}$ blockade.

Representative traces showing the minimum, maximum and median action potential under baseline conditions, $\mathrm{I}_{\mathrm{Ks}}$ blockade and $\mathrm{I}_{\mathrm{Ks}}$ blockade plus $\beta A R$ stimulation during pacing at 1000 ms. Poincaré plots, APD90 and BVR values for the particular cell are also shown (Left Panel). Group data from all cells are shown in the Right Panel showing mean+SEM.

* $\mathrm{P}<0.05$ versus baseline; \# $\mathrm{P}<0.05$ versus drug challenge.

The addition of $\beta A R$ stimulation during $\mathrm{I}_{\mathrm{Ks}}$ blockade changed this picture drastically: BVR increased at all CLs, but most apparently at CL 500 ms and during the window of DAD occurrence (Figures 4 and 5). DADs were generated in all cells $(n=10)$, and EADs in most of them. At pacing $\mathrm{CL} 1000 \mathrm{~ms}$, BVR increased to $5.7 \pm 0.3 \mathrm{~ms}$ during HMR1556 plus ISO before the occurrence of DADs from a value of $4.2 \pm 0.2$ under HMR1556 alone. During the subsequent phase with DADs, BVR increased further to $14.8 \pm 1.5 \mathrm{~ms}(\mathrm{P}<0.05)$. APD90 also showed a significant increase at all CLs. For example at CL 1000 ms, APD90 averaged $291 \pm 15$ ms (+17\% versus baseline) during HMR1556 plus ISO in the phase with DADs. Obviously, EADs exaggerated BVR and APD90 even further (Figure 5). BVR and APD90 changes, and the generation of DADs and EADs, were completely reversible upon washout of ISO.

\subsubsection{Differential Magnitudes of BVR in Cells with or without EAD Generation, and Rate Dependence of EADs under Different Conditions}

During $\mathrm{I}_{\mathrm{kr}}$ blockade and late- $\mathrm{I}_{\mathrm{Na}}$ augmentation, BVR increases (most pronounced at slow rates) were significantly larger in myocytes in which EADs ensued, even though BVR was calculated before the first EAD occurred, ( $n=8 / 19$ for dofetilide and $n=5 / 12$ for ATX-II) versus those cells in which no EADs were seen $(n=11 / 19$ and $n=7 / 12$, respectively).

This is shown in Figure 6A (for CL 2000 ms). In contrast, EADs generated during $\mathrm{I}_{\mathrm{Ks}}$ blockade plus $\beta A R$ stimulation were clearly fast-rate dependent (Figure 6B). Under these conditions, BVR was not statistically different for myocytes with ensuing EADs $(n=5 / 10)$ versus those without $(n=5 / 10)$. BAR-stimulated EADs were always preceded by DADs and were always accompanied by early aftercontractions (Figure 7 and Figure 8, middle panels). 

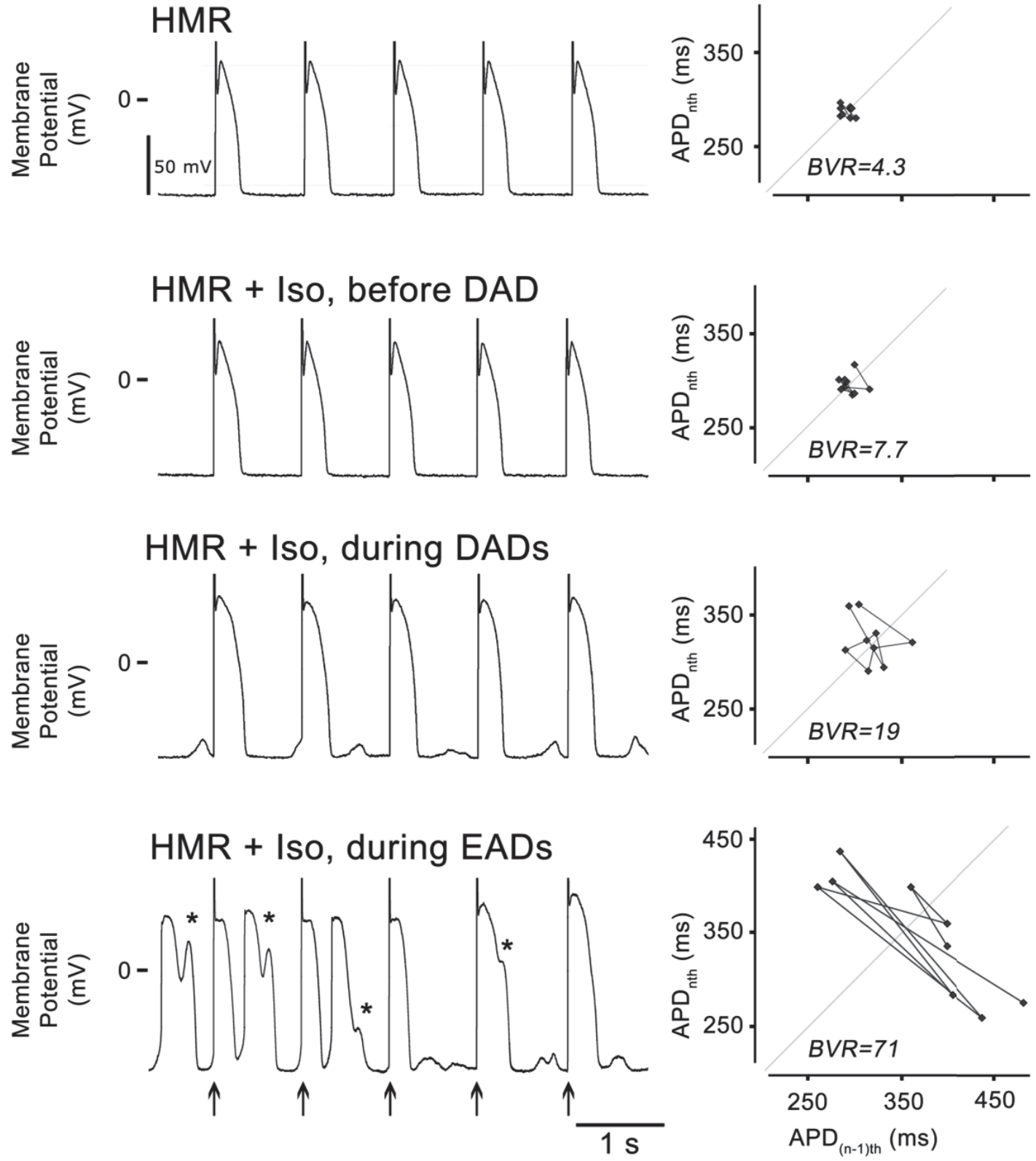

Figure 5: Time course of increased repolarization instability during $I_{K s}$ blockade plus $\beta A R$ stimulation. Upper panel shows consecutively paced APs at $500 \mathrm{~ms} \mathrm{CL}$, together with Poincaré plots during initial perfusion of HMR1556. Addition of $\beta A R$ stimulation increases APD and BVR before DADs are seen (2nd panel from above). The occurrence of DADs (3rd panel) increases APD and BVR further, and DAD triggered APs and EADs are subsequently observed (4th panel). Arrows indicate timings of paced beats, and $*$ indicate EADs. Low amplitude EADs are also generated under these conditions. 
A

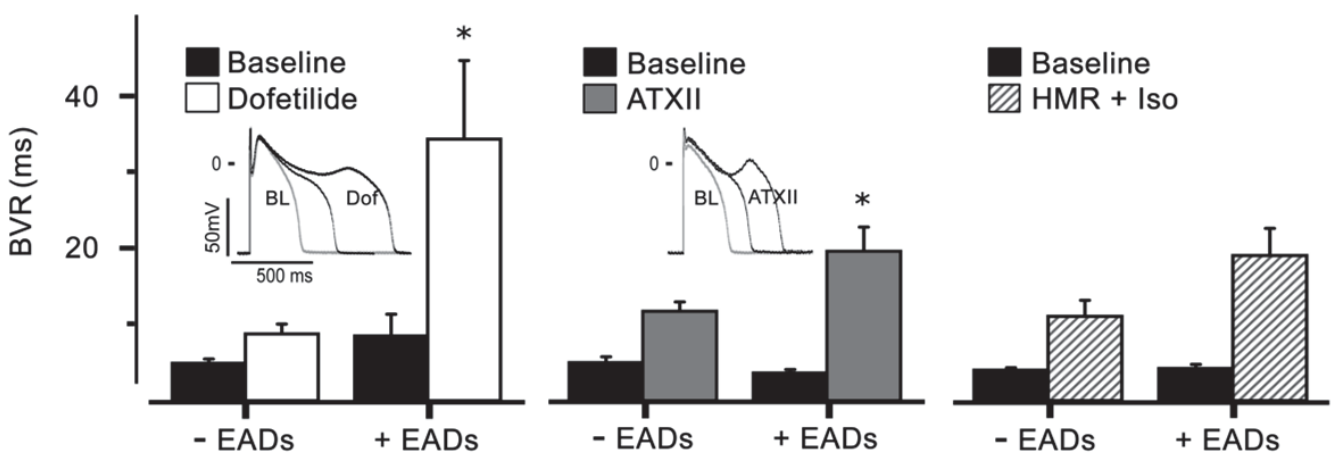

B

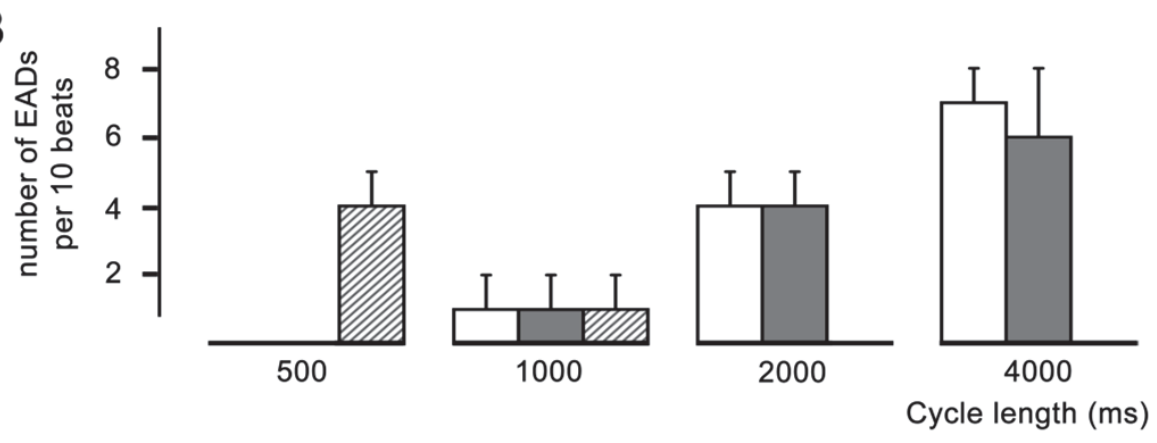

Figure 6: Magnitude of BVR in cells with or without EADs, and rate dependence of EADs under various conditions. A. Bar graph showing BVR values in cells that show EADs compared to those that did not during various drug treatments. Insets show examples of EADs caused by $\mathrm{I}_{\mathrm{Kr}}$ block and $\mathrm{I}_{\mathrm{Na}}$ augmentation together with the preceding beat and the baseline AP. Mean data shown \pm SEM; $B$ L represents baseline. $* P<0.05$ versus $B V R$ in cells with no EADs. B. Bar graph showing the rate dependence of EADs. Number of EADs per 10 beats are plotted at various CLs during all 3 treatment groups. Mean data shown \pm SEM.

Early aftercontractions usually took off before EAD upstrokes (Figure 7, middle panel) and, in fact, occurred often without concrete EADs. Collectively, these data indicated that EADs and DADs during $\mathrm{I}_{\mathrm{Ks}}$ blockade plus $\beta A R$ stimulation are generated as the consequence of cellular $\mathrm{Ca}^{2+}$ overload and spontaneous $\mathrm{Ca}^{2+}$ release from the sarcoplasmic reticulum, and they suggested a mechanistic contribution of inward $\mathrm{Na}^{+}-\mathrm{Ca}^{2+}$ exchange ${ }^{24,25}$. 


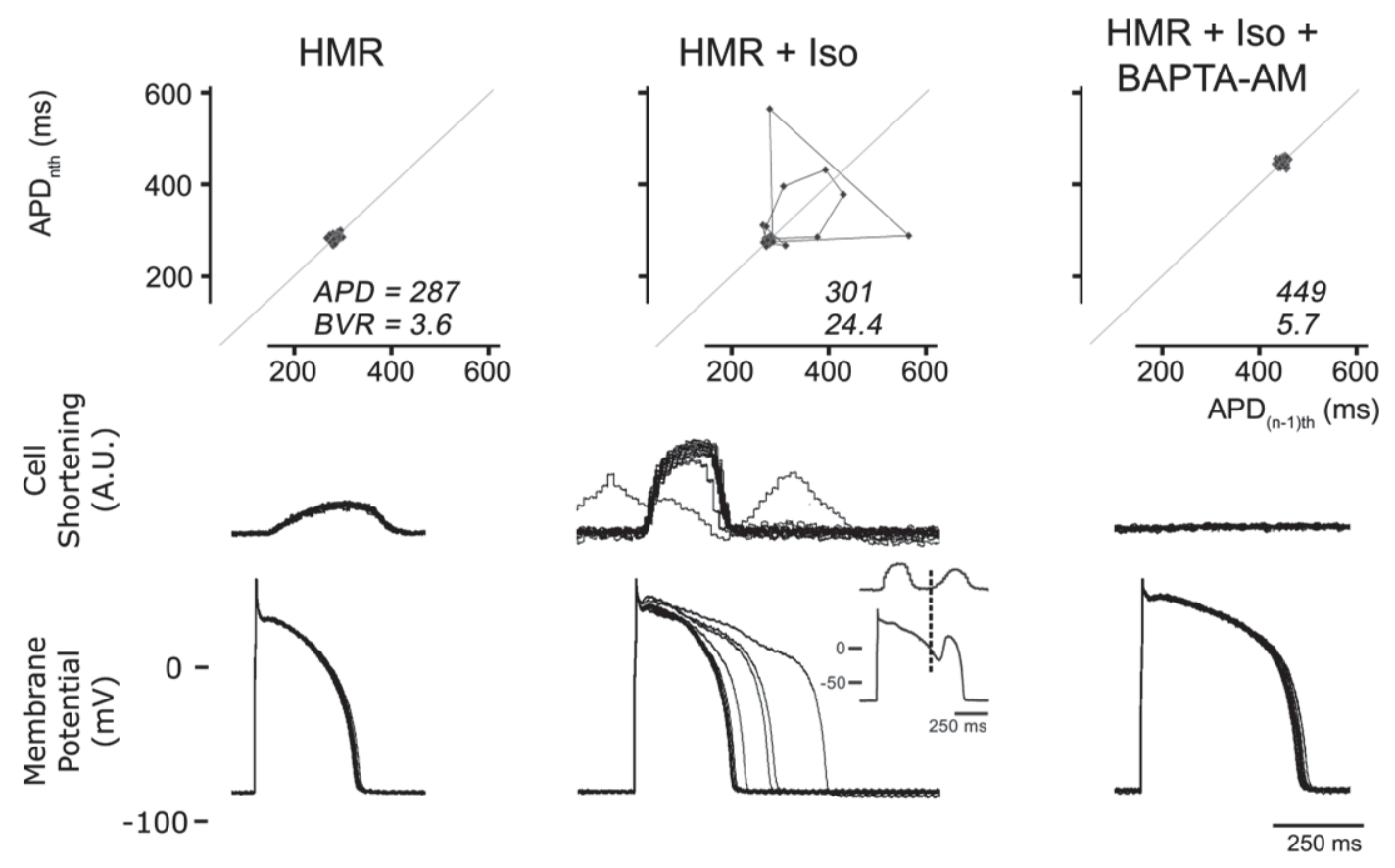

Figure 7: $\mathrm{Ca}^{2+}$ chelator BAPTA-AM rescues from excessive BVR during $\mathrm{I}_{\mathrm{Ks}}$ plus $\beta A R$ stimulation. Representative traces showing 30 consecutive AP and contraction recordings overlapped from the same cell under $I_{K s}$ blockade (left panel), plus $\beta A R$ stimulation (middle panel) and together with $5 \mu \mathrm{M}$ BAPTA-AM (right panel) whilst being paced at $1000 \mathrm{~ms}$. Inset shows an example EAD caused by $\mathrm{I}_{\mathrm{Ks}}$ blockade plus $\beta A R$ stimulation which are eliminated during intracellular calcium buffering despite APD lengthening. Dashed line indicates the initiation of the early aftercontraction which preceded the EAD upstroke. Poincaré plots, APD90 and BVR values for this particular cell are also shown.

\subsubsection{Influences of BAPTA-AM and SEA0400 on BVR Instability and EAD Generation during $I_{\mathrm{Ks}}$ Blockade plus $\beta$ AR stimulation}

As a final series of experiments, we examined the effects of the intracellular $\mathrm{Ca}^{2+}$ chelator BAPTA-AM $(5 \mu \mathrm{M})$ and the $\mathrm{Na}^{+}-\mathrm{Ca}^{2+}$-exchange blocker SEA0400 (300 nM or $1 \mu \mathrm{M})$ on BVR instability and EAD generation by $\beta A R$ stimulation during $\mathrm{I}_{\mathrm{Ks}}$ blockade. Data were obtained in 6 cells. As shown in Figure 7, BAPTA-AM significantly reduced excessive BVR and eliminated all EADs (and DADs). At pacing $\mathrm{CL} 1000 \mathrm{~ms}$, BVR changed from $12.9 \pm 1.6 \mathrm{~ms}$ during HMR1556 plus ISO (before first EADs) to $4.2 \pm 0.1$ ms $(P<0.05)$ during additional BAPTA-AM, even despite extra AP prolongation (APD90 $+56 \%$ ). The latter is likely caused by a reduction in $\mathrm{I}_{\mathrm{CaL}}$ inactivation ${ }^{26}$ and an $\mathrm{I}_{\mathrm{kr}}$-blocking side-effect of BAPTA-AM ${ }^{27}$. BVR restabilization was maximal when all (after) contractile activity was inhibited by the $\mathrm{Ca}^{2+}$ buffer. 

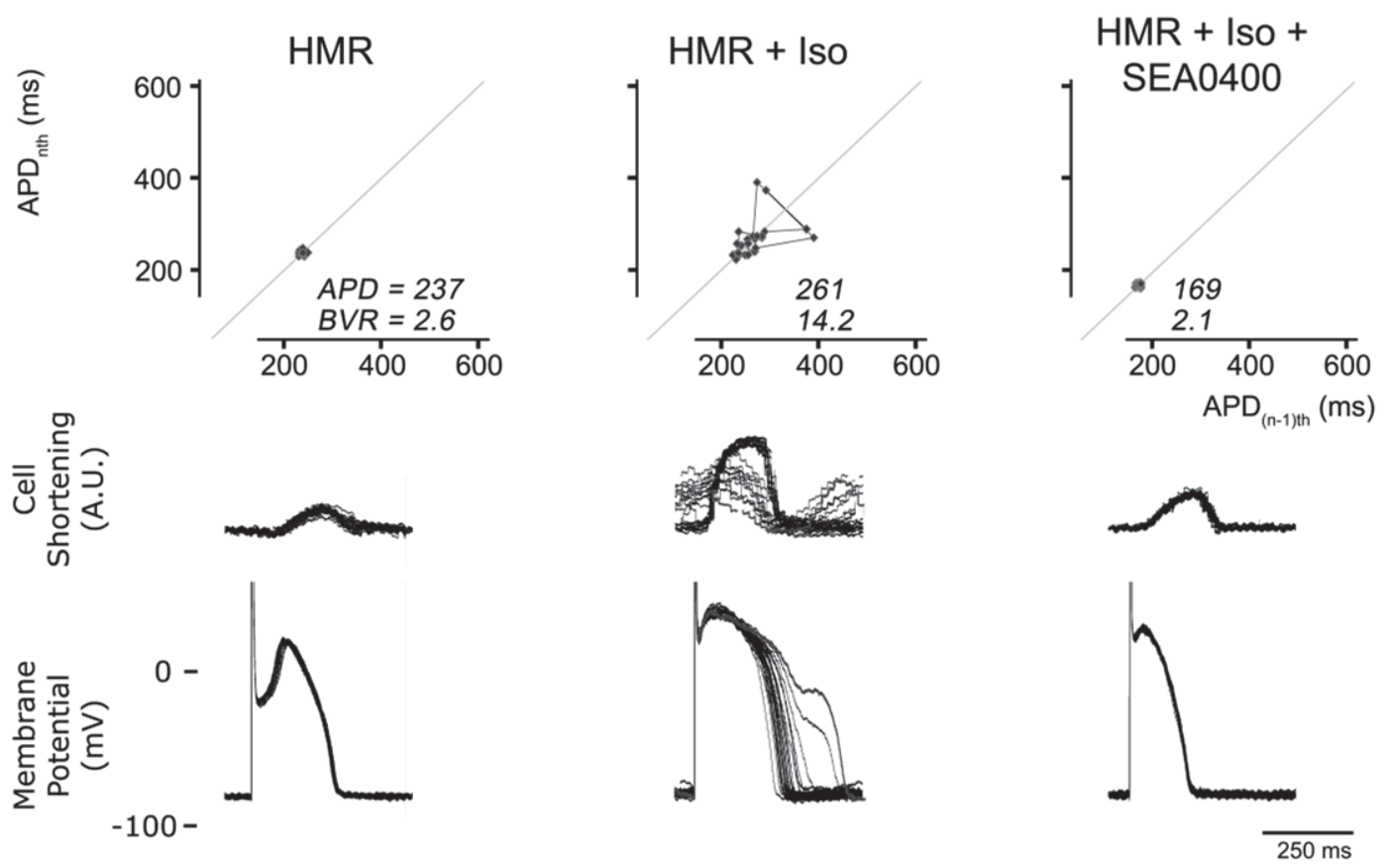

Figure 8: $\mathrm{Na}^{+}-\mathrm{Ca}^{2+}$ exchange inhibitor SEA0400 rescues from excessive BVR during $\mathrm{I}_{\mathrm{Ks}}$ plus $\beta A R$ stimulation. Representative traces showing 30 consecutive AP and contraction recordings from the same cell under $I_{K_{s}}$ blockade (left panel) plus $\beta A R$ stimulation (middle panel) plus $300 \mathrm{nM}$ SEA0400 (right panel) at CL 500 ms. EADs are seen under $\mathrm{I}_{\mathrm{Ks}}$ blockade plus $\beta A R$ stimulation which are eliminated during application of SEA0400. Poincaré plots, APD90 and BVR values for the particular cell are also shown.

Figure 8 illustrates the effects of SEA0400 (300 nM) at CL $500 \mathrm{~ms}$. BVR decreased significantly from $12.2 \pm 1.5 \mathrm{~ms}$ during HMR1556 plus $\beta A R$ stimulation to $2.4 \pm 0.2 \mathrm{~ms}$ $(P<0.05)$ during additional SEA0400. Effects were less pronounced at CLs 1000 and $2000 \mathrm{~ms}$. All EADs were eliminated upon the addition of SEA0400. BVR effects were quantitatively similar for $300 \mathrm{nM}$ and $1 \mu \mathrm{M}$ SEA0400, as were reductions of APD90 and contraction amplitude. At CL $500 \mathrm{~ms}$, APD90 decreased from 246 16 ms during HMR1556 plus $\beta A R$ stimulation to $201 \pm 13 \mathrm{~ms}(\mathrm{P}<0.05)$ during additional SEA0400, whilst contraction amplitudes were decreased by $52 \pm 5 \%$.

\subsection{Discussion}

The mechanisms underlying BVR at the single-cell level are poorly understood. In the present study, we used canine ventricular myocytes to investigate BVR responses at baseline and during various proarrhythmic challenges. Our data indicate that $\mathrm{I}_{\mathrm{KS}}$ stabilizes BVR at slow pacing rates under baseline conditions, despite the absence of changes in APD90. BAR stimulation of $I_{\mathrm{KS}}$ rescues from excessive repolarization instability and EAD generation during $\mathrm{I}_{\mathrm{Kr}}$ block and late- $\mathrm{I}_{\mathrm{Na}}$ augmentation. Furthermore, our results support the notion that $\beta A R$ stimulation during druginduced $\mathrm{I}_{\mathrm{ks}}$ block promotes altered $\mathrm{Ca}^{2+}$ handling and enhanced activation of $\mathrm{Ca}^{2+}$ dependent membrane currents, including inward $\mathrm{Na}^{+}-\mathrm{Ca}^{2+}$ exchange, thus 
exaggerating BVR. Although single myocyte BVR is not the sole contributor to in-vivo QT variability, our novel insights are crucial for the understanding of BVR at the whole organ level such as seen after $\mathrm{I}_{\mathrm{Ks}}$ blockade and $\beta$ AR stimulation ${ }^{6}$.

\subsubsection{Distinction between BVR and Repolarization Alternans}

At baseline, BVR is mainly dependent on APD which in turn is reliant on cycle length. At very fast rates, however, APD90 and BVR become discordant as the result of beat-to-beat repolarization alternans (Figure 1). The latter is an ordered process, characterized by interchanging between two APDs and concomitant contraction amplitudes. Although repolarization alternans is considered arrhythmogenic, EADs are not normally seen under these conditions, at least not in our experiments. Repolarization and contraction alternans arise from beat-to-beat alteration in cellular $\mathrm{Ca}^{2+}$ cycling ${ }^{28}$. BVR, on the other hand, is characterized by an apparently random behavior of APD, generally at intermediate and slow rates. Even during pronounced BVR instability, e.g., by hypokalemia, $\mathrm{I}_{\mathrm{Kr}}$ blockade and late- $\mathrm{I}_{\mathrm{Na}}$ augmentation, contraction amplitudes remain relatively stable (contraction data not shown). Our data thus indicate that in the majority of cases BVR and APD90 show parallel changes. However, in specific conditions they can deviate, for example during $\mathrm{I}_{\mathrm{Ks}}$ blockade plus $\beta A R$ stimulation, and during $I_{\mathrm{Ks}}$ blockade plus $\beta A R$ stimulation and BAPTA-AM. This suggests that BVR is not necessarily dependent on APD90 at all times, which has major mechanistic implications.

Cellular BVR likely results from the stochastic activities of transmembrane ion transporters, including ion channels, during the AP plateau phase. During this phase, around the $0 \mathrm{mV}$ level, the membrane resistance is very high ${ }^{8}$, meaning that minute changes of the ion fluxes across the membrane can significantly affect the membrane potential which in turn will alter the repolarization duration ${ }^{29}$.

Interventions that reduce net outward current will cause a prolongation of this vulnerable phase, meaning that there is an extended period at which the voltage is around the $0 \mathrm{mV}$ level. Stochastic behavior of the ion channels may then increase BVR. It is also possible that this stochastic behavior results indirectly from subsarcolemmal $\mathrm{Ca}^{2+}$ fluctuations that lead to modification of $\mathrm{Ca}^{2+}$-dependent ion currents. Modeling data has shown that during ISO stochastic $\mathrm{I}_{\text {CaL }}$ gating in mode 2 results in APD variability as well as EAD generation ${ }^{30}$, and this could contribute to the BVR seen in this study.

\subsubsection{Cellular BVR during $\beta A R$ stimulation}

In the present study, we have shown that BVR is drastically increased under conditions of $\mathrm{I}_{\mathrm{Kr}}$ blockade or late $\mathrm{I}_{\mathrm{Na}}$ augmentation in isolated canine LV midmyocytes. The addition of $\beta A R$ stimulation, however, causes a major reduction in BVR and eliminates EAD activity (Figure 3). As $I_{\mathrm{Ks}}$ is the main $\beta A$-sensitive outward current in the canine ventricular $A P$, this current appears responsible for the 
restabilization of repolarization. If true, $\beta A R$ stimulation would provoke BVR instability during $\mathrm{I}_{\mathrm{Ks}}$ blockade, as $\mathrm{I}_{\mathrm{Ks}}$ could no longer compensate for the increased $\mathrm{I}_{\mathrm{CaL}}$ caused by $\beta$ AR stimulation, which leads to increased cellular $\mathrm{Ca}^{2+}$ load and APD90. This was indeed the case: BVR increased significantly, despite only moderate increases in APD90 (Figure 4). In this phase, $\beta A R$ stimulation also evoked DADs and EADs (Figure 5). BVR increased after the first generation of DADs (even before EADs occurred; Figure 5), which supports the concept that dynamic subsarcolemmal $\mathrm{Ca}^{2+}$ fluxes during cellular $\mathrm{Ca}^{2+}$ overload and diastolic spontaneous $\mathrm{Ca}^{2+}$ release from the sarcoplasmic reticulum influence the subsequent (paced) APs and BVR via mechanisms yet to be elucidated. Our finding that BVR and EAD generation by $I_{K s}$ blockade and $\beta A R$ stimulation were most pronounced at fast pacing rates (as opposed to $\mathrm{I}_{\mathrm{kr}}$ blockade and late- $\mathrm{I}_{\mathrm{Na}}$ augmentation; Figure 6) fits with this concept. Additional support comes from the result that intracellular $\mathrm{Ca}^{2+}$ chelation with BAPTA-AM reduces beat-to-beat repolarization instability (Figure 7). Moreover, in preliminary experiments in which we used ryanodine $(1 \mu \mathrm{M})$ during $\mathrm{I}_{\mathrm{Ks}}$ blockade and $\beta A R$ stimulation (data not shown), blockade of sarcoplasmic-reticulum $\mathrm{Ca}^{2+}$ release reduced excessive BVR (while inhibiting the generation of afterdepolarizations), again supporting that calcium-dependent membrane currents contribute to BVR.

Our results on afterdepolarization occurrence are partially in agreement with data from the canine LV-wedge preparation ${ }^{31}$. Burashnikov and Antzelevitch ${ }^{31}$ reported that under combined $I_{K s}$ blockade and $\beta A R$ stimulation, DADs were induced in LV epi-, mid- and endocardium. Transmural dispersion of repolarization was accentuated. However, no EAD activity was seen. This apparent discrepancy with our observations in isolated myocytes could be explained by the difference in cell-to-cell electrotonic coupling. The latter, which acts in the LV wedge preparation but not in the isolated cell, has been shown to reduce BVR ${ }^{8}$.

\subsubsection{The $\mathrm{Na}^{+}-\mathrm{Ca}^{2+}$ Exchange I nhibitor SEA0400 Attenuates Excessive BVR and EADs during $I_{\mathrm{Ks}}$ Blockade plus $\beta A R$ stimulation}

Various groups have used the $\mathrm{Na}^{+}-\mathrm{Ca}^{2+}$-exchange inhibitor SEA0400 in attempts to prevent TdP under conditions of $\mathrm{I}_{\mathrm{Kr}}$ block ${ }^{32,33}$ and $\mathrm{I}_{\mathrm{Na}}$ augmentation ${ }^{32}$ with mixed results. Milberg et al. ${ }^{32}$ showed that the application of SEA0400 (1 $\left.\mu \mathrm{M}\right)$ in the presence of sotalol or veratridine resulted in a significant decrease of monophasic APD and an attenuation of EADs in paced Langendorff-perfused rabbit hearts with complete atrioventricular block, supporting the hypothesis that the $\mathrm{Na}^{+}-\mathrm{Ca}^{2+}$ exchanger is an important mediator of EADs ${ }^{25}$. However in the study of Farkas et al. ${ }^{33}$ SEA0400 $(1 \mu \mathrm{M})$ did not suppress dofetilide-induced TdP nor decrease QT variability in unpaced Langendorff-perfused rabbit hearts with complete atrioventricular block. The major difference between these two studies was that in the study of Milberg ${ }^{33}$ hearts were paced at constant cycle lengths, whereas in the alternate study ${ }^{32}$ hearts were allowed to beat at their intrinsic ventricular rate. Upon 
administering SEA0400 in addition to dofetilide, the rate instability increased, and this additional factor could have increased the susceptibility to TdP, at variance with the results of Milberg et al. ${ }^{33}$.

To the best of our knowledge this report is the first to describe the use of $\mathrm{Na}^{+}-\mathrm{Ca}^{2+}-$ exchange inhibition in the presence of $I_{\mathrm{Ks}}$ block combined with $\beta A R$ stimulation (as such mimicking long-QT syndrome type 1 (LQT1)). In line with Milberg ${ }^{33}$, the application of SEA0400 resulted in a significant decrease of transmembrane APD and an attenuation of EADs (Figure 8). Moreover, SEA0400 rescued from excessive BVR, suggesting a repolarization-destabilizing role of inward $\mathrm{Na}^{+}-\mathrm{Ca}^{2+}$-exchange under of $\mathrm{I}_{\mathrm{Ks}}$ blockade combined with $\beta A R$ stimulation ${ }^{24}$.

\subsubsection{Clinical Perspectives}

In LQT1 patients, sympathetic stimulation and epinephrine challenges prolong the QT interval ${ }^{34}$, increase beat-to-beat QT variability ${ }^{35}$ and promote EAD generation ${ }^{34}$. Our present cellular results may thus have important clinical implications, as they support the use of $\mathrm{Ca}^{2+}$ antagonists ${ }^{36}$, $\beta$ AR blockers ${ }^{34,}{ }^{36}$ and even $\mathrm{I}_{\mathrm{K}, \mathrm{ATP}}$ openers ${ }^{34}$ to restrain BVR and reduce the risk of TdP in these patients. Indeed, $\beta A R$ blockade, the cornerstone of pharmacological treatment in LQT1, decreases epinephrine-prolonged monophasic APD and transmural dispersion of repolarization in LQT1 ${ }^{34}$. Likewise,

$\alpha$-adrenergic-receptor blockade with phenylephrine can decrease transmural dispersion of repolarization in LQT1 patients, but causes it to increase in LQT2 patients ${ }^{37}$. Whether the $\mathrm{Na}^{+}-\mathrm{Ca}^{2+}$ exchanger is a suitable target for antiarrhythmic treatment in LQT1 patients is currently under debate.

\subsubsection{Conclusions}

The repolarizing potassium current $\mathrm{I}_{\mathrm{Ks}}$ stabilizes BVR in single canine LV myocytes. During conditions of $\mathrm{I}_{\mathrm{Kr}}$ blockade and late- $\mathrm{I}_{\mathrm{Na}}$ augmentation, $\beta A R$ stimulation of $\mathrm{I}_{\mathrm{Ks}}$ rescues from excessive BVR, AP prolongation and EAD generation. However, during $\mathrm{I}_{\mathrm{Ks}}$ blockade $\beta A R$ stimulation provokes excessive BVR, while promoting the generation of DADs and EADs. The loss of $\mathrm{I}_{\mathrm{Ks}}$ plus overriding of $\mathrm{Ca}^{2+}$-dependent inward currents, including inward $\mathrm{Na}^{+}-\mathrm{Ca}^{2+}$-exchange current, likely conspire to cellular proarrhythmia under these conditions.

\subsection{Acknowledgements}

The authors wish to thank Dr. Heinz Gögelein, Sanofi-Aventis Germany GmbH, Frankfurt, Germany for supplying HMR1556, and Dr. András Varró, University of Szeged, Szeged, Hungary for supplying SEA0400. Technical support by Roel L.H.M.G. Spätjens, BSc, Cardiovascular Research Institute Maastricht, The Netherlands and Ann J. Woods, Safety Pharmacology, SAUK, Astrazeneca R\&D, UK is greatly appreciated. 


\section{References}

(1) Thomsen MB, Verduyn SC, Stengl M, Beekman JD, de Pater G, van Opstal J et al. Increased short-term variability of repolarization predicts d-sotalol-induced torsades de pointes in dogs. Circulation. 2004;110:2453-59.

(2) Schneider J, Hauser R, Andreas JO, Linz K, Jahnel U. Differential effects of human ether-a-go-go-related gene (HERG) blocking agents on QT duration variability in conscious dogs. Eur J Pharmacol. 2005;512:53-60.

(3) Lengyel C, Varró A, Tábori K, Papp JG, Baczkó I. Combined pharmacological block of $\mathrm{I}_{\mathrm{Kr}}$ and $\mathrm{I}_{\mathrm{Ks}}$ increases short-term QT interval variability and provokes torsades de pointes. Br J Pharmacol. 2007;151:941-51.

(4) Wu L, Shryock JC, Song Y, Belardinelli L. An increase in late sodium current potentiates the proarrhythmic activities of low-risk QT-prolonging drugs in female rabbit hearts. J Pharmacol Exp Ther. 2006;316:718-26.

(5) Hinterseer M, Thomsen MB, Beckmann BM, Pfeufer A, Schimpf R, Wichmann $\mathrm{HE}$ et al. Beat-to-beat variability of QT intervals is increased in patients with drug-induced long-QT syndrome: a case control pilot study. Eur Heart J. 2008;29:185-90.

(6) Gallacher DJ, Van de Water A, van der Linde H, Hermans AN, Lu HR, Towart $\mathrm{R}$ et al. In vivo mechanisms precipitating torsades de pointes in a canine model of drug-induced long-QT1 syndrome. Cardiovasc Res. 2007;76:247-56.

(7) Thomsen MB, Volders PG, Beekman JD, Matz J, Vos MA. Beat-to-Beat variability of repolarization determines proarrhythmic outcome in dogs susceptible to drug-induced torsades de pointes. $J$ Am Coll Cardiol. 2006;48:1268-76.

(8) Zaniboni M, Pollard AE, Yang L, Spitzer KW. Beat-to-beat repolarization variability in ventricular myocytes and its suppression by electrical coupling. Am J Physiol. 2000;278:H677-87.

(9) Zaniboni M, Cacciani F, Salvarani N. Temporal variability of repolarization in rat ventricular myocytes paced with time-varying frequencies. Exp Physiol. 2007;92:859-69. 
(10) Volders PG, Stengl M, van Opstal JM, Gerlach U, Spätjens RL, Beekman JD et al. Probing the contribution of $\mathrm{I}_{\mathrm{Ks}}$ to canine ventricular repolarization: key role for beta-adrenergic receptor stimulation. Circulation. 2003;107:2753-60.

(11) Song Y, Shryock JC, Wu L, Belardinelli L. Antagonism by ranolazine of the pro-arrhythmic effects of increasing late INa in guinea pig ventricular myocytes. J Cardiovasc Pharmacol. 2004;44:192-99.

(12) Undrovinas AI, Belardinelli L, Undrovinas NA, Sabbah HN. Ranolazine improves abnormal repolarization and contraction in left ventricular myocytes of dogs with heart failure by inhibiting late sodium current. J Cardiovasc Electrophysiol. 2006;17:S169-S177.

(13) Varro A, Baláti B, Iost N, Takács J, Virág L, Lathrop DA et al. The role of the delayed rectifier component $\mathrm{I}_{\mathrm{Ks}}$ in dog ventricular muscle and Purkinje fibre repolarization. J Physiol. 2000;523:67-81.

(14) Jost N, Virág L, Bitay M, Takács J, Lengyel C, Biliczki P et al. Restricting excessive cardiac action potential and QT prolongation: a vital role for $\mathrm{I}_{\mathrm{Ks}}$ in human ventricular muscle. Circulation. 2005;112:1392-9.

(15) Carmeliet E. Repolarization reserve in cardiac cells. Journal of Med Biol Eng. 2006;26:97-105.

(16) Roden DM. Taking the "idio" out of "idiosyncratic": predicting torsades de pointes. Pacing Clin Electrophysiol. 1998;21:1029-34.

(17) Volders PG, Sipido KR, Carmeliet E, Spätjens RL, Wellens HJ, Vos MA. Repolarizing $\mathrm{K}^{+}$currents $\mathrm{I}_{\mathrm{TO} 1}$ and $\mathrm{I}_{\mathrm{Ks}}$ are larger in right than left canine ventricular midmyocardium. Circulation. 1999;99:206-10.

(18) Gögelein H, Brüggemann A, Gerlach U, Brendel J, Busch AE. Inhibition of $\mathrm{I}_{\mathrm{Ks}}$ channels by HMR 1556. Naunyn Schmiedebergs Arch Pharmacol. 2000;362:480-88.

(19) Thomas GP, Gerlach U, Antzelevitch C. HMR 1556, a potent and selective blocker of slowly activating delayed rectifier potassium current. J Cardiovasc Pharmacol. 2003;41:140-47.

(20) Birinyi $P$, Acsai $K$, Bányász $T$, Tóth $A$, Horváth $B$, Virág $L$ et al. Effects of SEA0400 and KB-R7943 on $\mathrm{Na}^{+} / \mathrm{Ca}^{2+}$ exchange current and L-type $\mathrm{Ca}^{2+}$ current in canine ventricular cardiomyocytes. Naunyn Schmiedebergs Arch Pharmacol. 2005;372:63-70. 
(21) Birinyi P, Tóth A, Jóna I, Acsai K, Almássy J, Nagy $\mathrm{N}$ et al. The $\mathrm{Na}^{+} / \mathrm{Ca}^{2+}$ exchange blocker SEA0400 fails to enhance cytosolic $\mathrm{Ca}^{2+}$ transient and contractility in canine ventricular cardiomyocytes. Cardiovasc Res. 2008;78:476-84.

(22) Volders PG, Sipido KR, Vos MA, Kulcsár A, Verduyn SC, Wellens HJ. Cellular basis of biventricular hypertrophy and arrhythmogenesis in dogs with chronic complete atrioventricular block and acquired torsade de pointes. Circulation. 1998;98:1136-47.

(23) Stengl M, Volders PG, Thomsen MB, Spätjens RL, Sipido KR, Vos MA. Accumulation of slowly activating delayed rectifier potassium current $\left(\mathrm{I}_{\mathrm{Ks}}\right)$ in canine ventricular myocytes. J Physiol. 2003;551:777-86.

(24) Volders PG, Kulcśar A, Vos MA, Sipido KR, Wellens HJ, Lazzara R et al. Similarities between early and delayed afterdepolarizations induced by isoproterenol in canine ventricular myocytes. Cardiovasc Res. 1997;34:348-59.

(25) Volders PG, Vos MA, Szabo B, Sipido KR, de Groot SH, Gorgels AP et al. Progress in the understanding of cardiac early afterdepolarizations and torsades de pointes: time to revise current concepts. Cardiovasc Res. 2000;46:376-92.

(26) Linz KW, Meyer R. Control of L-type calcium current during the action potential of guinea-pig ventricular myocytes. J Physiol. 1998;513:425-42.

(27) Tang Q, Jin MW, Xiang JZ, Dong MQ, Sun HY, Lau CP et al. The membrane permeable calcium chelator BAPTA-AM directly blocks human ether a-go-gorelated gene potassium channels stably expressed in HEK 293 cells. Biochem Pharmacol . 2007;74:1596-607.

(28) Wan X, Laurita KR, Pruvot EJ, Rosenbaum DS. Molecular correlates of repolarization alternans in cardiac myocytes. $\mathrm{J} \mathrm{Mol}$ Cell Cardiol. 2005;39:419-28.

(29) Kass RS. Genetically induced reduction in small currents has major impact. Circulation. 1997;96:1720-1721.

(30) Tanskanen AJ, Greenstein JL, O'Rourke B, Winslow RL. The role of stochastic and modal gating of cardiac L-type $\mathrm{Ca}^{2+}$ channels on early after-depolarizations. Biophys J. 2005;88:85-95. 
(31) Burashnikov A, Antzelevitch C. Block of $\mathrm{I}_{\mathrm{Ks}}$ does not induce early afterdepolarization activity but promotes beta-adrenergic agonist-induced delayed afterdepolarization activity. J Cardiovasc Electrophysiol. 2000;11:458-65.

(32) Milberg P, Pott C, Fink M, Frommeyer G, Matsuda T, Baba A et al. Inhibition of the $\mathrm{Na}^{+} / \mathrm{Ca}^{2+}$ exchanger suppresses torsades de pointes in an intact heart model of long QT syndrome-2 and long QT syndrome-3. Heart Rhythm. 2008;5:1444-52.

(33) Farkas AS, Makra P, Csík N, Orosz S, Shattock MJ, Fülöp F et al. The role of the $\mathrm{Na}^{+} / \mathrm{Ca}^{2+}$ exchanger, $\mathrm{I}_{\mathrm{Na}}$ and $\mathrm{I}_{\mathrm{CaL}}$ in the genesis of dofetilide-induced torsades de pointes in isolated, AV-blocked rabbit hearts. $\mathrm{Br} \mathrm{J}$ Pharmacol. 2009 Feb 16. [Epub ahead of print].

(34) Shimizu W, Kurita T, Matsuo K, Suyama K, Aihara N, Kamakura S et al. Improvement of repolarization abnormalities by a $\mathrm{K}^{+}$channel opener in the LQT1 form of congenital long-QT syndrome. Circulation. 1998 ;97:1581-8.

(35) Satomi K, Shimizu W, Takaki H, Suyama K, Kurita T, Aihara N et al. Response of beat-by-beat QT variability to sympathetic stimulation in the LQT1 form of congenital long QT syndrome. Heart Rhythm. 2005;2:149-54.

(36) Shimizu W, Ohe T, Kurita T, Kawade M, Arakaki Y, Aihara N et al. Effects of verapamil and propranolol on early afterdepolarizations and ventricular arrhythmias induced by epinephrine in congenital long QT syndrome. J Am Coll Cardiol. 1995;26:1299-309.

(37) Khositseth A, Nemec J, Hejlik J, Shen WK, Ackerman MJ. Effect of phenylephrine provocation on dispersion of repolarization in congenital long QT syndrome. Ann Noninvasive Electrocardiol. 2003;8:208-14. 
Chapter 4

\section{Diastolic Spontaneous Calcium Release from the Sarcoplasmic Reticulum I ncreases Beat-to-Beat Repolarization Variability in Canine Ventricular Myocytes after $\boldsymbol{\beta}$-Adrenergic Stimulation}

Circulation Research. 2013;112(2):246-56

$\mathrm{DMJ}$ and JH contributed equally to this work

Daniel M Johnson • Jordi Heijman • Elizabeth F Bode • David J Greensmith • Henk van der Linde • Najah Abi-Gerges • David A Eisner Andrew W Trafford $•$ Paul GA Volders 


\section{Abstract}

Spontaneous $\mathrm{Ca}^{2+}$ release from the sarcoplasmic reticulum (SCR) can cause delayed afterdepolarizations (DADs) and triggered activity, contributing to arrhythmogenesis during $\beta$-adrenergic receptor ( $\beta A R$ ) stimulation. Excessive beat-to-beat variability of repolarization duration (BVR) is a proarrhythmic marker. Previous research has shown that BVR is increased during intense $\beta A R$ stimulation leading to SCR.

We aimed to determine ionic mechanisms controlling BVR under these conditions. Membrane potentials and cell shortening or $\mathrm{Ca}^{2+}$ transients were recorded from isolated canine left-ventricular myocytes in the presence of isoproterenol. Actionpotential (AP) durations following DADs were significantly prolonged. Addition of $\mathrm{I}_{\mathrm{ks}}$ blockade led to further AP prolongation after SCR and this strongly correlated with exaggerated BVR. Suppressing SCR via inhibition of ryanodine receptors, CaMKII inhibition, or by using $\mathrm{Mg}^{2+}$ or flecainide, eliminated DADs and decreased BVR independent of effects on AP duration. Computational analyses and voltage-clamp experiments measuring $\mathrm{I}_{\mathrm{CaL}}$ with and without prior $\mathrm{SCR}$ indicated that $\mathrm{I}_{\mathrm{CaL}}$ was increased during $\mathrm{Ca}^{2+}$-induced $\mathrm{Ca}^{2+}$ release after $\mathrm{SCR}$, and this contributes to AP prolongation. Prolongation of QT, $\mathrm{T}_{\text {peak }}-\mathrm{T}_{\text {end }}$ intervals and left-ventricular monophasic AP duration of beats following aftercontractions occurred prior to torsades de pointes in an in-vivo dog model of drug-induced long-QT1 syndrome.

SCR contributes to increased BVR by interspersed prolongation of AP duration, which is exacerbated during $\mathrm{I}_{\mathrm{Ks}}$ blockade. Attenuation of $\mathrm{Ca}^{2+}$-induced $\mathrm{Ca}^{2+}$ release by $\mathrm{SCR}$ underlies AP prolongation via increased $\mathrm{I}_{\mathrm{CaL}}$. These data provide novel insights into arrhythmogenic mechanisms during $\beta A R$ stimulation besides triggered activity and illustrate the importance of $\mathrm{I}_{\mathrm{Ks}}$ function in preventing excessive BVR. 


\subsection{I ntroduction}

Enhanced cellular $\mathrm{Ca}^{2+}$ load, for example during $\beta$-adrenergic receptor ( $\beta A R$ ) stimulation, results in augmentation of $\mathrm{Ca}^{2+}$ release from the sarcoplasmic reticulum (SR), larger $\mathrm{Ca}^{2+}$ transients (CaT), and enhanced contractile force ${ }^{1}$. Under certain conditions $\mathrm{Ca}^{2+}$ load is increased beyond a certain threshold, leading to spontaneous $\mathrm{Ca}^{2+}$ release (SCR) from the SR during diastole ${ }^{2}$. In turn, this diastolic leak causes a transient inward current $\left(\mathrm{I}_{\mathrm{TI}}\right)$, causing delayed afterdepolarizations (DADs), mainly due to activation of the electrogenic $\mathrm{Na}^{+}-\mathrm{Ca}^{2+}$ exchanger (NCX), with the $\mathrm{Ca}^{2+}$-activated $\mathrm{Cl}^{-}$current $\left(\mathrm{I}_{\mathrm{CI}(\mathrm{Ca})}\right)$ contributing in some species ${ }^{3,4}$. Previous work has shown that both DADs and early afterdepolarizations (EADs) may share a common mechanism, at least during $\beta A R$ stimulation and $\mathrm{Ca}^{2+}$ overload, namely SCR-induced currents ${ }^{5}$. Both types of afterdepolarizations have been incriminated in the formation of ventricular tachycardia (VT), via triggered activity (TA) and/or by increasing dispersion of repolarization ${ }^{6}$.

Beat-to-beat variability of repolarization duration (BVR) occurs as an apparently random alteration in repolarization duration, and can be observed at all levels, from the action potential (AP) of the single cardiac myocyte to the QT interval on the body surface $^{7-9}$. Exaggerated BVR has been reported to be a more reliable indicator of arrhythmogenic risk than repolarization prolongation per se, at least in several experimental VT models ${ }^{10-12}$ and in selected human subjects ${ }^{8,13}$.

Although BVR has been investigated in multiple studies, the mechanisms underlying this phenomenon at the single-cell level remain to be fully elucidated. Pharmacological interventions influencing ion channels that operate during the AP plateau can markedly alter BVR ${ }^{7,14}$. Despite the fact that inhibition of the slowlyactivating delayed-rectifier $\mathrm{K}^{+}$current $\left(\mathrm{I}_{\mathrm{Ks}}\right)$ alone has minimal effects on both cellular AP duration (APD) and BVR ${ }^{14}$, we have recently shown that during increased $\mathrm{Ca}^{2+}$ loading in myocytes subjected to blockade of $\mathrm{I}_{\mathrm{ks}}$ in combination with $\beta A R$ stimulation, $B V R$ is significantly enhanced, even before the occurrence of EADs and TA ${ }^{14}$.

In the present study, we investigated the relationship between SCR and BVR using a combined experimental and computational approach in both canine ventricular myocytes and in-situ hearts subjected to BAR stimulation. We show that SCRs not only lead to $I_{T I}$ and DAD formation, but also to a prolonged duration of the following $A P$ via increased L-type $\mathrm{Ca}^{2+}$ current $\left(\mathrm{I}_{\mathrm{CaL}}\right)$, which in turn leads to increased $\mathrm{BVR}$ when analyzing multiple consecutive APs. Pharmacological interventions that inhibit SCR (either with reduced or preserved systolic contraction) prevent this SCRassociated AP prolongation and reduce BVR. 


\subsection{Methods}

This investigation conformed to the Guide for the Care and Use of Laboratory Animals published by the US National Institutes of Health (NIH Publication No. 85-23, revised 1996). Animal handling was in accordance with the European Directive for the Protection of Vertebrate Animals Used for Experimental and Other Scientific Purposes (86/609/EU).

\subsubsection{Myocyte I solation and Electrophysiology}

Canine left ventricular myocytes were isolated as previously described ${ }^{15}$. Transmembrane APs were recorded at $\sim 37^{\circ} \mathrm{C}$ using high-resistance (30-60 M $\Omega$ ) glass microelectrodes filled with $3 \mathrm{~mol} / \mathrm{L} \mathrm{KCl}$. Myocyte contractions were recorded with a video edge motion detector.

\subsubsection{Calcium Measurement}

We used the perforated patch-clamp technique under current- or voltage-clamp control as previously described ${ }^{16}$. Myocytes were stimulated to elicit APs (current clamp) or $\mathrm{I}_{\mathrm{CaL}}$ (voltage clamp). Changes in intracellular $\mathrm{Ca}^{2+}$ concentration $\left(\left[\mathrm{Ca}^{2+}\right]_{\mathrm{i}}\right)$ were measured using Fluo-3 or Fura-2 AM ${ }^{16}$.

\subsubsection{Computational Analysis}

A recent model of the canine ventricular myocyte electrophysiology including BAR stimulation ${ }^{17}$ was extended with a method to induce diastolic $\mathrm{SR} \mathrm{Ca}^{2+}$ release in one of two identical $\mathrm{Ca}^{2+}$ domains in a controlled fashion (Figures $\mathbf{1}$ and 2). Simulations were performed in single cells as well as a one-dimensional homogeneous strand or two-dimensional tissue of electrically-coupled cells to determine the effects of diastolic $\mathrm{Ca}^{2+}$ release in the presence of electrotonic coupling. 


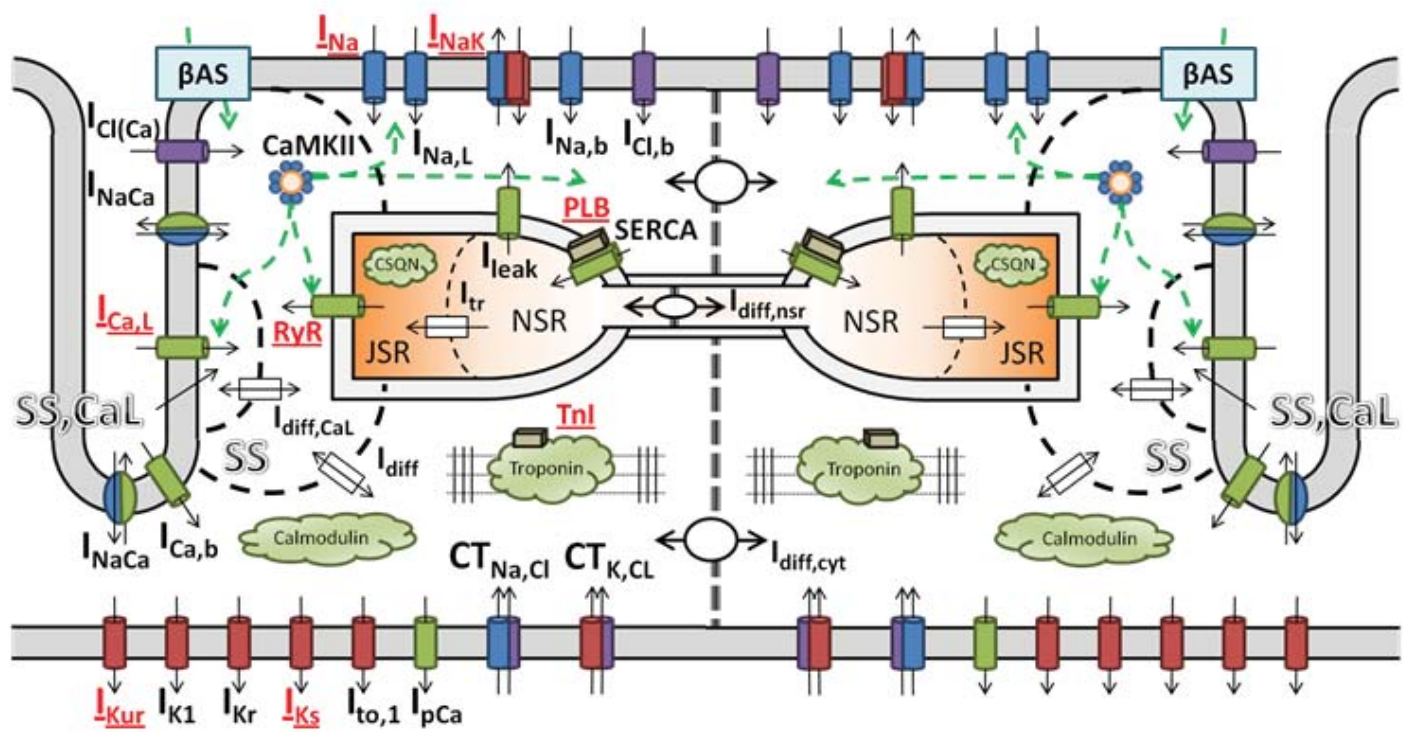

Figure 1: Schematic overview of the computational model of the canine ventricular myocyte. Adapted from Heijman et al. ${ }^{17}$. The model was divided into two identical domains to simulate the local origin of SCR. Only components on the left side of the model are labeled for clarity, identical components are located on the right side. The domains are coupled via diffusion of $\mathrm{Ca}^{2+}\left(\mathrm{I}_{\text {diff,cyt }}\right.$ and $\left.\mathrm{I}_{\text {diff,nsr }}\right)$. All other abbreviations are as previously described ${ }^{17}$.

Similar simulations were performed in a one-dimensional homogeneous strand of electrically-coupled cells to determine the effects of diastolic $\mathrm{Ca}^{2+}$ release in the presence of electrotonic coupling.

\subsubsection{In-Vivo Dog Model of Drug-I nduced Long-QT1 Syndrome}

Torsades de Pointes (TdP) arrhythmias were induced in an in-vivo dog model of long-QT1 (LQT1) syndrome, as previously described. ${ }^{11}$ ECG, left ventricular pressure (LVP) and left ventricular monophasic action potentials (LV MAPs) were recorded throughout the experiments and analyzed offline. 
$A \mathrm{APD}_{90}$ rate dependence (no DAD)
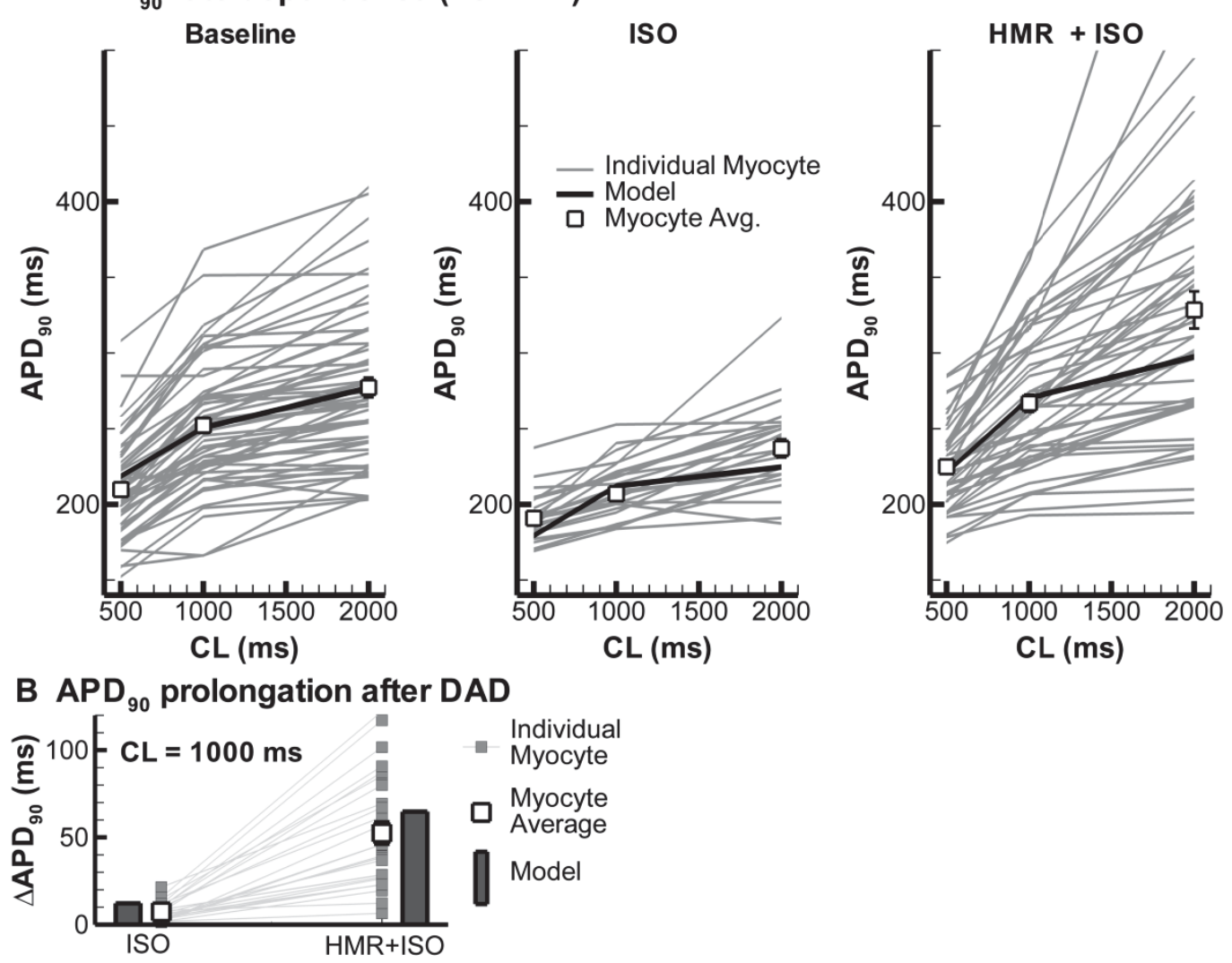

Figure 2: Validation of model properties. A. Steady-state APD-rate dependence under baseline conditions (left panel), in the presence of ISO (middle panel) or in the presence of ISO + HMR1556 (right panel) for APs without prior DAD. Average APD for each cell is shown in grey. Average APD over all myocytes is indicated with symbols. Model APD is shown in black. Model APD-rate dependence falls within experimental range and is close to the experimental average for all conditions and CLs. B. Average APD difference between APS preceded by a DAD and those without prior DAD in the presence of ISO or ISO + HMR1556. Average APD difference for each cell is shown in grey. Average group data is indicated with symbols. Model APD prolongation after SCR is indicated with grey bars. Model APD prolongation is consistent with experimental observations.

\subsubsection{Data Analysis and Statistical Comparisons}

APD was quantified at $90 \%$ repolarization. BVR was quantified as variability of APD using the formula $\Sigma(|A P D i+1-A P D i|) /[$ nbeats $\times \sqrt{ } 2]$ for 30 consecutive APs in the absence of TA ${ }^{10}$. DADs were detected with a semi-automated method using a custom software script. Where applicable, data were reported as mean \pm SEM of $n$ experiments, and significance tested with either a t-test or one-way ANOVA. Differences were considered statistically significant if $\mathrm{P}<0.05$. 


\subsection{Results}

\subsubsection{DADs Prolong Subsequent APs and I ncrease BVR}

Under baseline conditions, a small beat-to-beat APD variability was observed (Figure 3A; left panel). BAR stimulation with isoproterenol (ISO) shortened APD and enhanced contraction (Figure 3A; middle panel). In the majority of cells, 100 $\mathrm{nmol} / \mathrm{L}$ ISO caused DADs during pacing at $1000 \mathrm{~ms} C L$. APs following a DAD were prolonged, leading to increased BVR due to interspersed occurrence of normal and prolonged APs (Figure 3A; middle panel). Pharmacological inhibition of $\mathrm{I}_{\mathrm{Ks}}$ by HMR1556 (500 nmol/L) in the presence of ISO further prolonged APD and increased BVR (Figure 3A; right panel). In separate experiments, $\mathrm{Ca}^{2+}$ transients were recorded together with APs after HMR1556 and ISO. HMR1556 alone did not increase the $\mathrm{Ca}^{2+}$-transient amplitude nor lead to any SCR events. The combination of HMR1556 and ISO, however, led to large increases in $\mathrm{Ca}^{2+}$ amplitude and SCR, as expected. ${ }^{18}$ APs following SCR were prolonged and had a reduced systolic $\mathrm{Ca}^{2+}$ transient amplitude compared to those where no diastolic release preceded the AP, indicating reduced SR $\mathrm{Ca}^{2+}$ load at the start of the AP (Figure 3B), consistent with the reduced cell shortening following an aftercontraction (Figure $\mathbf{3 A}$ ).

APD prolongation following a DAD was significantly more pronounced in the presence of ISO + HMR1556 compared to ISO alone at all CLs (e.g., $41 \pm 6 \mathrm{~ms}$ vs. $15 \pm 4 \mathrm{~ms}$ at $\mathrm{CL}=1000 \mathrm{~ms}, \mathrm{P}<0.05$, Figure 4A) and this coincided with a significant increase in BVR (17 \pm 2 ms vs. $7 \pm 1 \mathrm{~ms} ; \mathrm{P}<0.05$, Figure 4B). As such, HMR1556 was added in all subsequent experiments in the presence of ISO to maximize effects on APD, essentially creating a cellular model of drug-induced LQT1 syndrome ${ }^{14}$. DADs showed typical rate-dependent properties, including increased amplitude and decreased AP-DAD coupling interval at shorter pacing $\mathrm{CL}$, consistent with previous results ${ }^{19}$. $\mathrm{I}_{\mathrm{Ks}}$ blockade did not alter rate-dependent DAD properties (Figure 5). 

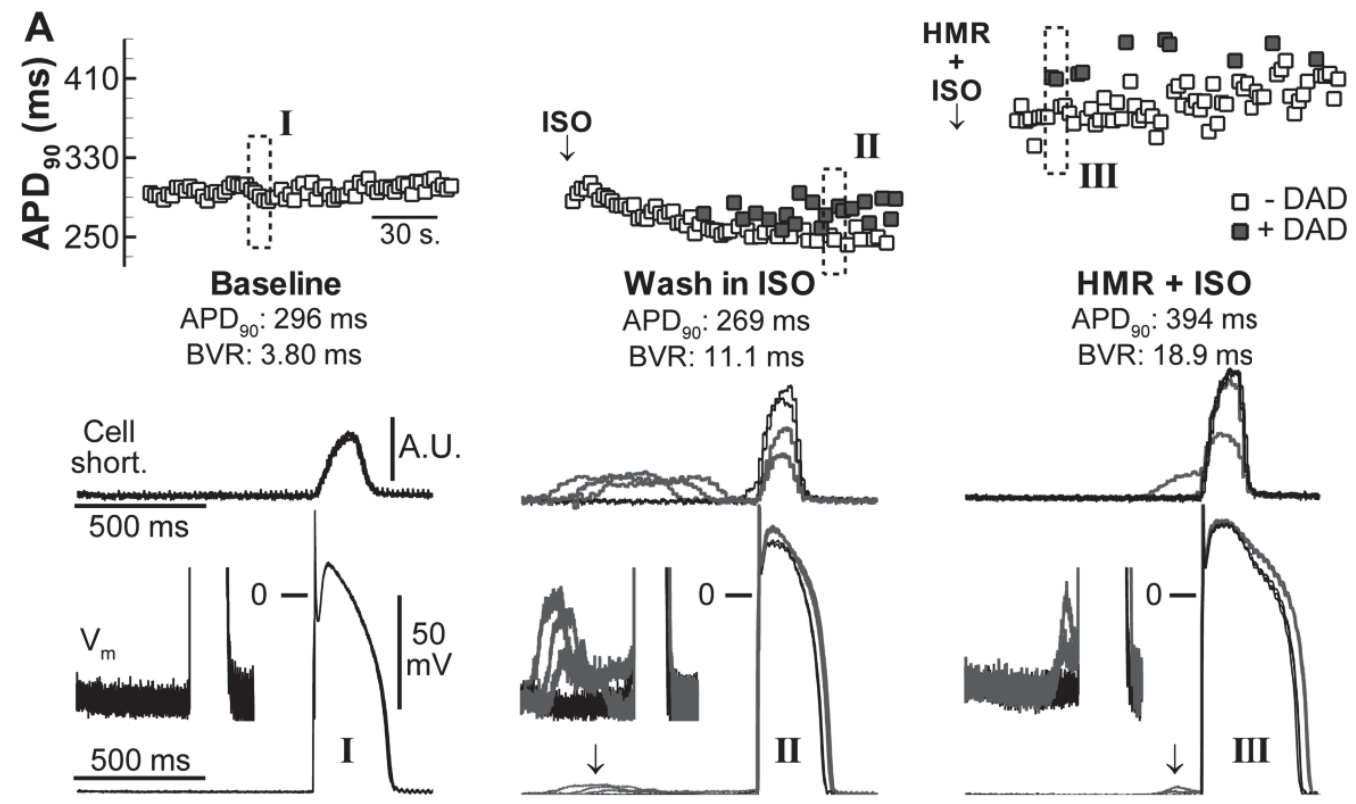

B

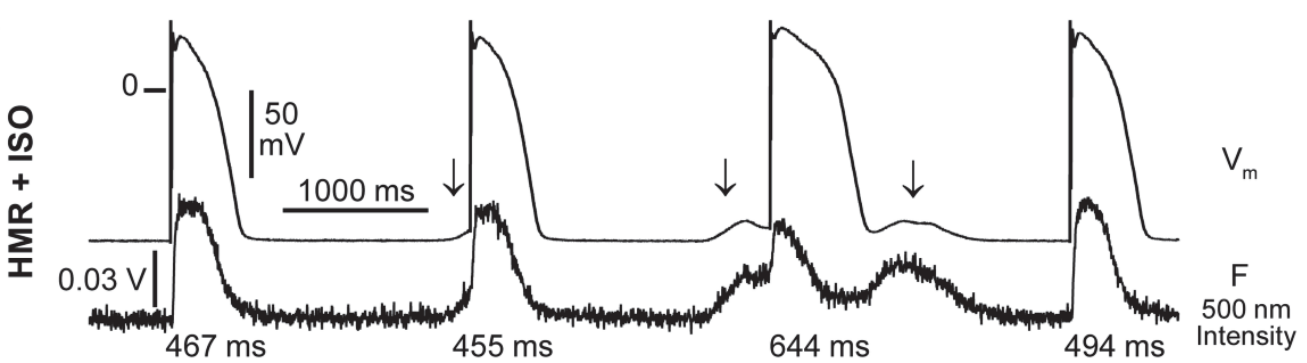

Figure 3: Occurrence of DADs in canine ventricular myocytes is associated with prolongation of subsequent APs and increased BVR. A. APD 90 for 75 consecutive beats at 2000-ms CL at baseline (left panel: I), during wash-in of ISO (middle panel: II) and in the presence of HMR1556 and ISO (right panel: III). APDs preceded by a DAD are indicated with filled symbols, APDs without DADs depicted by open symbols. Dashed rectangle indicates a set of 5 beats for which cell shortening and membrane potential are depicted below. Insets show diastolic potentials at an expanded scale. Beats without prior SCR are indicated in black, beats with prior SCR in grey. B. Membrane potential (top) and $\left[\mathrm{Ca}^{2+}\right]_{i}$ for four consecutive beats. $\mathrm{APD}_{90} \mathrm{~S}$ are indicated below each AP. SCR is indicated with arrows.

To determine whether DAD-like membrane-potential changes alone (i.e., without underlying alterations in intracellular $\mathrm{Ca}^{2+}$ ) influenced the subsequent $\mathrm{AP}$, current was injected to induce small alterations in the resting membrane potential (25-mV amplitude, 150-ms duration; Figure 4C; top panel). There was no significant difference between APDs in the absence or presence of these diastolic pulses (Figure 4D), independent of the timing of the pulse (not shown). In contrast, when these cells were stimulated with HMR1556 + ISO, DADs occurred and subsequent APD was significantly prolonged (Figure 4C; bottom panel), indicating that alterations in the subsequent APD were influenced by DAD-related events other than membrane-potential alterations per se. 


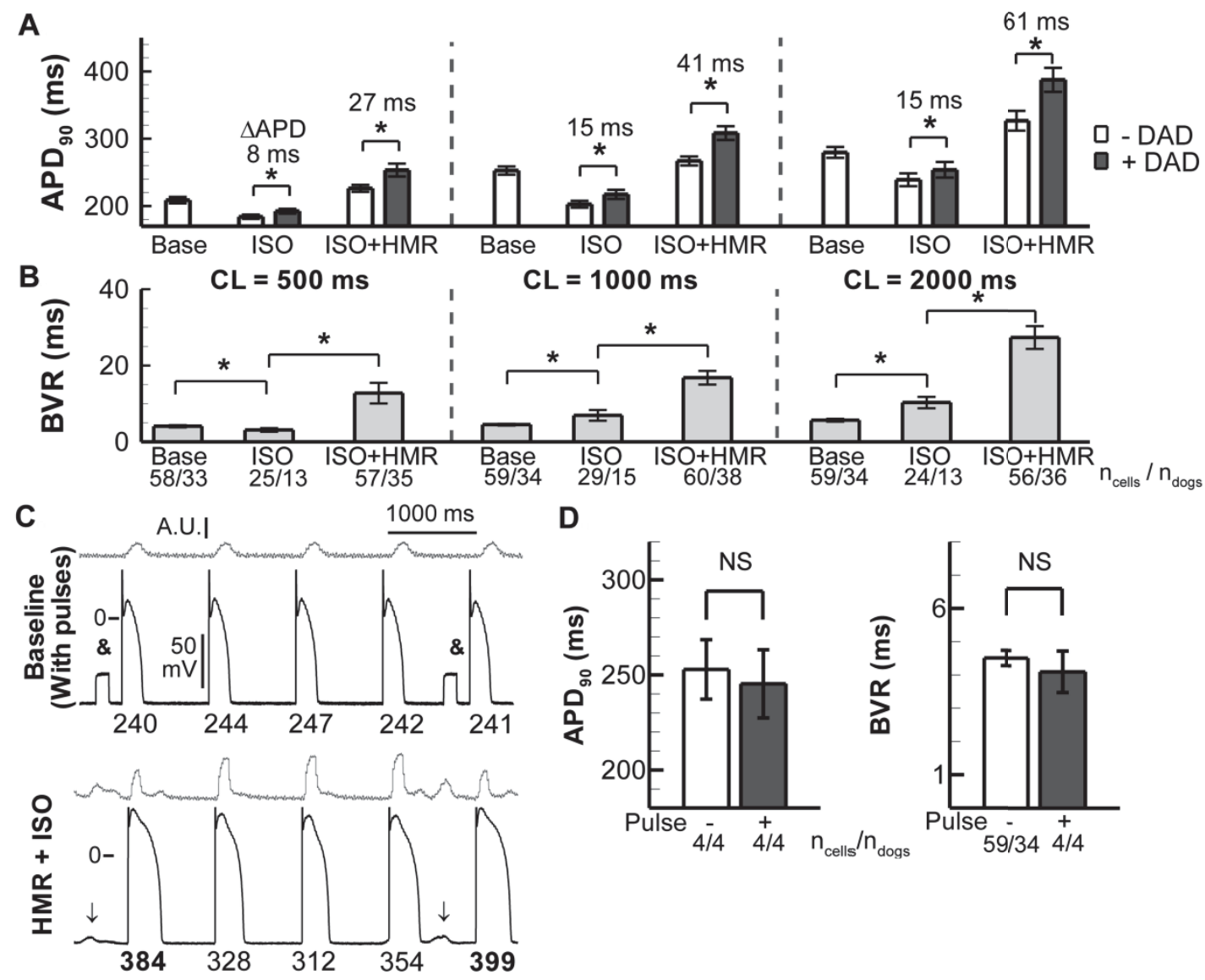

Figure 4: A. Group data for $\mathrm{APD}_{90}$ under baseline conditions, in the presence of ISO, or in the presence of ISO + HMR1556 at CLs of 500, 1000 and 2000 ms. DADs cause a significant prolongation during ISO and ISO + HMR1556 treatment. APD ${ }_{90}$ differences are indicated above each pair of bars. *; $\mathrm{P}<0.05$ based on paired T-test. B. BVR values for the conditions in panel $A$. The number of cells and number of dogs for each condition are indicated below each bar. Comparisons between conditions were made using ANOVA. C. Cell shortening (top trace) and membrane potential (bottom trace) under baseline conditions with DAD-like electrical pulses (in the absence of SCR) applied every $4^{\text {th }}$ beat (top panel) or with DADs after ISO + HMR1556 in the same cell (bottom panel). APD 90 is indicated below each beat. D. Subthreshold diastolic pulses do not alter $A \mathrm{PD}_{90}$ within the same cell (left panel) nor BVR compared to cells without diastolic pulses (right panel). 
A

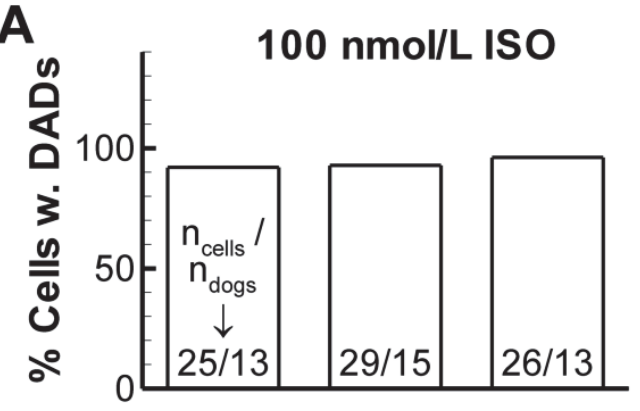

B

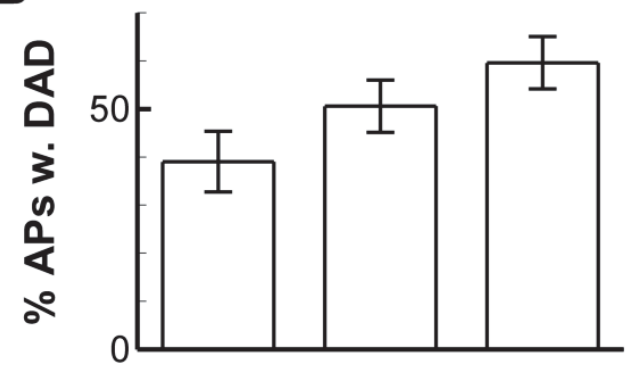

C

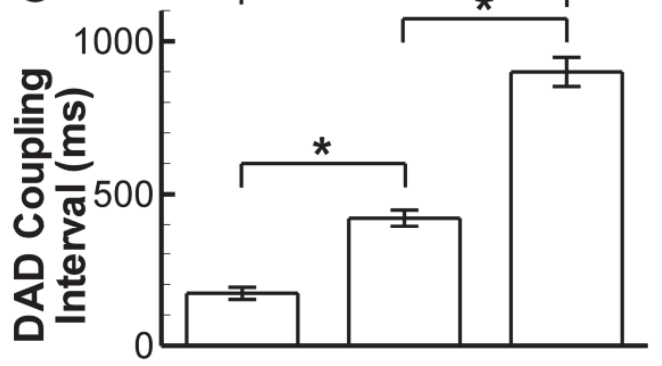

D

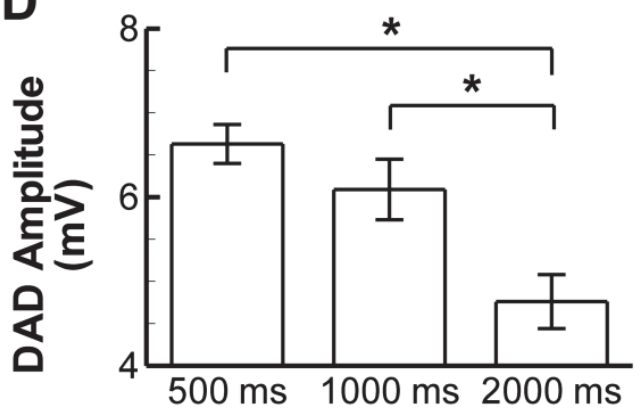

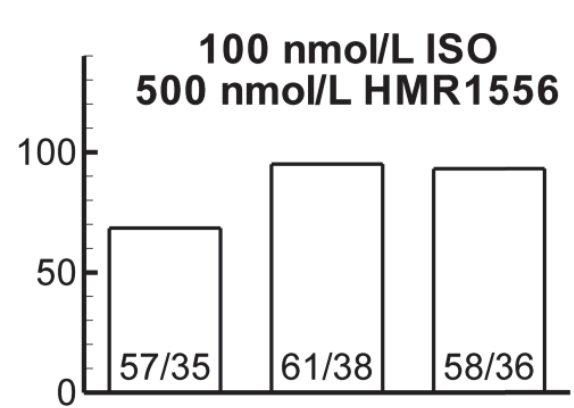

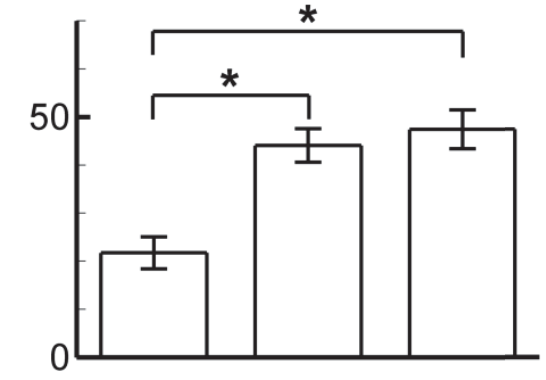

*

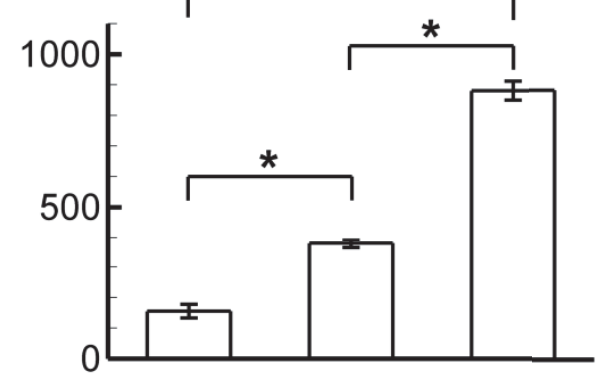

CL

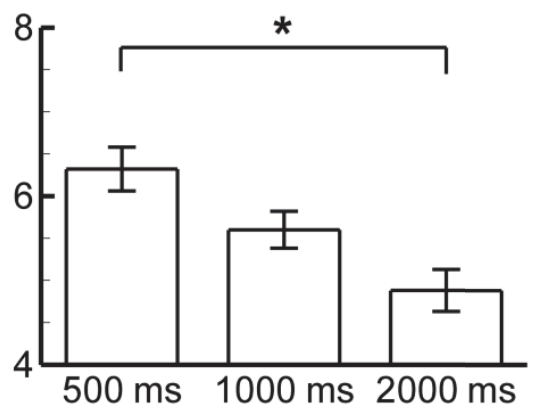

Figure 5: Rate-dependent characteristics of DADs in the presence of ISO (left panels) or ISO + HMR1556 (right panels). A. Percentage of cells which showed DADs during BAR stimulation. Number of cells and number of dogs are indicated in each bar. B. Average percentage of APs which had a DAD. C. Coupling interval between end of repolarization $\left(A P D_{90}\right)$ and subsequent $D A D$ for those beats which showed a DAD. D. Average DAD amplitude. * indicates $\mathrm{P}<0.05$ based on one-way ANOVA with Tukey-Kramer post-hoc test. DADs occur earlier and are larger at faster rates. Data are consistent with those of Priori and Corr ${ }^{20}$ in canine ventricular myocytes measured using a pace-pause protocol. 


\subsubsection{Coupling Between the Occurrence of DADs and EADs During $\beta$-Adrenergic Stimulation and $I_{\mathrm{Ks}}$ Blockade}

In addition to DADs, EADs were observed. EADs occurred predominantly at 500-ms $\mathrm{CL}$ and exclusively in the presence of HMR1556 + ISO.

A

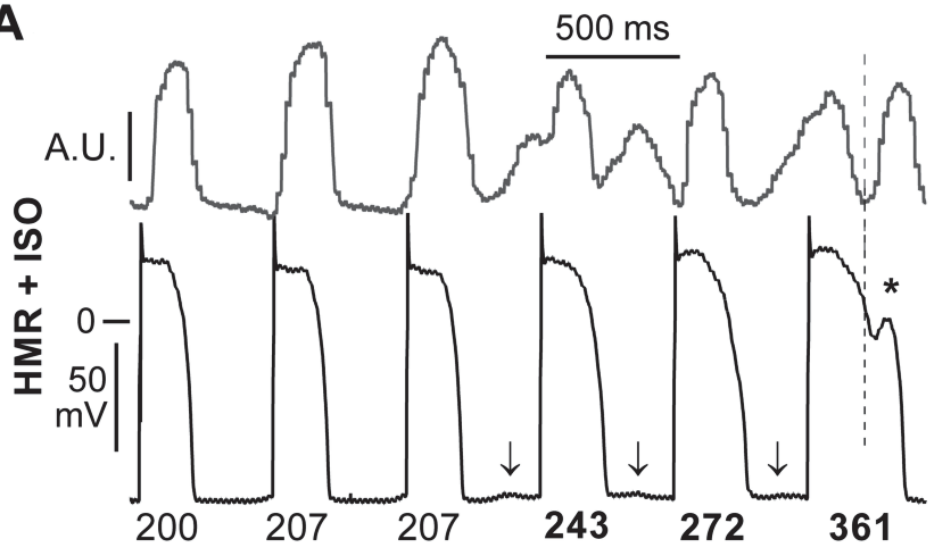

AP \# (relative to EAD)

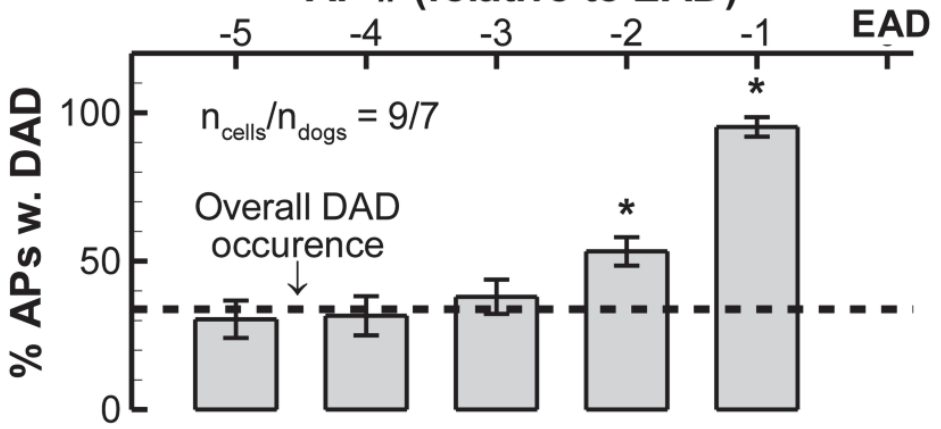

B

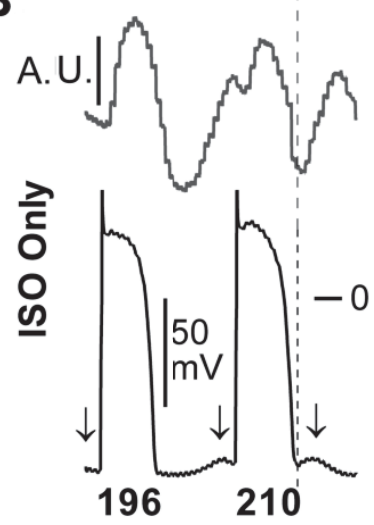

C

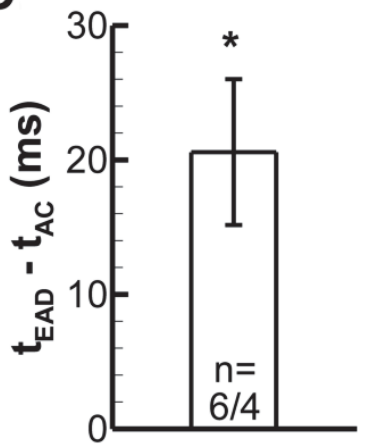

Figure 6: Relationship between diastolic SCR and EADs. A.Top panel: Cell shortening (top trace) and membrane potential (bottom trace) for 5 consecutive APs followed by an AP with EAD (indicated with $*$ ) in a representative cell at $500-\mathrm{ms} \mathrm{CL}$ in the presence of HMR1556 + ISO. $A P D_{90}$ is given below each beat and DADs are indicated with arrows. Vertical line indicates timing of start of aftercontraction. Bottom panel indicates the percentages of APs that showed a DAD in the 5 beats preceding every EAD. Horizontal dashed line indicates overall DAD occurrence at $500-\mathrm{ms} \mathrm{CL}$ in the presence of HMR1556 + ISO, independent of EADs. The beat before an EAD has a DAD 95\% of the time. *: $P<0.05$ compared to overall DAD occurrence. B. Similar to panel A (top) for 2 beats in the presence of ISO only. A similar contraction pattern can be observed but APD prolongation is insufficient to promote EAD formation. C. Time differences between rise of aftercontraction and $E A D$, indicating that aftercontractions occur earlier than EADs.

We observed early aftercontractions preceding EAD upstrokes by $21 \pm 5$ ms (Figure 6 A, $\mathbf{C}$ and ${ }^{5}$ ), consistent with the observations by Zhao et al. in rabbit ventricular myocytes challenged with ISO and the $\mathrm{I}_{\mathrm{CaL}}$ agonist Bay $\mathrm{K} 8644,{ }^{20}$ suggesting a role of SCR for EAD formation under these specific conditions ${ }^{5}$. 
We quantified the occurrence of DADs in the beats preceding an EAD and found that DADs were significantly more likely to occur in the 2 beats directly preceding an EAD ( $53 \%$ and $95 \%$ of APs) compared to average DAD occurrence for all beats (34\%; Figure 6A bottom panel). Figure 6B shows the responses during ISO alone.

\subsubsection{Blockade of (Spontaneous) SR $\mathrm{Ca}^{2+}$ Release Reduces BVR}

We sought to further investigate how eliminating SCR would affect APD and BVR. Blockade of the ryanodine receptor (RyR) with ryanodine eliminated DADs and led to a drastic reduction in BVR, despite leading to an increased APD (Figure 7A, B). APD prolongation under these conditions was not associated with any arrhythmogenic events. A drastic reduction in systolic contraction was also seen, as expected, indicating that blockade of RyRs was achieved effectively.

Tetracaine reduces the open probability $\left(P_{0}\right)$ of RyR leading to a decrease in the frequency of SCR in unstimulated rat ventricular myocytes ${ }^{21}$ and the abolishment of ISO-induced SCR in voltage-clamped rat ventricular myocytes ${ }^{22}$. Similar to ryanodine, tetracaine led to an elimination of DADs in our experiments, and a reduction in BVR at all CLs. However, unlike ryanodine, cell shortening remained largely unaltered, indicating that systolic $\mathrm{Ca}^{2+}$ release was largely intact (Figure 7C, D). 
A

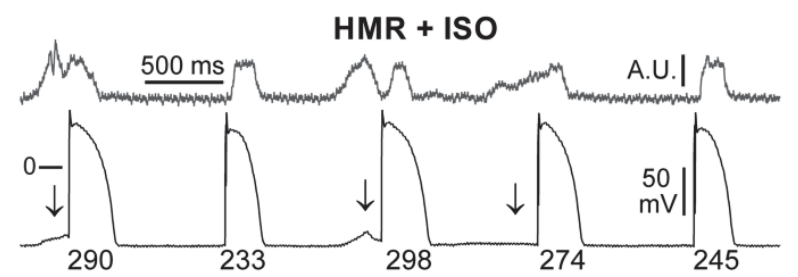

B

HMR + ISO + Ryanodine
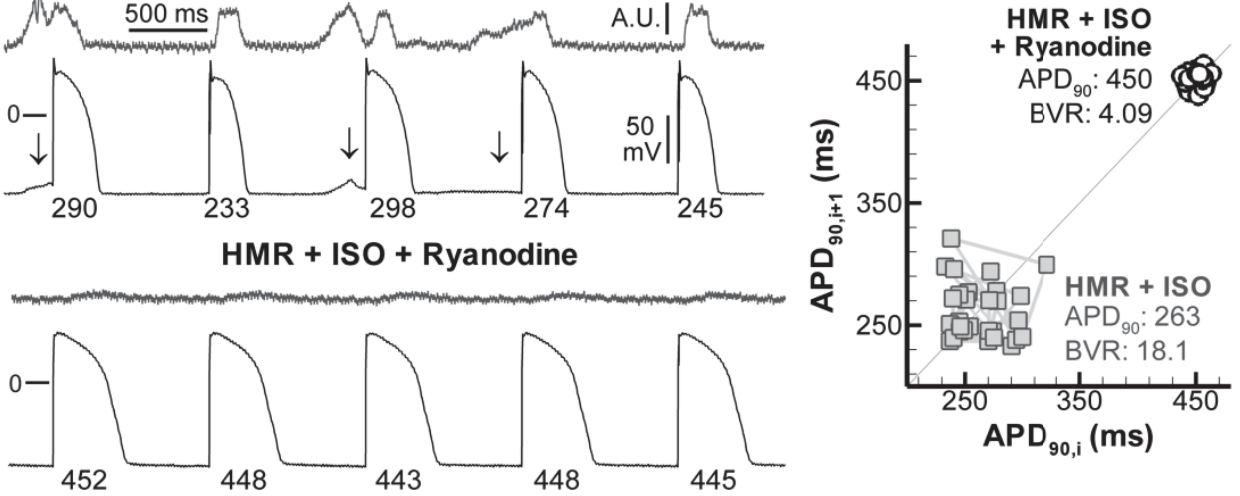

C

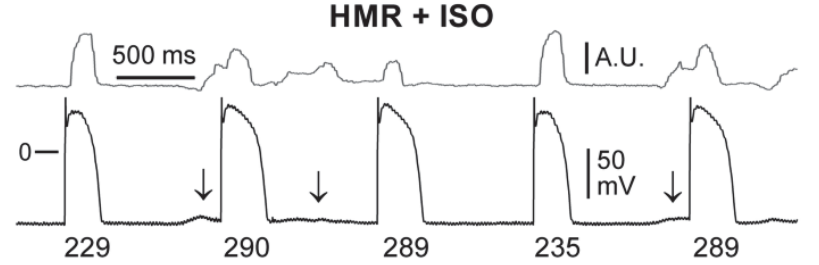

D

HMR + ISO + Tetracaine
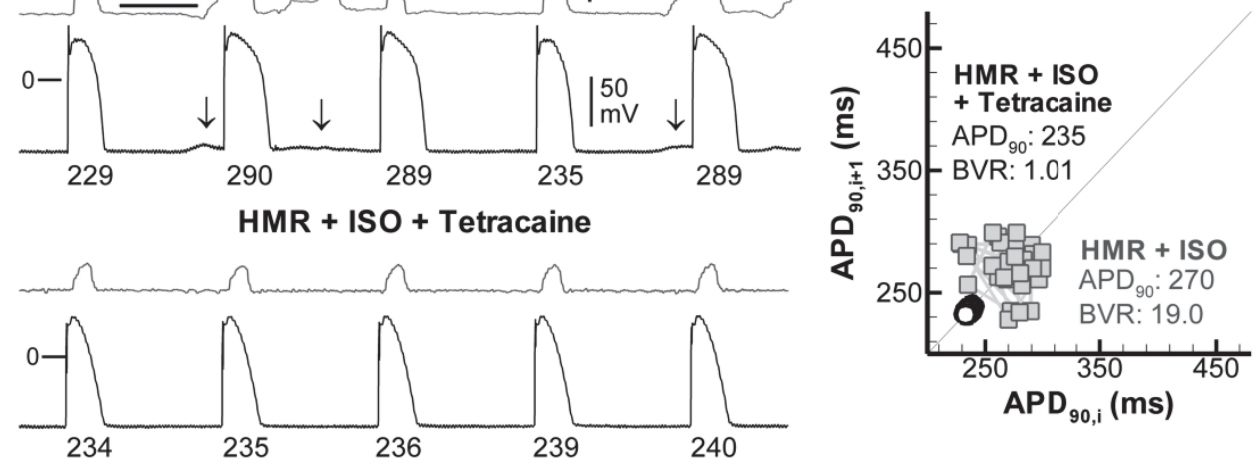

Figure 7: Inhibition of (diastolic) SCR abolishes DADs and reduces BVR. Cell shortening (top panels; arbitrary units) and membrane potential (bottom panels) for 5 representative beats during treatment with ISO + HMR1556 (A.), or ISO + HMR1556 + ryanodine (1 $\mu \mathrm{mol} / \mathrm{L})(\mathbf{B}$.$) .$ $A P D_{90}$ is indicated below each beat and mean $A P D_{90}$ and $B V R$ are shown for 30 beats in the Poincaré plot on the right. DADs are indicated with arrows. Panels $\mathbf{C}$. and $\mathbf{D}$. show similar data for treatment with tetracaine $(5 \mu \mathrm{mol} / \mathrm{L})$. ISO + HMR1556 significantly enhances BVR and DAD occurrence. Ryanodine and tetracaine abolish DADs and reduce BVR.

To determine the effects of increasing the $\mathrm{P}_{\mathrm{o}}$ of RyRs, as opposed to decreasing it, we used low concentrations of caffeine (maximally $500 \mu \mathrm{mol} / \mathrm{L}$ ) ${ }^{23}$. Under these conditions the percentage of APs showing DADs was increased at all CLs. Interestingly, this led to a significant decrease in BVR compared to HMR1556 and ISO alone, since DADs occurred before every AP (Figure 8, right-most grey bars). 


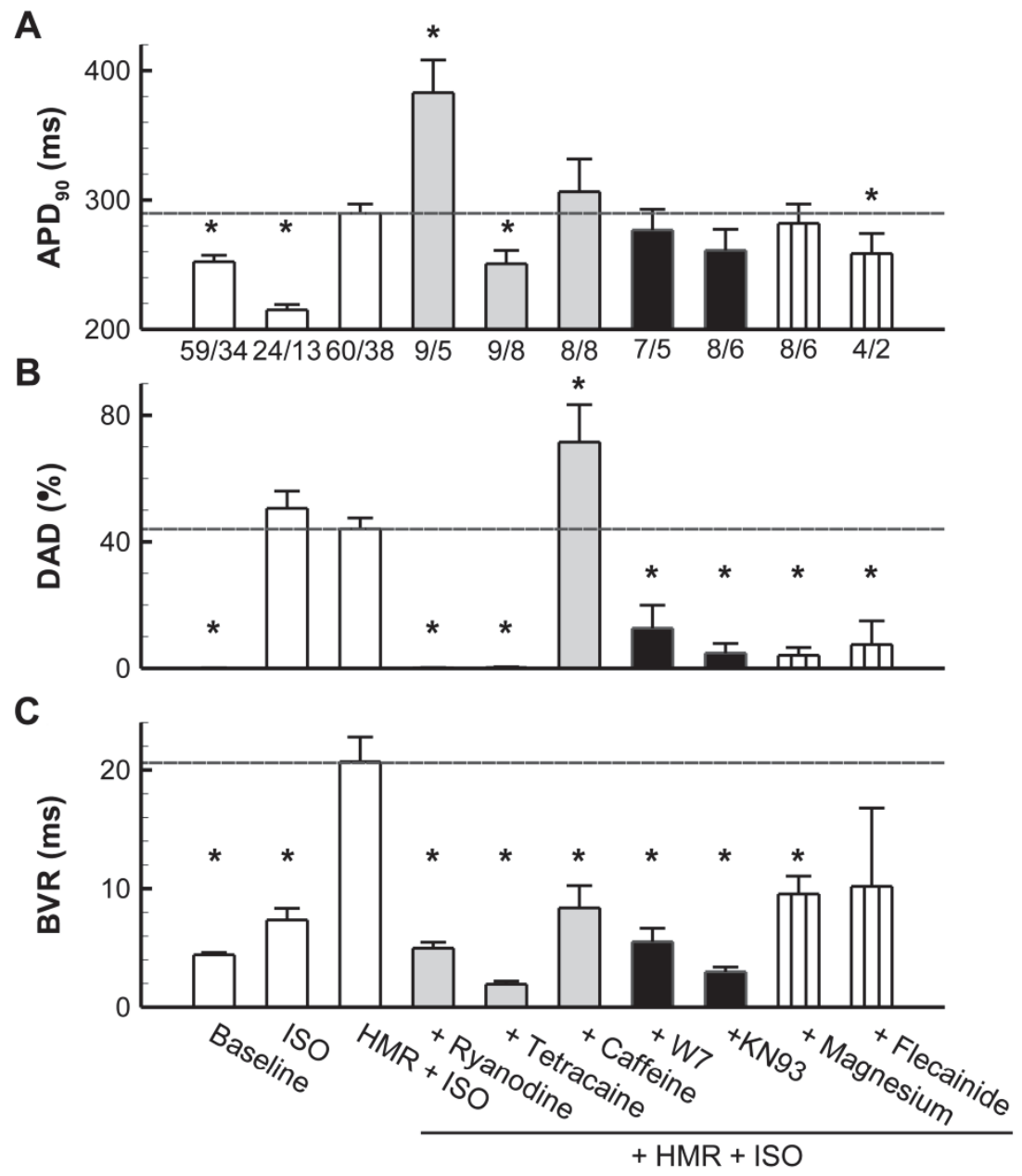

Figure 8: Interventions that abolish or homogenize SCR lower BVR. A. APD $D_{90} . n_{\text {cells }} / n_{\text {dogs }}$ are indicated below each bar. B. DAD occurrence. C. BVR at CL $=1000 \mathrm{~ms}$ at baseline, in the presence of ISO, HMR1556 + ISO, or HMR1556 + ISO combined with interventions to modulate SCR. Interventions are grouped by type (RyR modulation, grey; Calmodulin/CaMKII modulation, black; or agents also used clinically; striped). All interventions except caffeine result in a significant lowering of DAD incidence and a concomitant reduction of BVR, with increased (ryanodine), decreased (tetracaine, flecainide $(6 \mu \mathrm{mol} / \mathrm{L})$ ), or unaltered (W7 (1 $\left.\mu \mathrm{mol} / \mathrm{L}), \mathrm{KN} 93(5 \mu \mathrm{mol} / \mathrm{L}),\left[\mathrm{Mg}^{+}\right](5 \mathrm{mmol} / \mathrm{L})\right)$ APD ${ }_{90}$. Caffeine (maximally $500 \mu \mathrm{mol} / \mathrm{L}$ ) regularizes SCR occurrence, increasing DAD occurrence but lowering BVR without changing $\mathrm{APD}_{90}$ * * $\mathrm{P}<0.05$ versus HMR1556 + ISO.

Several studies have shown that $\mathrm{Ca}^{2+} / \mathrm{CaMKII}$ is an important signaling molecule affecting $\mathrm{Ca}^{2+}$-induced $\mathrm{Ca}^{2+}$ release (CICR) and that its inhibition can be antiarrhythmic ${ }^{24}$. Curran et al. ${ }^{25}$ demonstrated that SR $\mathrm{Ca}^{2+}$ leak during $\beta A R$ stimulation is mediated by CaMKII. In our experiments, inhibition of calmodulin using W7 reduced DAD incidence in all cells (from $44 \%$ to $8 \%$ at $1000 \mathrm{~ms} \mathrm{CL}, \mathrm{P}<0.05$ ) and completely abolished them in 3/7 cells. The decrease in DAD occurrence was paralleled by a decrease in BVR. Average APD was unaltered by W7, and systolic contractions remained intact. Application of the CaMKII inhibitor KN93 led to similar results (Figure $\mathbf{8}$, black bars). 
$\mathrm{Mg}^{2+}$ has been shown to suppress both EADs and DADs in different models ${ }^{26,27}$ and is used clinically as a first-line agent against TdP. Increasing extracellular $\left[\mathrm{Mg}^{2+}\right]$ from $1 \mathrm{mmol} / \mathrm{L}$ to $5 \mathrm{mmol} / \mathrm{L}$ abolished DADs induced after HMR1556 and ISO in 4/7 cells. In the remaining 3 cells, DAD incidence was significantly reduced. A concomitant reduction in BVR was seen. APDs were not significantly altered, and although systolic contractions were slightly reduced compared to beats that did not show SCR events before $\mathrm{Mg}^{2+}$, they remained stable, suggesting that the SR content remained constant (not shown). Similar results (albeit with reduced APD) were obtained with the class-IC antiarrhythmic agent flecainide (Figure 8, striped bars), which has been shown to be effective in preventing catecholaminergic polymorphic VT due to its effects on RyR and $\mathrm{Na}^{+}$channels ${ }^{28}$.

\subsubsection{Increases in $\left[\mathrm{Ca}^{2+}\right]_{0}$ Can Reinstate SCR Only When (Residual) RyR Function is Available}

We hypothesized that increases in $\left[\mathrm{Ca}^{2+}\right]_{0}$ after interventions that reduced DAD occurrence would lead to recurrence of SCR, DADs and increase BVR. Therefore, we raised the $\left[\mathrm{Ca}^{2+}\right]_{0}$ from $1.8 \mathrm{mmol} / \mathrm{L}$ to $3.6 \mathrm{mmol} / \mathrm{L}$ after application of ryanodine, tetracaine, or W7. This led to a reinduction of DADs after tetracaine or W7, and a significant decrease of APD (Figure 9A - C). However, raising $\left[\mathrm{Ca}^{2+}\right]_{0}$ after ryanodine did not lead to DADs, nor did it alter BVR, indicating that RyR function is required for SCR and without this BVR cannot be altered. Interestingly, BVR was not significantly reincreased after W7 and high $\left[\mathrm{Ca}^{2+}\right]_{0}$, whereas after tetracaine and high $\left[\mathrm{Ca}^{2+}\right]_{0}$ it was, despite DADs being reinstated under both conditions (Figure 9B, C). In the presence of W7, APs following SCR were no longer prolonged (Figure 9C, inset). These data suggest that calmodulin inhibition has a direct effect on the coupling between SCR and APD, possibly via $\mathrm{I}_{\text {CaL }}$. 

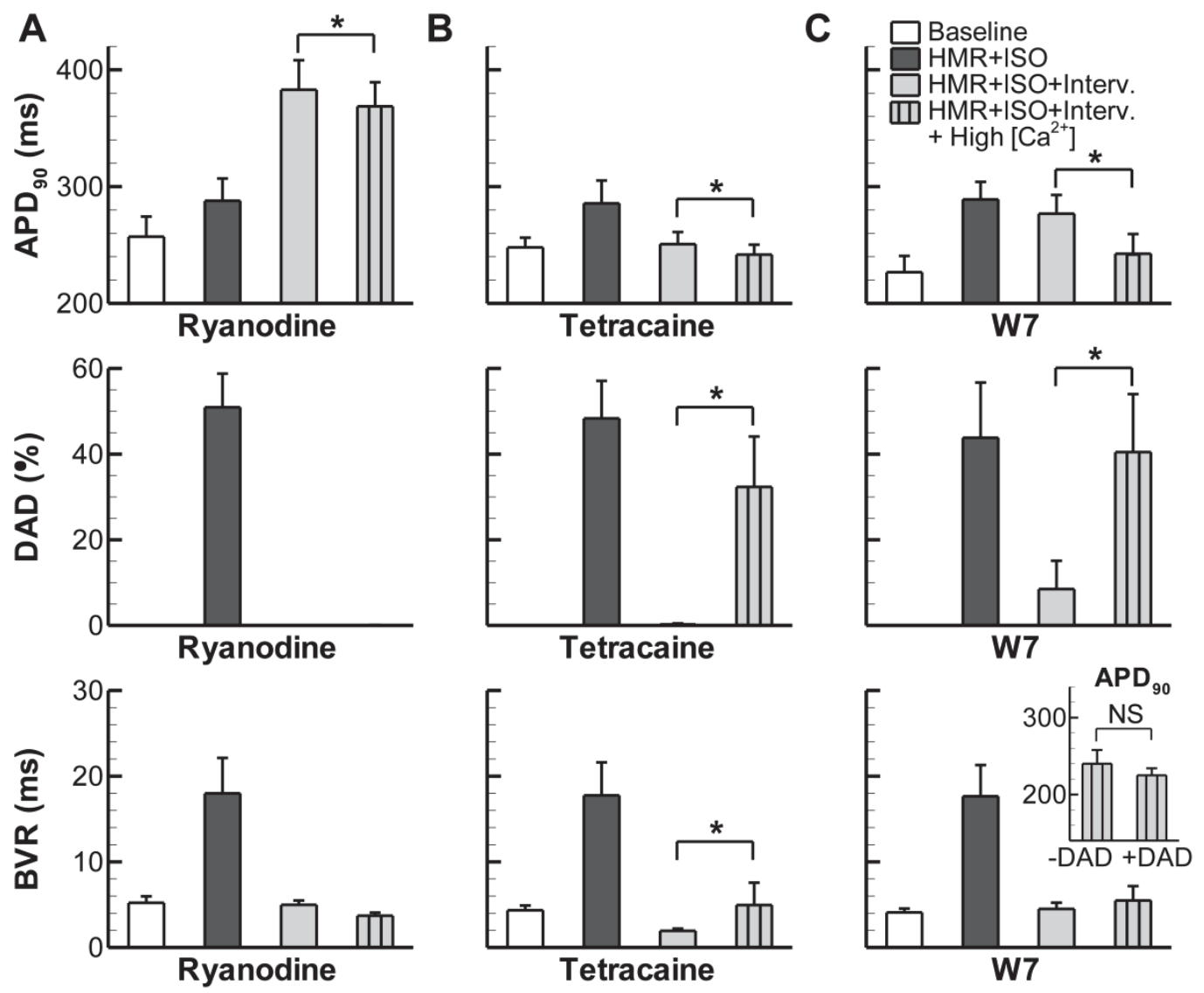

Figure 9: Effect of increased $\left[\mathrm{Ca}^{2+}\right]_{0}$ on $\mathrm{APD}_{90}$ (top panels), DAD occurence (middle panels), and BVR (bottom panels) under baseline conditions, HMR1556 + ISO, HMR1556 + ISO + Intervention or HMR1556 + ISO + Intervention + $3.6 \mathrm{mmol} / \mathrm{L}\left[\mathrm{Ca}^{2+}\right]_{0}$. A. Ryanodine. B. Tetracaine. C. W7. * indicates $\mathrm{P}<0.05$. Only significance of intervention versus intervention in the presence of high $\left[\mathrm{Ca}^{2+}\right]_{0}$ is indicated for clarity reasons. Inset in lower-right panel shows $\mathrm{APD}_{90}$ in the presence of HMR1556 + ISO + W7 + increased $\left[\mathrm{Ca}^{2+}\right]_{0}$ for beats in the absence or presence of a preceding DAD.

\subsubsection{Reduced $\mathrm{Ca}^{2+}$-Dependent I nactivation of I $\mathrm{CaL}_{\text {UL }}$ Underlies APD Prolongation After SCR}

We employed a computational model of the canine ventricular myocyte to further investigate the ionic basis of the coupling between SCR and APD prolongation. After pacing to steady state, a partial release of $\mathrm{SR} \mathrm{Ca}^{2+}$ was induced during diastole. After this release, APD was prolonged, consistent with experimental observations (Figure 10A; 1000-ms CL in the presence of ISO). The prolonged APD was associated with a decreased CaT, an increase in the sustained component of $\mathrm{I}_{\mathrm{CaL}}$ and increased $\mathrm{I}_{\mathrm{Ks}}$. The increase in $\mathrm{I}_{\mathrm{Ks}}$ was due to the increased plateau potential resulting from the larger $\mathrm{I}_{\mathrm{Ca}}$ and counteracted the repolarization delay. As such, APD prolongation was significantly larger when $I_{K_{s}}$ was inhibited (Figure 10B). An increase in plateau $V_{m}$ was also observed in experimental recordings (maximum amplitude of plateau was $114 \pm 1 \mathrm{mV}$ without prior $\mathrm{DAD}$ and $118 \pm 1 \mathrm{mV}$ following DADs at $1000 \mathrm{~ms} \mathrm{CL}$ in the presence of HMR1556 + ISO, P<0.05). 
Several currents underlying the ventricular AP are modified by intracellular $\mathrm{Ca}^{2+}$. To determine the relative contribution of each component, we used the model to selectively inhibit each current for one final beat at steady state following either a normal diastole or SCR. Inhibition of the main currents $\left(\mathrm{I}_{\mathrm{NaCa}}\right.$ and $\mathrm{I}_{\mathrm{Cl}(\mathrm{Ca})}{ }^{3,}{ }^{29}$ ) only had a minor impact on the APD differences in the presence and absence of SCR (Figure $10 \mathrm{C}$ ). In contrast, inhibition of $\mathrm{I}_{\mathrm{CaL}}$ or RyR was able to significantly reduce the DAD-provoked APD prolongation. More specifically, when only $\mathrm{Ca}^{2+}$-dependent inactivation (CDI) of $\mathrm{I}_{\mathrm{CaL}}$ was inhibited, APD after SCR was no longer prolonged compared to APD without prior SCR (Figure 10C). Further investigation showed any differences in APD in these conditions were due to 'priming' of CDI due to a transition of L-type $\mathrm{Ca}^{2+}$ channels to the $\mathrm{Ca}^{2+}$-dependent tier of the Markov model induced by the $\mathrm{Ca}^{2+}$ that was released during the SCR, prior to activation of inhibition.

Combined, our data implicate reduced $\mathrm{SR} \mathrm{Ca}^{2+}$-release-dependent inactivation of $\mathrm{I}_{\mathrm{CaL}}$ as the mechanism underlying APD prolongation after SCR. This prediction was confirmed under voltage-clamp conditions in native canine ventricular myocytes when the extracellular solution was modified to isolate $\mathrm{I}_{\text {CaL }}$ (Figure 11). 

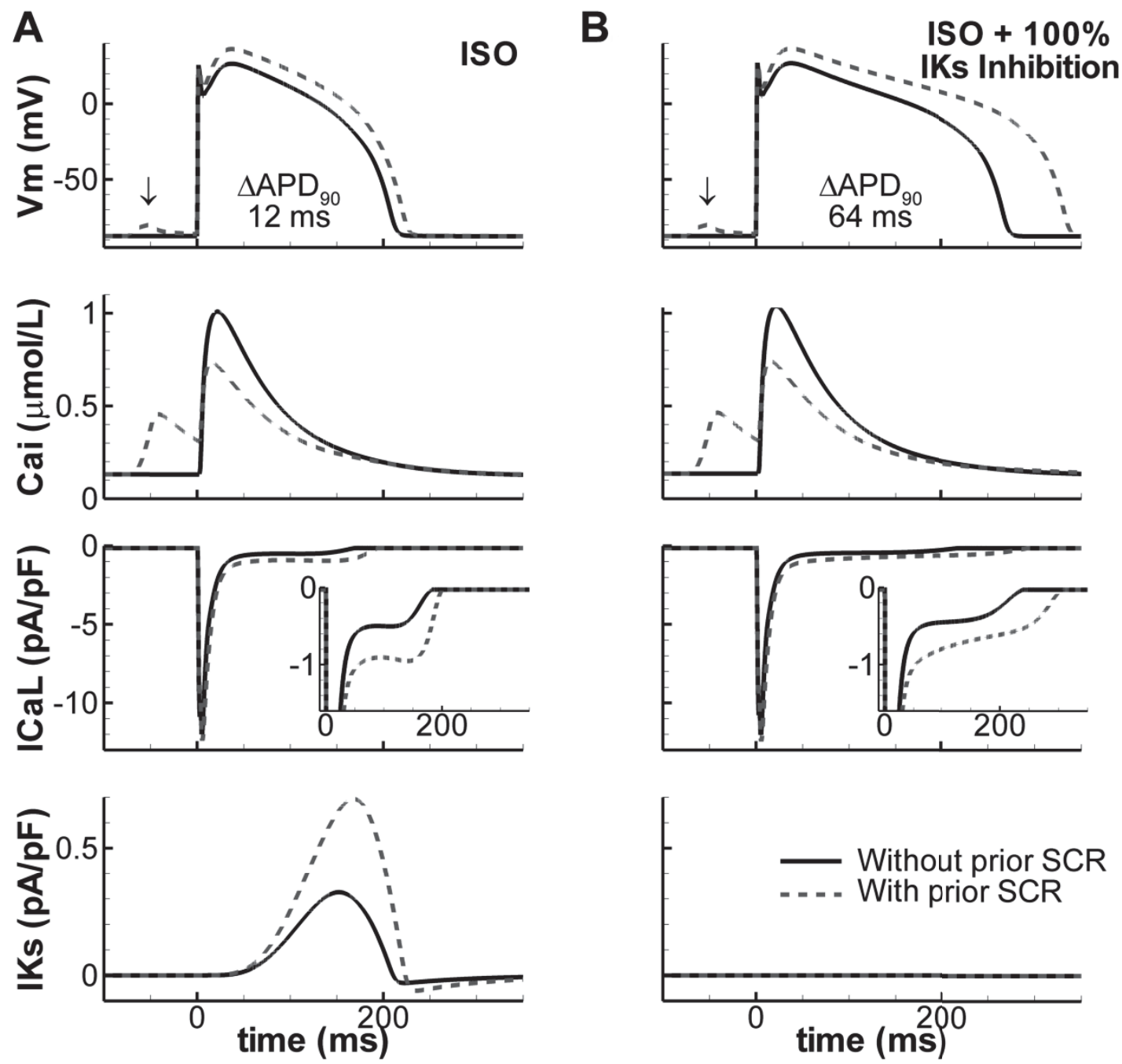

C

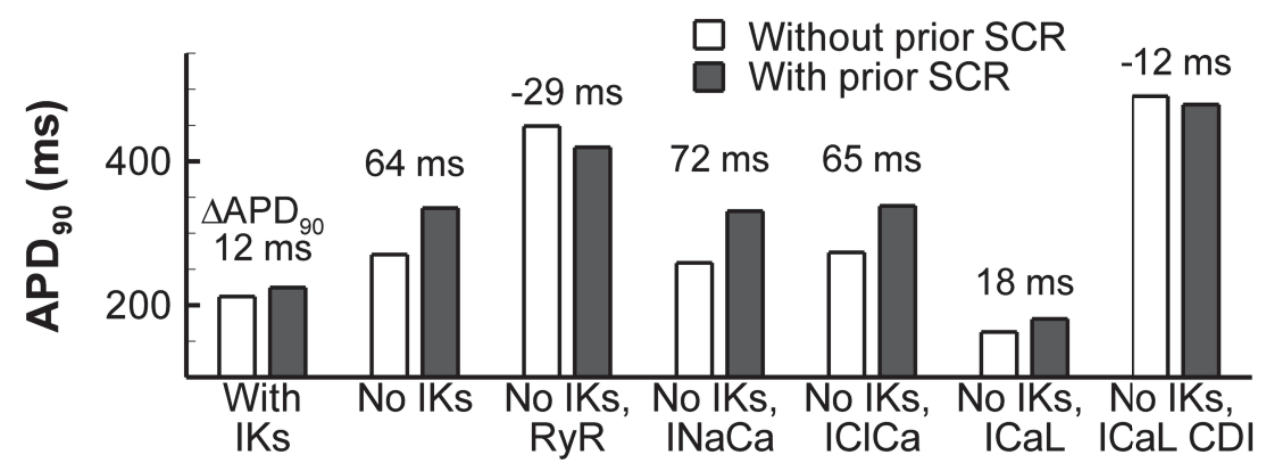

Figure 10: Computational model of the canine ventricular myocyte implicates reduced SR $\mathrm{Ca}^{2+}$-dependent inactivation of $\mathrm{I}_{\mathrm{CaL}}$ to cause APD prolongation. A. AP, intracellular $\mathrm{Ca}^{2+}, \mathrm{I}_{\mathrm{CaL}}$ and $\mathrm{I}_{\mathrm{KS}}$ (top to bottom) with (dashed lined) or without (solid lines) prior SR Ca ${ }^{2+}$ release for a single beat after pacing to steady state in the presence of ISO at CL of $1000 \mathrm{~ms}$. The DAD resulting from the SCR is indicated by an arrow. Inset in $3^{\text {rd }}$ panel shows $\mathrm{I}_{\mathrm{CaL}}$ during AP plateau on an expanded scale. B. Similar to panel A in the presence of ISO and complete inhibition of $\mathrm{I}_{\mathrm{Ks}}$. C. After pacing to steady state in the presence of ISO, SCR was triggered (shaded bars) or not (white bars) and individual currents/fluxes were blocked for one final beat. $A P D_{90}$ differences are indicated above each bar. Only inhibition of $\mathrm{SR} \mathrm{Ca}^{2+}$ release (RyR) or $\mathrm{I}_{\mathrm{CaL}} \mathrm{CDI}$ was sufficient to completely abolish the APD prolongation after SCR. 


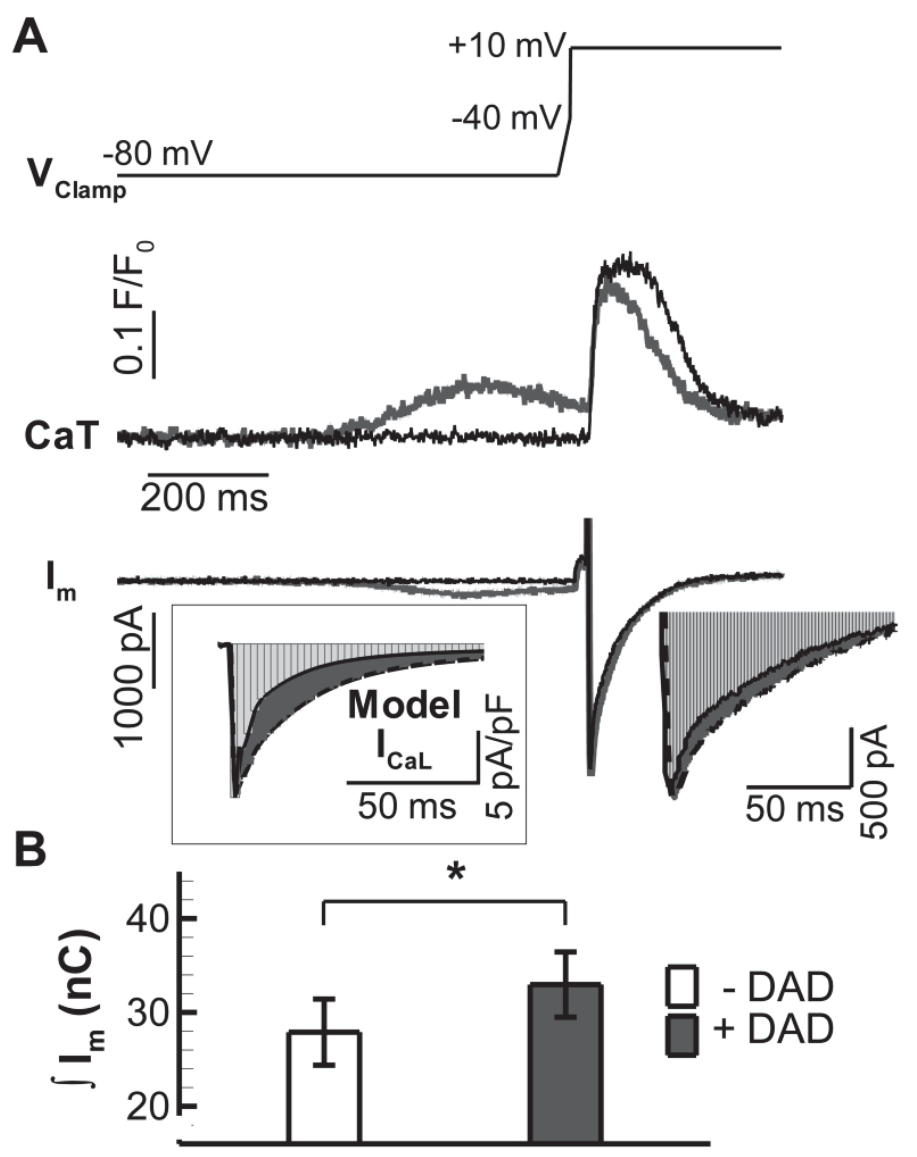

Figure 11: $\mathrm{I}_{\mathrm{CaL}}$ inactivation is slowed after SCR. A. Voltage-clamp protocol (top panel), intracellular $\mathrm{Ca}^{2+}$ (middle panel) and membrane current (bottom panel) in a representative canine ventricular myocyte in the presence of HMR1556 + ISO + increased $\left[\mathrm{Ca}^{2+}\right]_{0}$ (3.6 or 5.0 $\mathrm{mmol} / \mathrm{L}$ ) in the absence (black) or presence of prior SCR (grey). Right inset shows membrane current at an expanded scale. 4-AP $(5.0 \mathrm{mmol} / \mathrm{L})$ and $\mathrm{BaCl}_{2}(0.1 \mathrm{mmol} / \mathrm{L})$ were used to isolate $\mathrm{I}_{\mathrm{CaL}}$. Left inset shows model results under similar conditions. B. Quantification of average membrane current integral (shaded area in right inset of panel A) in 6 canine ventricular myocytes in the absence or presence of SCR. Prior SCR significantly increases total inward current $(*: \mathrm{P}<0.05)$.

During a voltage step to $+10 \mathrm{mV}$ from a holding potential of $-80 \mathrm{mV}$ a significant increase in the integral of $\mathrm{I}_{\mathrm{CaL}}$ was observed after SCR: $\int \mathrm{I}_{\mathrm{CaL}}=27.9 \pm 3.5 \mathrm{nC}$ vs $33.0 \pm 3.5 \mathrm{nC}$ in the absence or presence of SCR, respectively $(\mathrm{P}<0.05)$.

\subsubsection{Relation Between Aftercontractions, Repolarization Prolongation and Dispersion, and Arrhythmogenesis in the I ntact Canine Heart}

To evaluate whether the single-cell mechanisms described could affect arrhythmogenesis in-vivo, we employed a canine model of drug-induced LQT1 ${ }^{11}$. Application of a bolus of ISO during continuous HMR1556 infusion resulted in aftercontractions exclusively in the LVP signal prior to the development of ventricular premature beats and TdP (Figure 12A). In parallel with the occurrence of aftercontractions, we observed paradoxical QT prolongation during $\beta A R$ heart-rate 
acceleration, resulting in a significant increase of the QT interval in the last sinus beats prior to the extrasystoles triggering TdP (Figure 12B). Similarly, LV MAP duration was significantly prolonged in these beats and a significant increase in $\mathrm{T}_{\text {peak }}-\mathrm{T}_{\text {end }}$ interval was noted (Figure 12B), which could reflect an increased dispersion of repolarization.

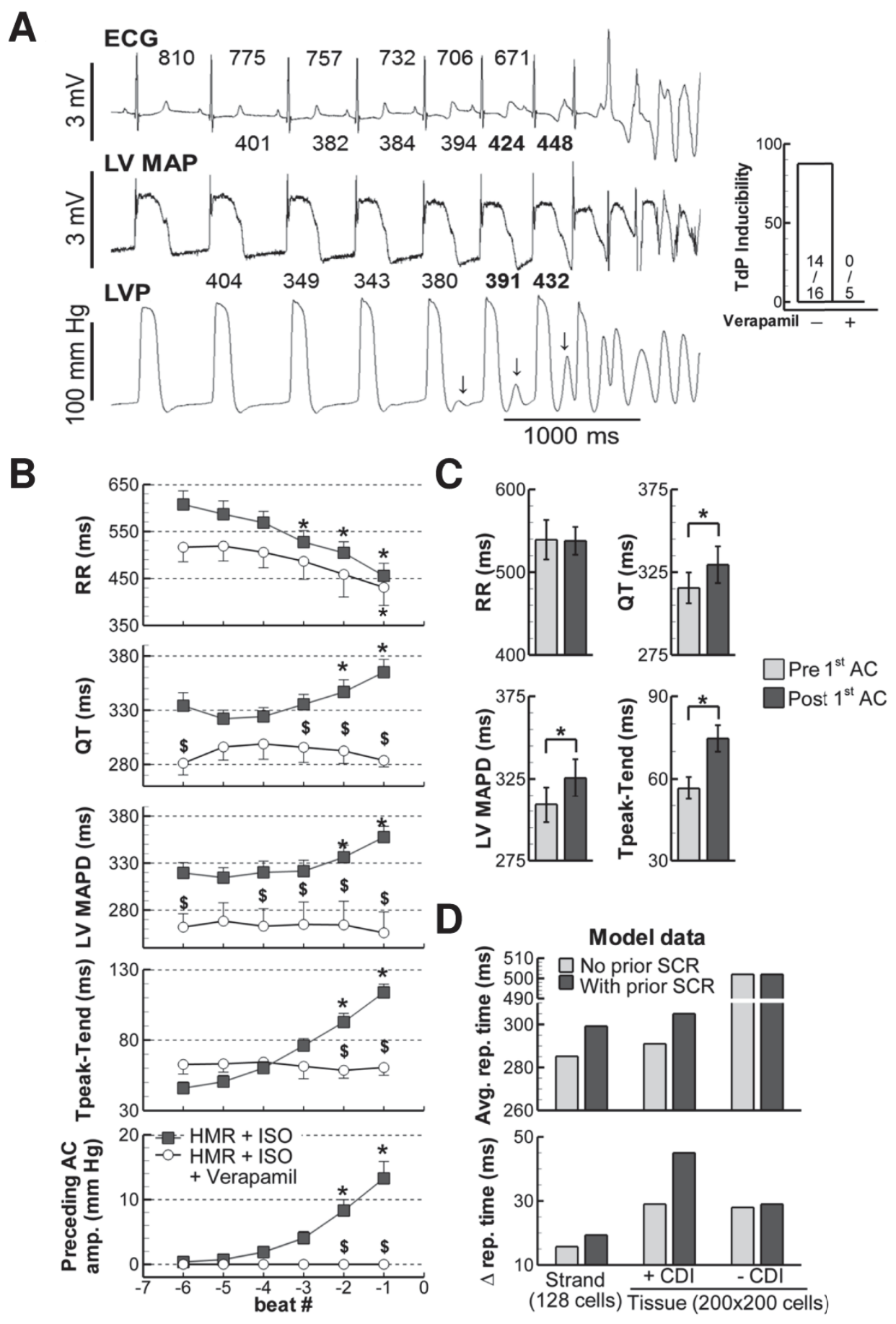

Figure 12: Aftercontractions and repolarization prolongation precede the occurrence of TdP in an in-vivo canine model of LQT1. 
A. ECG, LV MAPs and LVP in the 6 beats prior to the first extrasystolic beat and initiation of $\mathrm{TdP}$ in a representative experiment after a bolus of ISO in the presence of HMR1556. Inset shows TdP inducibility by ISO during HMR1556 in the absence or presence of verapamil. B. Group data showing electrophysiological properties (RR interval, QT interval, LV MAP duration and $T_{\text {peak }}-T_{\text {end }}$ interval) and average amplitude of the preceding aftercontraction in the absence (filled squares; $n=14$ ) or presence (open circles; $n=5$ ) of verapamil. * indicates $\mathrm{P}<0.05$ compared to beat -6 (ANOVA for repeated measures with Bonferroni post-hoc test); $\$$ indicates $P<0.05$ versus the absence of verapamil for the same beat. Data are shown relative to the start of TdP or during the maximal chronotropic response to ISO. C. Comparison of the electrophysiological properties of the beats preceding (light grey) and following (dark grey) the first aftercontraction during HMR+ISO: RR interval, QT interval, LV MAP duration, $\mathrm{T}_{\text {peak }} \mathrm{T}_{\text {end }}$ interval. *; $\mathrm{P}<0.05$ compared to pre 1st AC. D. Effect of diastolic $\mathrm{Ca}^{2+}$ release in $25 \%$ of the cells on average repolarization time (top) and spatial dispersion of repolarization (bottom) in 1-dimensional strand simulations and 2-dimensional tissue simulations with intact or disabled $\mathrm{I}_{\mathrm{CaL}} \mathrm{CDI}$.

We focused on repolarization duration by comparing the beats preceding and following the first notable aftercontraction. QT, LV MAP and $T_{\text {peak }}-T_{\text {end }}$ durations were significantly increased in the beat after the first aftercontraction, whereas the RR intervals were unchanged (Figure 12C). Furthermore, the $\mathrm{I}_{\mathrm{CaL}}$ inhibitor verapamil ( $0.4 \mathrm{mg} / \mathrm{kg}$ ) prevented TdP induction by ISO during continuous HMR1556 infusion in 5/5 animals (Figure 12A), although some ventricular extrasystolic activity could still be observed (Figure 13). Similar chronotropic responses were seen after ISO in the presence of verapamil but, in the absence of aftercontractions, QT, LV MAP and $T_{\text {peak }}-T_{\text {end }}$ prolongation were significantly smaller when compared to HMR1556 and ISO alone (Figure 12B). These data illustrate the role of $\mathrm{Ca}^{2+}$-dependent regional repolarization prolongation for TdP induction in this model.

To further test the hypothesis that SCR at the single-myocyte level can contribute to the QT and $\mathrm{T}_{\text {peak }}-\mathrm{T}_{\text {end }}$ interval prolongation seen in-vivo, we performed computer simulations of both a homogeneous one-dimensional strand and a two-dimensional tissue of electrically-coupled myocytes. Consistent with our hypothesis, diastolic SR $\mathrm{Ca}^{2+}$ release increased repolarization duration and spatial dispersion of repolarization in both simulations (Figures 12D, 14 and 15), and this effect was completely abolished by inhibition of $\mathrm{I}_{\mathrm{CaL}}$ CDI (Figure 12D and 16). 
A
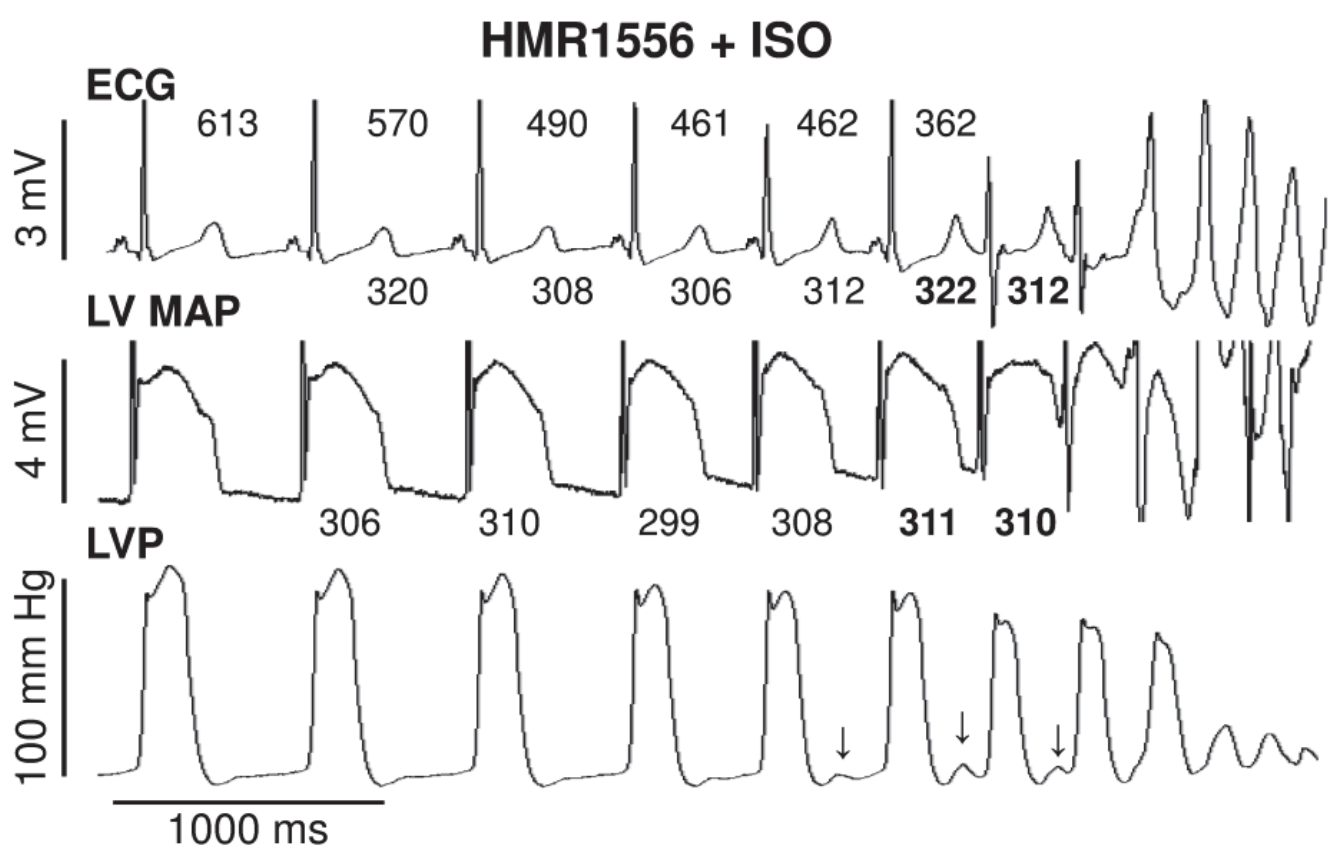

B
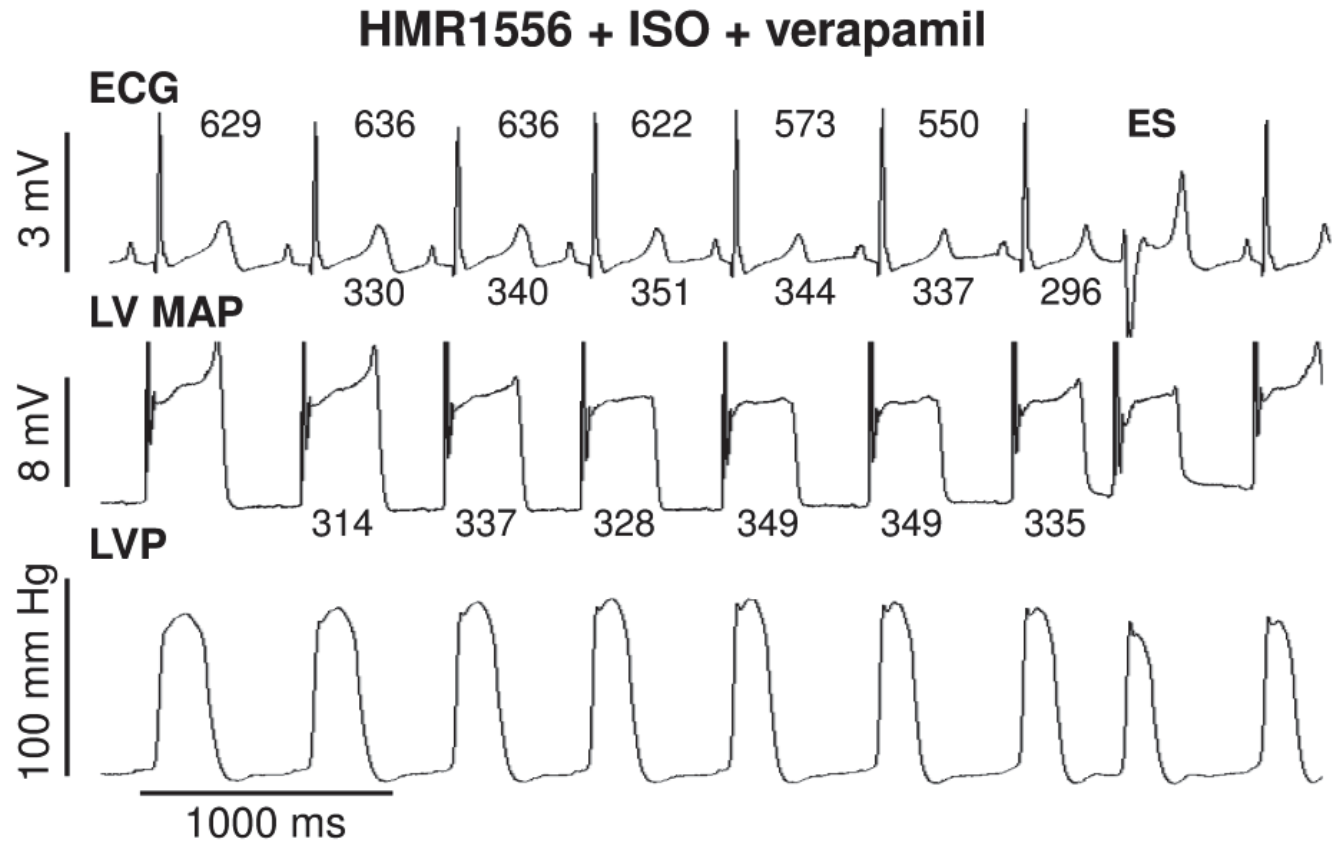

Figure 13: A. ECG, left-ventricular (LV) monophasic action potential (MAP) and LV pressure (LVP) recordings following a bolus of ISO during continuous HMR1556 infusion in the absence or B. presence (of verapamil $(0.4 \mathrm{mg} / \mathrm{kg})$ in the same dog. RR intervals are indicated above the ECG traces and QT and LV MAP durations are indicated below the ECG and LV MAP signals, respectively. TdP was induced in the absence of verapamil and was preceded by aftercontractions in the LVP signal (indicated by arrows). Verapamil reduced the systolic LVP by approximately $17 \%$ and prevented the aftercontraction formation and TdP induction. 
A
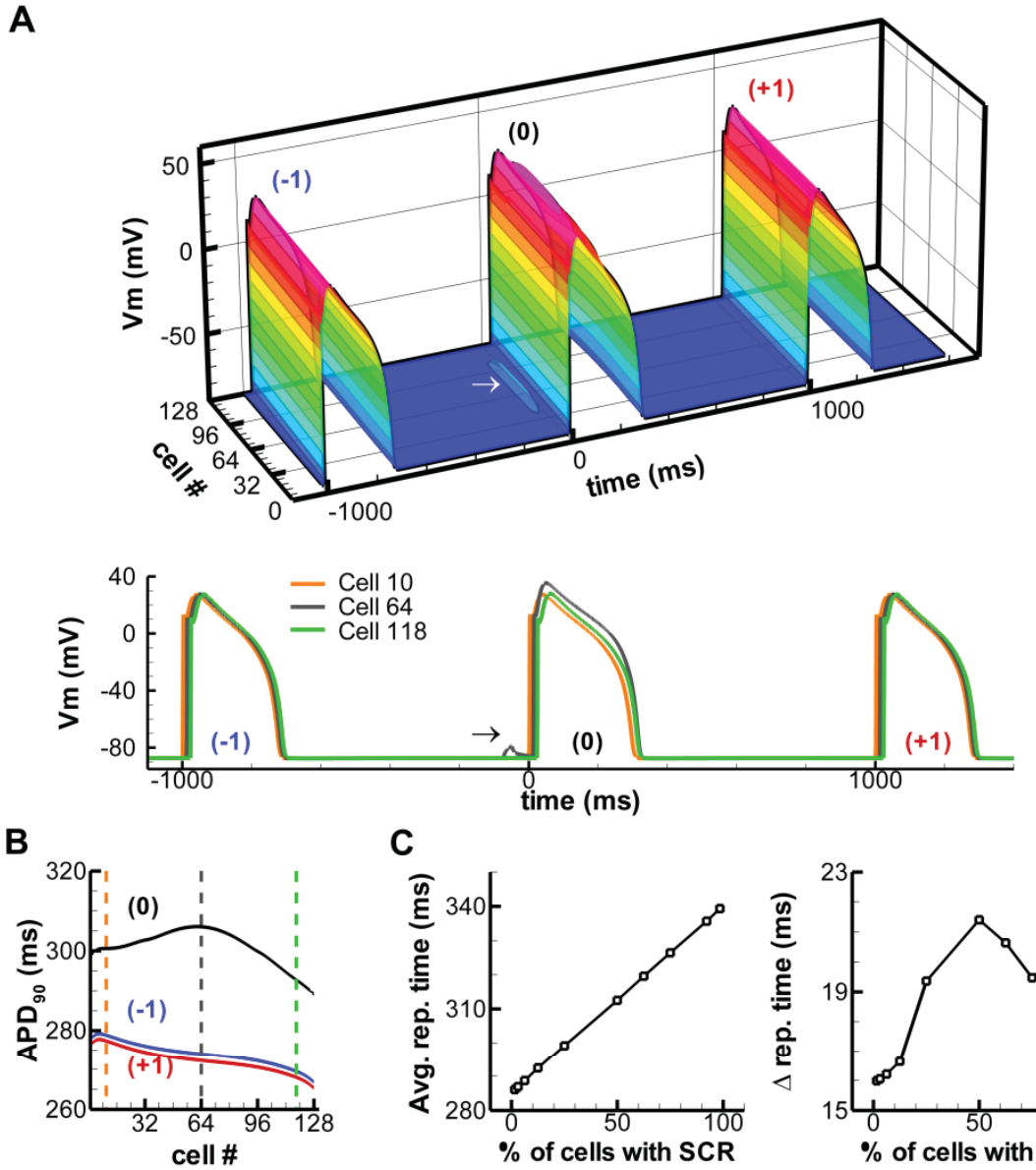

C
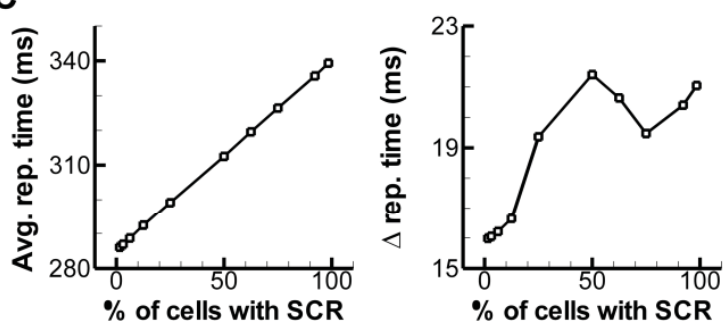

Figure 14: Multicellular simulations in a one-dimensional strand of 128 cells in the presence of ISO and $\mathrm{I}_{\mathrm{KS}}$ blockade. A. APs during steady state pacing at $\mathrm{CL}=1000 \mathrm{~ms}$ (top panel). Prior to beat (0), diastolic SR $\mathrm{Ca}^{2+}$ release was initiated in cells 32-96 (arrow). The bottom panel shows an overlay of Vm for cell 10 (orange, no DAD), cell 64 (grey, with DAD indicated by arrow) and cell 118 (green, no DAD) for these three beats. B. APD 90 for all cells in the strand for these 3 beats. Cells 10, 64 and 118 have been indicated by vertical dashed lines. The presence of a preceding diastolic $\mathrm{SR} \mathrm{Ca}{ }^{2+}$ release causes a pronounced prolongation of $\mathrm{APD}_{90}$ of beat ( 0 ) throughout the strand (black line). In addition, an increase in the spatial dispersion of repolarization could be observed. $\mathbf{C}$. Average repolarization time (activation time $+\mathrm{APD}_{90}$; left panel) and spatial dispersion of repolarization (maximum repolarization time minimum repolarization time; right panel) as a function of the number of cells with diastolic $\mathrm{SR} \mathrm{Ca}{ }^{2+}$ release (SCR). SCR caused a progressive increase in the average repolarization time. Dispersion of repolarization was influenced by the number of cells with SCR (with a maximum around $50 \%$ ). A low spatial dispersion of repolarization was expected at $100 \%$, when all cells exhibit SCR. However, spatial dispersion of repolarization also depends on the timing of the SCR. Since SCR was initiated at the same time in all cells, but the time of $\mathrm{Ca}^{2+}$-induced $\mathrm{Ca}^{2+}$ release differs due to action potential propagation along the strand, there is a longer time for SR refilling after the SCR in cell 128 than in cell 1 . As such, the amount of APD prolongation differs throughout the strand resulting in an increased spatial dispersion even at $100 \%$. Spatial dispersion of repolarization is a complex, non-monotonic function that depends on the number of cells with SCR, their location in the strand, the timing of the SCR and the conduction velocity. 
A

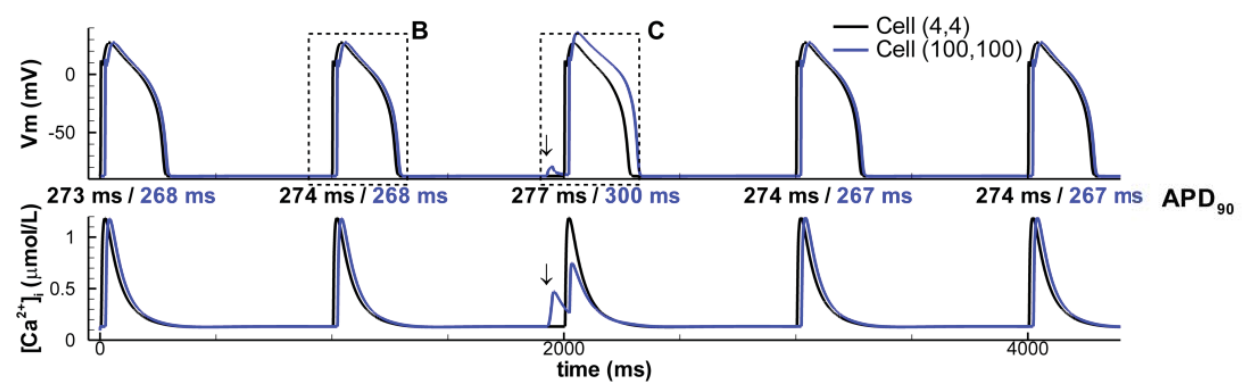

B
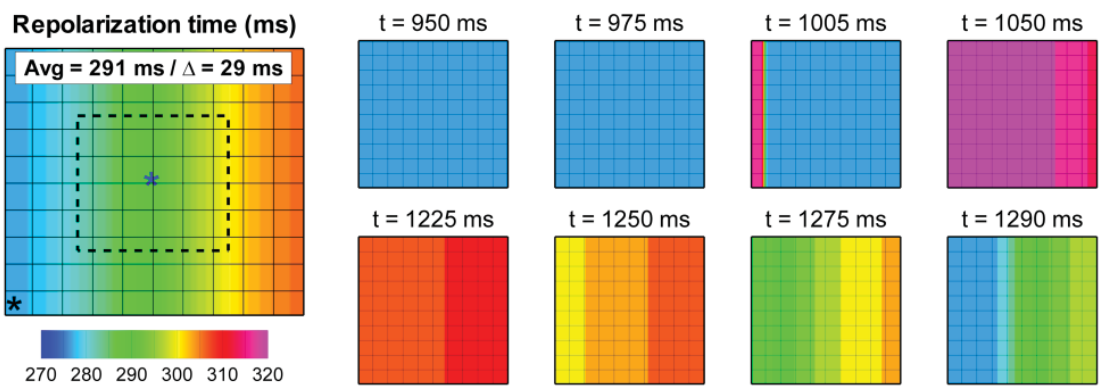

$\mathrm{Vm}(\mathrm{mV})$

$t=1225 \mathrm{~ms}$

$\mathrm{t}=1250 \mathrm{~ms}$
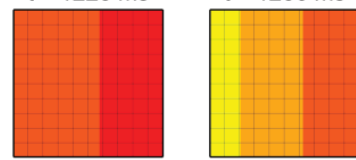

$\mathrm{t}=1275 \mathrm{~ms}$

$t=1290 \mathrm{~ms}$
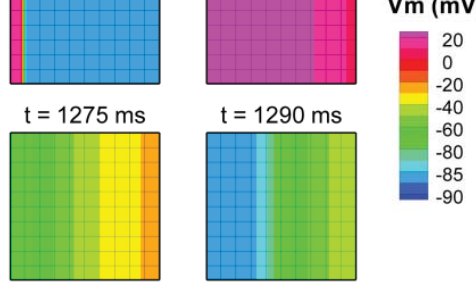

C Repolarization time (ms)

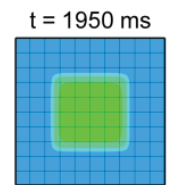

$\mathrm{t}=2225 \mathrm{~ms}$

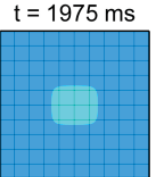

$\mathrm{t}=2250 \mathrm{~ms}$

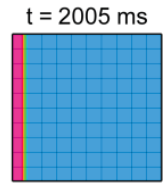

$\mathrm{t}=2275 \mathrm{~ms}$
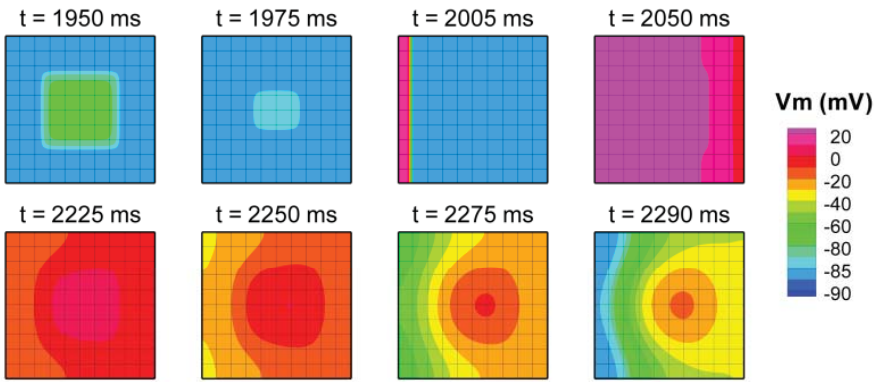

Figure 15: Multicellular simulations in homogeneous two-dimensional tissue of $200 \times 200$ nodes in the presence of ISO and $I_{\mathrm{Ks}}$ blockade. A. APs (top panel) and $\left[\mathrm{Ca}^{2+}\right] \mathrm{i}$ (bottom panel) for 5 beats during steady-state pacing at $1000-\mathrm{ms} \mathrm{CL}$ for a cell on the edge of the tissue (at coordinates $(4,4)$, black lines) and a cell in the center of the tissue (blue lines). The location of these cells is indicated with stars in panels B and C (left). APD90 of each AP is given below each beat in the corresponding color. Diastolic SR $\mathrm{Ca}^{2+}$ release was induced from 1925 to $1950 \mathrm{~ms}$ (indicated by arrows) for a region of $100 \times 100$ cells ( $25 \%$ of the cells) around the center of the tissue (i.e., cells with $\mathrm{x}$ and $\mathrm{y}$ coordinates between 50 and 150, dashed box in panels B and C). B. Spatial electrophysiological properties of beat 2 . The left panel shows repolarization time (activation time + APD90) at every point in the tissue. Average repolarization time was $291 \mathrm{~ms}$ and spatial dispersion of repolarization (maximum-minimum repolarization time) was $29 \mathrm{~ms}$. The eight smaller panels on the right show voltage maps at different time instants. The voltage maps show a homogeneous diastolic interval followed by a planar wave propagating from left to right following pacing (conduction velocity of $49 \mathrm{~cm} / \mathrm{s}$ ). Repolarization occurs uniformly around $1290 \mathrm{~ms}$. C. Similar to panel B for beat 3 which is preceded by diastolic $\mathrm{Ca}^{2+}$ release in a subset of the cells. Average repolarization time is longer (305 ms) and shows increased spatial dispersion (45 ms), in line with one-dimensional simulations and in-vivo experimental data. Voltage maps identify the DAD in the center of the tissue and the prolonged repolarization of this same area during the following beat. 


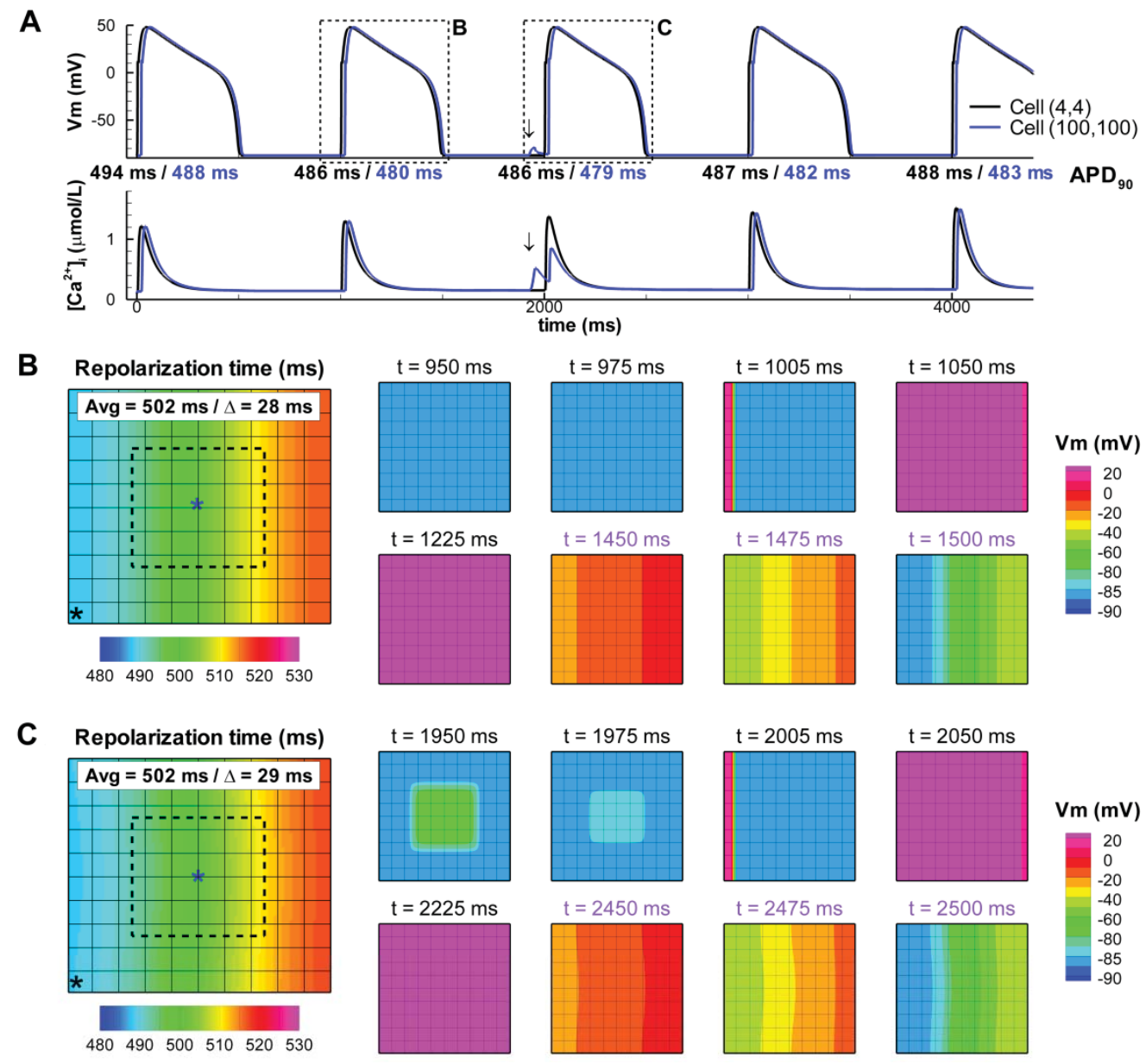

Figure 16: Multicellular simulations in homogeneous two-dimensional tissue of 200x200 nodes in the presence of ISO and $\mathrm{I}_{\mathrm{Ks}}$ blockade after inhibition of $\mathrm{Ca}^{2+}$-dependent inactivation (CDI) of $\mathrm{I}_{\mathrm{CaL}}$. Simulation protocol and figure layout are identical to Figure 15, except for the inhibition of CDI for these 5 beats (after pacing to steady-state in the presence of ISO and $\mathrm{I}_{\mathrm{Ks}}$ blockade). Inhibiting CDI prolongs APD90, consistent with experimental data ${ }^{30}$ and single-cell simulations (Figure 10). Inhibition of CDI prevents APD prolongation following diastolic $\mathrm{SR} \mathrm{Ca}^{2+}$ release despite similar DAD and systolic $\mathrm{Ca}^{2+}$ transient properties and prevents increased spatial dispersion of repolarization. Note that due to this APD prolongation different time-points were used for the voltage maps in panels B and C (indicated in purple). 


\subsection{Discussion}

In this study, we elucidated the relationship between SCR, AP prolongation and BVR in canine LV myocytes and provide arguments for its arrhythmogenic significance in the in-vivo beating heart. Our data indicate that under conditions of ISO-induced $\mathrm{Ca}^{2+}$ loading, SCR can occur over a wide range of pacing CLs and that APD following SCR is significantly prolonged. Reduced CICR-dependent inactivation of $\mathrm{I}_{\mathrm{CaL}}$ after SCR is involved in this AP prolongation. The increase in BVR was strongly dependent on the degree of APD prolongation after SCR. In anesthetized dogs subjected to similar conditions, we observed the occurrence of mounting aftercontractions in parallel with $\mathrm{QT}, \mathrm{T}_{\text {peak }}-\mathrm{T}_{\text {end }}$ and LV MAP prolongation just prior to TdP which could be prevented by inhibition of $\mathrm{I}_{\mathrm{CaL}}$ and $\mathrm{Ca}^{2+}$ load with verapamil. SCR-related repolarization prolongation was reproduced in multicellular computer simulations. Our data align with recent data showing that local $\beta A R$ stimulation synchronizes SCR in the normal rabbit heart, leading to $\mathrm{Ca}^{2+}$-mediated focal arrhythmia ${ }^{31}$. In the setting of exaggerated spatio-temporal dispersion of repolarization such focal activity may trigger TdP, as actually observed in the canine model of drug-induced LQT1 syndrome and $\beta A R$ stimulation (see also ${ }^{11}$ ).

Previous cellular studies ${ }^{32}-{ }^{34}$ have shown that large CaTs result in abbreviation of APD, whereas a small CaT after SR $\mathrm{Ca}^{2+}$ depletion corresponds to prolonged APD. In agreement, we find substantial APD prolongation after application of ryanodine due to reduced CDI of $\mathrm{I}_{\mathrm{CaL}}$. Furthermore $\mathrm{I}_{\mathrm{CaL}} \mathrm{CDI}$ is sensitive to (partial) $\mathrm{SR} \mathrm{Ca}^{2+}$ unloading by SCR. These changes in CDI are sufficient to modulate APD on a beatto-beat basis over a wide range of CLs. Interestingly, Spencer and Sham reported the opposite effect in guinea-pig ventricular myocytes perhaps due to species differences in the balance of NCX and $\mathrm{I}_{\mathrm{CaL}}{ }^{35}$. In the present study, we used $\mathrm{Ca}^{2+}$ sensitive fluorescent probes and cell shortening as $\mathrm{Ca}^{2+}$ indicators. Both measures reflect cytosolic $\mathrm{Ca}^{2+}$ levels, which are substantially different from the subsarcolemmal $\left[\mathrm{Ca}^{2+}\right]$ influencing $\mathrm{Ca}^{2+}$-activated membrane currents.

\subsubsection{The I mportance of $I_{\mathrm{Ks}}$ Blockade}

Burashnikov and Antzelevitch ${ }^{36}$ demonstrated that $\mathrm{I}_{\mathrm{Ks}}$ block alone was insufficient to induce DADs or modulate repolarization heterogeneity in canine transmural ventricular tissues ${ }^{36}$. However, it amplified the effects of adrenergic stimuli. At the myocyte level, we have previously shown that $\mathrm{I}_{\mathrm{Ks}}$ blockade (via KCNQ1 inhibition ${ }^{37}$ ) has minimal effects on APD and BVR under baseline conditions. During BAR stimulation and $\mathrm{I}_{\mathrm{KS}}$ blockade, BVR is significantly increased and this is at least partly dependent on $\left[\mathrm{Ca}^{2+}\right]_{i}{ }^{14}$. Here we extend this by determining an important role for CDI of $\mathrm{I}_{\mathrm{CaL}}$, at least in the presence of SCR. Under baseline conditions, $\mathrm{I}_{\mathrm{Kr}}$ is the main repolarizing current in canine ventricular myocytes and inhibition of $\mathrm{I}_{\mathrm{Kr}}$ can cause 
EADs due to APD prolongation and subsequent $\mathrm{I}_{\text {CaL }}$ reactivation ${ }^{14}$. During additional $\beta A R$ stimulation, the balance of the repolarization reserve is altered and the role of $\mathrm{I}_{\mathrm{Ks}}$ becomes more prominent; enhanced $\mathrm{I}_{\mathrm{Ks}}$ prevents repolarization instability and EAD generation by other proarrhythmic mechanisms (e.g., $\mathrm{I}_{\mathrm{Kr}}$ inhibition or augmentation of late $I_{\mathrm{Na}}$ ). In contrast, inhibition of $I_{\mathrm{Ks}}$ during $\beta A R$ stimulation leads to APD prolongation, increased BVR and EADs indicating that under our conditions with $\beta A R$ stimulation, $I_{K s}$ is a major contributor to repolarization. Barandi et al. ${ }^{38}$ have previously shown that the degree of APD prolongation induced by pharmacological block of repolarizing currents or augmentation of depolarizing currents, depends on baseline APD. Consistent with these results, we find a more pronounced APD prolongation after $\mathrm{SCR}$ at slow $\mathrm{CL}$ and in the presence of $\mathrm{I}_{\mathrm{Ks}}$ blockade. In our experiments, APD prolongation after SCR is particularly pronounced because the increase in $\mathrm{I}_{\mathrm{CaL}}$ also elevates the plateau potential which increases $\mathrm{I}_{\mathrm{Ks}}$ activation and offsets the prolongation induced by $\mathrm{I}_{\mathrm{CaL}}$ (Figure 10A, bottom panel). This compensating mechanism is absent when $I_{\mathrm{Ks}}$ is inhibited. Thus, $\mathrm{I}_{\mathrm{Ks}}$ inhibition exacerbates the effect of SCR on APD prolongation.

\subsubsection{The Role of SCR in Arrhythmogenesis}

It has long been established that SCR-induced triggered activity is a major arrhythmogenic mechanism during $\mathrm{Ca}^{2+}$ overload and its occurrence is increased in various pathological conditions ${ }^{6,39}$. Here we observed that diastolic SCR prolongs the subsequent APD, suggesting that even SCR below the threshold for triggered activity has important electrophysiological effects that may be proarrhythmic. Regional prolongation of APD after SCR in the intact heart may cause increased spatial dispersion of repolarization between regions with the highest $\mathrm{Ca}^{2+}$ load (generating $\mathrm{SCR}$ ) and regions with lower $\mathrm{Ca}^{2+}$ loading. In agreement with this hypothesis, we observed increased QT duration and $T_{\text {peak }}-T_{\text {end }}$ intervals following the first aftercontraction upon a challenge with a bolus of ISO during $\mathrm{I}_{\mathrm{Ks}}$ blockade in-vivo. Regional heterogeneities in ion-channel expression or intercellular conduction may further amplify this dispersion. Combined, these mechanisms can promote functional reentry. Stabilization of $\mathrm{Ca}^{2+}$ handling may therefore not only reduce the incidence of arrhythmogenic triggers but also prevent their reentrant perpetuation.

Although current pharmacological interventions do not allow specific targeting of SCR or measurement of cellular $\mathrm{Ca}^{2+}$ handling in the in-vivo dog heart, we found that $\mathrm{I}_{\mathrm{CaL}}$ inhibition with verapamil could prevent aftercontractions and TdP in our model of LQT1, and this was associated with a reduction in the paradoxical increase in QT and $\mathrm{T}_{\text {peak }}-\mathrm{T}_{\text {end }}$ intervals following ISO. 
We have also shown that the combination of ISO and fast pacing can give rise to diastolic SCR which prolongs APD sufficiently such that the next SCR occurs before the end of repolarization, generating an EAD. The common dependence of DADs and EADs on SCR in the presence of ISO has previously been described ${ }^{5,19}$ and is in agreement with recent findings in rabbit ventricular myocytes ${ }^{20}$. Our results provide novel mechanistic insights on the coupling between SCR, APD prolongation and EAD occurrence and illustrate that diastolic SCR is a central element in both triggered activity and repolarization instability. However, the ionic mechanisms of EAD generation, particularly the relative roles of $\mathrm{I}_{\mathrm{NCX}}$ and $\mathrm{I}_{\mathrm{CaL}}$ are complex and cannot be fully determined based on our data. Moreover, the measurement of the lag between the start of the aftercontraction and the EAD upstroke does not take into account the delay between SCR and activation of contraction or the SCR-induced slowing of repolarization in the priming phase before the EAD upstroke. This lag is likely due to differential $\mathrm{Ca}^{2+}$ thresholds for the activation of contraction versus the activation of membrane currents.

APD prolongation after SCR results from increased $\mathrm{I}_{\mathrm{CaL}}$, which enhances $\mathrm{Ca}^{2+}$ loading via increased sarcolemmal $\mathrm{Ca}^{2+}$ influx. Enhanced loading along with reduced $\mathrm{Ca}^{2+}$ efflux serves to restore and fine-tune SR $\mathrm{Ca}^{2+}$ content to maintain CICR efficacy, as has previously been described in rat ventricular myocytes ${ }^{32,34}$. However, during increased $\mathrm{Ca}^{2+}$ load these mechanisms will readjust, promoting SCR, facilitating the occurrence of afterdepolarizations. Consistent with this reasoning, we found that the probability of observing a DAD was not significantly altered by the presence of a DAD on the previous beat. This strongly implies that, under our experimental conditions, the reduction in myocyte $\mathrm{Ca}^{2+}$ load during a DAD is overcome by the increased sarcolemmal $\mathrm{Ca}^{2+}$ influx during the following prolonged AP. Thus, APD prolongation after SCR contributes to the vicious cycle of $\mathrm{Ca}^{2+}$ loading and ultimately overload. These results are in agreement with the recent modeling study by Morotti et al. who established that $\mathrm{I}_{\mathrm{CaL}}$ predominantly inactivates due to $\mathrm{CDI}$ and that (strongly) reduced CDI can cause $\mathrm{Ca}^{2+}$ overload and DADs ${ }^{40}$.

Inhibition of SCR by ryanodine and tetracaine has previously been described ${ }^{19,} 22$, and the results presented here agree with those data. We extend these observations by showing a concomitant decrease in BVR. Both ryanodine and tetracaine are useful for mechanistic studies but due to their deleterious effects in vivo cannot be used as therapeutic agents. In contrast, both magnesium and flecainide are commonly used antiarrhythmic agents. Here we show that both these agents can lead to a reduction in BVR and arrhythmogenic events, most likely due to stabilization of $\mathrm{Ca}^{2+}$ handling in the single myocyte. Similarly, we confirm the usefulness of modulating Calmodulin/CaMKII during increased $\mathrm{Ca}^{2+}$ loading as an antiarrhythmic strategy ${ }^{24}$. CaMKII phosphorylation has been shown to induce a different gating mode (mode 2 ) of the $\mathrm{I}_{\mathrm{CaL}}$ channel, thereby reducing inactivation, favoring $\mathrm{Ca}^{2+}$ entry and 
the occurrence of EADs and DADs ${ }^{41}$. Interestingly, when CAMKII inhibition is applied a disconnect appears between SCR events and APD. Whether this is due to alterations in $\mathrm{Ca}^{2+}$-dependent inactivation, or due to effects on other CAMKII substrates, such as $\mathrm{I}_{\mathrm{NaL}}$, is not clear and further investigation is warranted. In this regard, the development of selective CaMKII modulators suitable for antiarrhythmic interventions in humans is awaited.

\subsubsection{Conclusions}

We have shown that after SCR, inactivating $\mathrm{I}_{\mathrm{CaL}}$ is increased and APD and QT intervals are prolonged, most likely due to reduced CICR-dependent inactivation of this current. The degree of APD prolongation is exacerbated by inhibition of $I_{k s}$. This contributes to increased BVR and spatial dispersion of repolarization during ISO-induced diastolic $\mathrm{Ca}^{2+}$ release and, aside from DAD-mediated triggered activity, may be an additional mechanism contributing to arrhythmogenesis. Pharmacological interventions that regularize SCR or inhibit SCR with or without preserved systolic contractions reduce BVR. Our data provide novel insights into arrhythmogenic mechanisms during increased $\mathrm{Ca}^{2+}$ loading.

\subsection{Acknowledgements}

The authors thank Drs. Chris Pollard and Jean-Pierre Valentin, Department of Safety Pharmacology, Safety Assessment UK, AstraZeneca R\&D, Alderley Park, UK, for active collaboration and for providing cardiac myocytes. Ongoing collaborations with Dr. Yoram Rudy, Washington University in St. Louis, MO, USA and use of his computational resources are gratefully acknowledged.

\subsection{Sources of Funding}

P.G.A.V. is supported by a Vidi grant from the Netherlands Organization for Scientific Research (ZonMw 91710365). D.A.E. and A.W.T. are supported by the British Heart Foundation. D.M.J. was financially supported by AstraZeneca Ltd, UK. 


\section{References}

(1) Bers DM. Cardiac excitation-contraction coupling. Nature. 2002;415:198-205.

(2) Stern MD, Capogrossi MC, Lakatta EG. Spontaneous calcium release from the sarcoplasmic reticulum in myocardial cells: mechanisms and consequences. Cell Calcium. 1988;9:247-256.

(3) Zygmunt AC, Goodrow RJ, Weigel CM. $\mathrm{I}_{\mathrm{NaCa}}$ and $\mathrm{I}_{\mathrm{Cl}(\mathrm{Ca})}$ contribute to isoproterenol-induced delayed afterdepolarizations in midmyocardial cells. Am J Physiol. 1998;275:H1979-H1992.

(4) Bers DM. Calcium cycling and signaling in cardiac myocytes. Annu Rev Physiol. 2008;70:23-49.

(5) Volders PGA, Kulcsár A, Vos MA, Sipido KR, Wellens HJ, Lazzara R, Szabo B. Similarities between early and delayed afterdepolarizations induced by isoproterenol in canine ventricular myocytes. Cardiovasc Res. 1997;34:348-359.

(6) Ter Keurs HE, Boyden PA. Calcium and arrhythmogenesis. Physiol Rev. 2007;87:457-506.

(7) Zaniboni M, Pollard AE, Yang L, Spitzer KW. Beat-to-beat repolarization variability in ventricular myocytes and its suppression by electrical coupling. Am J Physiol Heart Circ Physiol. 2000;278:H677-687.

(8) Hinterseer M, Beckmann BM, Thomsen MB, Pfeufer A, Ulbrich M, Sinner MF, Perz S, Wichmann HE, Lengyel C, Schimpf R, Maier SK, Várro A, Vos MA, Steinbeck G, Kääb S. Usefulness of short-term variability of QT intervals as a predictor for electrical remodeling and proarrhythmia in patients with nonischemic heart failure. Am J Cardiol. 2010;106:216-220.

(9) Tereshchenko LG, Han L, Cheng A, Marine JE, Spragg DD, Sinha S, Dalal D, Calkins $\mathrm{H}$, Tomaselli GF, Berger RD. Beat-to-beat three-dimensional ECG variability predicts ventricular arrhythmia in ICD recipients. Heart Rhythm. 2010;7:1606-1613.

(10) Thomsen MB, Verduyn SC, Stengl M, Beekman JD, de Pater G, van Opstal J, Volders PGA, Vos MA. Increased short-term variability of repolarization predicts d-sotalol-induced torsades de pointes in dogs. Circulation. 2004; 110:2453-2459. 
(11) Gallacher DJ, Van de Water A, van der Linde H, Hermans AN, Lu HR, Towart $\mathrm{R}$, Volders PGA. In vivo mechanisms precipitating torsades de pointes in a canine model of drug-induced long-QT1 syndrome. Cardiovasc Res. 2007;76:247-256.

(12) Jacobson I, Carlsson L, Duker G. Beat-by-beat QT interval variability, but not QT prolongation per se, predicts drug-induced torsades de pointes in the anaesthetised methoxamine-sensitized rabbit. J Pharmacol Toxicol Methods. $2011 ; 63: 40-46$.

(13) Hinterseer M, Beckmann BM, Thomsen MB, Pfeufer A, Dalla Pozza R, Loeff M, Netz $H$, Steinbeck G, Vos MA, Kääb S. Relation of increased short-term variability of QT interval to congenital long-QT syndrome. Am J Cardiol. 2009;103:1244-1248.

(14) Johnson DM, Heijman J, Pollard CE, Valentin JP, Crijns HJ, Abi-Gerges N, Volders PGA. $\mathrm{I}_{\mathrm{Ks}}$ restricts excessive beat-to-beat variability of repolarization during beta-adrenergic receptor stimulation. $J \mathrm{Mol}$ Cell Cardiol. 2010;48:122-130.

(15) Volders PGA, Sipido KR, Vos MA, Spätjens RLHMG, Leunissen JDM, Carmeliet $\mathrm{E}$, Wellens $\mathrm{HJJ}$. Downregulation of delayed rectifier $\mathrm{K}^{+}$currents in dogs with chronic complete atrioventricular block and acquired torsades de pointes. Circulation. 1999;100:2455-2461.

(16) Dibb KM, Rueckschloss U, Eisner DA, Isenberg G, Trafford AW. Mechanisms underlying enhanced cardiac excitation contraction coupling observed in the senescent sheep myocardium. J Mol Cell Cardiol. 2004;37:1171-1181.

(17) Heijman J, Volders PGA, Westra RL, Rudy Y. Local control of $\beta$-adrenergic stimulation: Effects on ventricular myocyte electrophysiology and $\mathrm{Ca}^{2+}$ transient. J Mol Cell Cardiol. 2011;50:863-871.

(18) Katra RP, Laurita KR. Cellular mechanism of calcium-mediated triggered activity in the heart. Circ Res. 2005;96:535-542.

(19) Priori SG, Corr PB. Mechanisms underlying early and delayed afterdepolarizations induced by catecholamines. $\mathrm{Am} J$ Physiol. 1990;258:H1796-H1805. 
(20) Zhao Z, Wen H, Fefelova N, Allen C, Baba A, Matsuda T, Xie LH. Revisiting the Ionic Mechanisms of Early Afterdepolarizations in Cardiomyocytes: Predominant by Ca Waves or Ca Currents? Am J Physiol Heart Circ Physiol. 2012; In press.

(21) Overend CL, Eisner DA, O'Neill SC. The effect of tetracaine on spontaneous $\mathrm{Ca}^{2+}$ release and sarcoplasmic reticulum calcium content in rat ventricular myocytes. J Physiol. 1997;502 ( Pt 3):471-479.

(22) Venetucci LA, Trafford AW, Diaz ME, O'Neill SC, Eisner DA. Reducing ryanodine receptor open probability as a means to abolish spontaneous $\mathrm{Ca}^{2+}$ release and increase $\mathrm{Ca}^{2+}$ transient amplitude in adult ventricular myocytes. Circ Res. 2006;98:1299-1305.

(23) Trafford AW, Sibbring GC, Diaz ME, Eisner DA. The effects of low concentrations of caffeine on spontaneous $\mathrm{Ca}$ release in isolated rat ventricular myocytes. Cell Calcium. 2000;28:269-276.

(24) Anderson ME. Multiple downstream proarrhythmic targets for calmodulin kinase II: moving beyond an ion channel-centric focus. Cardiovasc Res. 2007;73:657-666.

(25) Curran J, Hinton MJ, Ríos E, Bers DM, Shannon TR. $\beta$-Adrenergic enhancement of sarcoplasmic reticulum calcium leak in cardiac myocytes is mediated by calcium/calmodulin-dependent protein kinase. Circ Res. 2007; 100:391-398.

(26) Kaseda S, Gilmour RF, Jr., Zipes DP. Depressant effect of magnesium on early afterdepolarizations and triggered activity induced by cesium, quinidine, and 4-aminopyridine in canine cardiac Purkinje fibers. Am Heart $J$. $1989 ; 118: 458-466$.

(27) Aomine M, Tatsukawa Y, Yamato T, Yamasaki S. Antiarrhythmic effects of magnesium on rat papillary muscle and guinea pig ventricular myocytes. Gen Pharmacol. 1999;32:107-114.

(28) Watanabe H, Chopra N, Laver D, Hwang HS, Davies SS, Roach DE, Duff HJ, Roden DM, Wilde AA, Knollmann BC. Flecainide prevents catecholaminergic polymorphic ventricular tachycardia in mice and humans. Nat Med. 2009;15:380-383. 
(29) Trafford AW, Diaz ME, Eisner DA. Ca-activated chloride current and $\mathrm{Na}-\mathrm{Ca}$ exchange have different timecourses during sarcoplasmic reticulum Ca release in ferret ventricular myocytes. Pflugers Arch. 1998;435:743-745.

(30) Alseikhan BA, DeMaria CD, Colecraft HM, Yue DT. Engineered calmodulins reveal the unexpected eminence of $\mathrm{Ca}^{2+}$ channel inactivation in controlling heart excitation. Proc Natl Acad Sci U S A. 2002;99:17185-17190.

(31) Myles RC, Wang L, Kang C, Bers DM, Ripplinger CM. Local $\beta$-Adrenergic Stimulation Overcomes Source-Sink Mismatch to Generate Focal Arrhythmia. Circ Res. 2012;110:1454-1464.

(32) Trafford AW, Diaz $\mathrm{ME}$, Negretti N, Eisner DA. Enhanced $\mathrm{Ca}^{2+}$ current and decreased $\mathrm{Ca}^{2+}$ efflux restore sarcoplasmic reticulum $\mathrm{Ca}^{2+}$ content after depletion. Circ Res. 1997;81:477-484.

(33) Papp Z, Peineau N, Szigeti G, Argibay J, Kovacs L. Calcium-dependent modulation of the plateau phase of action potential in isolated ventricular cells of rabbit heart. Acta Physiol Scand. 1999;167:119-129.

(34) Takamatsu H, Nagao T, Ichijo $\mathrm{H}$, Adachi-Akahane S. L-type $\mathrm{Ca}^{2+}$ channels serve as a sensor of the SR $\mathrm{Ca}^{2+}$ for tuning the efficacy of $\mathrm{Ca}^{2+}$-induced $\mathrm{Ca}^{2+}$ release in rat ventricular myocytes. J Physiol. 2003;552:415-424.

(35) Spencer $\mathrm{CI}$, Sham JS. Effects of $\mathrm{Na}^{+} / \mathrm{Ca}^{2+}$ exchange induced by $\mathrm{SR} \mathrm{Ca}^{2+}$ release on action potentials and afterdepolarizations in guinea pig ventricular myocytes. Am J Physiol Heart Circ Physiol. 2003;285:H2552-2562.

(36) Burashnikov A, Antzelevitch C. Block of $\mathrm{I}_{\mathrm{Ks}}$ does not induce early afterdepolarization activity but promotes $\beta$-adrenergic agonist-induced delayed afterdepolarization activity. J Cardiovasc Electrophysiol. 2000;11:458-465.

(37) Lerche C, Seebohm G, Wagner CI, Scherer CR, Dehmelt L, Abitbol I, Gerlach $U$, Brendel J, Attali B, Busch AE. Molecular impact of MinK on the enantiospecific block of $\mathrm{I}_{\mathrm{Ks}}$ by chromanols. $\mathrm{Br} J$ Pharmacol. 2000;131:1503-1506.

(38) Barandi L, Virag L, Jost N, Horvath Z, Koncz I, Papp R, Harmati G, Horvath B, Szentandrassy N, Banyasz T, Magyar J, Zaza A, Varro A, Nanasi PP. Reverse rate-dependent changes are determined by baseline action potential duration in mammalian and human ventricular preparations. Basic Res Cardiol. 2010;105:315-323. 
(39) Sipido KR, Volders PGA, de Groot SHM, Verdonck F, Van de Werf F, Wellens $\mathrm{HJJ}$, Vos MA. Enhanced $\mathrm{Ca}^{2+}$ release and $\mathrm{Na} / \mathrm{Ca}$ exchange activity in hypertrophied canine ventricular myocytes: potential link between contractile adaptation and arrhythmogenesis. Circulation. 2000;102:2137-2144.

(40) Morotti S, Grandi E, Summa A, Ginsburg KS, Bers DM. Theoretical study of Ltype $\mathrm{Ca}^{2+}$ current inactivation kinetics during action potential repolarization and early afterdepolarizations. J Physiol. 2012;590:4465-4481.

(41) Hashambhoy YL, Winslow RL, Greenstein JL. CaMKII-induced shift in modal gating explains L-type $\mathrm{Ca}^{2+}$ current facilitation: a modeling study. Biophys J. 2009;96:1770-1785. 


\section{Supplementary Methods}

\section{Cell-I solation Procedure}

Adult female beagle dogs were used for the myocyte isolations. Anesthesia was induced with $45 \mathrm{mg} / \mathrm{kg}$ pentobarbital. Once full anesthesia was reached, the chest was opened via a left thoracotomy and the heart was excised and placed in an $\mathrm{O}_{2}$-gassed $\mathrm{Ca}^{2+}$-free standard buffer solution at approximately $4^{\circ} \mathrm{C}$. The cell-isolation procedure was the same as previously described ${ }^{1}$. Briefly the left anterior descending coronary artery was cannulated and perfused. After $\sim 20 \mathrm{~min}$ of collagenase perfusion and subsequent washout of the enzyme, the epicardial surface layer was removed from the LV wedge until a depth of $\geq 3 \mathrm{~mm}$ was reached. Softened tissue samples were collected from the midmyocardial layer underneath while contamination with the endocardium was avoided. Samples were gently agitated, filtered and washed. LV midmyocytes were stored at room temperature in standard buffer solution (vide infra) and only quiescent rod-shaped cells with clear cross-striations were used for the experiments. Cells were used within $48 \mathrm{~h}$ of isolation.

\section{Sharp-Electrode Action Potential Recordings}

Transmembrane action potentials (APs) were recorded at $37^{\circ} \mathrm{C}$ using high-resistance (30-60 M $\Omega$ ) glass microelectrodes filled with $3 \mathrm{~mol} / \mathrm{L} \mathrm{KCl}$ with a microelectrode amplifier (Axoclamp-2B, Axon Instruments, Inc). Intracellular pacing was done at various cycle lengths (CLs; $500 \mathrm{~ms}-2000 \mathrm{~ms}$ ). Only cells showing a stable spikeand-dome AP morphology and resting membrane potential were accepted for the experiments. Myocyte contractions were recorded with a video edge motion detector (Crescent Electronics, Sandy, UT, USA).

\section{$\mathrm{Ca}^{2+}$ Measurements}

Isolated myocytes were loaded with the acetoxymethyl ester of indicator Fluo-3 or Fura-2 (Molecular Probes; $5 \mu \mathrm{mol} / \mathrm{L}, 5 \mathrm{~min}$ loading and $30 \mathrm{~min}$ de-esterification). Electrophysiological control for current clamp and voltage clamp experiments with simultaneous $\left[\mathrm{Ca}^{2+}\right]_{\mathrm{i}}$ measurements was achieved using the perforated patch clamp technique with amphotericin-B $(240 \mu \mathrm{g} / \mathrm{ml})$. The switch-clamp facility (frequency $1-3$ $\mathrm{kHz}$ and gain $1-3$ ) of the Axoclamp-2B voltage clamp amplifier (Axon Instruments, CA, USA) was used to overcome the access resistance of the perforated patch. All experiments were performed at $37^{\circ} \mathrm{C}$. 


\section{Drugs and Experimental Solutions}

The standard buffer solution used for the experiments was composed of (in mmol/L): $\mathrm{NaCl} 145, \mathrm{KCl} 4.0, \mathrm{CaCl}_{2} 1.8, \mathrm{MgCl}_{2}$ 1.0, glucose 11 and HEPES 10, pH 7.4 with $\mathrm{NaOH}$ at $37^{\circ} \mathrm{C}$. In a subset of experiments, extracellular $\left[\mathrm{Mg}^{2+}\right]$ was increased to $5 \mathrm{mmol} / \mathrm{L}$ by the addition of $4 \mathrm{mmol} / \mathrm{L} \mathrm{MgSO}_{4}$. In other experiments, extracellular $\left[\mathrm{Ca}^{2+}\right]$ was increased to $3.6 \mathrm{mmol} / \mathrm{L}$ to further increase cellular $\mathrm{Ca}^{2+}$ loading.

$500 \quad \mathrm{nmol} / \mathrm{L} \quad \mathrm{HMR} 1556 \quad((3 \mathrm{R}, 4 \mathrm{~S})-(+)-\mathrm{N}-[3-$ hydroxy-2,2-dimethyl-6-(4,4,4trifluorobutoxy)chroman-4-yl]-N-methylmethanesulfonamide; a gift from Dr. $\mathrm{H}$. Gögelein, Sanofi-Aventis Germany $\mathrm{GmbH}$, Frankfurt, Germany) was used to selectively and completely block $\mathrm{I}_{\mathrm{Ks}}{ }^{2}{ }^{4}$. Sarcoplasmic reticulum (SR) $\mathrm{Ca}^{2+}$ release through the ryanodine receptor (RyR) was inhibited with $1 \mu \mathrm{mol} / \mathrm{L}$ ryanodine (RBI, Natick, MA, USA) or stabilized using $5 \mu \mathrm{mol} / \mathrm{L}$ tetracaine (Sigma, Zwijndrecht, The Netherlands). Caffeine (Sigma, Zwijndrecht, The Netherlands; maximally $500 \mu \mathrm{mol} / \mathrm{L}$ ) was used to increase RyR open probability. $\mathrm{Ca}^{2+} /$ Calmodulin-dependent kinase (CaMKII) activity was modulated using the CaMKII inhibitor KN93 (Sigma, Zwijndrecht, The Netherlands; $5 \mu \mathrm{mol} / \mathrm{L}$ ) and the calmodulin inhibitor W7 (Tocris Bioscience, Bristol, UK; $1 \mu \mathrm{mol} / \mathrm{L})$. The class-1C antiarrhythmic agent flecainide (MedaPharma, Amstelveen, The Netherlands) was employed in a subset of experiments. At $6 \mu \mathrm{mol} / \mathrm{L}$, flecainide results in a $40 \%$ decrease in RyR activity ${ }^{5}$ as well as affecting multiple other targets (40\% inhibition of $\mathrm{I}_{\mathrm{TO}}{ }^{6}$, significant inhibition of (late) $\mathrm{I}_{\mathrm{Na}}{ }^{7}$ and $\sim 50 \%$ inhibition of HERG $\mathrm{K}^{+}$channels ${ }^{8}$ ).

For perforated patch experiments the patch pipette solution contained (in $\mathrm{mmol} / \mathrm{L}$ ): $\mathrm{KCH}_{3} \mathrm{O}_{3} \mathrm{~S} 125, \mathrm{KCl} 20, \mathrm{NaCl} 10$, HEPES 10, $\mathrm{MgCl}_{2} 5, \mathrm{~K}_{2}$ EGTA 0.1 , titrated to 7.2 with $\mathrm{KOH}$ with patch pipette resistance being 2-3 $\mathrm{M} \Omega$. In voltage clamp experiments, $\mathrm{K}^{+}$currents were blocked by addition of $500 \mathrm{nmol} / \mathrm{L}$ HMR1556, $5 \mathrm{mmol} / \mathrm{L}$ 4-aminopyridine and $100 \mathrm{nmol} / \mathrm{L} \mathrm{BaCl}_{2}$ to the standard buffer solution. Extracellular $\mathrm{Ca}^{2+}$ was raised (to 3.6 or $5.0 \mathrm{mmol} / \mathrm{L}$ ) to further enhance cellular $\mathrm{Ca}^{2+}$ loading.

HMR1556, ryanodine, tetracaine, caffeine, W7 and KN93 were initially dissolved in dimethyl sulfoxide (DMSO) and then diluted so that the concentration of the solvent was maximally $0.1 \%$ in the superfusate, a concentration that has no measurable effects on AP or ionic currents.

BAR stimulation was applied by $100 \mathrm{nmol} / \mathrm{L}$ isoproterenol (ISO) in all experiments. This agent was originally dissolved in distilled water containing $30 \mu \mathrm{mol} / \mathrm{L}$ ascorbic acid and then stored in the dark at $4^{\circ} \mathrm{C}$ until use.

When assessing the effect of pharmacological interventions, all conditions were kept constant for several minutes until APD and cell shortening reached steady state. Analyses were performed using these steady-state data. 


\section{Data Analysis}

Detection of EADs, DADs and determination of APD, BVR and DAD parameters was done in a semi-automated fashion using a custom Matlab (The Mathworks, Natick, MA, USA) script. Briefly, Axon (Axon Instruments, CA, USA) data files were loaded into Matlab. AP upstroke was detected based on peaks in the numerically calculated $\mathrm{dV} / \mathrm{dt}$, and $A \mathrm{PD}_{90}$ was determined. Subsequently, the diastolic interval between $A P D_{90}$ of one beat and the point of maximum $\mathrm{dV} / \mathrm{dt}$ of the next beat was processed for all beats. The signal was low-pass filtered at $30 \mathrm{~Hz}$ to reduce noise. All peaks in the filtered signal were determined and resting membrane potential was defined as the average value between the smallest (most negative) local maximum and local minimum. The amplitude of all other peaks was determined relative to this resting membrane potential. DADs were identified as a local maximum in the filtered signal of more than $2 \mathrm{mV}$ in amplitude and manually confirmed by the investigators. EADs were detected as positive local maxima in the low-pass filtered $\mathrm{dV} / \mathrm{dt}$ signal preceded and followed by negative local minima. Detection of EADs was manually validated.

BVR was determined using a sliding window of consecutive 30 beats using: $\Sigma\left(\mid A P D_{i+1}\right.$ - $\left.A P D_{i} \mid\right) /\left[n_{\text {beats }} \times \sqrt{ } 2\right]$, and mean BVR for a given condition (CL and/or pharmacological intervention) was determined for each cell.

\section{In-Vivo Dog Model of LQT1}

TdP arrhythmias were induced in an in-vivo dog model of long QT 1 syndrome as previously described ${ }^{9}$. Briefly, general anesthesia was induced in 10 beagle dogs by lofentanil (0.075 mg/kg body weight i.v.), scopolamine $(0.015 \mathrm{mg} / \mathrm{kg})$, succinylcholine $(1.0 \mathrm{mg} / \mathrm{kg})$, hourly slow injections of fentanyl $(0.025 \mathrm{mg} / \mathrm{kg}$ i.v.), and continuous infusion of etomidate $(1.5 \mathrm{mg} / \mathrm{kg} / \mathrm{hour})$. Dogs were ventilated with $30 \%$ oxygen in pressurized air to normocapnia. The body temperature was kept at $37^{\circ} \mathrm{C}$ with a heated water mattress. ECG standard lead II was continuously recorded and the QT interval (QT; ms) measured from the onset of the QRS to the final end of the $T$ wave. Left ventricular (LV) intracavitary pressures were recorded with high-fidelity catheter-tip micromanometers (Gaeltec Ltd, Dunvegan, UK and Millar Instruments Inc, Houston, TX). Under fluoroscopic guidance a MAP catheter (Boston Scientific-EP Technologies, San Jose, CA, USA) was placed at the endocardium of the LV and RV, near the apical septum. HMR1556 (dissolved in 20\% HP- $\beta$-cyclodextrin) was infused i.v. in the dogs, initially at a rate of $0.025 \mathrm{mg} / \mathrm{kg} / \mathrm{min}$ for $30 \mathrm{~min}$ and followed (if necessary) by infusions at $0.05 \mathrm{mg} / \mathrm{kg} / \mathrm{min}$ and $0.1 \mathrm{mg} / \mathrm{kg} / \mathrm{min}$. At regular time intervals, boluses of ISO $(1.25,2.5$ or $5 \mu \mathrm{g} / \mathrm{kg})$ were injected to induce TdP and in prevention experiments verapamil $(0.4 \mathrm{mg} / \mathrm{kg})$ was infused pre-ISO challenge after confirming TdP inducibility with HMR1556 + ISO in the same animal. External electrical cardioversion was applied to terminate sustained TdP or its deterioration into ventricular fibrillation, if induced by ISO. 


\section{Computational Modelling}

A recent model of the canine ventricular myocyte electrophysiology including $\beta A R$ stimulation ${ }^{10}$ was extended to induce diastolic $\mathrm{SR} \mathrm{Ca}^{2+}$ release in a controlled fashion, similar to a recent approach by Xie et al. ${ }^{11}$. Both timing (start, duration) and amplitude of diastolic SR $\mathrm{Ca}^{2+}$ release could be controlled.

In particular, the model was divided into two identical domains (Figure 1) coupled by $\mathrm{Ca}^{2+}$ diffusion between cytosol and network SR to simulate the local origin of SCR. Diffusion time constants were based on the local control model by Restrepo et al. ${ }^{12}$. and diffusion was significantly slower in SR compared to cytosol, consistent with experimental observations ${ }^{13}$. The late component of $\mathrm{I}_{\mathrm{Na}}\left(\mathrm{I}_{\mathrm{NaL}}\right)$ was increased in the model to simulate the midmyocardial origin of the myocytes used in our experiments, consistent with experimental data from Zygmunt et al. ${ }^{14}$. Model APD rate dependence at baseline, in the presence of ISO and in the presence of ISO+HMR1556 (simulated as $100 \%$ inhibition of $\mathrm{I}_{\mathrm{Ks}}$ ) was consistent with experimental data (Figure 2A). Parameters of the L-type $\mathrm{Ca}^{2+}$ current were adjusted based on the experiments in the presence of ryanodine to obtain quantitative agreement on the amount of APD prolongation in the absence of SR $\mathrm{Ca}^{2+}$ release.

To initiate SCR, steady-state RyR activation was set to a constant value in one of the two domains for the interval of diastolic $\mathrm{SR} \mathrm{Ca}^{2+}$ release such that the desired reduction in local JSR $\mathrm{Ca}^{2+}$ was achieved. When steady-state RyR activation was set to $0.375\left(\mathrm{SCR}_{\text {level }}=0.375\right)$ from $\mathrm{t}=925 \mathrm{~ms}$ until $\mathrm{t}=950 \mathrm{~ms}$ (at $\mathrm{CL}=1000 \mathrm{~ms}$ ), the SCR-induced prolongation of APD was consistent with experimental observations (Figure 2B).

The model was paced to steady state (2000 seconds of pacing) in the presence of $\beta A R$ stimulation with or without $I_{k s}$ inhibition, to mimic experimental conditions. The effect of diastolic SR $\mathrm{Ca}^{2+}$ release was examined by determining APD of a single beat at steady-state as a function of the timing and amplitude of the preceding diastolic $\mathrm{Ca}^{2+}$ release. In some simulations other currents / fluxes ( $\left.\mathrm{I}_{\mathrm{CaL}}, \mathrm{I}_{\mathrm{NaCa}}, \mathrm{I}_{\mathrm{Cl}(\mathrm{Ca})}, \mathrm{J}_{\mathrm{rel}}\right)$ were blocked for the duration of this final beat.

One-dimensional strand simulations were performed as previously described ${ }^{15}$. Steady-state conditions of single-cell simulation in the presence of ISO and $\mathrm{I}_{\mathrm{Ks}}$ blockade were used as initial conditions for the homogeneous strand of 128 cells. The strand was subsequently paced by direct stimulation of the first 3 cells ( -80 $\mathrm{pA} / \mathrm{pF}$ for $2.0 \mathrm{~ms}$ ) for 100 seconds to achieve steady-state conditions for the strand. The effect of diastolic SR $\mathrm{Ca}^{2+}$ release was determined by applying the methodology described above to $k$ cells in the middle of the strand. SR Ca ${ }^{2+}$ release was initiated simultaneously in all $k$ cells. The exact mechanisms by which SCR synchronizes across a sufficient number of myocytes to generate ventricular ectopic beats remain 
incompletely understood. Recent research by Wasserstrom et al. has suggested that an intrinsic synchronization occurs due to a decrease in variability of SCR events with increasing $\mathrm{Ca}^{2+}$ load ${ }^{16,17}$. Furthermore, Myles et al. have shown that localized $\beta A R$ can produce spatiotemporal synchronization of $\mathrm{SR} \mathrm{Ca}^{2+}$ overload and release, which can produce focal activity and arrhythmia in normal rabbit hearts ${ }^{18}$. Although direct diffusion of $\mathrm{Ca}^{2+}$ between cells through gap junctions could further contribute to the synchronization of SCR ${ }^{19}$, this was not implemented in the current model because the main goal of the present research was to determine the electrophysiological consequences of SCR, independent of the synchronization method.

A similar approach was used to study the effect of diastolic SR $\mathrm{Ca}^{2+}$ release on homogeneous two-dimensional sheets of virtual 'tissue'. The tissue size was $2 \times 2 \mathrm{~cm}$ and was simulated using $200 \times 200$ grid points, in line with previous studies ${ }^{20,}{ }^{21}$. Steady-state conditions of single-cell simulations in the presence of ISO and $\mathrm{I}_{\mathrm{Ks}}$ blockade were used as initial conditions and 5 beats were simulated following stimulation of the left-most column of 200 cells $(-80 \mathrm{pA} / \mathrm{pF}$ for $2.0 \mathrm{~ms})$, resulting in a planar wave activating the entire sheet. Conduction velocity was $49 \mathrm{~cm} / \mathrm{s}$. Diastolic SR $\mathrm{Ca}^{2+}$ release was initiated simultaneously in $25 \%$ of the 40.000 cells surrounding the center of the tissues (i.e., cells with $x$ and $y$ coordinates between 50 and 150).

\section{Alterations in Model Equations Compared to Heijman et al. ${ }^{10}$.}

The superscript symbol $x$, is used to designate one of the two identical $\mathrm{Ca}^{2+}$ domains. Whole-cell concentrations / currents are defined as the average of both domains and are indicated without superscript $\mathrm{x}$.

Altered $I_{\text {NaL: }}$

$$
\begin{aligned}
& \mathrm{I}_{\mathrm{NaL}}^{\mathrm{NP}}=1.6 \cdot 6.500 \cdot 10^{-3} \cdot\left(\mathrm{m}_{\mathrm{L}}\right)^{3} \cdot \mathrm{h}_{\mathrm{L}} \cdot\left(\mathrm{V}_{\mathrm{m}}-\mathrm{E}_{\mathrm{Na}}\right) \\
& \mathrm{I}_{\mathrm{NaL}}^{\mathrm{P}, \mathrm{CaMK}}=1.6 \cdot 1.600 \cdot 10^{-2} \cdot\left(\mathrm{m}_{\mathrm{L}}\right)^{3} \cdot \mathrm{h}_{\mathrm{L}} \cdot\left(\mathrm{V}_{\mathrm{m}}-\mathrm{E}_{\mathrm{Na}}\right)
\end{aligned}
$$

Altered $I_{\text {CaL: }}$

$$
\begin{aligned}
& \mathrm{I}_{\mathrm{V}, \mathrm{T}}^{\mathrm{P}}=\frac{1}{\frac{1}{70.0 \cdot\left(1+\exp \left(\left(\mathrm{V}_{\mathrm{m}}+49.10\right) / 10.349\right)\right)}+\frac{1}{75 \cdot\left(1+\exp \left(-\left(\mathrm{V}_{\mathrm{m}}+0.213\right) / 10.807\right)\right)}} \\
& \mathrm{I}_{\mathrm{V}, \infty}^{\mathrm{P}}=\frac{1}{1.02} \cdot\left(0.02+\frac{1}{1+\exp \left(\left(\mathrm{V}_{\mathrm{m}}+29.979\right) / 3.1775\right)}\right) \\
& \mathrm{Is}_{\mathrm{V}, \infty}^{\mathrm{P}}=\frac{1}{1.0004} \cdot\left(0.0004+\frac{1}{1+\exp \left(\left(\mathrm{V}_{\mathrm{m}}+29.979\right) / 3.1775\right)}\right)
\end{aligned}
$$


Altered RyR:

$\mathrm{h}_{\mathrm{R}}=10$

$\mathrm{I}_{\mathrm{Rel}, \infty}^{\mathrm{NP}}=\frac{\mathrm{a}_{\mathrm{Rel}}}{1+\left(\frac{\mathrm{K}_{\mathrm{Rel}, \infty}}{\left[\mathrm{Ca}^{2+}\right]_{\mathrm{JSR}}^{\mathrm{X}}}\right)^{\mathrm{h}_{\mathrm{R}}}} \cdot \frac{\mathrm{I}_{\mathrm{ICaL}}^{\mathrm{X}}}{1+\exp \left(\frac{\left(\mathrm{I}_{\mathrm{ICaL}}^{\mathrm{X}}+1.5\right)}{0.0001}\right)}-\mathrm{SCR}_{\text {level }}$

$\mathrm{I}_{\mathrm{Rel}, \infty}^{\mathrm{P}}=1.9925 \cdot \frac{\mathrm{a}_{\mathrm{Rel}}}{1+\left(\frac{\mathrm{K}_{\mathrm{Rel}, \infty}}{\left[\mathrm{Ca}^{2+}\right]_{\mathrm{JSR}}^{\mathrm{X}}}\right)^{\mathrm{h}_{\mathrm{R}}}} \cdot \frac{\mathrm{I}_{\text {IIaL }}^{\mathrm{X}}}{1+\exp \left(\frac{\left(\mathrm{I}_{\mathrm{ICaL}}^{\mathrm{X}}+1.5\right)}{0.0001}\right)}-\mathrm{SCR}_{\text {level }}$

\section{Altered $\mathrm{Ca}^{2+}$ diffusion:}

$\mathrm{I}_{\mathrm{tr}}^{\mathrm{X}}=\frac{\left[\mathrm{Ca}^{2+}\right]_{\mathrm{NSR}}^{\mathrm{X}}-\left[\mathrm{Ca}^{2+}\right]_{\mathrm{JSR}}^{\mathrm{X}}}{\mathrm{T}_{\mathrm{tr}}}, \mathrm{T}_{\mathrm{tr}}=100 \mathrm{~ms}$

$\mathrm{I}_{\text {Diff,cyt }}^{\mathrm{X}}=\frac{\left[\mathrm{Ca}^{2+}\right]_{\mathrm{i}}^{\mathrm{y}}-\left[\mathrm{Ca}^{2+}\right]_{\mathrm{i}}^{\mathrm{X}}}{\mathrm{T}_{\text {Diff,cyt }}}, \mathrm{T}_{\text {Diff,cyt }}=1.00 \mathrm{~ms}, \mathrm{y}$ : other $\mathrm{Ca}^{2+}$ domain

$\mathrm{I}_{\text {Diff,nsr }}^{\mathrm{X}}=\frac{\left[\mathrm{Ca}^{2+}\right]_{\mathrm{NSR}}^{\mathrm{y}}-\left[\mathrm{Ca}^{2+}\right]_{\mathrm{NSR}}^{\mathrm{x}}}{\mathrm{T}_{\mathrm{Diff}, \mathrm{nsr}}}, \mathrm{T}_{\text {Diff,nsr }}=25.0 \mathrm{~ms}$, y: other $\mathrm{Ca}^{2+}$ domain

$\frac{\mathrm{d}\left[\mathrm{Ca}^{2+}\right]_{i, t}^{\mathrm{x}}}{\mathrm{dt}}=-\left(\frac{\left(\mathrm{I}_{\mathrm{Ca}, \mathrm{b}}^{\mathrm{X}}+\mathrm{I}_{\mathrm{pCa}}^{\mathrm{X}}-2 \cdot \mathrm{I}_{\mathrm{NaCa}, \mathrm{i}}^{\mathrm{X}}\right) \cdot \mathrm{C}_{\mathrm{sc}} \cdot \mathrm{A}_{\mathrm{cap}}}{\mathrm{z}_{\mathrm{Ca}} \cdot \mathrm{F} \cdot \mathrm{V}_{\text {myo }}}+\mathrm{I}_{\mathrm{up}}^{\mathrm{X}} \cdot \frac{\mathrm{V}_{\mathrm{nsr}}}{\mathrm{V}_{\mathrm{myo}}}-\mathrm{I}_{\text {Diff }}^{\mathrm{X}} \cdot \frac{\mathrm{V}_{\mathrm{SS}, \mathrm{SR}}}{\mathrm{V}_{\mathrm{myo}}}-\mathrm{I}_{\mathrm{Diff}, \mathrm{cyt}}^{\mathrm{X}}\right)$

$\frac{\mathrm{d}\left[\mathrm{Ca}^{2+}\right]_{\mathrm{NSR}}^{\mathrm{x}}}{\mathrm{dt}}=\mathrm{I}_{\mathrm{up}}^{\mathrm{x}}-\mathrm{I}_{\mathrm{tr}}^{\mathrm{x}} \cdot \frac{\mathrm{V}_{\mathrm{JSR}}}{\mathrm{V}_{\mathrm{NSR}}}+\mathrm{I}_{\text {Diff,nsr }}^{\mathrm{X}}$ 


\section{Supplemental References}

(1) Volders PGA, Sipido KR, Carmeliet E, Spätjens RLHMG, Wellens HJ, Vos MA. Repolarizing $\mathrm{K}^{+}$currents $\mathrm{I}_{\mathrm{TO} 1}$ and $\mathrm{I}_{\mathrm{Ks}}$ are larger in right than left canine ventricular midmyocardium. Circulation. 1999;99:206-210.

(2) Volders PGA, Stengl M, van Opstal JM, Gerlach U, Spätjens RL, Beekman JD, Sipido KR, Vos MA. Probing the contribution of $I_{K s}$ to canine ventricular repolarization: key role for $\beta$-adrenergic receptor stimulation. Circulation. 2003; 107:2753-2760.

(3) Gögelein $\mathrm{H}$, Bruggemann A, Gerlach U, Brendel J, Busch AE. Inhibition of $\mathrm{I}_{\mathrm{Ks}}$ channels by HMR 1556. Naunyn Schmiedebergs Arch Pharmacol. 2000;362:480-488.

(4) Thomas GP, Gerlach U, Antzelevitch C. HMR 1556, a potent and selective blocker of slowly activating delayed rectifier potassium current. J Cardiovasc Pharmacol. 2003;41:140-147.

(5) Hwang HS, Hasdemir C, Laver D, Mehra D, Turhan K, Faggioni M, Yin H, Knollmann BC. Inhibition of cardiac $\mathrm{Ca}^{2+}$ release channels (RyR2) determines efficacy of class I antiarrhythmic drugs in catecholaminergic polymorphic ventricular tachycardia. Circ Arrhythm Electrophysiol. 2011;4:128-135.

(6) Rosati B, Pan Z, Lypen S, Wang HS, Cohen I, Dixon JE, McKinnon D. Regulation of KChIP2 potassium channel $\beta$ subunit gene expression underlies the gradient of transient outward current in canine and human ventricle. J Physiol. 2001;533:119-125.

(7) Wang GK, Russell C, Wang SY. State-dependent block of wild-type and inactivation-deficient $\mathrm{Na}^{+}$channels by flecainide. $J$ Gen Physiol. 2003;122:365-374.

(8) Paul AA, Witchel HJ, Hancox JC. Inhibition of the current of heterologously expressed HERG potassium channels by flecainide and comparison with quinidine, propafenone and lignocaine. Br J Pharmacol. 2002;136:717-729.

(9) Gallacher DJ, Van de Water A, van der Linde $\mathrm{H}$, Hermans AN, Lu HR, Towart $\mathrm{R}$, Volders PGA. In vivo mechanisms precipitating torsades de pointes in a canine model of drug-induced long-QT1 syndrome. CardiovasC Res. $2007 ; 76: 247-256$. 
(10) Heijman J, Volders PGA, Westra RL, Rudy Y. Local control of $\beta$-adrenergic stimulation: Effects on ventricular myocyte electrophysiology and $\mathrm{Ca}^{2+}$ transient. J Mol Cell Cardiol. 2011;50:863-871.

(11) Xie Y, Sato D, Garfinkel A, Qu Z, Weiss JN. So little source, so much sink: requirements for afterdepolarizations to propagate in tissue. Biophys $\mathrm{J}$. 2010;99:1408-1415.

(12) Restrepo JG, Weiss JN, Karma A. Calsequestrin-mediated mechanism for cellular calcium transient alternans. Biophys J. 2008;95:3767-3789.

(13) Swietach P, Spitzer KW, Vaughan-Jones RD. Modeling calcium waves in cardiac myocytes: importance of calcium diffusion. Front Biosci. 2010;15:661-680.

(14) Zygmunt AC, Eddlestone GT, Thomas GP, Nesterenko VV, Antzelevitch C. Larger late sodium conductance in $\mathrm{M}$ cells contributes to electrical heterogeneity in canine ventricle. Am J Physiol Heart Circ Physiol. 2001;281:H689-697.

(15) Decker KF, Heijman J, Silva JR, Hund TJ, Rudy Y. Properties and ionic mechanisms of action potential adaptation, restitution, and accommodation in canine epicardium. Am J Physiol Heart Circ Physiol. 2009;296:H1017-1026.

(16) Wasserstrom JA, Shiferaw $Y$, Chen W, Ramakrishna S, Patel H, Kelly JE, O'Toole MJ, Pappas A, Chirayil N, Bassi N, Akintilo L, Wu M, Arora R, Aistrup GL. Variability in timing of spontaneous calcium release in the intact rat heart is determined by the time course of sarcoplasmic reticulum calcium load. Circulation research. 2010;107:1117-1126.

(17) Shiferaw $\mathrm{Y}$, Aistrup GL, Wasserstrom JA. Intracellular $\mathrm{Ca}^{2+}$ Waves, Afterdepolarizations, and Triggered Arrhythmias. Cardiovascular Research. 2012; In press.

(18) Myles RC, Wang L, Kang C, Bers DM, Ripplinger CM. Local B-Adrenergic Stimulation Overcomes Source-Sink Mismatch to Generate Focal Arrhythmia. Circ Res. 2012;110:1454-1464.

(19) Lamont C, Luther PW, Balke CW, Wier WG. Intercellular $\mathrm{Ca}^{2+}$ waves in rat heart muscle. J Physiol. 1998;512:669-676. 
(20) Priori SG, Corr PB. Mechanisms underlying early and delayed afterdepolarizations induced by catecholamines. $\mathrm{Am} \mathrm{J}$ Physiol. 1990;258:H1796-H1805.

(21) Zou R, Kneller J, Leon $\sqcup$, Nattel S. Substrate size as a determinant of fibrillatory activity maintenance in a mathematical model of canine atrium. American journal of physiology. Heart and circulatory physiology. 2005;289:H1002-1012. 


\section{Chapter 5}

\section{Interventricular Differences in $\beta$-Adrenergic Responses in the Canine Heart: Role of Phosphodiesterases}

Manuscript under Revision

CEM and DMJ contributed equally to this work

Cristina E Molina • Daniel M Johnson • Hind Mehel • Roel LHMG Spätjens • Delphine Mika • Vincent Algalarrondo • Zeineb Haj Slimane • Patrick Lechêne • Najah Abi-Gerges • Henk van der Linde • Jerome Leroy • Paul GA Volders • Rodolphe Fischmeister • Grégoire Vandecasteele 


\section{Abstract}

Right (RV) and left ventricles (LV) have different embryologic, structural, metabolic and electrophysiologic characteristics, but whether interventricular differences exist in $\beta$-adrenergic receptor ( $\beta A R$ ) responsiveness is unknown. This study was designed to examine whether the $\beta A R$ differs in RV versus LV and study the associated signaling mechanisms. LV and RV intracavitary pressures were recorded in anesthetized beagle dogs. Sarcomere shortening, $\mathrm{Ca}^{2+}$ transients, $\mathrm{I}_{\mathrm{CaL}}$ and $\mathrm{I}_{\mathrm{Ks}}$ currents were recorded in isolated dog LV and RV midmyocytes. Intracellular [CAMP] and PKA activity were measured by live cell imaging using FRET-based sensors.

A bolus injection of isoproterenol increased $\mathrm{RV} \mathrm{dP} / \mathrm{dt}_{\max } \sim 5$-fold versus 3-fold in $\mathrm{LV}$. Isoproterenol increased sarcomere shortening $\sim 10$-fold and $\mathrm{Ca}^{2+}$-transient amplitude $\sim 2$-fold in LV midmyocytes (LVMs) versus 25-fold and 3-fold in RVMs. FRET imaging using targeted Epac2camps sensors revealed no change in subsarcolemmal [CAMP], but a 2-fold higher $\beta$-AR stimulation of cytoplasmic [CAMP] in RVMs versus LVMs. Accordingly, $\beta A R$ regulation of $\mathrm{I}_{\mathrm{CaL}}$ and $\mathrm{I}_{\mathrm{Ks}}$ were similar between LVMs and RVMs, whereas cytoplasmic PKA activity was increased in RVMs. Both PDE3 and PDE4 contributed to the $\beta A R$ regulation of cytoplasmic [CAMP], and the difference between LVMs and RVMs was abolished by PDE3 inhibition and attenuated by PDE4 inhibition. In conclusion canine RV and LV differ in their $\beta A R$ response due to intrinsic differences in myocyte $\beta$-AR downstream signaling. Enhanced $\beta A R$ responsiveness of the RV results from higher cAMP elevation in the cytoplasm, due to a decreased degradation by PDE3 and PDE4 in the RV compared to the LV. 


\subsection{I ntroduction}

The sympathetic nervous system is responsible for adaptation of cardiac output to stress and physical exercise. This "fight-or-flight" response is mediated primarily by noradrenaline and adrenaline acting on $\beta$-adrenergic receptors ( $\beta A R s)$ at the surface of cardiac myocytes. $\beta$ ARs are coupled through $\mathrm{G}_{\mathrm{as}}$ to adenylyl cyclases (AC) and the generation of CAMP, which in turn activates the CAMP-dependent protein kinase (PKA). PKA then phosphorylates key proteins involved in excitation-contraction coupling (ECC) including sarcolemmal L-type $\mathrm{Ca}^{2+}$ channels $\left(\mathrm{I}_{\mathrm{CaL}}\right)$, ryanodine receptors (RyR2), phospholamban and troponin I ${ }^{1}$. In addition, PKA phosphorylates slowly-activating delayed rectifier $\mathrm{K}^{+}\left(\mathrm{I}_{\mathrm{KS}}\right)$ channels to control cardiac repolarization ${ }^{2}$.

The levels of CAMP and thus the degree of PKA activation are finely regulated by cyclic nucleotide phosphodiesterases (PDEs) that degrade the second messenger into 5'-AMP. Cardiac PDEs degrading CAMP belong to 5 families (PDE1-4 and PDE8) which can be distinguished by distinct enzymatic properties and pharmacology ${ }^{3}$. In rodents, PDE3 and PDE4 are the major contributors to the total CAMP-hydrolytic activity ${ }^{4,5}$ and PDE4 is dominant to modulate $\beta A R$ regulation of cAMP levels ${ }^{6-9}$. Multiple PDE4 variants associate with $\beta$ ARs ${ }^{10-12}$, RyR2 ${ }^{13}$, SERCA2 ${ }^{14,15}, \mathrm{I}_{\mathrm{CaL}}{ }^{16}$ and $\mathrm{I}_{\mathrm{ks}}$ ${ }^{17}$ to exert local control of ECC. In larger mammals, PDE3 activity is dominant in microsomal fractions ${ }^{18-20}$ and PDE3 inhibitors exert a potent positive inotropic effect ${ }^{21}$. Selective inhibition of PDE3 with milrinone has been shown to improve cardiac contractility in patients with congestive heart failure ${ }^{22}$. The role of PDE4 is less well defined but evidence is emerging that PDE4 may also play an important role in these species. In the canine heart, a large PDE4 activity is found in the cytoplasm ${ }^{18}$ but PDE4 is also present in microsomal fractions, where it accounts for $\sim 20 \%$ of the activity ${ }^{19}$. Recent studies have indicated that PDE4 is expressed in human ventricle where, similar to rodents, it associates with BARs, RyR2 and phospholamban ${ }^{5,}{ }^{13}$. Moreover, PDE4 controls ECC and arrhythmias in human atrium ${ }^{23}$.

The right (RV) and left (LV) ventricles originate from different progenitor cells ${ }^{24,} 25$ and differ in several important ways. Both ventricles have different mass (under normal conditions LV mass is 6-fold RV mass), volume, morphologies and pressures 26,27 . Electrical heterogeneity has been well characterized between ventricular epicardial, endocardial and midmyocardial layers ${ }^{28-31}$. Although less studied, different electrophysiological properties were also reported between the two ventricles. In rats and dog, the action potential (AP) is shorter in the RV than in the $\mathrm{LV}^{32}, 33$. In dog, the notch in phase 1 of the AP is deeper in the RV than the LV ${ }^{33}, 34$. These differences are related to larger repolarizing $\mathrm{K}^{+}$currents, $\mathrm{I}_{\mathrm{TO}}$ and $\mathrm{I}_{\mathrm{Ks}}$ in dog RV ${ }^{33}$, 34. In this species, a larger RV $\mathrm{I}_{\mathrm{Ks}}$ correlates with a higher expression of KCNQ1 and KCNE1, the principal and auxiliary subunit of the $\mathrm{I}_{\mathrm{KS}}$ channel, respectively ${ }^{35}$. 
Regional heterogeneity between the LV and the RV may also exist for ATP-activated $\mathrm{K}^{+}$current, $\mathrm{I}_{\text {KATP }} 36,37$.

In contrast to these electrophysiological studies, a recent proteomic study reported no difference in the expression level of more than 600 proteins between the RV and the LV from pig and rabbit. Most of these were contractile/structural proteins, oxidative phosphorylation components, and enzymes from intermediary metabolism. No plasma membrane voltage-gated ion channels were analyzed, but a few major $\mathrm{Ca}^{2+}$ handling proteins (SERCA2, RyR2) and signal transduction components $\left(\mathrm{Ca}^{2+} /\right.$ Calmodulin kinase IIס, PKA type I and II) showed no variation in expression level ${ }^{38}$.

These studies raise the question of whether functional differences between the LV and the RV are limited to electrophysiological features or extend to other aspects of ventricular function. Surprisingly, only limited information is available concerning ECC and its neurohumoral regulation in RV versus LV comparisons. However, previous reports in rat have shown that, despite similar interventricular expression levels, a higher proportion of the total SERCA2a pool is associated with phospholamban, resulting in lower $\mathrm{Ca}^{2+}$ reuptake rate and prolonged $\mathrm{Ca}^{2+}$ transients in $\mathrm{RV}$ myocytes compared with LV myocytes ${ }^{39}$. In mouse, opposite inotropic responses to $\alpha_{1}$-AR stimulation were reported, and attributed to different effects on myofilament $\mathrm{Ca}^{2+}$ sensitivity ${ }^{40}$. In human, recent clinical studies indicate that ventricular load increases more for the RV than the LV during exercise ${ }^{41}$ and this may reflect, at least partly, the relative differences in maximal pulmonary-arterial versus aortic pressure rise. It is currently unclear whether interventricular differences exist in the sympathetic responsiveness of the human heart.

In canine, stimulation of the cardiac sympathetic nerves induces greater changes in contractile force in the RV than in the LV ${ }^{42}$. These changes are insensitive to $\alpha_{1}-A R$ blockade by phentolamine, implicating differences in $\beta A R$ response ${ }^{43}$. However, several studies reported no difference in $\beta A R$ density, AC activity and its activation by catecholamines between RV and LV under normal conditions ${ }^{44-46}$.

Intrigued by these apparent differences, we examined the $\beta A R$ regulation of cardiac contractility in dog RV and LV in vivo and in isolated myocytes. Our data reveal enhanced sensitivity of the RV to $\beta A R$ stimulation, and provide evidence that PDE3 and PDE4 shape distinct compartmentalized CAMP signals that underlie interventricular dispersion in $\beta A R$ stimulation. 


\subsection{Materials and Methods}

This investigation conformed with the Guide for the Care and Use of Laboratory Animals published by the US National Institutes of Health (NIH Publication No. 85-23, revised 1996). Animal handling was in accordance with the European Directive for the Protection of Vertebrate Animals Used for Experimental and Other Scientific Purposes (86/609/EU). The study was conducted in accordance with the Declaration of Helsinki principles, and approved by the Ethical Committees of our institutions.

\subsubsection{In Vivo Experiments}

General anesthesia was induced in 4 beagle dogs $(3 \mathrm{~F} / 1 \mathrm{M}$; average weight $11 \pm 1 \mathrm{~kg})$ by lofentanil $(0.075 \mathrm{mg} / \mathrm{kg}$ body weight i.v.), scopolamine $(0.015 \mathrm{mg} / \mathrm{kg})$, succinylcholine $(1.0 \mathrm{mg} / \mathrm{kg})$, and subsequent hourly slow injections of fentanyl $(0.025 \mathrm{mg} / \mathrm{kg}$ i.v. $)$ and continuous infusion of etomidate $(1.5 \mathrm{mg} / \mathrm{kg} / \mathrm{h})$. Dogs were ventilated with $30 \%$ oxygen in pressurized air to normocapnia. The body temperature was kept at $37^{\circ} \mathrm{C}$ with a heated water mattress. ECG standard lead II was continuously recorded. Under closed-chest conditions, LV and RV intracavitary pressures were recorded simultaneously with high-fidelity catheter-tip micromanometers introduced via the femoral artery and vein (Gaeltec Ltd, Dunvegan, UK and Millar Instruments Inc, Houston, TX, USA). In each animal a bolus injection of isoproterenol (ISO, $2.5 \mu \mathrm{g} / \mathrm{kg}$ ) was administered and repeated twice, with each next infusion given after baseline values had been stably reestablished.

\subsubsection{Cell-I solation Procedure}

Adult female beagle dogs were used for myocyte isolations. A total of 12 dogs were included in the study. Anesthesia was induced with $45 \mathrm{mg} / \mathrm{kg}$ pentobarbital. Once full anesthesia was reached, the chest was opened via a left thoracotomy and the heart was excised and placed in an $\mathrm{O}_{2}$-gassed, $\mathrm{Ca}^{2+}$-free standard buffer solution at approximately $4^{\circ} \mathrm{C}$. The cell-isolation procedure was the same as previously described ${ }^{33}$. Briefly, both the left-anterior-descending and right coronary arteries were cannulated and perfused simultaneously. After $\sim 20$ min of collagenase perfusion and subsequent washout of the enzyme, the epicardial surface layer was removed from wedges of both the LV and RV until a depth of $\geq 3 \mathrm{~mm}$ was reached. Softened tissue samples were collected from the midmyocardial layer underneath, while contamination with the endocardium was avoided. Samples were gently agitated, filtered and washed. Midmyocytes were stored at room temperature in standard buffer solution (vide infra) and only quiescent rod-shaped cells with clear cross-striations and without granulation were used for the experiments. Cells were used within $48 \mathrm{~h}$ of isolation. 


\subsubsection{Adenoviral I nfection of Dog Ventricular Myocytes}

Isolated cells were suspended in minimal essential medium (MEM: M 4780; Sigma, St Louis, Missouri, USA) containing $1.2 \mathrm{mM} \mathrm{Ca}{ }^{2+}, 2.5 \%$ fetal bovine serum (FBS, Invitrogen, Cergy-Pontoise, France), 1\% penicillin-streptomycin, 2\% HEPES (pH 7.6) and plated on $35 \mathrm{~mm}$, laminin-coated culture dishes $(10 \mu \mathrm{g} / \mathrm{mL}$ laminin, $2 \mathrm{~h})$ at a density of 104 cells per dish. Dishes were kept in an incubator $\left(95 \% \mathrm{O}_{2}, 5 \% \mathrm{CO}_{2}\right.$, $37^{\circ} \mathrm{C}$ ) for $2 \mathrm{~h}$. Then the medium was replaced by $400 \mu \mathrm{L}$ of FBS-free MEM containing adenoviruses encoding for the cytoplasmic CAMP sensor Epac2-camps ${ }^{47}$ at a multiplicity of infection (MOI) of 1000 pfu/cell, the plasma-membrane targeted pmEpac2-camps ${ }^{48}$ at a MOI of 700 pfu/cell and the cytoplasmic PKA sensor AKAR3NES ${ }^{49}$ at a MOI of $1000 \mathrm{pfu} / \mathrm{cell}$.

\subsubsection{I $\mathrm{CaL}$ Recordings}

The whole-cell configuration of the patch-clamp technique was used to record $I_{\text {CaL }}$. Patch-electrode resistance was between 1-2 $M \Omega$ when filled with internal solution containing (in $\mathrm{mM}$ ): $\mathrm{CsCl} 118, \mathrm{EGTA} \mathrm{5,} \mathrm{MgCl}_{2}$ 4, $\mathrm{Na}_{2}$ phosphocreatine 5, $\mathrm{Na}_{2}$ ATP 3.1, $\mathrm{Na}_{2} \mathrm{GTP} 0.42, \mathrm{CaCl}_{2} 0.062$ (pCa 8.5), HEPES 10, adjusted to pH 7.3 with $\mathrm{CsOH}$. Extracellular $\mathrm{Cs}^{+}$-Ringer solution contained (in $\mathrm{mM}$ ): $\mathrm{CaCl}_{2} 1.8, \mathrm{MgCl}_{2} 1.8, \mathrm{NaCl}$ 107.1, $\mathrm{CsCl} 20, \mathrm{NaHCO}_{3} 4, \mathrm{NaH}_{2} \mathrm{PO}_{4}$ 0.8, D-glucose 5, sodium pyruvate 5, HEPES 10, adjusted to $\mathrm{pH} 7.4$ with $\mathrm{NaOH}$. The cells were depolarized every $8 \mathrm{~s}$ from -50 to 0 $\mathrm{mV}$ during $400 \mathrm{~ms}$. The use of $-50 \mathrm{mV}$ as holding potential allowed the inactivation of voltage-dependent $\mathrm{Na}^{+}$currents. $\mathrm{K}^{+}$currents were blocked by replacing all $\mathrm{K}^{+}$ions with external and internal $\mathrm{Cs}^{+}$. Amplifiers RK-400 (Bio-Logic, Claix, France) or Axopatch 200B (Axon Instruments, Inc., Union City, CA, USA) were used for voltage clamping and acquisition. Currents were analogue filtered at $3 \mathrm{kHz}$ and digitally sampled at $10 \mathrm{kHz}$ using a 12-bit analogue-to-digital converter (DT2827; Data translation, Marlboro, Massachusetts, USA) connected to a compatible PC or a Digidata $1440 \mathrm{~A}$ interface connected to a computer equipped with pClamp 10 software (Axon Instruments, Inc., Union City, CA, USA). The maximal amplitude of whole-cell $I_{\text {CaL }}$ was measured as previously described ${ }^{50}$. Currents were not compensated for capacitance and leak currents.

\subsection{5 $\mathrm{I}_{\mathrm{Ks}}$ Recordings}

The whole-cell configuration of the patch-clamp technique was also used to record $\mathrm{I}_{\mathrm{Ks}}$. Patch electrodes had a resistance between 0.5-2 $\mathrm{M} \Omega$ when filled with internal solution containing (in $\mathrm{mM}$ ): $\mathrm{K}$-aspartate $125, \mathrm{KCl} 20, \mathrm{MgCl}_{2} 1.0, \mathrm{MgATP} 5$, HEPES 5 and EGTA 10, pH 7.2 with $\mathrm{KOH}$. The external solution had the following composition (mM): $\mathrm{NaCl} 145, \mathrm{KCl} 4.0, \mathrm{CaCl}_{2} 1.8, \mathrm{MgCl}_{2} 1.0$, glucose 11 and HEPES 10, $\mathrm{pH} 7.4$ with $\mathrm{NaOH}$ at $37^{\circ} \mathrm{C}$. During the recordings, $\mathrm{KCl}$ was omitted from the external solution to increase $\mathrm{I}_{\mathrm{Ks}}$ amplitude, which occurs through changes in the concentration 
gradient, while leaving the kinetics of $\mathrm{I}_{\mathrm{Ks}}$ activation and deactivation uninfluenced ${ }^{51}$. $\mathrm{I}_{\mathrm{CaL}}$ was blocked with nifedipine $(5 \mu \mathrm{M})$ and $\mathrm{I}_{\mathrm{Kr}}$ with dofetilide $(1 \mu \mathrm{M})$. The myocytes were depolarized from a holding potential of $-50 \mathrm{mV}$ to $+50 \mathrm{mV}$ for $3 \mathrm{~s}$, with the tail currents on repolarization to $0 \mathrm{mV}$ quantified as $\mathrm{I}_{\mathrm{ks}}$. Ten baseline pulses were recorded followed by 3 pulses during brief $(15 \mathrm{~s}$ ) stimulation with ISO (plus or minus PDE inhibitor) and this was followed by a further 50 pulses during perfusion with 0 $\mathrm{mM} \mathrm{K}^{+}$( \pm PDE inhibitor). Fast solution changes were achieved with the complete VC6 fast-step perfusion system (Harvard Apparatus, Holliston, MA, USA) together with a multibarrel glass pipette that was positioned $\sim 50 \mu \mathrm{m}$ from the cell during drug application.

\subsubsection{Recordings of $\mathrm{Ca}^{2+}$ Transients and Sarcomere Shortening}

Myocytes were loaded with $5 \mu \mathrm{M}$ Fura-2 AM (Invitrogen) during $15 \mathrm{~min}$ at room temperature and then washed with Ringer solution containing (in $\mathrm{mM}$ ): $\mathrm{NaCl}$ 121.6, $\mathrm{KCl}$ 5.4, $\mathrm{MgCl}_{2}$ 1.8, $\mathrm{CaCl}_{2}$ 1.8, $\mathrm{NaHCO}_{3} 4, \mathrm{NaH}_{2} \mathrm{PO}_{4}$ 0.8, D-glucose 5, Na-pyruvate 5, HEPES 10, adjusted to $\mathrm{pH} 7.4$ with $\mathrm{NaOH}$. The loaded cells were field-stimulated $(5 \mathrm{~V}, 4 \mathrm{~ms}$ ) at a frequency of $0.3 \mathrm{~Hz}$. Then, sarcomere length and Fura-2 ratio (measured at $512 \mathrm{~nm}$ upon excitation at $340 \mathrm{~nm}$ and $380 \mathrm{~nm}$ ) were simultaneously recorded using an IonOptix System (IonOptix, Milton, MA, USA). Cell contraction was assessed as the percentage of sarcomere shortening, which is the ratio of twitch amplitude (difference of end-diastolic and peak systolic sarcomere lengths) to end-diastolic sarcomere length. $\mathrm{Ca}^{2+}$-transient amplitude was assessed by the percentage of variation of the Fura-2 ratio, by dividing the twitch amplitude (difference of end-diastolic and peak systolic ratios) to end-diastolic ratio. All parameters were calculated offline with dedicated software (IonWizard 6x, IonOptix).

\subsubsection{FRET Measurements}

The same Ringer solution as described above for $\mathrm{Ca}^{2+}$ transients and cell shortening measurements was used in these experiments. Images were captured every $5 \mathrm{~s}$ using the $40 \mathrm{x}$ oil immersion objective of a Nikon TE 300 inverted microscope connected to a software-controlled (Metafluor, Molecular Devices, Sunnyvale, California, USA) cooled charge coupled (CCD) camera (Sensicam PE; PCO, Kelheim, Germany). CFP was excited during $150-300$ ms by a Xenon lamp (100 W, Nikon, Champigny-sur-Marne, France) using a 440/20BP filter and a 455LP dichroic mirror. Dual-emission imaging of CFP and YFP was performed using an Optosplit II emission splitter (Cairn Research, Faversham, UK) equipped with a 495LP dichroic mirror and BP filters 470/30 and 535/30, respectively. A region of interest including the entire cell was used for measurement of average CFP and YFP intensity. CFP and YFP intensities were background corrected and the YFP emission was corrected for CFP bleed through. The ratio of CFP over corrected YFP was used as an index of CAMP concentration. 


\subsubsection{Reagents}

Cilostamide was from Tocris Bioscience (Bristol, UK) and Ro 20-1724 was from Calbiochem (Darmstadt, Germany). Unless specified, all other drugs were from Sigma (Saint Quentin, France). Isoproterenol (ISO) was first dissolved in distilled water containing $30 \mu \mathrm{M}$ ascorbic acid and then kept in the dark at $4^{\circ} \mathrm{C}$ until use.

\subsubsection{Data Analysis and Statistics}

All results are expressed as mean \pm SEM. For statistical evaluation the paired and unpaired Student's t-test were used, and a difference was considered statistically significant when $\mathrm{P}$ was $<0.05$.

\subsection{Results}

\subsubsection{In Vivo I notropic Response to I soproterenol in RV and LV}

Pressure recordings were obtained simultaneously from the RV and LV at baseline and during infusion of ISO in anesthetized beagle dogs. Representative examples relative to the ECG are shown in Figure 1A. At baseline, systolic pressures and $\mathrm{dP} / \mathrm{dt}_{\max }$ were significantly higher in the LV than in the RV (Figure 1B). For example, $\mathrm{dP} / \mathrm{dt}_{\text {max }}$ was $2582 \pm 194 \mathrm{~mm} \mathrm{Hg} / \mathrm{s}$ in the LV versus $534 \pm 45 \mathrm{~mm} \mathrm{Hg} / \mathrm{s}$ in the RV $(\mathrm{P}<0.001)$. Upon ISO, $\mathrm{dP} / \mathrm{dt}_{\max }$ increased $\sim 3.4$-fold in the LV compared to almost 5fold in the RV $(P<0.05$; Figure 1B, left panel). Accordingly, the relative increase in systolic pressure by $\beta A R$ stimulation was significantly higher in the RV than LV, reaching on average $66 \pm 19 \mathrm{~mm} \mathrm{Hg}(+61 \pm 6 \%)$ and $172 \pm 31 \mathrm{~mm} \mathrm{Hg}(+20 \pm 4 \%)$, respectively $(\mathrm{P}<0.01)$ during ISO (Figure 1B, right panel). Complete recovery from the positive inotropic response (Figure 1C) occurred faster in the LV than the RV after the ISO bolus infusions (Figure 1C, right panel). Diastolic pressures did not show discernable interventricular differences from baseline to ISO. 
A
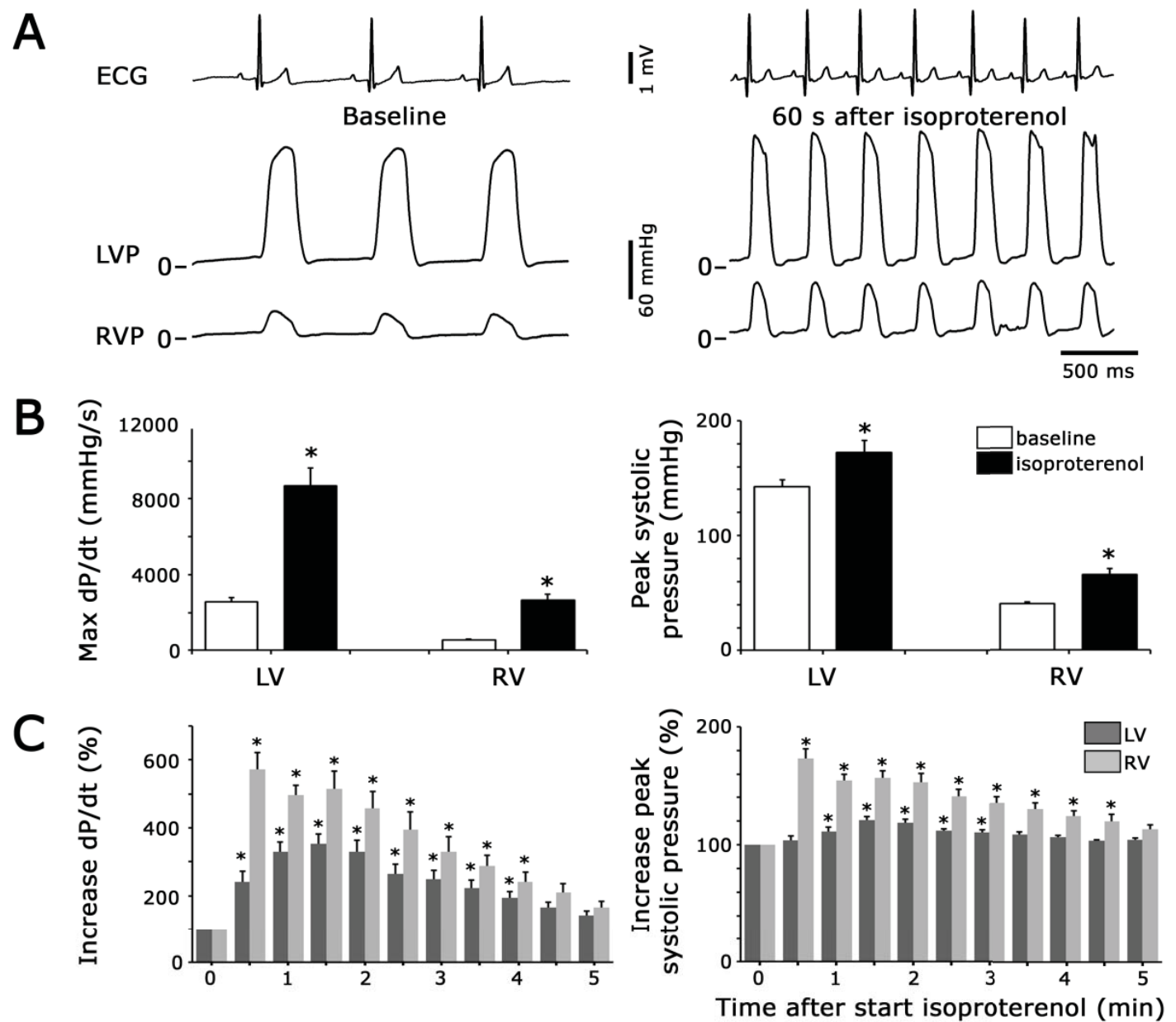

Figure 1: Differential response to $\beta A R$ stimulation in the LV versus RV in anesthetized dogs A. Representative pressure recordings, together with ECG lead II, before and $60 \mathrm{~s}$ after bolus infusion of the $\beta A R$ agonist ISO at $2.5 \mu \mathrm{g} / \mathrm{kg}$. B. Bar graphs showing average values ( $n=4$ dogs) for $\mathrm{dP} / \mathrm{dt}_{\max }$ and peak systolic pressures in the LV and RV before and after infusion of ISO. C. Bar graphs illustrating the average time courses of alterations in $\mathrm{dP} / \mathrm{dt}_{\max }$ and peak systolic pressures after infusion of ISO, including recovery phase. Data are shown as change from baseline $*, \mathrm{P}<0.05$.

\subsubsection{Sarcomere Shortening and $\mathrm{Ca}^{2+}$-Transient Measurements in Response to $\beta A R$ Stimulation in RV and LV Midmyocytes}

Sarcomere shortening and $\mathrm{Ca}^{2+}$ transients were simultaneously measured in Fura-2loaded RVMs and LVMs obtained from the same hearts and paced at $0.3 \mathrm{~Hz}$. There was no significant difference in basal sarcomere length, basal sarcomere shortening, basal Fura-2 ratio and basal $\mathrm{Ca}^{2+}$-transient amplitude (Table 1). As shown by the individual traces of Figure $\mathbf{2 A}$ and $\mathbf{2 B}$, a pulse application of ISO (100 nM, $15 \mathrm{~s}$ ) increased the amplitude of sarcomere shortening and $\mathrm{Ca}^{2+}$ transient in both LVMs and RVMs, but these effects were exacerbated in RVMs. Indeed, on average ISO increased sarcomere shortening by $\sim 25$-fold in RVMs versus $\sim 10$ fold in LVMs and increased $\mathrm{Ca}^{2+}$ transients 3-fold in RVMs versus 2-fold in LVMs (Figure 2C and 2D). 
Table 1

\begin{tabular}{|lcccc|}
\hline & RVMs & N & LVMs & n \\
\hline Contraction & & & & \\
$\mathrm{SL}(\mu \mathrm{m})$ & $1.74 \pm 0.01$ & 20 & $1.72 \pm 0.01$ & 18 \\
$\Delta \mathrm{L}(\%)$ & $1.30 \pm 0.27$ & 20 & $1.23 \pm 0.20$ & 18 \\
\hline Calcium & & & & \\
Fura-2 ratio & $4.93 \pm 0.28$ & 20 & $4.66 \pm 0.27$ & 18 \\
$\Delta \mathrm{R}(\%)$ & $8.31 \pm 0.84$ & 20 & $9.00 \pm 1.01$ & 18 \\
\hline Electrophysiology & & & & \\
Cell capacitance $(\mathrm{pF})$ & $132.9 \pm 3.7$ & 89 & $123.0 \pm 3.5$ & 92 \\
$\mathrm{I}_{\mathrm{CaL}}$ density $(\mathrm{pA} / \mathrm{pF})$ & $2.9 \pm 0.2$ & 89 & $3.3 \pm 0.2$ & 92 \\
$\mathrm{I}_{\mathrm{Ks}}$ density $(\mathrm{pA} / \mathrm{pF})$ & $1.1 \pm 0.2$ & 18 & $0.8 \pm 0.1(\mathrm{P}<0.05)$ & 13 \\
\hline
\end{tabular}

Table 1: Basal parameters measured in RVMs and LVMs. The effect of ISO lasted also longer in RVMs than in LVMs, as indicated by the time to half-maximal recovery $\left(t_{1 / 2 \text { off }}\right)$ of sarcomere shortening (79 $\pm 11 \mathrm{~s}$ in RVMs versus $54 \pm 3 \mathrm{~s}$ in LVMs) and $\mathrm{Ca}^{2+}$ transient $(82 \pm 7 \mathrm{~s}$ in RVMs versus $49 \pm 6 \mathrm{~s}$ in LVMs). These results indicate that the differential in vivo positive inotropic effects by ISO have a cellular origin.

\subsection{3 $\beta$ AR Regulation of Cytoplasmic CAMP Signals and PKA Activity in RV and LV Midmyocytes}

Since $\beta A R$ stimulation of ECC involves CAMP mobilization, this second messenger was measured in intact LVMs and RVMs by fluorescence resonance energy transfer (FRET) using the cytoplasmic sensor Epac2-camps ${ }^{47}$. Figure 3A shows pseudocolor images of a LVM and a RVM expressing Epac2-camps. At 1 min after application of ISO (100 nM, $15 \mathrm{~s})$, the CFP/YFP ratio increased to a greater extent in RVMs than in LVMs. As shown in Figure 3B, ISO led to a transient increase in the CFP/YFP ratio that was on average $\sim 2$-fold higher in RVMs versus LVMs $(P<0.001)$ reflecting enhanced cytoplasmic cAMP accumulation in RVMs. Because PKA is the primary target of CAMP for short-term regulation of ECC, cytoplasmic PKA activity was monitored using the PKA FRET sensor AKAR3-NES ${ }^{49}$. As shown in the illustrative pseudocolor image of Figure $\mathbf{3 C}$ and on the average time course of Figure 3D, although the maximal increase in YFP/CFP ratio was identical at $1 \mathrm{~min}$, the signal decayed faster in LVMs than RVMs and became significantly higher in RVMs than LVMs after 2 min. This indicates that cytoplasmic PKA activity was higher in RVMs than in LVMs in response to BAR stimulation. 
A

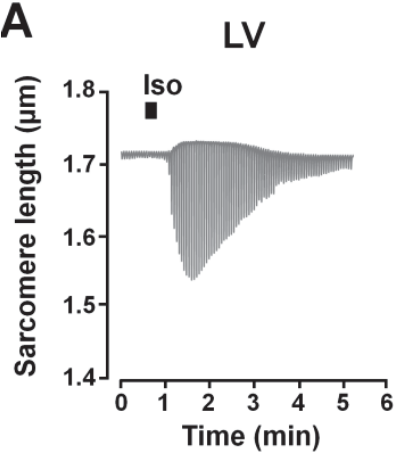

B

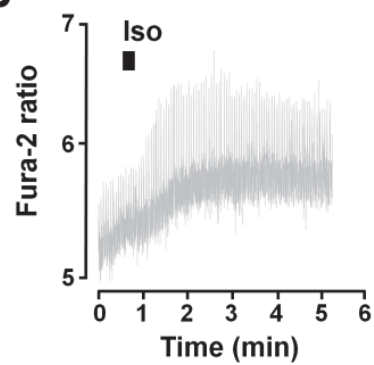

RV
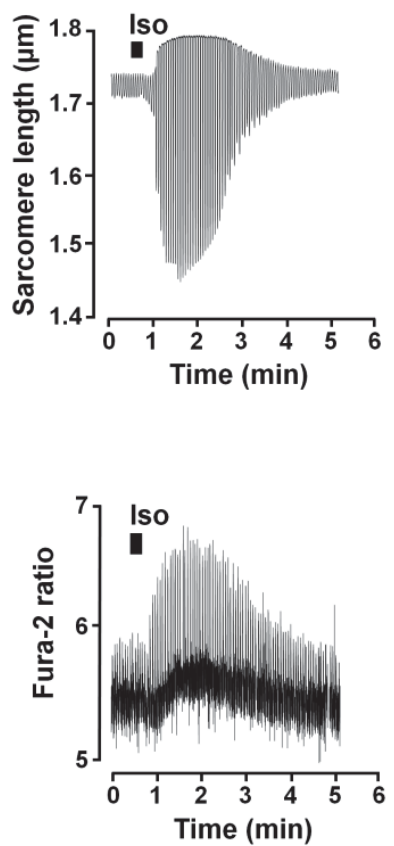

C

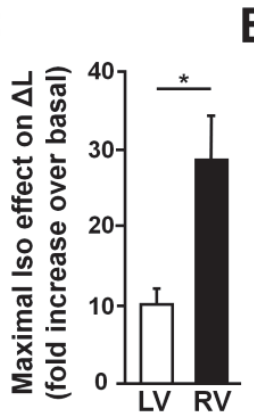

E

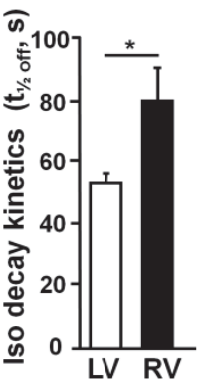

D

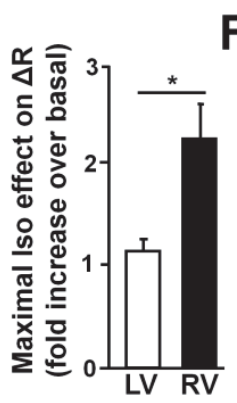

$F$ क

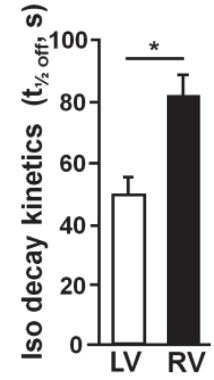

Figure 2: $\beta A R$ Regulation of ECC in LV and RV Midmyocytes (A, B) Raw traces of sarcomere shortening $\mathbf{A}$. and $\mathrm{Ca}^{2+}$ transients $\mathbf{B}$. recorded in Fura-2 loaded LVMs and RVMs paced at 0.3 $\mathrm{Hz}$. The solid bar indicates application of isoproterenol (ISO, $100 \mathrm{nM}, 15 \mathrm{~s}$ ). (C, D) Average maximal effect of ISO on sarcomere shortening $\mathbf{C}$. and $\mathrm{Ca}^{2+}$ transients amplitude $\mathbf{D}$. in LVMs $(n=10)$ and RVMs $(n=12)$. E, F. Decay kinetics of the $\beta A R$ response estimated by the time to $50 \%$ decrease $\left(\mathrm{t}_{1 / 2 \text { off }}\right.$ ) of sarcomere-shortening amplitude $\mathbf{E}$. and $\mathrm{Ca}^{2+}$-transient amplitude $\mathbf{F}$. The bar graphs indicate the mean+SEM. Statistically significant differences between LVMs and RVMs are indicated by *, $\mathrm{P}<0.05$.

The lack of difference in the YFP/CFP ratio at the beginning of the stimulation was most likely due to saturation of the AKAR3-NES sensor at this concentration of ISO, as indicated by the lack of a sharp peak in RVMs and by previous experiments in rat ventricular myocytes showing that a $40 \%$ change in YFP/CFP represents the maximal FRET change that can be obtained with this sensor (data not shown). 
A

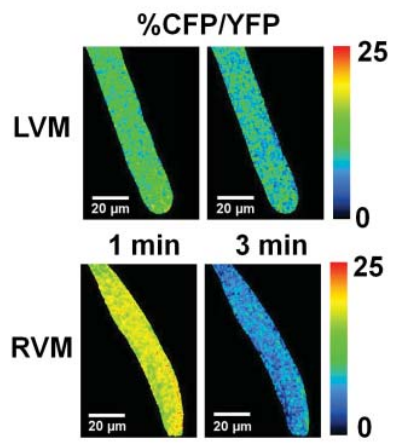

C

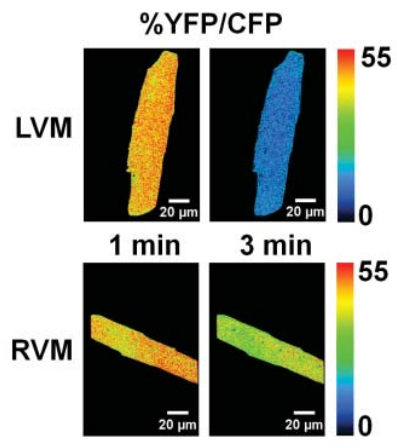

B

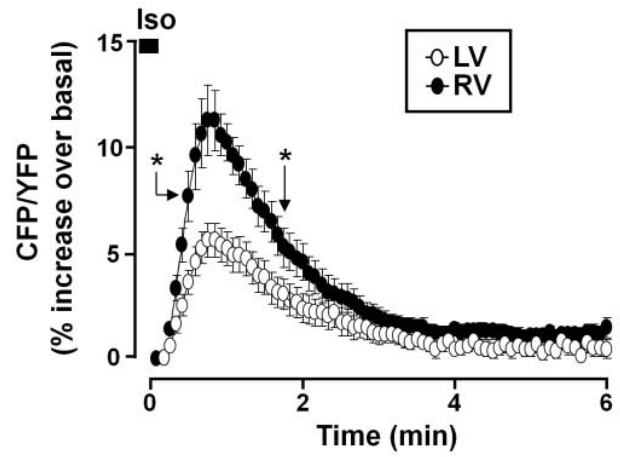

D

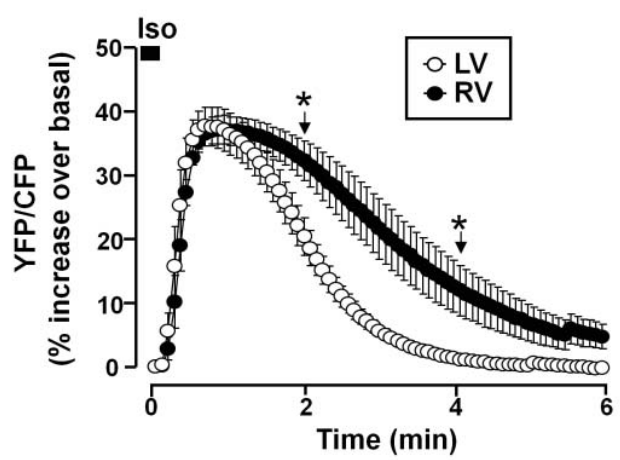

Figure 3: RV Midmyocytes Display Enhanced cAMP Accumulation and PKA Activity in Response to $\beta A R$ Stimulation. A. Pseudocolor images of a dog LVM (upper images) and a dog RVM (lower images) expressing the cytoplasmic CAMP FRET sensor Epac2-camps. Illustrated are the percent increases of the CFP/YFP ratio over baseline at $1 \mathrm{~min}$ and at $3 \mathrm{~min}$ after ISO (100 nM, 15 s) stimulation. B. Average cytoplasmic cAMP accumulation elicited by ISO pulse stimulation in RVMs $(n=36)$ and LVMs $(n=37)$. C. Pseudocolor images of a dog LVM (upper images) and a dog RVM (lower images) expressing the cytoplasmic PKA sensor AKAR3-NES. Illustrated is the percent increase of the YFP/CFP ratio over basal at $1 \mathrm{~min}$ and at $3 \mathrm{~min}$ after the ISO pulse stimulation. D. Average PKA activation elicited by ISO pulse stimulation in RVMs $(n=5)$ and LVMs $(n=4)$. Statistically significant differences between LVMs and RVMs are indicated by $*, P<0.05$.

\subsection{4 $\beta$ AR Regulation of Subsarcolemmal CAMP, $I_{\mathrm{CaL}}$ and $\mathrm{I}_{\mathrm{KS}}$ in RV and LV Midmyocytes}

Because subsarcolemmal CAMP plays an important role in ECC, we next used a plasma-membrane targeted version of Epac2-camps (pmEpac2-camps ${ }^{48}$ ) to monitor CAMP specifically in this compartment. To our surprise, comparison of LVMs and RVMs revealed no difference in subsarcolemmal cAMP ([CAMP]pm) generated by $\beta A R$ stimulation with an ISO pulse. Indeed, neither the maximal cAMP elevation nor its decay kinetics was different between LVMs and RVMs (Figure 4A). We next wondered whether the same was true for the major sarcolemmal targets of the $\beta A R / C A M P / P K A$ pathway, $I_{\text {Cat }}$ and $I_{K s}$. As shown in Table 1, there was no difference in basal $\mathrm{I}_{\text {Cat }}$ density between RVMs and LVMs. A pulse stimulation with ISO 
(100 nM, $15 \mathrm{~s}$ ) resulted in a similar transient increase of $\mathrm{I}_{\mathrm{CaL}}$ between LVMs and RVMs, both in terms of amplitude and duration (Figure 4B). In contrast to $\mathrm{I}_{\text {CaL, }}$ basal $\mathrm{I}_{\mathrm{Ks}}$ density was higher in RVMs than LVMs (Table 1), which is consistent with earlier findings (33). However, pulse $\beta A R$ stimulation increased $I_{K s}$ to similar degrees in LVMs and RVMs (Figure 4C).

A
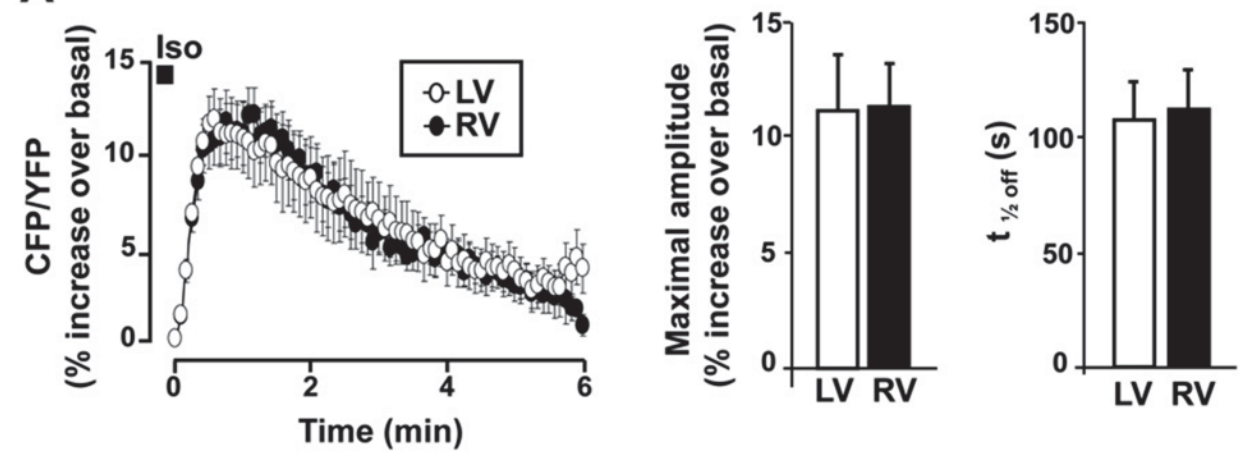

B
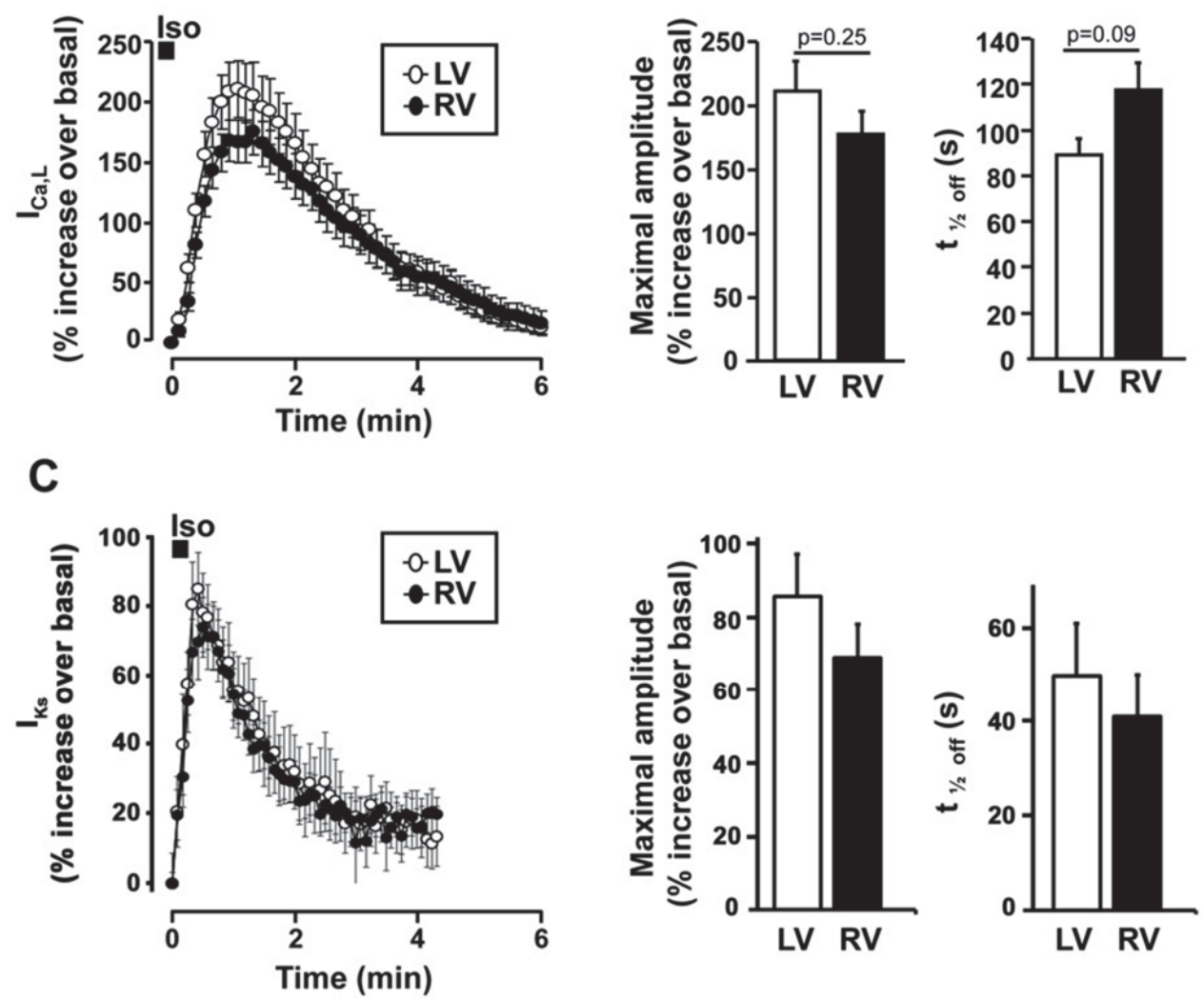

Figure 4: $\beta A R$ Regulation of Subsarcolemmal cAMP, $I_{\text {CaL }}$ and $I_{K s}$ in LV and RV Midmyocytes A. Left, average time-courses of ISO (100 nM, 15 s)-induced subsarcolemmal cAMP signals measured with pmEpac2-camps in RVMs $(n=13)$ and LVMs $(n=7)$. Right, bar graphs comparing average maximal amplitude and time to $50 \%$ decrease $\left(t_{1 / 2 \text { off }}\right)$ of the CAMP response. B. Left, average time-courses of $\mathrm{I}_{\text {Cal }}$ following ISO $(100 \mathrm{nM}, 15 \mathrm{~s})$ stimulation in RVMs $(n=22)$ and LVMs $(n=25)$. Right, bar graphs comparing average maximal amplitude and time to $50 \%$ decrease $\left(t_{1 / 2 \text { off }}\right)$ of the $I_{\text {CaL }}$ response. C. Left, average time-course of $I_{K s}$ response to ISO (100 nM, $15 \mathrm{~s})$ in RVMs $(n=8)$ and LVMs $(n=8)$ Right, bar graphs comparing average maximal amplitude and time to $50 \%$ decrease ( $\left.\mathrm{t}_{1 / 2 \mathrm{off}}\right)$ of the $\mathrm{I}_{\mathrm{KS}}$ response. 
These results indicate that i) differences in BAR CAMP signals between LVMs and RVMs are compartment-specific and ii) the increased stimulation of ECC by ISO in RVMs does not involve differential regulation of $\mathrm{I}_{\mathrm{CaL}}$, and $\mathrm{I}_{\mathrm{Ks}}$.

PDE3 and PDE4 shape distinct $\beta A R$ CAMP signals in RVMs and LVMs. We next investigated whether phosphodiesterases (PDEs) are involved in the differences in cytoplasmic CAMP accumulation observed between RVMs and LVMs upon BAR stimulation. We first tested the implication of PDE3, because of its critical importance for the control of cardiac contractility in dog ${ }^{21}$. Thus, myocytes expressing Epac2-camps were challenged with ISO (100 nM, $15 \mathrm{~s})$ in the presence of the specific PDE3 inhibitor cilostamide (Cil, $1 \mu \mathrm{M})$, and the inhibitor was maintained during ISO washout. Figure 5A shows that Cil doubled the maximal amplitude of the ISO-induced cAMP transient in LVMs, while having little effect in RVMs. Cil also profoundly impaired cAMP recovery in both LVMs and RVMs, as indicated by the $\sim 3$-fold increase in time to half maximal decay ( $t_{1 / 2 \text { off }}$ ) values (Figure 5A). We next tested the implication of PDE4, which represents the major soluble CAMP-PDE activity in dog ventricle ${ }^{18}$ by using the specific PDE4 inhibitor Ro-201724 (Ro, $10 \mu \mathrm{M}$ ). Figure 5B shows that Ro induced a 3-fold increase in the amplitude of the cAMP transient elicited by ISO in LVMs and a 2-fold increase in RVMs. PDE4 inhibition also significantly delayed cAMP recovery, as demonstrated by a $\sim 2.5$-fold increase in time-to-half-maximal decay $\left(t_{1 / 2 \text { off }}\right)$ values. Thus, PDE3 and PDE4 regulate cytoplasmic CAMP accumulation upon $\beta A R$ stimulation in canine ventricular midmyocytes. However, this control is less stringent in RVMs compared to LVMs, resulting in higher cytoplasmic CAMP ([CAMP]cyt) upon $\beta A R$ stimulation.

Regulation of membrane CAMP, $\mathrm{I}_{\mathrm{CaL}}$ and $\mathrm{I}_{\mathrm{Ks}}$ by PDE3 and PDE4 in RVMs and LVMs. Given the large contribution of PDE4 to cytoplasmic CAMP hydrolysis upon BAR stimulation, we compared the respective roles of PDE3 and PDE4 in the BAR regulation of subsarcolemmal CAMP in canine ventricular myocytes. As shown in Figure 6A, inhibition of PDE3 with $1 \mu \mathrm{M}$ Cil had no effect on the maximal CAMP elevation at the plasma membrane but delayed cAMP recovery in LVMs and RVMs.

Interestingly, the difference in average $t_{1 / 2 \text { off }}$ values between ISO and ISO+Cil reached statistical significance only in LVMs ( $\mathrm{t}_{1 / 2 \text { off }}$ was $107 \pm 17 \mathrm{~s}$ for ISO, and $232 \pm 50 \mathrm{~s}$ for ISO+Cil, $\mathrm{P}<0.05$ whereas in RVMs, $\mathrm{t}_{1 / 2 \text { off }}$ was $112 \pm 17 \mathrm{~s}$ for ISO alone and $171 \pm 39 \mathrm{~s}$ for ISO+Cil, $\mathrm{p}=0.36$ ) suggesting a stronger contribution of membrane-bound PDE3 in LVMs versus RVMs to CAMP hydrolysis upon BAR stimulation. In sharp contrast to what was observed in the cytoplasm, PDE4 inhibition with Ro at $10 \mu \mathrm{M}$ had no effect, neither on the amplitude nor on the recovery kinetics of the CAMP transient generated by ISO pulse stimulation at the plasma membrane (Figure 6B). We next investigated the respective contribution of PDE3 and PDE4 to the regulation $\mathrm{I}_{\mathrm{CaL}}$ and $\mathrm{I}_{\mathrm{Ks}}$. 
A
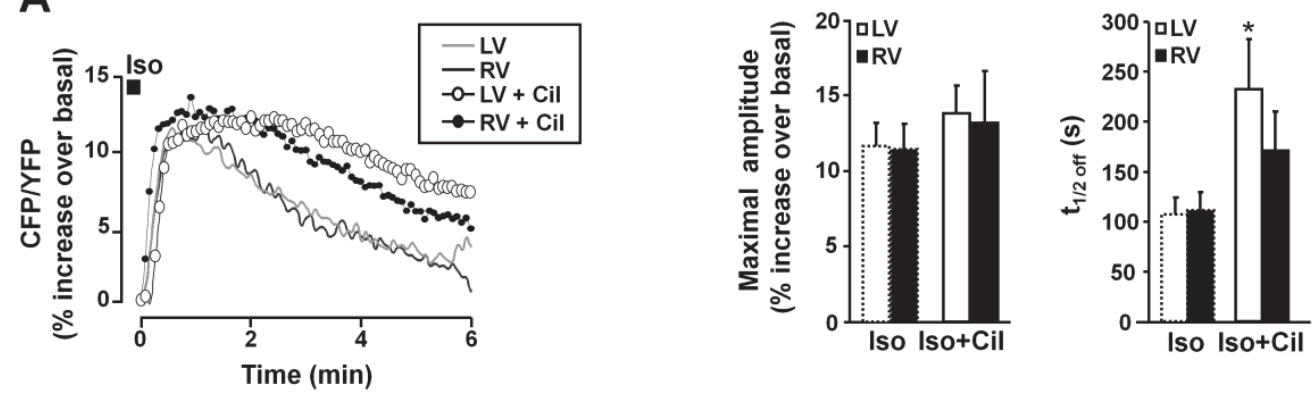

B
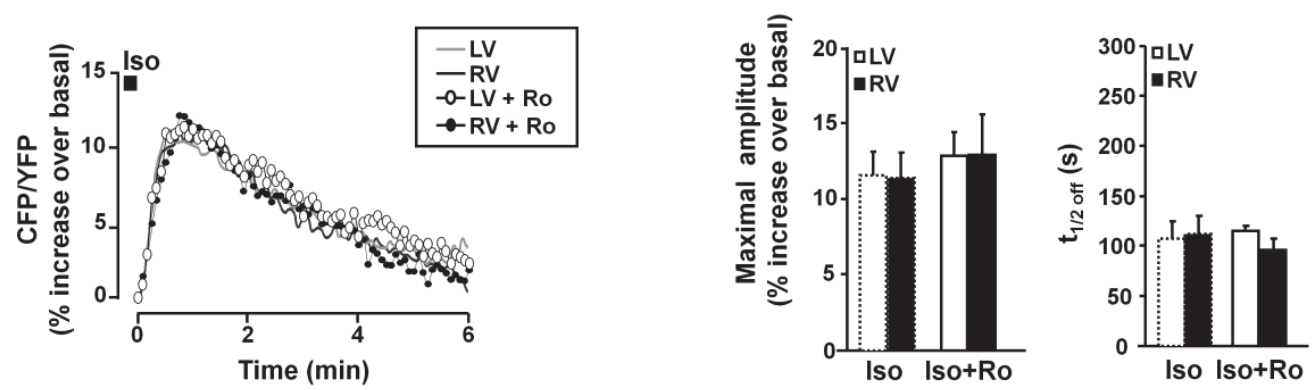

Figure 5: Regulation of $\beta A R$ Cytoplasmic cAMP Signals by PDE3 and PDE4 in LVMs and RVMs A. Left, average time course of cAMP levels measured in the cytoplasm with Epac2-camps following BAR stimulation with ISO (100 nM, $15 \mathrm{~s}$ ) alone (gray and black lines, data from Figure $3 \mathrm{~B}$ ) or during administration of the PDE3 inhibitor cilostamide (Cil, $1 \mu \mathrm{M}$, white circles: LVMs, $n=13$; black circles: RVMs, $n=17$ ). Right, Bar graphs representing the average maximal amplitude and time to $50 \%$ recovery ( $\mathrm{t}_{1 / 2 \mathrm{off}}$ ) of the cytoplasmic cAMP transients induced by ISO alone or ISO with Cil in LVMs $(n=13)$ and RVMs $(n=17)$. B. Left, average time course of cytosolic cAMP levels following BAR stimulation with Iso (100 nM, $15 \mathrm{~s})$ alone or during administration of the PDE4 inhibitor Ro 20-1724 (Ro, $10 \mu \mathrm{M}$; white circles: LVMs, $\mathrm{n}=21$; black circles: RVMs, $n=24)$. Right, bar graphs representing the average maximal amplitude and time to $50 \%$ recovery ( $\mathrm{t}_{1 / 2 \mathrm{off}}$ ) of the CAMP transients induced by Iso alone or ISO with Ro in LVMs $(n=21)$ and LVMs $(n=24)$. PDE inhibitors were added to the Iso solution and in the washout solution. Symbols and bar graphs indicate the mean \pm SEM. Statistical difference between ISO alone and ISO+PDE inhibitor in LVMs and RVMs is indicated as *, P<0.05; ***, $\mathrm{P}<0.001$. 
A
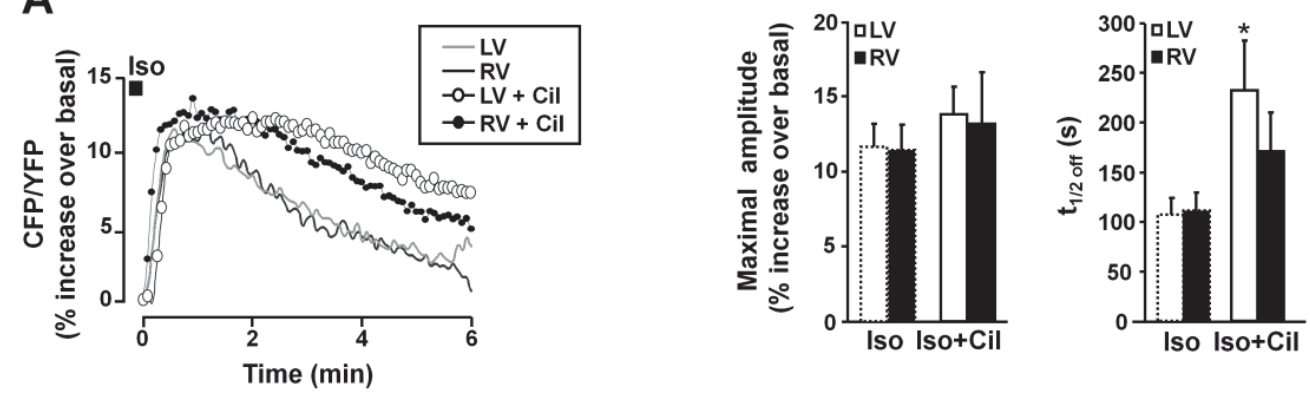

B
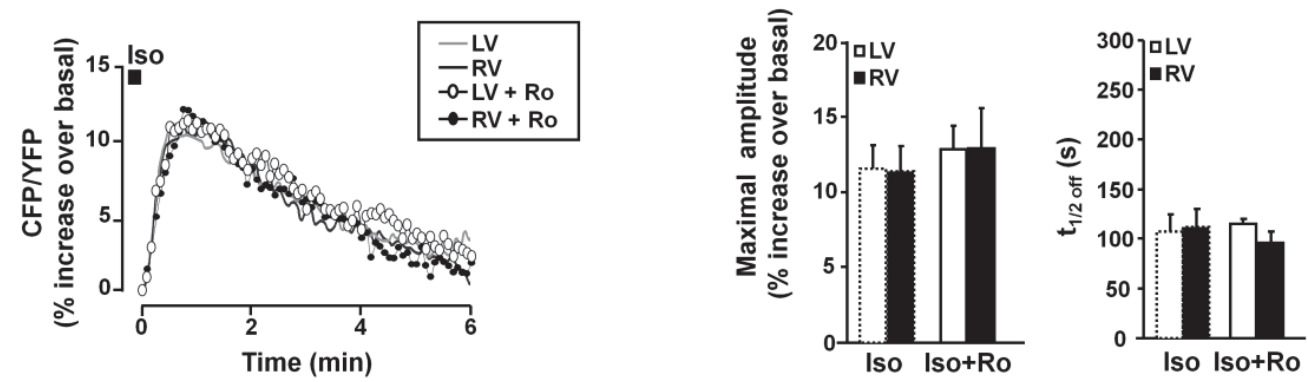

Figure 6: Regulation of Subsarcolemmal CAMP by PDE3 and PDE4 after brief $\beta A R$ Stimulation A. Left, average time course of CAMP levels measured at the plasma membrane with pmEpac2-camps following $\beta A R$ stimulation with ISO (100 nM, $15 \mathrm{~s}$ ) alone (gray and black lines, data from Figure 4A) and representative examples of the effect of the PDE3 inhibitor cilostamide (Cil, $1 \mu \mathrm{M})$ in a LVM (white circles) and a RVM (black circles). Right, bar graphs representing the average maximal amplitude and time to $50 \%$ recovery ( $\left.t_{1 / 2 \text { off }}\right)$ of the sarcolemmal cAMP transients induced by ISO alone or ISO with Cil in LVMs $(n=5)$ and RVMs $(n=5)$. B. Left, average time course of the plasma membrane cAMP levels following BAR stimulation with ISO (100 nM, $15 \mathrm{~s}$ ) alone (gray and black lines, data from Figure 4A) and representative examples of the effect of the PDE4 inhibitor Ro 20-1724 (Ro, $10 \mu \mathrm{M}$ ) in a LVM (white circles) and a RVM (black circles). Right, bar graphs representing the average maximal amplitude and time to $50 \%$ recovery ( $\mathrm{t}_{1 / 2 \text { off }}$ ) of the cAMP transients induced by ISO alone or ISO with Ro in LVMs $(n=9)$ and LVMs $(n=5)$. PDE inhibitors were added to the ISO solution and in the washout solution. Symbols and bar graphs indicate the mean \pm SEM. Statistical difference between ISO alone and ISO+PDE inhibitor in LVMs and RVMs is indicated as *, $\mathrm{P}<0.05$.

As shown in Figure 7A, PDE3 inhibition had no effect on the maximal $I_{\text {CaL }}$ stimulation induced by ISO (100 nM, $15 \mathrm{~s})$, but significantly delayed $\mathrm{I}_{\text {CaL }}$ recovery in LVMs and RVMs (in LVMs, $t_{1 / 2 \text { off }}$ was $91 \pm 7 \mathrm{~s}$ for ISO, and 203 $\pm 22 \mathrm{~s}$ for Iso+Cil, $\mathrm{P}<0.001$; in RVMs, $\mathrm{t}_{1 / 2 \text { off }}$ was $113 \pm 11 \mathrm{~s}$ for ISO alone and $176 \pm 19 \mathrm{~s}$ for ISO+Cil, $\mathrm{P}<0.05)$. Interestingly, PDE3 inhibition also slowed $\mathrm{I}_{\mathrm{Ks}}$ recovery in RVMs and LVMs (data not shown). In contrast, inhibition of PDE4 with Ro at $10 \mu \mathrm{M}$ did not modify $\mathrm{I}_{\mathrm{CaL}}$ (Figure $6 \mathrm{~B}$ ) nor $\mathrm{I}_{\mathrm{Ks}}$ recovery. 
A

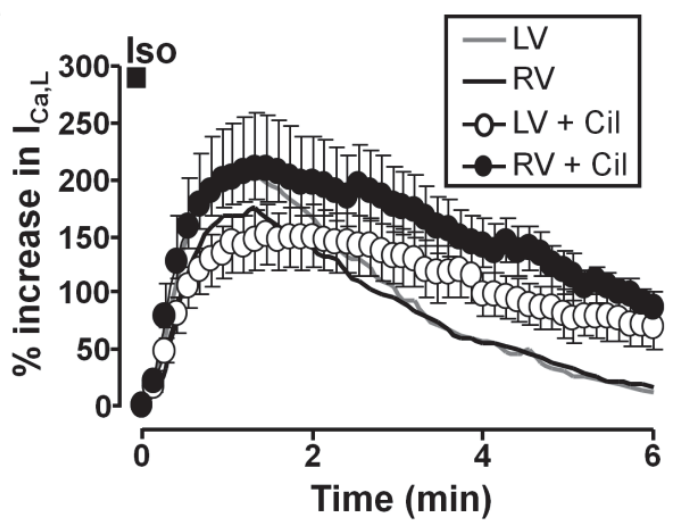

B

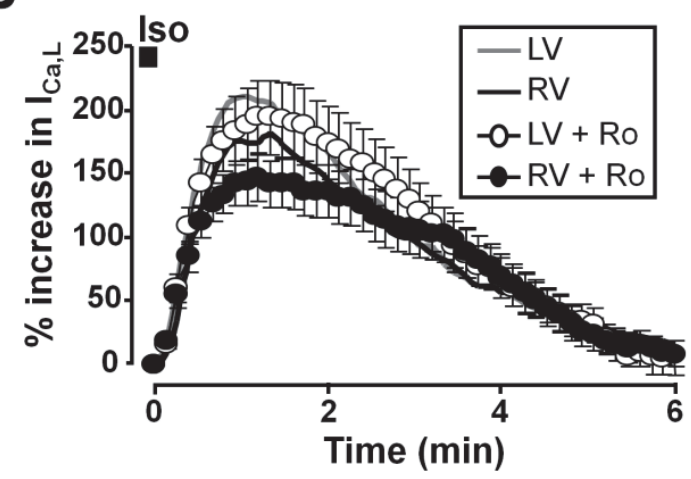

C

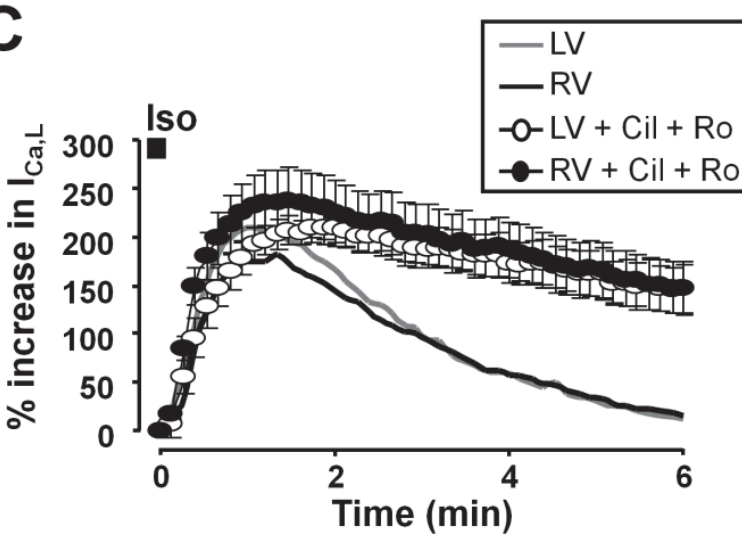

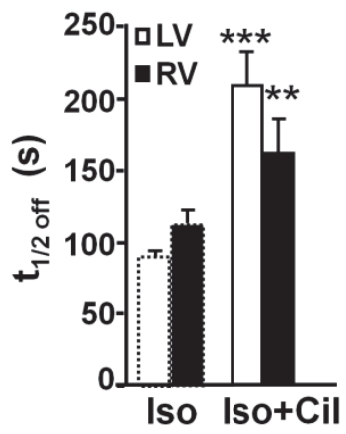
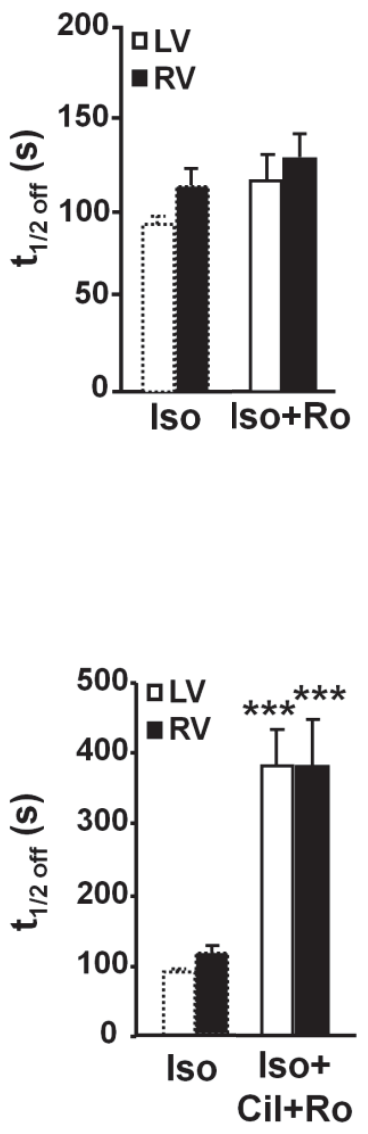

Figure 7: Regulation of $I_{C a L}$ by PDE3 and PDE4 after brief $\beta A R$ stimulation. Average time course of $\mathrm{I}_{\mathrm{CaL}}$ (left) and mean values of time for $50 \%$ recovery ( $\mathrm{t}_{1 / 2 \text { off }}$ ) (right) in response to $\beta$ AR stimulation with ISO (100 nM, $15 \mathrm{~s}$ ) alone or with A. cilostamide (Cil, $1 \mu \mathrm{M}$; white circles: LVMs $\mathrm{n}=18$; black circles: RVMs, $\mathrm{n}=11$ ); B. Ro 20-1724 (Ro, $10 \mu \mathrm{M}$; white circles: RVMs, $\mathrm{n}=15$; black circles: LVMs, $\mathrm{n}=11$ ) and $\mathbf{C}$. the combination of Cil and Ro (Cil+ Ro, white circles: LVMs, $n=11$; black circles: LVMs, $n=11$ ). PDE inhibitors were added to the ISO solution and in the washout solution. The symbols and bar graphs indicate the mean \pm SEM. Statistical difference between ISO alone and ISO+PDE inhibitor in LVMs and RVMs is indicated as $* *, \mathrm{P}<0.01 ; * * *, \mathrm{P}<0.001$. 
However, when PDE3 was inhibited by Cil, concomitant inhibition of PDE4 drastically prolonged the $\beta A R$ stimulation of the current (Figure $6 C$ ), with $t_{1 / 2 \text { off }}$ values reaching $381 \pm 66 \mathrm{~s}$ in RVMs $(\mathrm{P}<0.05$ versus ISO+Cil) and $381 \pm 51 \mathrm{~s}$ in LVMs $(P<0.01$ versus ISO+Cil). These results indicate that although PDE3 is dominant for $\beta A R$ regulation of $\mathrm{I}_{\mathrm{CaL}}$ in canine myocytes, PDE4 becomes important when PDE3 is inhibited.

\subsection{Discussion}

This study provides direct evidence that canine RV and LV differ in their sensitivity to $\beta A R$ stimulation due to intrinsic postsynaptic differences in myocyte $\beta A R$ signaling. The increased positive inotropic effect of ISO observed in the RV in vivo is correlated with enhanced efficiency of ISO to stimulate $\mathrm{Ca}^{2+}$ transients and sarcomere shortening in RVMs compared to LVMs isolated from the same hearts. Enhanced $\beta A R$ responsiveness in RVMs is not associated with modifications of $\beta A R$ signaling at the plasma membrane, since $\beta A R$ stimulation of subsarcolemmal [CAMP]i and of membrane currents $I_{C a L}$ and $I_{K s}$ are similar in RVMs and LVMs. However, RVMs display a higher cAMP accumulation and PKA activity in the cytoplasm compared to LVMs, and the difference in CAMP is abolished by inhibition of PDE3 and PDE4. Thus, PDE3 and PDE4 shape distinct compartmentalized CAMP signals that underlie regionally-specific differences in the cardiac ventricles upon $\beta A R$ stimulation.

Recent clinical studies indicate that during intense physical exercise, the afterload of the RV increases more than that of the LV ${ }^{41,52}$. Because the sympathetic system is strongly activated during exercise, these observations suggest that the RV could be more sensitive to sympathetic stimulation than the LV in humans. Earlier studies that compared the responsiveness of the LV versus RV to sympathetic nerve stimulation in dog are consistent with this notion: Norris and Randall showed that stimulation of the left and right ansae subclavia has a stronger inotropic effect in the RV than in the LV ${ }^{42}$. Similarly, Abe et al. ${ }^{43}$ reported stronger effects of sympathetic nerve stimulation on RV systolic pressure, which were not modified by a1-AR blockade. Thus, previous data suggested the existence of differential $\beta A R$ stimulation in RV versus LV, but until now its clear demonstration has been lacking and the potential mechanisms involved remain elusive. Our experiments show that ISO infusion in vivo exerts a stronger relative increase in RV contractility compared to that of the LV (Figure 1). Importantly, myocytes isolated from the midmyocardial layer of the $\mathrm{RV}$ showed enhanced and prolonged stimulation of $\mathrm{Ca}^{2+}$ transients and contraction by ISO compared to their LV counterparts (Figure 2). These results demonstrate that the high sensitivity of the RV to $\beta A R$ stimulation relies at least partly on an intrinsic property of RV myocytes, and raise the question of which mechanisms are involved.

BAR control of cardiac ECC operates mainly through activation of CAMP and PKA. Consistent with increased functional effects of $\beta A R s$, cytoplasmic cAMP and PKA 
activity were found increased in RVMs upon ISO pulse stimulation (Figure 3). However, this difference was compartment-specific since enhanced $\beta A R$ responses were not observed at the plasma membrane. Indeed neither $\beta A R$ regulation of subsarcolemmal CAMP signals nor that of $\mathrm{I}_{\mathrm{CaL}}$ and $\mathrm{I}_{\mathrm{Ks}}$ were modified in $\mathrm{RV}$ compared to LV (Figure 4). Since $\mathrm{I}_{\mathrm{CaL}}$ was not involved in the enhanced inotropic effect of ISO in RV, other ECC proteins must be differentially regulated. Likely candidates are RyR2 and PLB, which control SR $\mathrm{Ca}^{2+}$ release and reuptake by SERCA2a, respectively.

Although differential SERCA2a-PLB association was observed in RV versus LV in rat ${ }^{39}$, we did not observe differences in the decay kinetics of $\mathrm{Ca}^{2+}$ transients in RV versus LV dog myocytes (data not shown). To our knowledge, whether RyR2 and PLB are differentially phosphorylated in response to ISO stimulation in RV versus LV has not been investigated.

Enhanced $\beta A R$ functional effects in RVMs could result from increased cAMP generation in RVMs. However, the lack of difference at the plasma membrane (Figure 4) does not support this idea. Moreover, previous studies in dog indicate similar densities of $\beta A R s, A C$ activity and AC stimulation by $\beta$-ARs in RV versus LV $44-46$. Because in rodents PDEs can generate CAMP gradients within cardiac myocytes ${ }^{8}, 9,53,54$, we tested the hypothesis that they could be involved in the interventricular differences in cytoplasmic CAMP accumulation in dog. Our results indicate that PDE3 inhibition strongly increased maximal [CAMP]cyt in LVMs upon BAR stimulation, while having a weak effect on maximal [CAMP]cyt in RVMs, thus abolishing the differences between RVMs and LVMs. PDE3 inhibition also significantly prolonged the effect of ISO on [CAMP]pm in LVMs but not in RVMs (Figure 7A). PDE4 inhibition potentiated the maximal [CAMP]cyt in both RVMs and LVMs upon BAR stimulation, with a more pronounced effect in RVMs. As a consequence, the interventricular difference in [CAMP]cyt accumulation was attenuated upon PDE4 inhibition. We conclude that regionally-specific differences in subcellular cAMP compartmentation arise from a more stringent control of $\beta A R s$ CAMP signals by PDE3 and PDE4 in LVMs than in RVMs.

Upon $\beta A R$ stimulation, the relatively balanced contribution of PDE3 and PDE4 to CAMP hydrolysis in the cytoplasm (Figure 5) and the dominance of PDE3 at the sarcolemma (Figure 6) is at variance with previous results in rodents, where PDE4 is dominant in both compartments ${ }^{6-9}$. Because in dog PDE3 inhibitors have strong inotropic effects in contrast to PDE4 inhibitors ${ }^{18}$, our results raise the question of the functional role of PDE4 in dog. When the respective role of these two PDEs was evaluated on the stimulation of $\mathrm{I}_{\mathrm{CaL}}$ (Figure 7) by ISO, inhibition of PDE3 but not PDE4 significantly prolonged $\mathrm{I}_{\mathrm{CaL}}$ upregulation. This is consistent with a predominance of PDE3 in T-tubular membranes, whereas only a fraction of PDE4 is sarcolemmal and most of the activity is soluble ${ }^{18,19}$. However, when PDE3 was inhibited, PDE4 inhibition drastically prolonged $I_{\text {CaL }}$ recovery from $\beta A R$ stimulation 
(Figure 7). Thus, PDE4 can regulate $\mathrm{I}_{\mathrm{CaL}}$, but under normal conditions it is masked by the predominant PDE3. Therefore, the hierarchy between PDE3 and PDE4 appears to be opposite in dog compared to rat ${ }^{9}$.

In conclusion, the current study demonstrates that RV and LV differ in their sensitivity to $\beta A R$ stimulation both in vivo and in vitro, and reveals regionally-specific differences in $\beta A R$ coupling to PDE3 and PDE4 and subcellular CAMP compartmentation in the heart. The fact that these results were obtained in a large mammal increases the likelihood that they represent the human situation. If this is the case, such enhanced sensitivity to $\beta A R$ stimulation may be an important factor for RV dysfunction not only in the context of sports medicine, but also in RV failure consequent to left-sided heart failure or pulmonary hypertension.

\subsection{Acknowledgments}

We thank Florence Lefebvre for superb technical assistance, Dr. Viacheslav Nikolaev for providing the Epac2-camps and pmEpac2-camps, Dr. Jin Zhang for providing the AKAR3 sensor and Dr. Bertrand Crozatier for helpful discussions. 


\section{References}

(1) Bers DM. Cardiac excitation-contraction coupling. Nature. 2002;415:198-205.

(2) Marx SO, Kurokawa J, Reiken S, et al. Requirement of a macromolecular signaling complex for $\beta$ adrenergic receptor modulation of the KCNQ1-KCNE1 potassium channel. Science. 2002;295:496-499.

(3) Mika D, Leroy J, Vandecasteele G, Fischmeister R. PDEs create local domains of cAMP signaling. J Mol Cell Cardiol. 2012;52:323-329.

(4) Rochais F, Abi-Gerges A, Horner $K$, et al. A specific pattern of phosphodiesterases controls the CAMP signals generated by different $\mathrm{G}$ scoupled receptors in adult rat ventricular myocytes. Circ Res. 2006;98:1081-1088.

(5) Richter W, Xie M, Scheitrum C, et al. Conserved expression and functions of PDE4 in rodent and human heart. Basic Res Cardiol. 2011;106:249-62.

(6) Mongillo M, McSorley T, Evellin S, et al. Fluorescence resonance energy transfer-based analysis of CAMP dynamics in live neonatal rat cardiac myocytes reveals distinct functions of compartmentalized phosphodiesterases. Circ Res. 2004;95:65-75.

(7) Rochais F, Vandecasteele G, Lefebvre F, et al. Negative feedback exerted by PKA and CAMP phosphodiesterase on subsarcolemmal CAMP signals in intact cardiac myocytes. An in vivo study using adenovirus-mediated expression of CNG channels. J Biol Chem. 2004;279:52095-52105.

(8) Nikolaev VO, Bunemann M, Schmitteckert E, Lohse MJ, Engelhardt S. Cyclic AMP imaging in adult cardiac myocytes reveals far-reaching B1-adrenergic but locally confined B2-adrenergic receptor-mediated signaling. Circ Res. 2006;99:1084-1091.

(9) Leroy J, Abi-Gerges A, Nikolaev VO, et al. Spatiotemporal dynamics of Badrenergic cAMP signals and L-type $\mathrm{Ca}^{2+}$ channel regulation in adult rat ventricular myocytes: Role of phosphodiesterases. Circ Res. 2008;102:1091-1100.

(10) Baillie GS, Sood A, McPhee I, et al. $\beta$-Arrestin-mediated PDE4 cAMP phosphodiesterase recruitment regulates $\beta$-adrenoceptor switching from $G$ s to Gi. Proc Natl Acad Sci USA. 2003;100:941-945. 
(11) Richter W, Day P, Agraval R, et al. Signaling from B1- and B2 -adrenergic receptors is defined by differential interactions with PDE4. Embo J. 2008;27:384-393.

(12) De Arcangelis V, Liu R, Soto D, Xiang Y. Differential association of phosphodiesterase 4D isoforms with 32 -adrenoceptor in cardiac myocytes. J Biol Chem. 2009;284:33824-32.

(13) Lehnart SE, Wehrens XHT, Reiken S, et al. Phosphodiesterase 4D deficiency in the ryanodine receptor complex promotes heart failure and arrhythmias. Cell. 2005;123:23-35.

(14) Kerfant BG, Zhao D, Lorenzen-Schmidt I, et al. PI3Ky is required for PDE4, not PDE3, activity in subcellular microdomains containing the sarcoplasmic reticular calcium ATPase in cardiomyocytes. Circ Res. 2007;101:400-8.

(15) Beca S, Helli PB, Simpson JA, et al. Phosphodiesterase 4D regulates baseline sarcoplasmic reticulum $\mathrm{Ca}^{2+}$ release and cardiac contractility, independently of L-type $\mathrm{Ca}^{2+}$ current. Circ Res. 2011;109:1024-30.

(16) Leroy J, Richter W, Mika D, et al. Phosphodiesterase 4B in the cardiac L-type $\mathrm{Ca}^{2+}$ channel complex regulates $\mathrm{Ca}^{2+}$ current and protects against ventricular arrhythmias. J Clin Invest. 2011;121:2651-61.

(17) Terrenoire C, Houslay MD, Baillie GS, Kass RS. The cardiac $\mathrm{I}_{\mathrm{Ks}}$ potassium channel macromolecular complex includes the phosphodiesterase PDE4D3. J Biol Chem. 2009;284:9140-6.

(18) Weishaar RE, Kobylarz-Singer DC, Steffen RP, Kaplan HR. Subclasses of cyclic AMP-specific phosphodiesterase in left ventricular muscle and their involvement in regulating myocardial contractility. Circ Res. 1987;61:539-547.

(19) Lugnier C, Muller B, Lebec A, Beaudry C, Rousseau E. Characterization of indolidan-sensitive and rolipram-sensitive cyclic nucleotide phosphodiesterases in canine and human cardiac microsomal fractions. J Pharmacol Exp Ther. 1993;265:1142-1151.

(20) Smith C], Huang R, Sun D, et al. Development of decompensated dilated cardiomyopathy is associated with decreased gene expression and activity of the milrinone-sensitive CAMP phosphodiesterase PDE3A. Circulation. 1997;96:3116-3123. 
(21) Osadchii OE. Myocardial phosphodiesterases and regulation of cardiac contractility in health and cardiac disease. Cardiovasc Drugs Ther. 2007;21:171-94.

(22) Monrad ES, Baim DS, Smith HS, et al. Assessment of long-term therapy with milrinone and the effects of milrinone withdrawal. Circulation. 1986;73:III205-12.

(23) Molina CE, Leroy J, Xie M, et al. Cyclic AMP phosphodiesterase type 4 protects against atrial arrhythmias. J Am Coll Cardiol. 2012;59:2182-2190.

(24) Zaffran S, Kelly RG, Meilhac SM, Buckingham ME, Brown NA. Right ventricular myocardium derives from the anterior heart field. Circ Res. 2004;95:261-8.

(25) Verzi MP, McCulley DJ, De Val S, Dodou E, Black BL. The right ventricle, outflow tract, and ventricular septum comprise a restricted expression domain within the secondary/anterior heart field. Dev Biol. 2005;287:134-45.

(26) Ho SY, Nihoyannopoulos P. Anatomy, echocardiography, and normal right ventricular dimensions. Heart. 2006;92 Suppl 1:i2-13.

(27) Haddad F, Hunt SA, Rosenthal DN, Murphy DJ. Right ventricular function in cardiovascular disease, part I: Anatomy, physiology, aging, and functional assessment of the right ventricle. Circulation. 2008;117:1436-48.

(28) Sicouri S, Antzelevitch C. A subpopulation of cells with unique electrophysiological properties in the deep subepicardium of the canine ventricle. The M cell. Circ Res. 1991;68:1729-41.

(29) Antzelevitch C, Sicouri S, Litovsky SH, et al. Heterogeneity within the ventricular wall - Electrophysiology and pharmacology of epicardial, endocardial, and M-Cells. Circ Res. 1991;69:1427-1449.

(30) Zygmunt AC, Goodrow RJ, Antzelevitch C. $\mathrm{I}_{\mathrm{NaCa}}$ contributes to electrical heterogeneity within the canine ventricle. Am J Physiol Heart Circ Physiol. 2000;278:H1671-H1678.

(31) Gaborit N, Le Bouter S, Szuts V, et al. Regional and tissue specific transcript signatures of ion channel genes in the non-diseased human heart. $J$ Physiol. 2007;582:675-93. 
(32) Watanabe T, Delbridge LM, Bustamante JO, McDonald TF. Heterogeneity of the action potential in isolated rat ventricular myocytes and tissue. Circ Res. $1983 ; 52: 280-290$.

(33) Volders PG, Sipido KR, Carmeliet E, et al. Repolarizing $\mathrm{K}^{+}$currents $\mathrm{I}_{\mathrm{TO} 1}$ and $\mathrm{I}_{\mathrm{Ks}}$ are larger in right than left canine ventricular midmyocardium. Circulation. 1999;99:206-210.

(34) Di Diego JM, Sun ZQ, Antzelevitch C. I To and action potential notch are smaller in left vs. right canine ventricular epicardium. Am $\mathrm{J}$ Physiol. 1996;271:H548-61.

(35) Ramakers C, Vos MA, Doevendans PA, et al. Coordinated down-regulation of KCNQ1 and KCNE1 expression contributes to reduction of $\mathrm{I}_{\mathrm{Ks}}$ in canine hypertrophied hearts. Cardiovasc Res. 2003;57:486-496.

(36) Pandit SV, Kaur K, Zlochiver S, et al. Left-to-right ventricular differences in $\mathrm{I}_{\text {KATP }}$ underlie epicardial repolarization gradient during global ischemia. Heart Rhythm. 2011;8:1732-9.

(37) Choi SW, Ahn JS, Kim HK, et al. Increased expression of ATP-sensitive $\mathrm{K}^{+}$ channels improves the right ventricular tolerance to hypoxia in rabbit hearts. Korean J Physiol Pharmacol. 2011;15:189-94.

(38) Phillips D, Aponte AM, Covian R, et al. Homogenous protein programming in the mammalian left and right ventricle free walls. Physiol Genomics. 2011;43:1198-206.

(39) Sathish V, Xu A, Karmazyn M, Sims SM, Narayanan N. Mechanistic basis of differences in $\mathrm{Ca}^{2+}$-handling properties of sarcoplasmic reticulum in right and left ventricles of normal rat myocardium. Am J Physiol Heart Circ Physiol. 2006;291:H88-96.

(40) Wang GY, McCloskey DT, Turcato S, et al. Contrasting inotropic responses to a1-adrenergic receptor stimulation in left versus right ventricular myocardium. Am J Physiol Heart Circ Physiol. 2006;291:H2013-7.

(41) La Gerche A, Heidbuchel H, Burns AT, et al. Disproportionate exercise load and remodeling of the athlete's right ventricle. Med Sci Sports Exerc. 2011;43:974-81.

(42) Norris JE, Randall WC. Responses of the canine myocardium to stimulation of thoracic cardiac nerves. Am J Physiol. 1977;232:H485-94. 
(43) Abe $Y$, Saito $D$, Tani $H$, et al. The effect of cardiac sympathetic nerve stimulation on the right ventricle in canine heart. Jpn Circ J. 1987;51:535-42.

(44) Vatner DE, Homcy CJ, Sit SP, Manders WT, Vatner SF. Effects of pressure overload, left ventricular hypertrophy on B-adrenergic receptors, and responsiveness to catecholamines. J Clin Invest. 1984;73:1473-82.

(45) Calderone A, Bouvier M, Li K, et al. Dysfunction of the B- and $\alpha$-adrenergic systems in a model of congestive heart failure. The pacing-overdrive dog. Circ Res. 1991;69:332-43.

(46) White $M$, Roden $R$, Minobe $W$, et al. Age-related changes in B-adrenergic neuroeffector systems in the human heart. Circulation. 1994;90:1225-1238.

(47) Nikolaev VO, Bunemann M, Hein L, Hannawacker A, Lohse MJ. Novel single chain CAMP sensors for receptor-induced signal propagation. $J$ Biol Chem. 2004;279:37215-37218.

(48) Wachten S, Masada N, Ayling L, et al. Distinct pools of cAMP centre on different isoforms of adenylyl cyclase in pituitary-derived GH3B6 cells. J Cell Sci. 2010;123:95-106.

(49) Allen MD, Zhang J. Subcellular dynamics of protein kinase A activity visualized by FRET-based reporters. Biochem Biophys Res Commun. 2006;348:716-21.

(50) Verde I, Vandecasteele G, Lezoualc'h F, Fischmeister R. Characterization of the cyclic nucleotide phosphodiesterase subtypes involved in the regulation of the L-type $\mathrm{Ca}^{2+}$ current in rat ventricular myocytes. $\mathrm{Br} \mathrm{J}$ Pharmacol 1999;127:65-74.

(51) Sanguinetti MC, Jurkiewicz NK. Role of external $\mathrm{Ca}^{2+}$ and $\mathrm{K}^{+}$in gating of cardiac delayed rectifier $\mathrm{K}^{+}$currents. Pflügers Arch. 1992;420:180-186.

(52) La Gerche A, Burns AT, Mooney DJ, et al. Exercise-induced right ventricular dysfunction and structural remodelling in endurance athletes. Eur Heart J. 2012;33:998-1006.

(53) Jurevicius J , Fischmeister R. cAMP compartmentation is responsible for a local activation of cardiac $\mathrm{Ca}^{2+}$ channels by $\mathrm{B}$-adrenergic agonists. Proc Natl Acad Sci USA. 1996;93:295-299.

(54) Zaccolo M, Pozzan T. Discrete microdomains with high concentration of cAMP in stimulated rat neonatal cardiac myocytes. Science. 2002;295:1711-1715. 
Chapter 6

Reduced Ventricular Proarrhythmic Potential of the Novel Combined I on-Channel Blocker AZD1305 versus Dofetilide in Dogs With Remodeled Hearts

Circ Arrhythm Electrophysiol. 2012;5(1):201-9

Daniel M Johnson • Monique MJ de Jong • Harry JGM Crijns • Leif GA Carlsson •

Paul GA Volders 


\section{Abstract}

AZD1305 is an investigational antiarrhythmic agent for management of atrial fibrillation. It blocks various cardiac ion currents at different potencies and has atrialpredominant electrophysiological effects. We investigated the electrophysiological and proarrhythmic effects of AZD1305 versus dofetilide in dogs with chronic complete atrioventricular block (AVB) and myocardial hypertrophic remodeling. In the present study AZD1305 was administered to anesthetized mongrel dogs before and $>2$ weeks after the induction of AVB and ventricular and atrial electrophysiological parameters were assessed. In all dogs, the selective $\mathrm{I}_{\mathrm{Kr}}$ blocker dofetilide was used to examine susceptibility to acquired torsades de pointes (TdP) in chronic AVB and for comparison. At normal sinus rhythm, AZD1305 increased QT and RR intervals from $290 \pm 7$ to $397 \pm 15 \mathrm{~ms}(+37 \%, \mathrm{P}<0.0001)$ and from $603 \pm 22$ to $778 \pm 32 \mathrm{~ms}(+29 \%, \mathrm{P}=0.002)$, respectively. In the same animals at chronic AVB, AZD1305 increased the QT interval from $535 \pm 28$ to $747 \pm 36 \mathrm{~ms}(+40 \%, \mathrm{P}<0.05)$, similar to the QT prolongation by dofetilide ( $511 \pm 22$ to $703 \pm 45 \mathrm{~ms}(+38 \%$, $P<0.0001))$. AZD1305 slightly slowed the idioventricular rhythm. Whereas all $(n=14)$ chronic-AVB animals exhibited TdP upon dofetilide, the arrhythmia was induced in only 4/11 dogs after AZD1305. Beat-to-beat variability of left-ventricular (LV) monophasic-action-potential duration (MAPD90) increased after dofetilide (2.3 \pm 0.2 to $6.3 \pm 0.7 \mathrm{~ms} ; \mathrm{P}<0.00010)$, but not after AZD1305 (2.8 \pm 0.3 to $3.7 \pm 0.3 \mathrm{~ms}$; $\mathrm{P}=0.20$ ), despite similar LV MAPD90 prolongations. Despite causing similar degrees of repolarization delay as the selective $\mathrm{I}_{\mathrm{Kr}}$ blocker dofetilide, the combined ionchannel blocker AZD1305 induces less repolarization instability and has a lower ventricular proarrhythmic potential in the remodeled dog heart. 


\subsection{I ntroduction}

Atrial fibrillation (AF) is the most common cardiac arrhythmia in the Western world, with age-related prevalence reaching $>10 \%$ in octogenarians ${ }^{1}$. It has been predicted that over 5.6 million patients in North-America will be diagnosed with AF by 2050 ? This arrhythmia is a frequent complication of uncorrected hypertension, myocardial ischemia or valvular disease. Moreover, familial AF or lone AF without apparent familial segregation is increasingly recognized.

Currently, the pharmacological treatments aimed at maintaining sinus rhythm include class I, II and III antiarrhythmic agents. Termination of persistent AF may require any of these options (with variable effectiveness) or electrical cardioversion. However, none of the aforementioned therapies are considered atrial-specific and cause effects on ventricular electrical activation and repolarization, and some carry an inherent risk of ventricular proarrhythmia.

The proarrhythmic liability of anti-AF drugs could potentially be circumvented by designing compounds with composite actions on multiple ion channels. Vernakalant, a predominantly atrial-selective compound targeting $\mathrm{I}_{\mathrm{Kur}}, \mathrm{I}_{\mathrm{Kr}}, \mathrm{I}_{\mathrm{TO}}$ and $\mathrm{I}_{\mathrm{Na}}{ }^{3}$ was recently approved in Europe for the rapid conversion of recent (seven days or less) onset AF to sinus rhythm. Clinically, vernakalant has been shown to be effective in restoring sinus rhythm in patients with AF episodes lasting between 3 hours and 7 days ${ }^{4}$ with a low proarrhythmic risk. This compound has been shown to be less efficacious in patients with congestive heart failure ${ }^{5}$. However to date, no results have been reported on patients with severe heart failure in whom cardiac remodeling could predispose to altered drug efficacy or adverse side effects. Dronedarone is another new agent for the management of AF with composite actions at multiple ion channels. Although this compound may have an improved safety profile compared to amiodarone, it is less efficacious in reducing $\mathrm{AF}$ recurrence rates ${ }^{6}$.

AZD1305 (Figure 1A) is an investigational compound that has been under development for the management of persistent AF. Although AZD1305 predominantly blocks $I_{\mathrm{kr}}$ it also has major inhibitory effects on $I_{\mathrm{CaL}}$ and $\mathrm{I}_{\mathrm{Na}}$ (predominantly $\mathrm{I}_{\text {NaLate }}$ ) and minor effects on other $\mathrm{K}^{+}$currents ${ }^{7,8}$. In the normal canine heart, this compound exerts atrial-predominant electrophysiological effects, both in vivo and in vitro ${ }^{9}$. In the anesthetized methoxamine-sensitized rabbit model of torsades de pointes ventricular tachyarrhythmia (TdP), intravenous AZD1305 increased the QT interval without inducing ventricular extrasystoles or TdP ${ }^{7}$. Beat-tobeat variability of repolarization duration (BVR of the QT interval) also remained unaltered. In contrast, the selective $\mathrm{I}_{\mathrm{Kr}}$ blocker dofetilide prolonged the QT interval, increased BVR and induced TdP in the majority of animals tested ${ }^{7}$. AZD1305 has been shown to depress excitability and suppress delayed-afterdepolarization-induced triggered activity in canine pulmonary-vein sleeve preparations ${ }^{10}$. Recent clinical 
data suggest that this compound has electrophysiological effects that may translate into antiarrhythmic efficacy in AF patients and may have a reduced proarrhythmic potential as compared to selective $\mathrm{I}_{\mathrm{Kr}}$ blockers ${ }^{11,12}$.

In the present study, we investigated the electrophysiological effects of AZD1305 in anesthetized dogs with remodeled hearts, with our hypothesis being that due to the composite ion-channel blockade by AZD1305, less ventricular proarrhythmia will be seen when compared to $I_{\mathrm{Kr}}$ blockade alone, despite leading to equivalent repolarization prolongation. To this aim, we used the dog with chronic complete atrioventricular block (AVB), a sensitive model to examine the proarrhythmic potential of new chemical entities. After the induction of AVB, the heart undergoes electrical and structural remodeling, creating a substrate for $\operatorname{TdP}^{13}{ }^{14}$. We analyzed ventricular and atrial repolarization parameters and compared the effects of AZD1305 with those of the selective $I_{\mathrm{Kr}}$ blocker dofetilide (Figure 1A), which exerts torsadogenic actions in this animal model.

\subsection{Materials and Methods}

\subsubsection{General}

Animal handling was in accordance with the Dutch Law on Animal Experimentation and the European Directive for the Protection of Vertebrate Animals Used for Experimental and Other Scientific Purposes, European Community Directive 86/609/CEE, and under the regulations of the Committee for Experiments on Animals of Maastricht University, The Netherlands.

Fourteen adult mongrel dogs (10 female and 4 male; body weight $20.1 \pm 0.8 \mathrm{~kg}$; Marshall BioResources, NY, USA) were included in these experiments. After overnight fasting, animals were sedated with $0.5 \mathrm{mg} / \mathrm{kg}$ methadone, $0.5 \mathrm{mg} / \mathrm{kg}$ acepromazine, and $0.5 \mathrm{mg}$ atropine intramuscularly. Anesthesia was induced with sodium pentobarbital $25 \mathrm{mg} / \mathrm{kg}$ intravenously and maintained by isoflurane ( $0.5 \%$ to $1 \%)$. After intubation, the dogs were artificially ventilated with a mixture of oxygen and compressed air ( $2: 1)$. Proper animal care was taken before, during, and after the experiments, including a thermal mattress to maintain body temperature, fluid administration to prevent volume depletion $(0.9 \% \mathrm{NaCl})$, and administration of antibiotics (ampicillin $1000 \mathrm{mg}$ intramuscularly) and analgesics (buprenorphine 0.015 $\mathrm{mg} / \mathrm{kg}$ intramuscularly).

In all experiments, standard lead and precordial ECG registrations were combined with endocardial monophasic action potential (MAP) recordings and continuously stored. MAPs (Hugo Sachs Elektronik, Germany) were recorded from left ventricular $(\mathrm{LV})$, right ventricular (RV) and right atrial (RA) endocardial sites. MAP signals were 
accepted on the basis of amplitude, morphology and stability. MAP catheters were kept in the same position during drug infusions.

\subsubsection{Experimental Design}

The experimental protocol is shown in Figure 1B. AZD1305 was dissolved in an equimolar amount of $0.1 \mathrm{~mol} / \mathrm{L}$ tartaric acid and then diluted in $0.9 \%$ saline. AZD1305 was infused at a rate of $36 \mu \mathrm{g} / \mathrm{kg} / \mathrm{min}$ for a total of $30 \mathrm{~min}>1$ week before creating $A V B$ to investigate the effects of this compound in the normal unremodeled heart with sinus rhythm, and in acute and chronic AVB. AVB was induced by radiofrequency catheter ablation of the His bundle as previously described ${ }^{15}$.

Dofetilide was dissolved in $0.9 \%$ saline and intravenously administered at a dose of $12.5 \mu \mathrm{g} / \mathrm{kg} / 5 \mathrm{~min}$ to test for TdP inducibility 2-3 weeks after inducing AVB (chronic AVB) in all dogs. Three animals developed intractable ventricular fibrillation during TdP. To investigate whether AZD1305 suppresses dofetilide-induced TdP, a bolus $(870 \mu \mathrm{g} / \mathrm{kg})$ of the compound was administered after the first episode of TdP in 4 dogs (Figure 1B). In 4 animals $12.5 \mu \mathrm{g} / \mathrm{kg} / 5$ min dofetilide was also infused $>1$ week before AVB was created so a comparison of atrial effects of this compound could be made pre and post remodeling, and compared with AZD1305.

In a total of 11 chronic-AVB dogs, AZD1305 (36 $\mu \mathrm{g} / \mathrm{kg} / \mathrm{min}$ for $30 \mathrm{~min}$ ) was administered at $3 \pm 1$ weeks after the induction of AVB (chronic AVB) to examine its electropharmacological effects in the remodeled heart. In 4 of these experiments, after the AZD1305 infusion, a dofetilide challenge was reapplied to investigate preventive effects of AZD1305 against dofetilide-induced TdP (Figure 1B).

When TdP did not stop spontaneously within 10 to 20 seconds or when the arrhythmia deteriorated into ventricular fibrillation, the dog was electrically cardioverted. If TdP recurred in a period longer than 10 minutes magnesium sulphate (100 mg/kg for $2 \mathrm{~min}$ ) was used to restore a regular rhythm.

After sacrifice, hearts were excised and weighed ( $n=11$ dogs with chronic AVB). Average heart weights were $217 \pm 11 \mathrm{~g}$. Heart-weight to body-weight ratios averaged $10.5 \pm 0.2 \mathrm{~g} / \mathrm{kg}$, similar to previous studies on chronic AVB and significantly higher than unremodeled dog hearts ${ }^{13,14,15}$. 
A

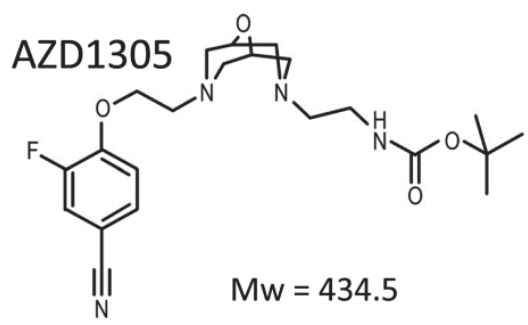<smiles>CN(CCOc1ccc(NS(C)(=O)=O)cc1)CCc1ccc(NS(C)(=O)=O)cc1</smiles>

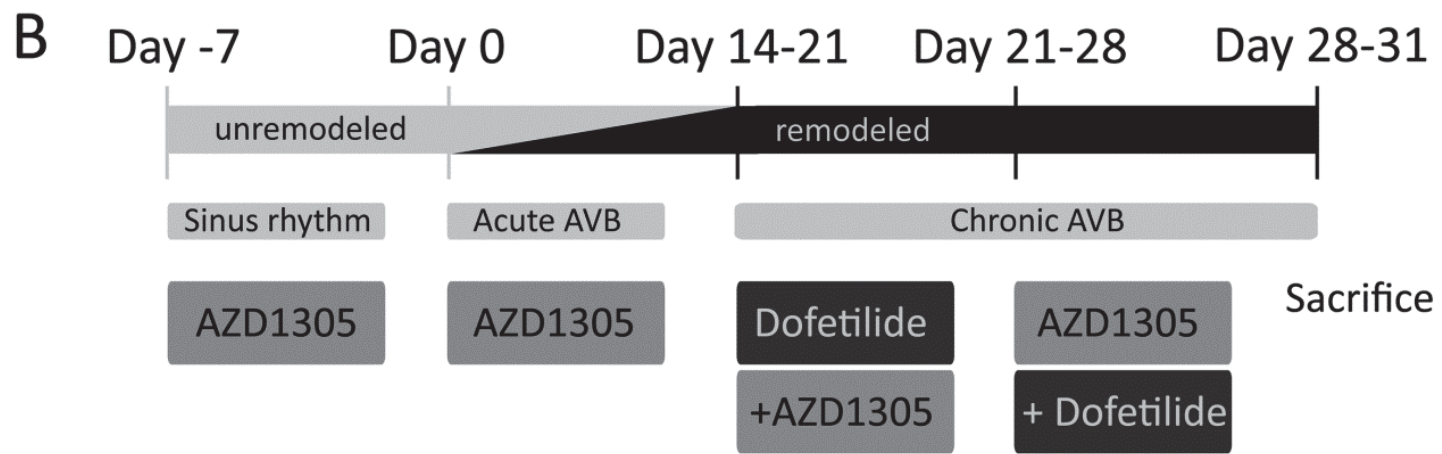

Figure 1: A. Chemical structures of the combined ion-channel blocker AZD1305 and the pure $\mathrm{I}_{\mathrm{kr}}$ blocker dofetilide. B. Experimental protocol.

\subsubsection{Plasma Analysis of AZD1305}

Venous blood samples were obtained at baseline and then at 5, 10, 15, 30, 35, 45, 60, 90, 120 and 150 min after start of AZD1305 infusion. Samples were collected in K2-EDTA tubes, centrifuged for $10 \mathrm{~min}$ at $4000 \mathrm{rpm}$ at $4^{\circ} \mathrm{C}$ and the plasma stored at $-80^{\circ} \mathrm{C}$. The plasma concentration of AZD1305 was determined by on-line solid-phase extraction followed by tandem mass spectrometric detection. The lower limit of quantification was $0.0150 \mu \mathrm{mol} / \mathrm{L}$.

\subsubsection{Data Analysis}

RR, QRS, QT, PP and PR intervals in ECG lead II, LV and RV MAP duration (LV and RV MAPD) at 90\% repolarization (MAPD90), and RA MAPD at 50\% (MAPD50) and $90 \%$ repolarization were measured offline using a custom-made computer program (IDEEQ, IDEE, Maastricht University). QT intervals were corrected for heart rate changes according to Van de Water et al. (QTcV=QT-0.087(RR-1000) ${ }^{16}$. Most data were averaged from 30 consecutive beats measured at -5 and 0 min (baseline) and at $5,10,15,30,35,45$ and 60 min after start of AZD1305 or dofetilide infusion. If extrasystolic activity was seen, parameters were measured before the first extrasystole. Beat-to-beat variability of repolarization duration (BVR) was quantified from LV MAPD90 of 30 consecutive beats as follows: BVR $=\Sigma\left(\right.$ LV MAPD $\left._{n}-L_{\text {MAPDn-1 }}\right)$ / (30* $\sqrt{2})$. 
Electrophysiological parameters were compared using (repeated-measures) ANOVA followed by Bonferroni's test. Data are reported as mean \pm SEM. Countables, such as TdP episode numbers, were tested with a Mann-Whitney rank sum test and are reported as median (interquartile range). Differences were considered statistically significant if $\mathrm{P}<0.05$.

\subsection{Results}

\subsubsection{Concentration-Dependent Prolongation of Ventricular Repolarization by AZD1305 in the Normal Dog Heart}

In 14 normal anesthetized dogs, intravenous administration of AZD1305 resulted in a maximal plasma concentration of $1.57 \pm 0.05 \mu \mathrm{mol} / \mathrm{L}$ at $30 \mathrm{~min}$ (Figure 2). The QT and QTcV interval increased from $290 \pm 7 \mathrm{~ms}$ and $324 \pm 6 \mathrm{~ms}$ at baseline to $397 \pm 15$ ms and $417 \pm 14 \mathrm{~ms}$ at $30 \mathrm{~min}$ AZD1305, respectively (+37\% and $+29 \%, \mathrm{P}<0.0001$ ), along with an increase in the RR interval (Figure 2 and Table 1). Repolarization prolonged in a concentration-dependent manner, with MAPD90 increasing more in the LV than in the RV ( $+48 \%$ versus $+39 \%$; Figure 2 ). LV and RV BVR did not change significantly. P-wave duration and the QRS interval were slightly but significantly increased by AZD1305 (Table 1). The compound did not induce ventricular or atrial arrhythmias in these normal hearts. After stopping infusion, AZD1305 plasma levels rapidly declined, reaching $0.57 \pm 0.05 \mu \mathrm{mol} / \mathrm{L} 30 \mathrm{~min}$ later. 


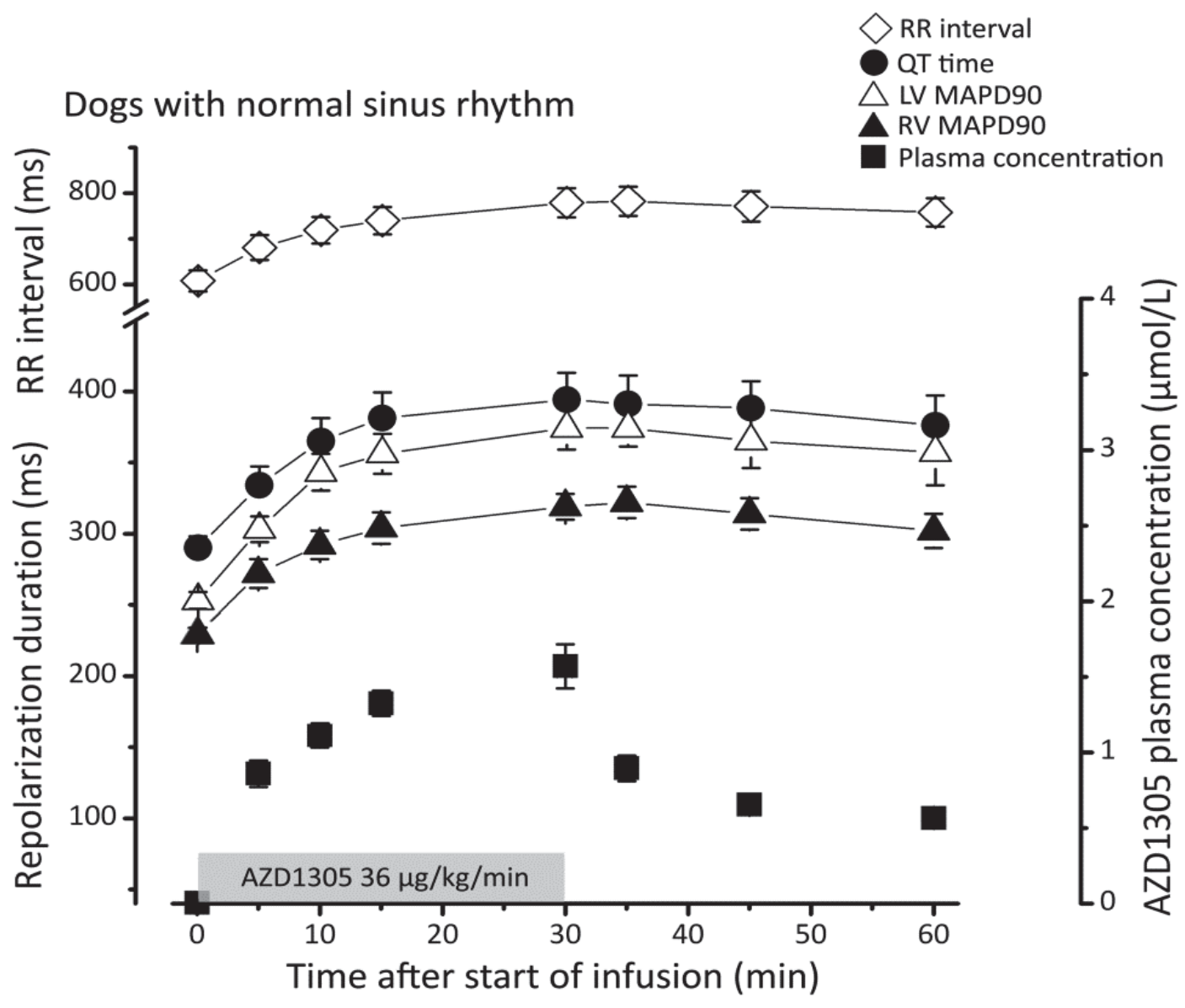

Figure 2: Rate-Dependent Repolarization Effects of AZD1305 in the Normal, AcutelyOverloaded and Chronically-Remodeled Dog Heart. Changes in QT interval, LV and RV MAPD90, and RR interval in relation to plasma concentration of AZD1305 $(36 \mu \mathrm{g} / \mathrm{kg} / \mathrm{min}$ intravenously for $30 \mathrm{~min}$ ) in the normal canine heart with sinus rhythm.

In 6 dogs, steady-state pacing was applied at a cycle length (CL) of 500 ms in the normal heart (right-atrial pacing), and at $500 \mathrm{~ms}$ and $1000 \mathrm{~ms}$ just after AVB induction and at chronic AVB (RV-apex pacing). Figure 3 illustrates the ratedependent effects of AZD1305 (versus baseline) on LV MAPD90. Effects during pacing are plotted next to those during sinus or idioventricular rhythm. At a pacing $\mathrm{CL}$ of $500 \mathrm{~ms}$, AZD1305 led to a 20\% increase of LV MAPD90 in the normal heart, $30 \%$ increase at acute AVB and $37 \%$ prolongation at chronic AVB at 3 weeks. Reverse use-dependent prolongation of LV and RV MAPD90 was most accentuated in the remodeled heart at chronic AVB. 
Sinus rhythm

Acute AVB

Chronic AVB
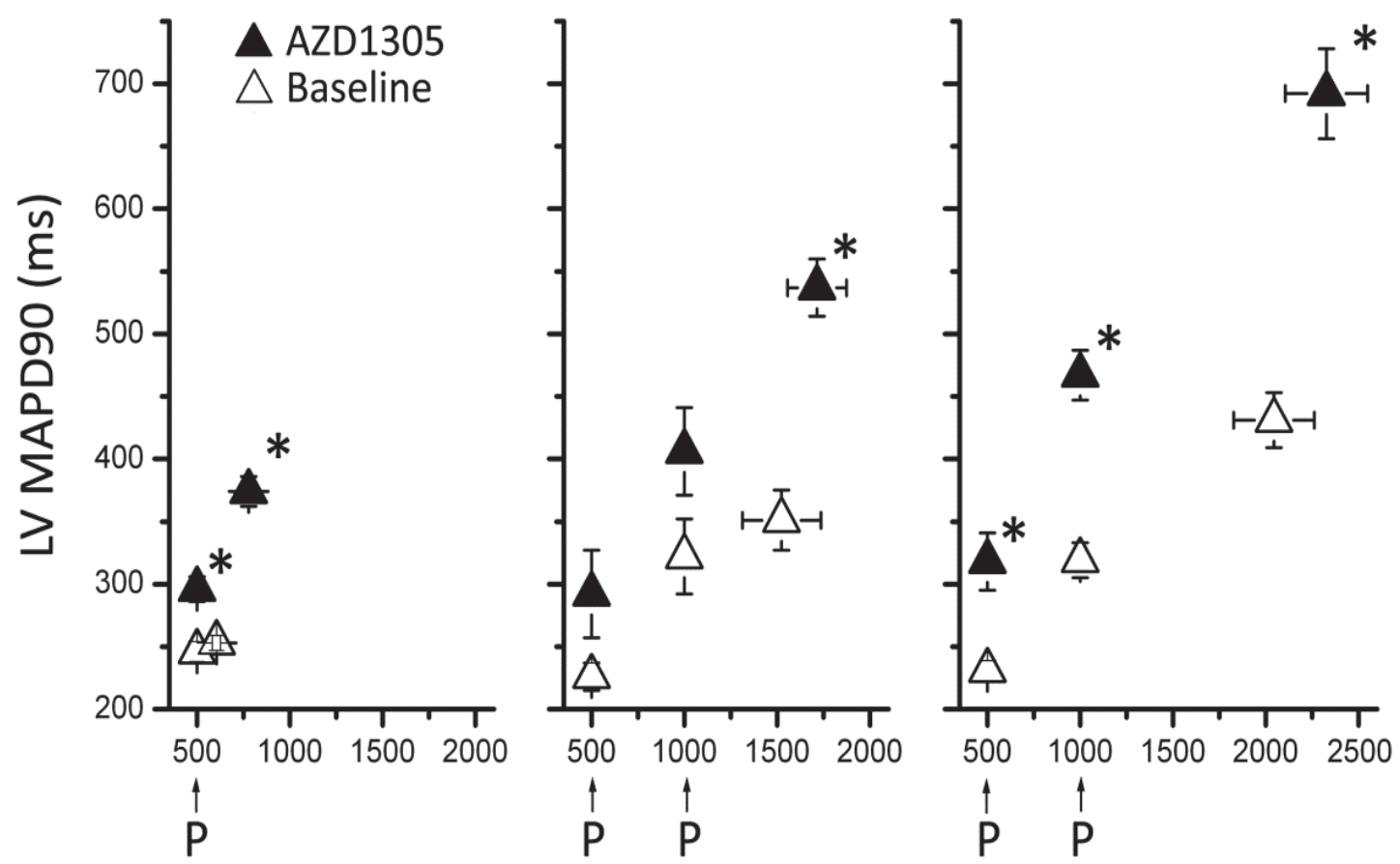

RR interval (ms)

Figure 3: Rate-dependent repolarization effects of AZD1305 at normal sinus rhythm, acute and chronic AVB. Depicted are the changes in LV MAPD90 before (open triangles) and after (closed triangles) treatment at different intrinsic (sinus/idioventricular) and paced rates ("P"; right-atrial pacing in normal heart; RV pacing in acute and chronic AVB). ${ }^{*}, \mathrm{P}<0.05$ versus before treatment at the same $\mathrm{CL}$ (or sinus/idioventricular rate).

\subsubsection{Repolarization Prolongation and Proarrhythmic Potential by AZD1305 and Dofetilide in the Chronic-AVB Dog Heart}

In chronic AVB, intravenous administration of AZD1305 resulted in a maximal plasma concentration of $1.77 \pm 0.29 \mu \mathrm{mol} / \mathrm{L}$ at $30 \mathrm{~min}$ (Figure 4) decreasing to $0.66 \pm 0.10$ $\mu \mathrm{mol} / \mathrm{L} 30 \mathrm{~min}$ after stop of infusion. Plasma concentrations at all time points were not significantly different compared to those seen in sinus rhythm. The QT and QTcV interval increased from $535 \pm 28 \mathrm{~ms}$ and $444 \pm 30 \mathrm{~ms}$ at baseline to $747 \pm 36 \mathrm{~ms}$ and $634 \pm 35 \mathrm{~ms}$ at $30 \mathrm{~min}$ AZD1305, respectively ( $+40 \%$ and $+43 \%, \mathrm{P}<0.0001$; Table 1). A slowing of the idioventricular rate was noted (Table 1). The LV MAPD90 was prolonged from $431 \pm 22 \mathrm{~ms}$ to $692 \pm 34 \mathrm{~ms}(+61 \%, \mathrm{P}<0.0001)$ whereas the LV BVR remained unaltered $(2.8 \pm 0.3$ versus $3.7 \pm 0.3 \mathrm{~ms}, \mathrm{P}=0.20)$. Similar results were obtained for the RV repolarization variables. Figure 5 illustrates changes in repolarization induced by AZD1305 infusion in the same dog both at normal sinus rhythm and chronic AVB. 
Dogs with chronic AVB

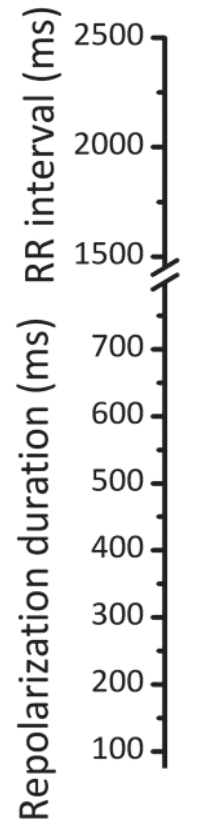

RR interval

QT time

$\triangle$ LV MAPD90

$\triangle$ RV MAPD90

Plasma concentration

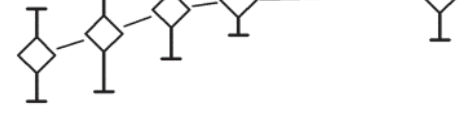

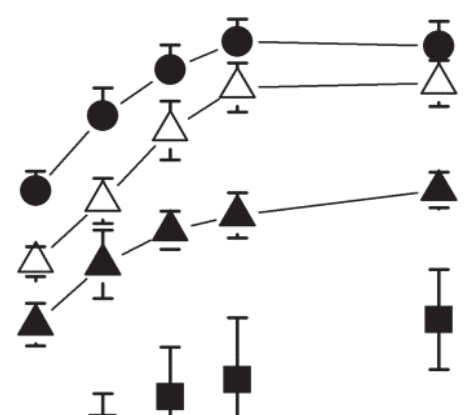

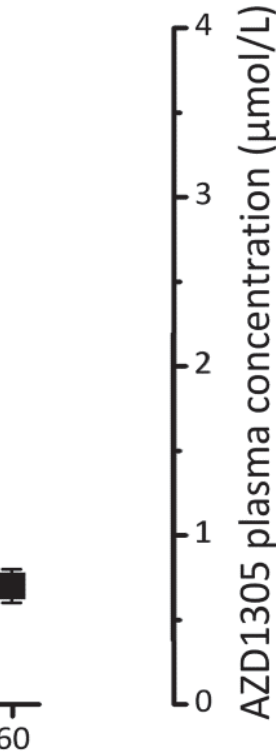

AZD1305 $36 \mu \mathrm{g} / \mathrm{kg} / \mathrm{min}$

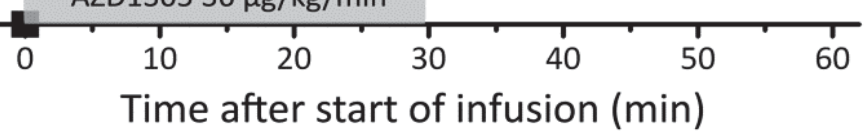

Figure 4: Changes in QT interval, LV and RV MAPD90, and RR interval in relation to plasma concentration of AZD1305 (36 $\mathrm{mg} / \mathrm{kg} / \mathrm{min}$ intravenously for $30 \mathrm{~min}$ ) in the remodeled canine heart with chronic AVB.

In separate experiments, dofetilide was administered to test for TdP inducibility. Similar to AZD1305, dofetilide prolonged the QT and QTCV interval, by $38 \%$ and $41 \%$, respectively (Table 1). The LV MAPD90 was prolonged by $51 \%$ ( $411 \pm 17 \mathrm{~ms}$ to $625 \pm 26 \mathrm{~ms} ; \mathrm{P}<0.0001)$ and was accompanied by an increase in BVR from $2.3 \pm 0.2$ $\mathrm{ms}$ to $6.3 \pm 0.7 \mathrm{~ms}(\mathrm{P}<0.0001)$. 
Sinus rhythm

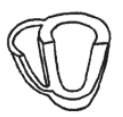

ECG II

LV

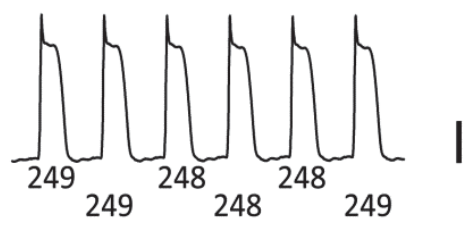

Chronic AVB

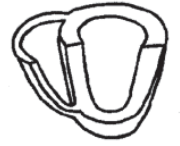

\section{Baseline}
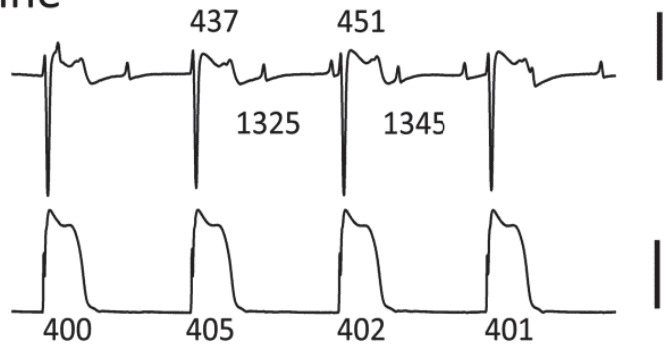

\section{AZD1305}

ECG II

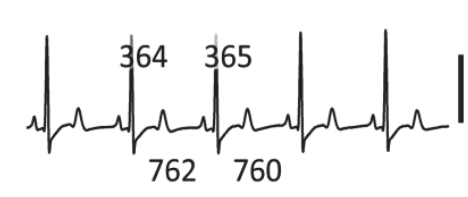

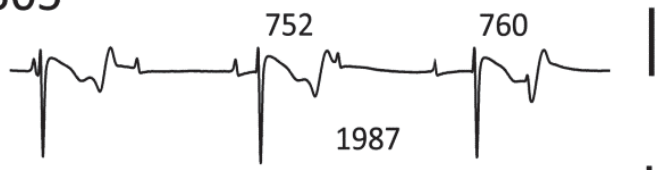
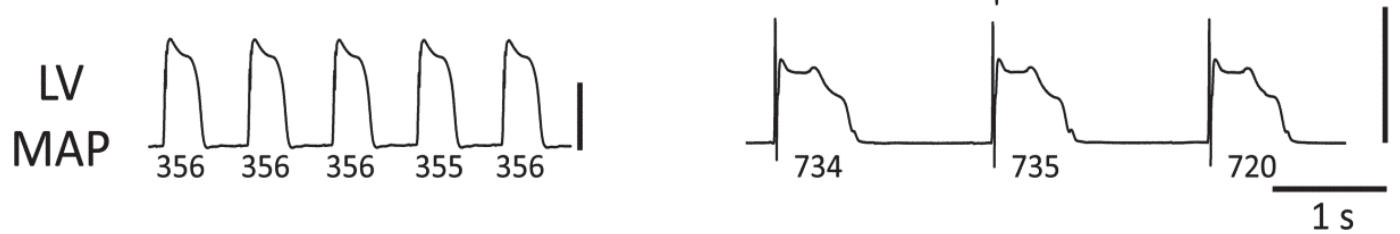

Figure 5: Effects of AZD1305 on ventricular repolarization in the same dog before and after cardiac remodeling by chronic AVB. Representative recordings of ECG lead II and LV MAPS before (Baseline) and at the end of the 30-min infusion of AZD1305. MAPD90 (ms) is indicated below the LV MAP signals. RR and QT intervals (ms) are shown below and above the ECGs, respectively. Vertical calibration bars depict 1 and $10 \mathrm{mV}$ for ECG and MAP recordings, respectively. 


\begin{tabular}{|lccc|ccccccc|}
\hline & Control & AZD 1305 & P & Control & Dofetilide & $P$ & Control & AZD 1305 & $P$ \\
\hline PP & $611 \pm 24$ & $780 \pm 34^{*}$ & $<0.0001$ & $591 \pm 18$ & $733 \pm 19 *$ & $<0.0001$ & $602 \pm 17$ & $760 \pm 33^{*}$ & $=0.0006$ \\
RR & $603 \pm 22$ & $778 \pm 32^{*}$ & $<0.0001$ & $1775 \pm 124$ & $1863 \pm 95$ & $=0.79$ & $2044 \pm 217$ & $2326 \pm 221^{*}$ & $=0.002$ \\
QT & $290 \pm 7$ & $397 \pm 15^{*}$ & $<0.0001$ & $511 \pm 22$ & $703 \pm 45^{*}$ & $=0.0009$ & $535 \pm 28$ & $747 \pm 36^{*}$ & $<0.0001$ \\
QTcV & $324 \pm 6$ & $417 \pm 14^{*}$ & $<0.0001$ & $441 \pm 17$ & $624 \pm 41^{*}$ & $<0.0001$ & $444 \pm 30$ & $634 \pm 35^{*}$ & $<0.0001$ \\
Pwave duration & $72 \pm 3$ & $77 \pm 3^{*}$ & $<0.0001$ & & & & & & \\
PR duration & $122 \pm 3$ & $125 \pm 3$ & $\mathrm{P}=0.20$ & & & & & & & \\
QRS duration & $75 \pm 5$ & $79 \pm 5 *$ & $=0.0007$ & & & & & & & \\
LVMAPD90 & $253 \pm 6$ & $374 \pm 15^{*}$ & $<0.0001$ & $411 \pm 17$ & $625 \pm 26 *$ & $<0.0001$ & $431 \pm 22$ & $692 \pm 34^{*}$ & $<0.0001$ \\
RVMAPD90 & $229 \pm 5$ & $319 \pm 9 *$ & $<0.0001$ & $332 \pm 34$ & $472 \pm 22^{*}$ & $=0.004$ & $339 \pm 31$ & $536 \pm 26 *$ & $=0.02$ \\
LVBVR & $0.6 \pm 0.1$ & $0.9 \pm 0.2$ & $=0.07$ & $2.3 \pm 0.2$ & $6.3 \pm 0.7 *$ & $<0.0001$ & $2.8 \pm 0.3$ & $3.7 \pm 0.3$ & $=0.20$ \\
RVBVR & $1.0 \pm 0.3$ & $1.1 \pm 0.3$ & $=0.69$ & $2.9 \pm 0.8$ & $5.6 \pm 1.7$ & $=0.18$ & $5.4 \pm 2.4$ & $5.6 \pm 1.9$ & $=0.98$ \\
TdPInducible & & & & & & & & & & \\
\end{tabular}

Table 1: Ventricular electrophysiological and proarrhythmic effects of AZD1305 and the reference compound dofetilide in the normal and remodeled canine heart. $*, \mathrm{P}<0.05$ versus baseline. SR indicates sinus rhythm; CAVB, chronic complete atrioventricular block; LV, left ventricular; MAPD, monophasic-action-potential duration; RV, right ventricular; BVR, beat-tobeat variability of repolarization; TdP, torsades de pointes.

Next, we examined the appearance of ventricular extrasystolic beats in the presence of AZD1305 and dofetilide. In the case of AZD1305, these were observed at 6.2 \pm 0.3 min after the start of infusion in 6/11 dogs. Except for two dogs, no ventricular extrasystolic beats occurred beyond $8 \pm 1$ min during the remainder of the experiment in any of the animals. By contrast, during exposure to dofetilide, ventricular extrasystoles were induced in all dogs ( $P=0.009$ versus $A Z D 1305)$, on average at $4.1 \pm 0.1 \mathrm{~min}$. There were no repolarization differences in beats prior, during and after extrasystolic activity (Figure 6). 
A

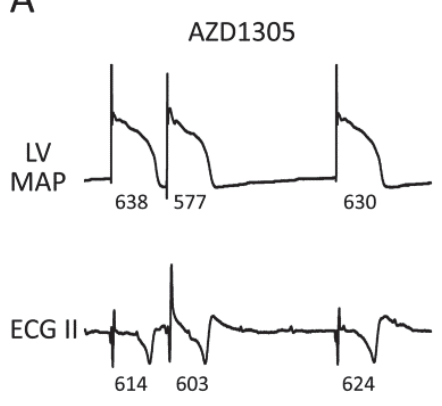

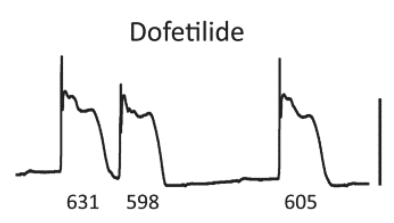

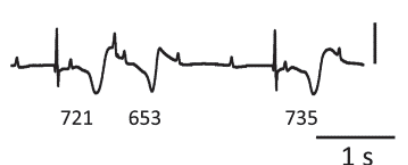

B

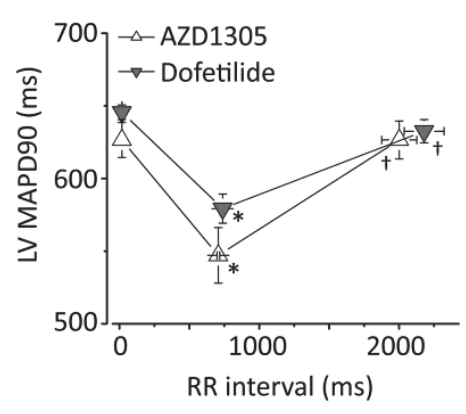

Figure 6: Repolarization duration of ventricular extrasystolic and post-extrasystolic beats after AZD1305 and dofetilide in the chronic-AVB dog. A. Representative examples of ECGs and LV MAPs. QT intervals and MAPD90 values (ms) are indicated below the traces. Vertical calibration bars depict 1 and $10 \mathrm{mV}$ for ECG and MAP recordings, respectively. B. Average values for LV MAPD90 as a function of RR CL for the last beat of the idioventricular rhythm, the ventricular extrasystole and the post-extrasystolic beat, respectively. Data are from all 4 dogs that exhibited ventricular extrasystoles during AZD1305. *, $\mathrm{P}<0.05$ versus baseline beat;,$+ \mathrm{P}<0.05$ versus extrasystole.

BVR was significantly different during infusion of AZD1305 versus dofetilide. Figure 7 illustrates how both LVMAPD90 and BVR were altered in two chronic AVB animals after either AZD1305 or dofetilide infusion. Dofetilide caused a progressive and parallel increase in LVMAPD90 and BVR promoting extrasystolic activity and TdP. In contrast, AZD1305 infusion did not increase BVR despite significantly prolonging MAPD90 (see also Table 1). Whereas dofetilide caused TdP in all dogs, this occurred in only 4 out of 11 dogs after AZD1305 ( $P=0.0007$ versus dofetilide). Furthermore, dofetilide-induced TdP episodes occurred more frequently, lasted longer and required electrical cardioversion more often. The cycle lengths of AZD1305- versus dofetilideinduced TdP were similar ( $311 \pm 18 \mathrm{~ms}$ versus $286 \pm 7 \mathrm{~ms}$, respectively, $\mathrm{P}=0.10$ ). 


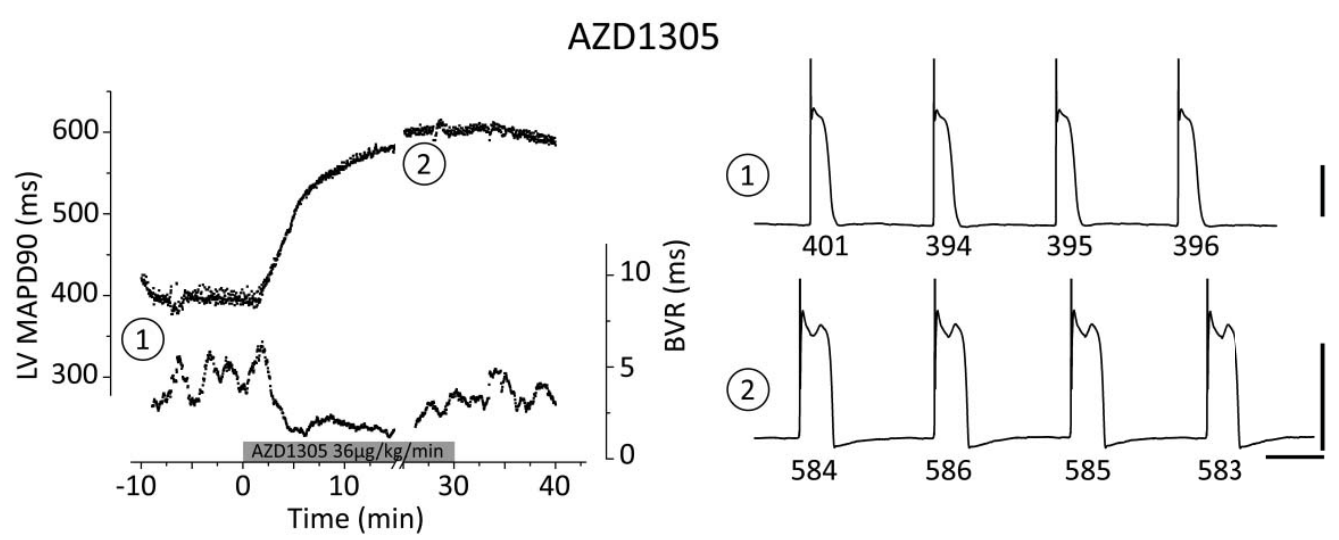

Dofetilide

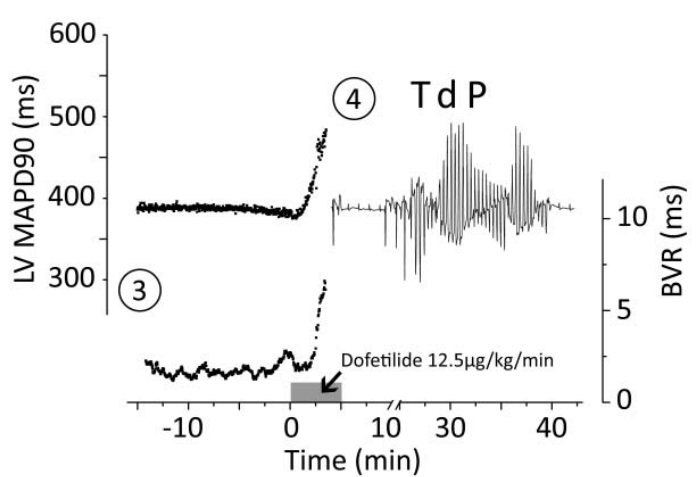

(3)

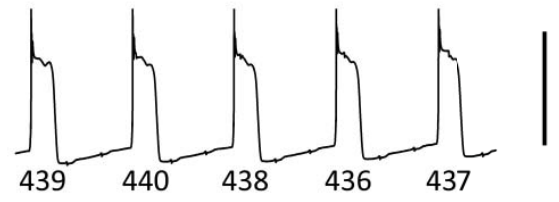

(4)

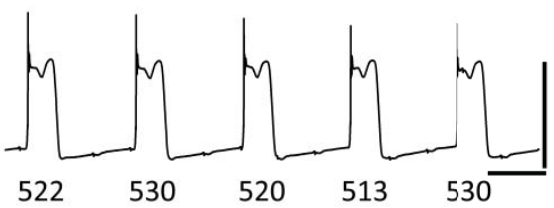

Figure 7: Panel (1), baseline. Panel (2), AZD1305. BVR is not increased in this animal after AZD1305. No arrhythmias were seen in this animal. Panel (3), baseline. Panel (4), dofetilide. BVR increased by dofetilide and TdP ensued after this. Right panels, LV MAPD90s (ms) are shown below signals. Vertical calibration bars, $10 \mathrm{mV}$. Horizontal calibration bars, $1 \mathrm{~s}$.

Figure 8 illustrates an example of TdP during infusion of AZD1305, occurring at 6.5 min. Spontaneous conversion from TdP to idioventricular rhythm occurred twice in this animal. 


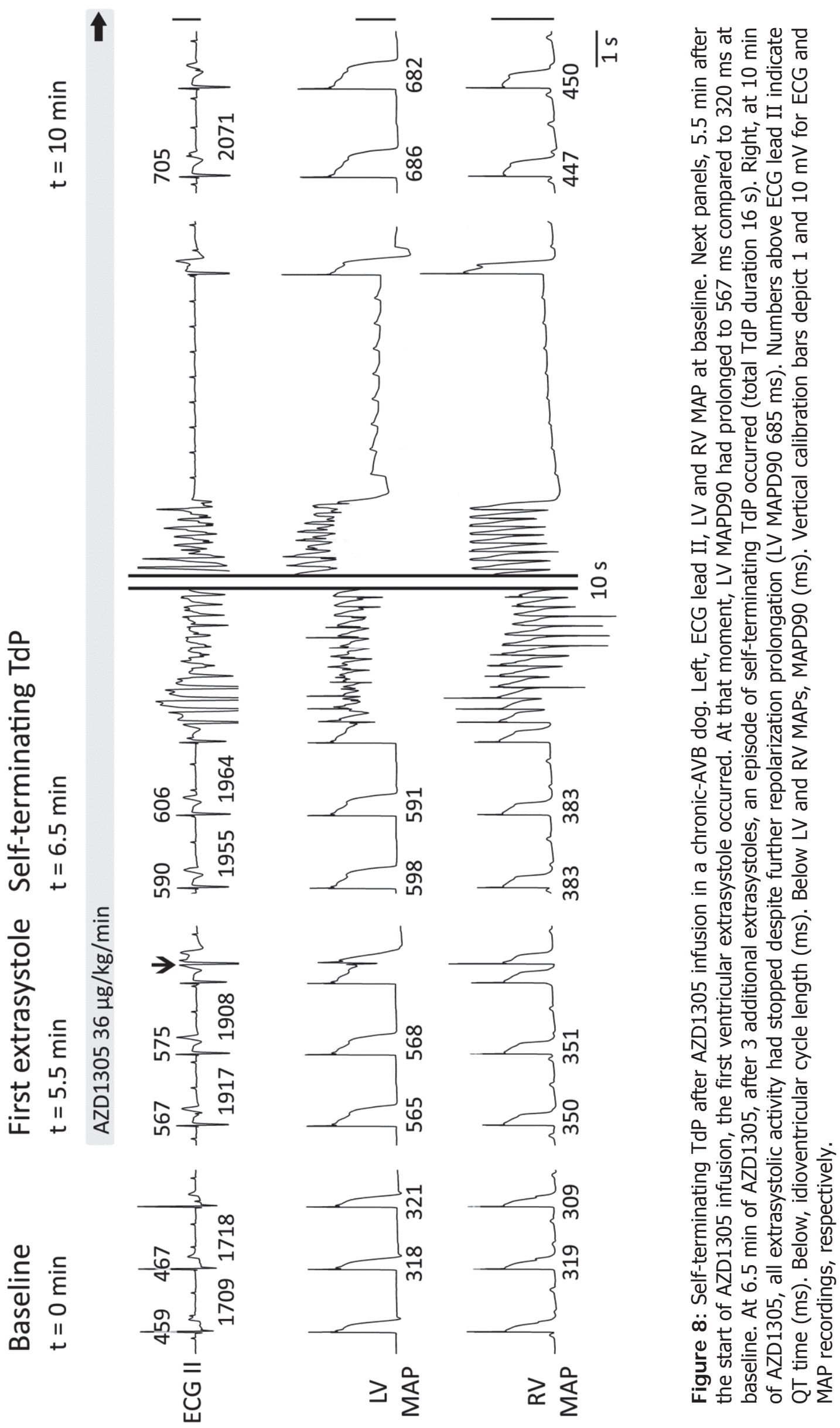




\subsubsection{Suppressive Effects of AZD1305 on Dofetilide-I nduced TdP}

In 4 chronic-AVB dogs with confirmed TdP induction by dofetilide in separate experiments, dofetilide was infused again after the administration of AZD1305 (Figure 1B). Under these conditions, first extrasystolic activity was postponed, occurring at $13 \pm 2.2 \mathrm{~min}$ after the start of dofetilide infusion (versus $3.8 \pm 0.2 \mathrm{~min}$ after dofetilide without AZD1305 pretreatment; $P<0.002)$. LV MAPD90 prolonged from $412 \pm 24 \mathrm{~ms}$ at baseline to $644 \pm 40 \mathrm{~ms}$ during AZD1305 ( $P=0.002$ versus baseline) to $684 \pm 49 \mathrm{~ms}$ after AZD1305 plus dofetilide ( $P=0.02$ versus AZD1305). In 2/4 animals, no TdP was seen at all, despite previous TdP susceptibility to dofetilide alone. In the other two animals, dofetilide after AZD1305 caused significantly fewer TdPs than during its solo administration: 1 (0-2) versus 7 (3-11) episodes, respectively $(P=0.02)$.

As an alternative regimen (Figure 1B), AZD1305 was administered as a fast bolus infusion $(870 \mu \mathrm{g} / \mathrm{kg})$ after the first dofetilide-induced $\operatorname{TdP}(n=4)$. Under these conditions, the number of TdP episodes was decreased (2 (1-3) versus 7 (3-11); $\mathrm{P}=0.05)$ compared to dofetilide alone.

\subsubsection{Atrial Effects of AZD1305 in the Normal and Remodeled Dog Heart}

The atrial effects of AZD1305 are illustrated in Figure 9. In the normal unremodeled heart, right-atrial MAPD50 ( $+31 \%$ from $137 \pm 6 \mathrm{~ms}$ at baseline, $\mathrm{P}=0.001$ ) and MAPD90 ( $+33 \%$ from $228 \pm 5$ ms, $P<0.001$ ) were significantly increased by AZD1305, as was the PP CL. These effects were very similar to those seen with dofetilide (Figure 10).

After chronic AVB, baseline right-atrial MAPD50, MAPD90 and PP intervals were similar as during sinus rhythm and atrial repolarization and PP $C L$ was still significantly prolonged by AZD1305 (Figure 9). For example, right-atrial MAPD90 was $223 \pm 9 \mathrm{~ms}$ at baseline versus $270 \pm 17 \mathrm{~ms}(P=0.03,+21 \%)$ at the end of AZD1305 infusion. Interestingly, even after remodeling dofetilide still led to significantly prolonged atrial repolarization (Figure 10). 
Sinus rhythm

Chronic AVB
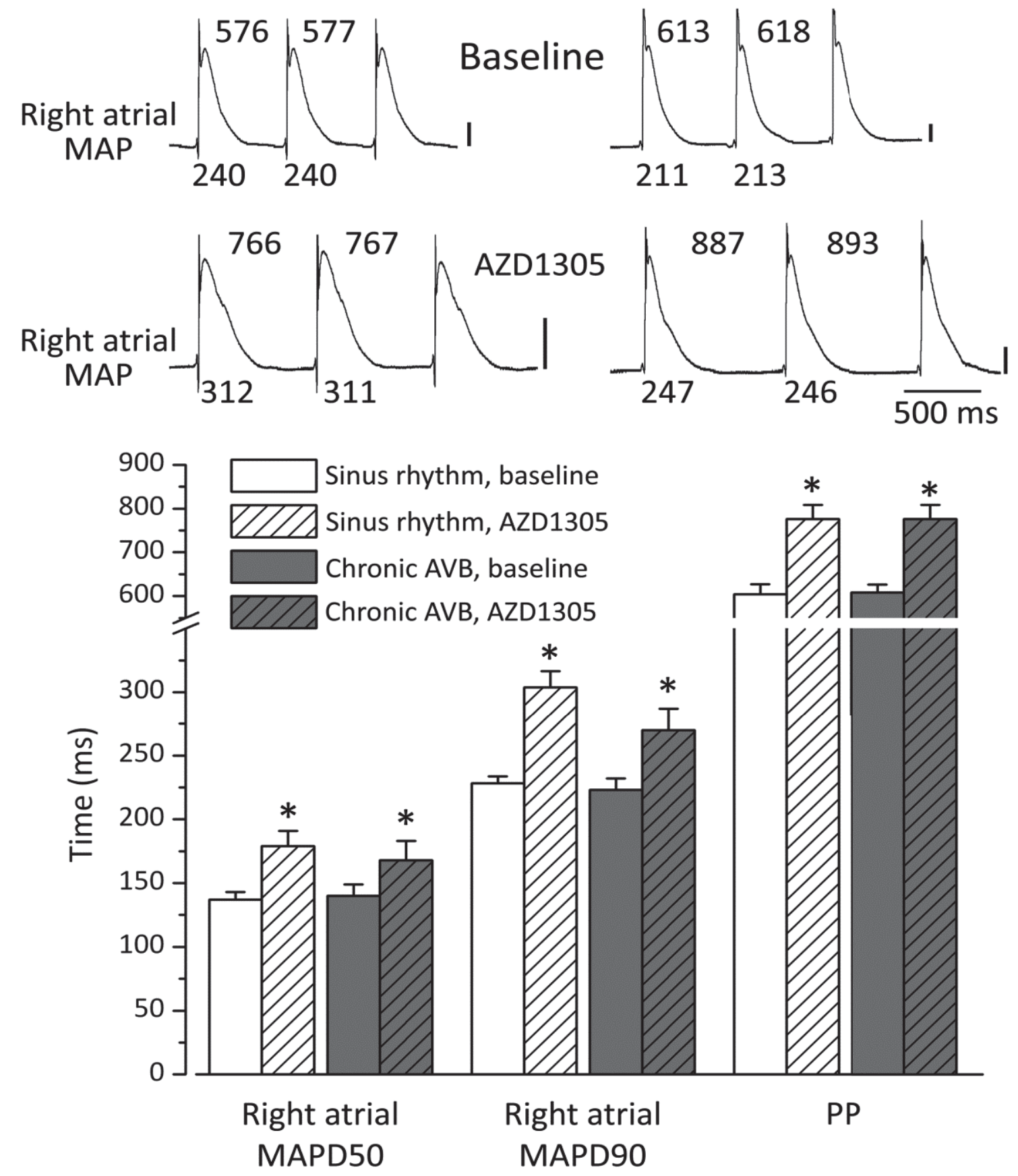

Figure 9: Atrial effects of AZD1305 in the normal and remodeled canine heart. Upper panels, Representative right-atrial MAPs at baseline and at the end of a 30-min infusion of AZD1305 in the same dog before (Sinus rhythm) and 3 weeks after AVB induction (Chronic AVB). Above the signals, cycle length (ms). Below, MAPD90s (ms). Lower panels, Average values for right-atrial MAPD50 and MAPD90, and PP intervals from 11 dogs. Vertical calibration bars, $10 \mathrm{mV} . *, \mathrm{P}<0.05$ versus baseline. 

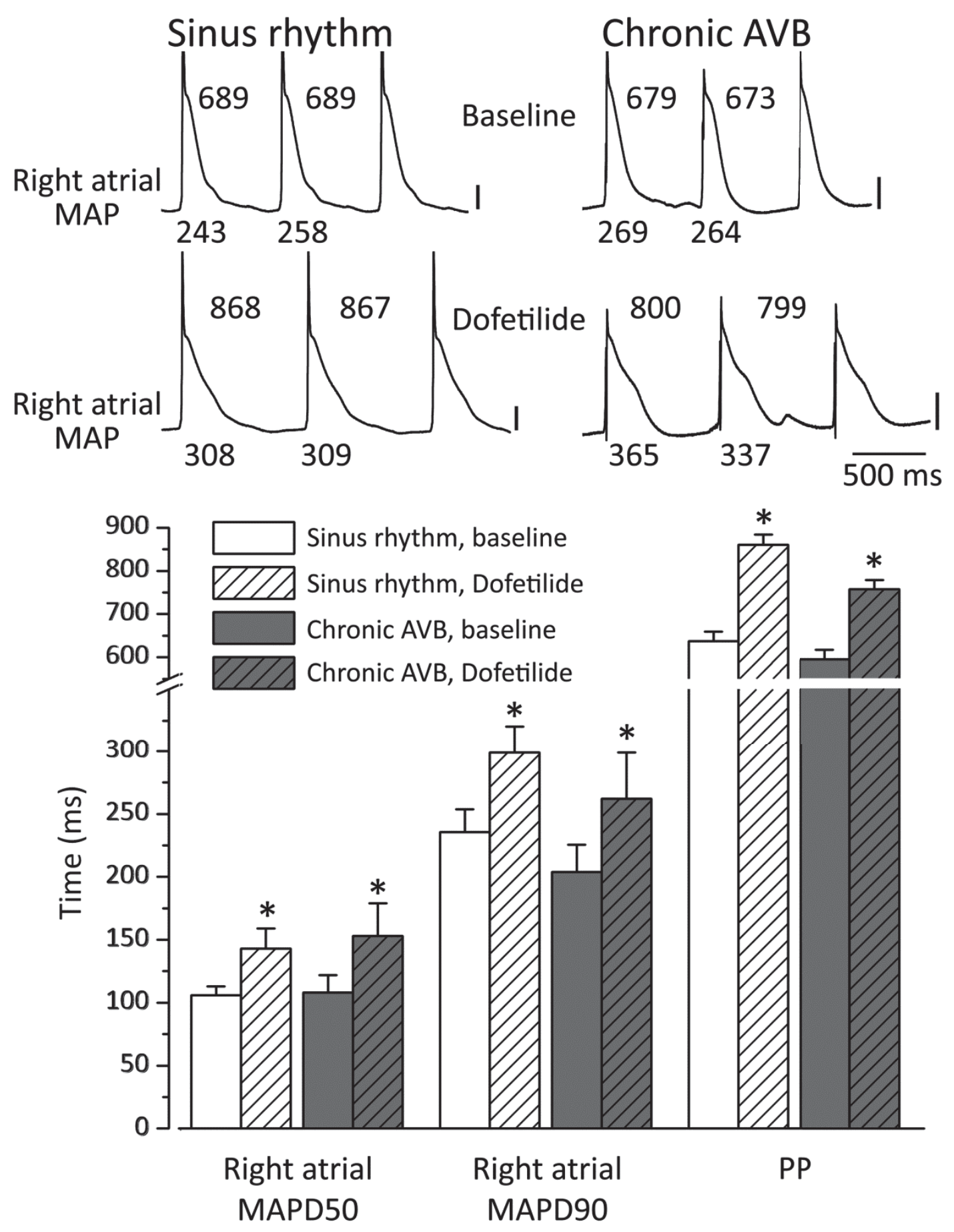

Figure 10: Atrial effects of dofetilide in the normal and remodeled canine heart. Upper panels, Representative right-atrial MAPs at baseline and at the end of a 5-min infusion of dofetilide in the same dog before (Sinus rhythm) and 3 weeks after AVB induction (Chronic AVB). Above the signals, cycle length (ms). Below, MAPD90s (ms). Lower panels, Average values for right-atrial MAPD50 and MAPD90, and PP intervals from 4 dogs. Vertical calibration bars, $10 \mathrm{mV}$. *, $\mathrm{P}<0.05$ versus baseline. 


\subsection{Discussion}

In this study we used the dog model with chronic AVB to compare in vivo cardiac electrophysiological effects, and any pro- and antiarrhythmic actions, of the novel investigational agent AZD1305 with dofetilide in the remodeled heart. Chemically, dofetilide is a methanesulphonamide derivative and thus a member of the same family of specific $\mathrm{I}_{\mathrm{Kr}}$-blocking antiarrhythmic agents such as ibutilide and d-sotalol (Figure 1A). AZD1305, on the other hand, is a disubstituted 9 oxabispidine compound structurally related to its congener AZD7009 (Figure 1A). Interestingly, the minor structural differences in AZD1305 vs AZD7009 introduce potent $\mathrm{I}_{\mathrm{CaL}}$-activity to the $I_{\mathrm{Kr}}$ and $I_{\mathrm{Na}}$-blocking characteristics described for both agents $7,8,17$. Hence, with its combined ion channel-blocking profile, AZD1305 has been proposed as an antiarrhythmic agent against AF with a lower proarrhythmic potential than other antiarrhythmic drugs. AZD1305 has been demonstrated to cause atrial-predominant effects in the healthy dog heart and attenuates ventricular repolarization-related instability caused by sudden rate changes during selective $\mathrm{I}_{\mathrm{Kr}}$ blockade ${ }^{8}$. In fact, AZD1305 was devoid of proarrhythmia in anesthetized methoxamine-sensitized rabbits, whereas other selective $I_{\mathrm{Kr}}$ blockers proved very torsadogenic in this animal model ${ }^{7,18}$.

In our experiments on anesthetized dogs with normal hearts, AZD1305 caused ventricular and atrial repolarization prolongation, but no repolarization instability or proarrhythmias. In chronic-AVB dogs with remodeled hearts and a high susceptibility to acquired TdP, AZD1305 was much less proarrhythmic than dofetilide, despite causing similar ventricular repolarization prolongation. If TdP occurred during AZD1305, the arrhythmia(s) arose early during the 30-min infusion phase, when the plasma concentrations were still rising. At $10 \mathrm{~min}$, the AZD1305 plasma concentration averaged $1.3 \pm 0.3 \mu \mathrm{mol} / \mathrm{L}$ ( $\mathrm{n}=6$ dogs with chronic AVB; Figure 4). In vitro findings indicate that AZD1305 has a higher potency against hERG (IC50 0.4 $\mu \mathrm{mol} / \mathrm{L}$ ) in this concentration range than against $\mathrm{I}_{\mathrm{CaL}}$ and hNav1.5 (IC50s of 1.2 and $1.5 \mu \mathrm{mol} / \mathrm{L}$, respectively) ${ }^{7}$. Although these in vitro findings can only be used with caution to predict potential effects in vivo, we speculate that the surge of ventricular extrasystoles and TdP during early AZD1305 infusion was due to initial proarrhythmic $\mathrm{I}_{\mathrm{Kr}}$ inhibition, followed by antiarrhythmic $\mathrm{I}_{\mathrm{CaL}}$ and $\mathrm{I}_{\mathrm{Na}}$ suppression upon mounting levels of the compound. Inhibition of either $I_{\mathrm{Na}}$ or $\mathrm{I}_{\mathrm{CaL}}$ has previously been shown to be effective in suppressing $\mathrm{I}_{\mathrm{Kr}}$-block-induced $\mathrm{TdP}$ in various experimental models. For example, $I_{\mathrm{Na}}$ block by lidocaine attenuated the incidence of TdP by almokalant in rabbits without influencing the almokalant-induced QT prolongation ${ }^{19}$. Similar effects have been shown in the chronic-AVB dog after dofetilide-induced TdP, both with lidocaine and the novel $\mathrm{I}_{\text {Natate }}$ blocker ranolazine ${ }^{20}$, although in the latter study multiple ventricular ectopic beats were not completely abolished. In the same animal model, Oros et al. ${ }^{21}$ showed a robust antiarrhythmic effect of both flunarizine and verapamil against dofetilide-induced TdP. To our knowledge, AZD1305 is the first compound, with the exception of amiodarone and dronedarone, with effects on $\mathrm{I}_{\mathrm{Kr}}$ 
together with ancillary dual $\mathrm{I}_{\mathrm{Na}}$ and $\mathrm{I}_{\mathrm{CaL}}$-blocking effects that has been investigated in this proarrhythmic model, and our results suggest that compounds with this profile could be devoid of proarrhythmic side effects if the ion-channel-blocking potencies are balanced (more so than for AZD1305), leaving no $\mathrm{I}_{\mathrm{kr}}$ block unattended.

\subsubsection{Repolarization Lability and Proarrhythmia Liability}

Various research groups have investigated the value of BVR parameters to predict imminent arrhythmia under conditions of repolarization lability. In the chronic-AVB dog, increases of beat-to-beat variability of MAPD have been shown to predict druginduced TdP ${ }^{22,}{ }^{23}$. Compounds increasing MAPD per se, but not BVR, are generally not torsadogenic in this experimental model. Beat-to-beat variability of the QT interval has also proven useful in heralding arrhythmia in other TdP models ${ }^{7,24,25}$. In the clinical setting, increased BVR of the QT interval characterized patients with drug-induced proarrhythmia, even in the absence of QTC prolongation ${ }^{26}$. Likewise, this parameter identified latent repolarization disorders in patients with congenital long QT syndrome ${ }^{27}$. BVR of the QT interval was also found to be increased in patients with dilated cardiomyopathy and heart failure, probably reflecting acquired repolarization disturbances and an increased susceptibility to sudden arrhythmic death ${ }^{28}$. In the present study, we have demonstrated that the combined inhibition of $\mathrm{I}_{\mathrm{CaL}}$ and $\mathrm{I}_{\mathrm{Na}}$ together with $\mathrm{I}_{\mathrm{Kr}}$ blockade does not significantly increase BVR in dogs with chronic AVB despite causing marked repolarization prolongation. These data reinforce the notion that $B V R$ is a robust predictor of $T d P$, more so than repolarization duration per se, at least under these experimental conditions.

\subsubsection{Atrial Effects of AZD1305 in the Remodeled Heart}

AZD1305 has been shown to be an effective antiarrhythmic agent in various animal models of $\mathrm{AF}^{9,10}$. It causes an atrial-predominant blockade of $\mathrm{I}_{\mathrm{Na}}$ (particularly tonic inhibition) which is suggested to translate into high antiarrhythmic efficacy ${ }^{9}$. Previously, the predecessor to AZD1305, AZD7009, was shown to restore sinus rhythm in up to $82 \%$ of patients with AF episodes lasting up to 30 days and with minimal proarrhythmic side effects ${ }^{29,} 30$. AZD7009 was less effective in patients with AF durations exceeding 1 month. A recent clinical study showed that AZD1305 was effective in converting $\mathrm{AF}$ to $\mathrm{SR}$, with a conversion rate of $50 \%$ within 90 min in the highest dose group, despite AF episode durations of up to 3 months ${ }^{12}$.

In the present study, AZD1305 led to significant increases in right-atrial MAPD50 and MAPD90 in both the normal and the AVB-remodeled dog heart (Figure 9). The mechanisms and consequences of atrial remodeling during chronic AVB are still incompletely understood. In an early study in dogs, right-atrial pressures were increased during acute and chronic overload ${ }^{31}$, thus promoting dilatation and hypertrophy. In goats, progressive atrial dilatation has been demonstrated in chronic 
$\mathrm{AVB}$, along with atrial hypertrophy, prolongation of induced AF paroxysms and local conduction delays ${ }^{32}$. Importantly, atria undergoing remodeling due to electricallyinduced AF were less responsive to $I_{k r}$ blockers given as solo administration ${ }^{33}$. The additional blockade of $\mathrm{I}_{\mathrm{Kur}}, \mathrm{I}_{\mathrm{TO}}$ and $\mathrm{I}_{\mathrm{KAch}}$, which prolonged the early atrial repolarization, led to restoration of the antiarrhythmic class-III action of the $\mathrm{I}_{\mathrm{Kr}}$ blocker in this goat model ${ }^{33}$. In other dog models, the myocardial substrate of AF in congestive heart failure appeared very different from that of rapid-atrial-pacing related $A F,{ }^{34}$ with contrasting efficacy of dofetilide ${ }^{35}$. Collectively, these data illustrate the impact of the type of remodeling on atrial drug responsiveness.

\subsubsection{Conclusions}

Despite causing a similar degree of repolarization delay, the combined ion-channel blocker AZD1305 induces less ventricular repolarization instability, reflected in LV $B V R$, and has a lower proarrhythmic potential than the selective $\mathrm{I}_{\mathrm{Kr}}$ blocker dofetilide in the chronic AVB-remodeled dog heart. AZD1305 can still lead to TdP in the remodeled heart, although in a minority of animals, during an infusion phase when plasma levels of the compound are still increasing. This is likely due to differential affinities of the drug for various ion channels, resulting in a window of preferential proarrhythmic $\mathrm{I}_{\mathrm{kr}}$ block. Further development of safer combined ion-channel blockers as antiarrhythmics could take this information into account.

\subsection{Acknowledgements}

The authors wish to Roel L.H.M.G. Spätjens, BSc, Cardiovascular Research Institute Maastricht, The Netherlands for invaluable help in figure preparation and technical assistance. D.M.J. was financially supported by AstraZeneca Ltd., United Kingdom. P.G.A.V. is supported by a Vidi grant from the Netherlands Organization for Scientific Research (ZonMw 91710365). 


\section{References}

(1) Benjamin EJ, Wolf PA, D'Agostino RB, Silbershatz H, Kannel WB, Levy D. Impact of atrial fibrillation on the risk of death: the Framingham Heart Study. Circulation. 1998; 98:946-5.

(2) Guo AS, Hylek EM, Phillips KA, Chang Y, Henault LE, Selby JV, Singer DE. Prevalence of diagnosed atrial fibrillation in adults: national implications for rhythm management and stroke prevention: the AnTicoagulation and Risk Factors in Atrial Fibrillation (ATRIA) Study. JAMA. 2001; 285:2370-5.

(3) Fedida D, Orth PM, Chen JY, Lin S, Plouvier B, Jung G, Ezrin AM, Beatch GN. The mechanism of atrial antiarrhythmic action of RSD1235. J CardiovasC Electrophysiol. 2005; 16:1227-38.

(4) Roy D, Pratt CM, Torp-Pedersen C, Wyse DG, Toft E, Juul-Moller S, Nielsen T, Rasmussen SL, Stiell IG, Coutu B, Ip JH, Pritchett EL, Camm AJ; Atrial Arrhythmia Conversion Trial Investigators. Vernakalant hydrochloride for rapid conversion of atrial fibrillation: a phase 3, randomized, placebo-controlled trial. Circulation. 2008; 117:1518-25.

(5) Dobrev D, Hamad B, Kirkpatrick P. Vernakalant. Nat Rev Drug Discov. 2010;9:915-6.

(6) Le Heuzey JY, De Ferrari GM, Radzik D, Santini M, Zhu J, Davy JM. A shortterm, randomized, double-blind, parallel-group study to evaluate the efficacy and safety of dronedarone versus amiodarone in patients with persistent atrial fibrillation: the DIONYSOS study. J Cardiovasc Electrophysiol. 2010; 21:597-605.

(7) Carlsson L, Andersson B, Linhardt G, Löfberg L. Assessment of the ion channel-blocking profile of the novel combined ion channel blocker AZD1305 and its proarrhythmic potential versus dofetilide in the methoxaminesensitized rabbit in vivo. J Cardiovasc Pharmacol. 2009; 54:82-9.

(8) Andersson B, Abi-Gerges N, Carlsson L. The combined ion channel blocker AZD1305 attenuates late $\mathrm{Na}$ current and $\mathrm{I}_{\mathrm{kr}}$-induced action potential prolongation and repolarization instability. Europace. 2010; 12:1003-10.

(9) Burashnikov A, Zygmunt AC, Di Diego JM, Linhardt G, Carlsson L, Antzelevitch C. AZD1305 exerts atrial predominant electrophysiological actions and is effective in suppressing atrial fibrillation and preventing its reinduction in the dog. J Cardiovasc Pharmacol. 2010; 56:80-90. 
(10) Sicouri S, Carlsson L, Antzelevitch C. Electrophysiologic and antiarrhythmic effects of AZD1305 in canine pulmonary vein sleeves. J Pharmacol Exp Ther. 2010; 334:255-9.

(11) Toivonen L, Raatikainen P, Walfridsson H, Englund A, Hegbom F, Anfinsen OG, Gjesdal K, Pehrson S, Johansson S, Frison L, Berggren AR, Edvardsson N. A Randomized, Invasive Cardiac Electrophysiology Study of the Combined ion Channel Blocker AZD1305 in Patients After Catheter Ablation of Atrial Flutter. J Cardiovasc Pharmacol. 2010; 56:300-8.

(12) Rónaszéki A, Alings M, Egstrup K, Gaciong Z, Hranai M, Király C, Sereg M, Figatowski W, Bondarov $\mathrm{P}$, Johansson S, Frison L, Edvardsson N, Berggren A. Pharmacological cardioversion of atrial fibrillation--a double-blind, randomized, placebo-controlled, multicentre, dose-escalation study of AZD1305 given intravenously. Europace. 2011; 13:1148-56.

(13) Volders PG, Sipido KR, Vos MA, Kulcsár A, Verduyn SC, Wellens HJ. Cellular basis of biventricular hypertrophy and arrhythmogenesis in dogs with chronic complete atrioventricular block and acquired torsade de pointes. Circulation. 1998; 98:1136-47.

(14) Volders PG, Sipido KR, Vos MA, Spätjens RL, Leunissen JD, Carmeliet E, Wellens $\mathrm{HJ}$. Downregulation of delayed rectifier $\mathrm{K}^{+}$currents in dogs with chronic complete atrioventricular block and acquired torsades de pointes. Circulation. 1999; 100:2455-61.

(15) Stengl M, Ramakers C, Donker DW, Nabar A, Rybin AV, Spätjens RL, van der Nagel T, Wodzig WK, Sipido KR, Antoons G, Moorman AF, Vos MA, Volders PG. Temporal patterns of electrical remodeling in canine ventricular hypertrophy: focus on $\mathrm{I}_{\mathrm{Ks}}$ downregulation and blunted beta-adrenergic activation. Cardiovasc Res. 2006; 72:90-100.

(16) Van de Water A, Verheyen J, Xhonneux R, Reneman RS. An improved method to correct the QT interval of the electrocardiogram for changes in heart rate. J Pharmacol Methods. 1989; 22:207-17.

(17) Persson F, Carlsson L, Duker G, Jacobson I. Blocking characteristics of hERG, hNav1.5, and hKvLQT1/hminK after administration of the novel anti-arrhythmic compound AZD7009. J Cardiovasc Electrophysiol. 2005;15:1444-1450. 
(18) Carlsson L, Abrahamsson C, Andersson B, Duker G, Schiller-Linhardt G. Proarrhythmic effects of the class III agent almokalant: importance of infusion rate, QT dispersion, and early afterdepolarisations. Cardiovasc Res. 1993; 27:2186-93.

(19) Carlsson L, Drews L, Duker G, Schiller-Linhardt G. Attenuation of proarrhythmias related to delayed repolarization by low-dose lidocaine in the anesthetized rabbit. J Pharmacol Exp Ther. 1993; 267:1076-80.

(20) Antoons G, Oros A, Beekman JD, Engelen MA, Houtman MJ, Belardinelli L, Stengl M, Vos MA. Late $\mathrm{Na}^{+}$current inhibition by ranolazine reduces torsades de pointes in the chronic atrioventricular block dog model. J Am Coll Cardiol. 2010; 55:801-9.

(21) Oros A, Houtman MJ, Neco P, Gomez AM, Rajamani S, Oosterhoff P, Attevelt NJ, Beekman JD, van der Heyden MA, Ver Donck L, Belardinelli L, Richard S, Antoons G, Vos MA; CONTICA investigators. Robust anti-arrhythmic efficacy of verapamil and flunarizine against dofetilide-induced TdP arrhythmias is based upon a shared and a different mode of action. Br J Pharmacol. 2010; 161:162-75.

(22) Thomsen MB, Verduyn SC, Stengl M, Beekman JD, de Pater G, van Opstal J, Volders PG, Vos MA. Increased short-term variability of repolarization predicts d-sotalol-induced torsades de pointes in dogs. Circulation. 2004; 110:2453-9.

(23) Thomsen MB, Beekman JD, Attevelt NJ, Takahara A, Sugiyama A, Chiba K, Vos MA. No proarrhythmic properties of the antibiotics Moxifloxacin or Azithromycin in anaesthetized dogs with chronic-AV block. $\mathrm{Br} \mathrm{J}$ Pharmacol. 2006; 149:1039-48.

(24) Lengyel C, Varró A, Tábori K, Papp JG, Baczkó I. Combined pharmacological block of $\mathrm{I}_{\mathrm{Kr}}$ and $\mathrm{I}_{\mathrm{Ks}}$ increases short-term QT interval variability and provokes torsades de pointes. Br J Pharmacol. 2007; 151:941-51.

(25) Jacobson I, Carlsson L, Duker G. Beat-by-beat QT interval variability, but not QT prolongation per se, predicts drug-induced torsades de pointes in the anaesthetised methoxamine-sensitized rabbit. J Pharmacol Toxicol Methods. $2011 ; 63: 40-6$.

(26) Hinterseer M, Thomsen MB, Beckmann BM, Pfeufer A, Schimpf R, Wichmann $\mathrm{HE}$, Steinbeck G, Vos MA, Kääb S. Beat-to-beat variability of QT intervals is increased in patients with drug-induced long-QT syndrome: a case control pilot study. Eur Heart J. 2008; 29:185-90. 
(27) Hinterseer M, Beckmann BM, Thomsen MB, Pfeufer A, Dalla Pozza R, Loeff M, Netz H, Steinbeck G, Vos MA, Kääb S. Relation of increased short-term variability of QT interval to congenital long-QT syndrome. Am J Cardiol. 2009; 103:1244-8.

(28) Hinterseer M, Beckmann BM, Thomsen MB, Pfeufer A, Ulbrich M, Sinner MF, Perz S, Wichmann HE, Lengyel C, Schimpf R, Maier SK, Varró A, Vos MA, Steinbeck G, Kääb S. Usefulness of short-term variability of QT intervals as a predictor for electrical remodeling and proarrhythmia in patients with nonischemic heart failure. Am J Cardiol. 2010; 106:216-20.

(29) Crijns HJ, Van Gelder IC, Walfridsson H, Kulakowski P, Rónaszéki A, Dedek V, Malm A, Almgren $O$. Safe and effective conversion of persistent atrial fibrillation to sinus rhythm by intravenous AZD7009. Heart Rhythm. 2006; 3:1321-31.

(30) Geller JC, Egstrup K, Kulakowski P, Rosenqvist M, Jansson MA, Berggren A, Edvardsson N, Sager $P$, Crijns $H J$. Rapid conversion of persistent atrial fibrillation to sinus rhythm by intravenous AZD7009. J Clin Pharmacol. 2009; 49:312-22.

(31) Turina M, Baboti I, Bussmann WD, Krayenbühl HP. Haemodynamics of acute and chronic atrioventricular block in dogs. Cardiovasc Res. 1969; 3:209-17.

(32) Neuberger HR, Schotten U, Verheule S, Eijsbouts S, Blaauw Y, van Hunnik A, Allessie $M$. Development of a substrate of atrial fibrillation during chronic atrioventricular block in the goat. Circulation. 2005; 111:30-7.

(33) Blaauw Y, Schotten U, van HA, Neuberger HR, Allessie MA. Cardioversion of persistent atrial fibrillation by a combination of atrial specific and non-specific class III drugs in the goat. Cardiovasc Res. 2007; 75: 89-98.

(34) Li D, Fareh S, Leung TK, Nattel S. Promotion of atrial fibrillation by heart failure in dogs: atrial remodeling of a different sort. Circulation. 1999; 100: 87-95.

(35) Li D, Bénardeau A, Nattel S. Contrasting efficacy of dofetilide in differing experimental models of atrial fibrillation. Circulation. 2000; 102: 104-12. 


\section{Chapter 7}

The Electro-Mechanical Window in Anaesthetized

Guinea-Pigs: A New Marker for Torsades de Pointes

Risk Screening

British Journal of Pharmacology; 2012; 166(2):689-70 


\begin{abstract}
QT prolongation is commonly used as a surrogate marker for Torsades de Pointes $(\mathrm{TdP})$ risk of non-cardiovascular drugs. However, the use of this indirect marker often leads to misinterpretation of the realistic TdP risk, as tested compounds may cause QT prolongation without effectively evoking TdP in man. A negative electromechanical (E-M) window has recently been proposed as an alternative risk marker for TdP in a canine LQT1 model. The current work studied the E-M window in anaesthetized guinea-pigs as a screening marker for TdP in man. The effects of various reference drugs and changes in body temperature on the E-M window were assessed in instrumented guinea-pigs. The E-M window was defined as the delay between the duration of the electrical (QT interval) and mechanical (QLVPend) systole. Drugs with documented TdP liability (quinidine, haloperidol, domperidone, terfenadine, thioridazine and dofetilide) consistently decreased the E-M window, whereas compounds with no TdP risk in man (salbutamol and diltiazem) failed to affect the E-M window. Interestingly, drugs with documented clinical QT prolongation, but with low risk for TdP (amiodarone, moxifloxacin and ciprofloxacin) did not decrease the E-M window. Furthermore, the E-M window was minimally affected by changes in heart rate or body temperature. In conclusion a decreased E-M window was consistently observed with drugs documented to have high TdP risk, but not with drugs with low or no TdP risk. These results suggest that the E-M window in anaesthetized guinea-pigs is a risk marker for TdP in man.
\end{abstract}




\subsection{I ntroduction}

Delayed ventricular repolarization - whether by congenital defects such as the long QT syndrome (LQTS) ${ }^{1}$ or by the effects of potassium channels blocking drugs such as terfenadine or dofetilide - is associated with a high incidence of Torsades de Pointes (TdP) and sudden cardiac death. The incidence of TdP with cardiac proarrhythmic drugs is $1-8 \%$ while the incidence with non-cardiac drugs is considerably lower (approximately 1 in 100000$)^{2}$. Because of its infrequent occurrence TdP is typically not seen in clinical trials. As such, QT interval prolongation has become a surrogate marker of TdP risk and is commonly used as a preclinical and clinical biomarker for pro-arrhythmic activity of non-cardiovascular drugs ${ }^{3}$.

Currently QT prolongation is a major safety issue in drug development and a dedicated regulatory guideline (ICH S7B) describes strategies for identification of drug candidates affecting cardiac repolarization. Since no single assay is sufficient to predict the risk of TdP in humans, the ICH S7B document recommends an integrated approach including at least an in vitro assessment of inhibition of the rapidly activating delayed rectifier potassium current $\left(\mathrm{I}_{\mathrm{Kr}_{\mathrm{r}}}\right)$ and an in vivo QT-assay. In line with the ICH S7B guideline, frontloading of cardiovascular safety data, i.e. screening for TdP liability early on in drug development has become common practice. In this view, guinea-pigs have received a great deal of attention with regards to evaluation of effects of newly discovered drugs on the QT interval at the early stage. Indeed, the anesthetized guinea-pig model is a useful tool for "first in line" in vivo cardiovascular safety assessment due to the limited amounts of test compound required; the similarity of cardiac ionic currents between guineapigs and man ${ }^{4}$, and the ease of manipulation of guinea-pigs. Over the last years many reference drugs have been evaluated in the anaesthetized guinea-pig model and the predictive value of this model for QT prolongation in humans is well documented ${ }^{5,6,7}$.

Although the current strategies based on identification of drug-induced QT prolongation in animal models (ICH S7B) and its clinical counterpart - the thorough QT study (ICH E14) - have been relatively successful in the attrition of unsafe drug candidates, this approach inherently generates a number of false positive results due to the lack of direct association between the surrogate marker (QT interval) and the final endpoint (TdP risk). Indeed, the magnitude of QTC prolongation does not correlate with the TdP risk in man; and several drugs prolong the QT interval without causing TdP in man ${ }^{2,8,9}$. 
Over the last years a number of new risk markers and models for TdP risk evaluation have been proposed, illustrating the absolute need of the pharmaceutical industry to develop more accurate markers for TdP risk in man. The current state-of-the-art of risk markers and pro-arrhythmia models are comprehensively described elsewhere ${ }^{8,10,11}$. The electro-mechanical (E-M) window has recently been proposed as one of these alternative risk markers for TdP in man.

The current work aimed to evaluate the E-M window in anaesthetized guinea-pigs as a new risk marker for TdP. The E-M window is defined as the delay between the electrical (QT-interval) and mechanical $\left(\mathrm{QLVP}_{\text {end }}\right)$ systole $\left(\mathrm{E}-\mathrm{M}\right.$ window $=\mathrm{QLVP}_{\text {end }}$ interval - QT interval, Figure 1). Despite intensive studies on E-M coupling in man since the 1980's, the concept of disturbed E-M coupling as a risk marker for TdP was only recently introduced in safety pharmacology ${ }^{12}$. In healthy individuals, the duration of the electrical systole (QT interval) is shorter than, but closely parallels the duration of mechanical systole (measured indirectly by the time between the $\mathrm{Q}$ wave and the second heart sound; QS2) throughout the normal range of resting heart rates ${ }^{13}$. Interestingly, changes in autonomic tone ${ }^{14}$ or high circulating catecholamine levels ${ }^{15}$ were reported to invert the normal QT/QS2 ratio. Furthermore, the QT/QS2 ratio was found to be a useful indicator for several cardiovascular diseases ${ }^{16,17,18}$. Moreover, patients with the Romano-Ward inherited long QT syndrome were found to have an altered QT/QS2 ratio ${ }^{19}$. Recently, van der Linde et al. (2010) ${ }^{12}$ showed that in a canine LQT1 dog model an inverted (negative) E-M window is a prerequisite for the initiation of TdP. A negative E-M window is a highly pro-arrhythmic condition, due to the fact that under these conditions there is a mismatch of electrical and mechanical activity. This provides a situation where arrhythmias can ensue, most likely because $\mathrm{Ca}^{2+}$ can continue to enter the cardiac myocytes favoring the occurrence of $\mathrm{Ca}^{2+}$ overload, leading to both early and delayed afterdepolarizations as well as aftercontractions which may eventually lead to TdP. Restoration of the E-M window by atenolol, a $\beta$-adrenergic receptor ( $\beta A R$ ) antagonist, or verapamil, an $\mathrm{L}$-Type $\mathrm{Ca}^{2+}$ channel blocker, prevented the induction of TdP by $\mathrm{I}_{\mathrm{Ks}}$ blockade in combination with $\beta A R$ stimulation ${ }^{12}$.

Based on these observations we hypothesized that a negative E-M window in the anaesthetized guinea-pig model might be a (LQT1) risk marker of TdP in man. Implementation of such a new risk marker for TdP in an early screening platform, like the anaesthetized guinea-pig model, would mean a significant breakthrough in cardiovascular safety pharmacology. In an attempt to document the predictive value of the E-M window in anaesthetized guinea-pigs the effects of various pharmacological agents with different torsadogenic profiles (high, low and no TdP risk) were assessed. 


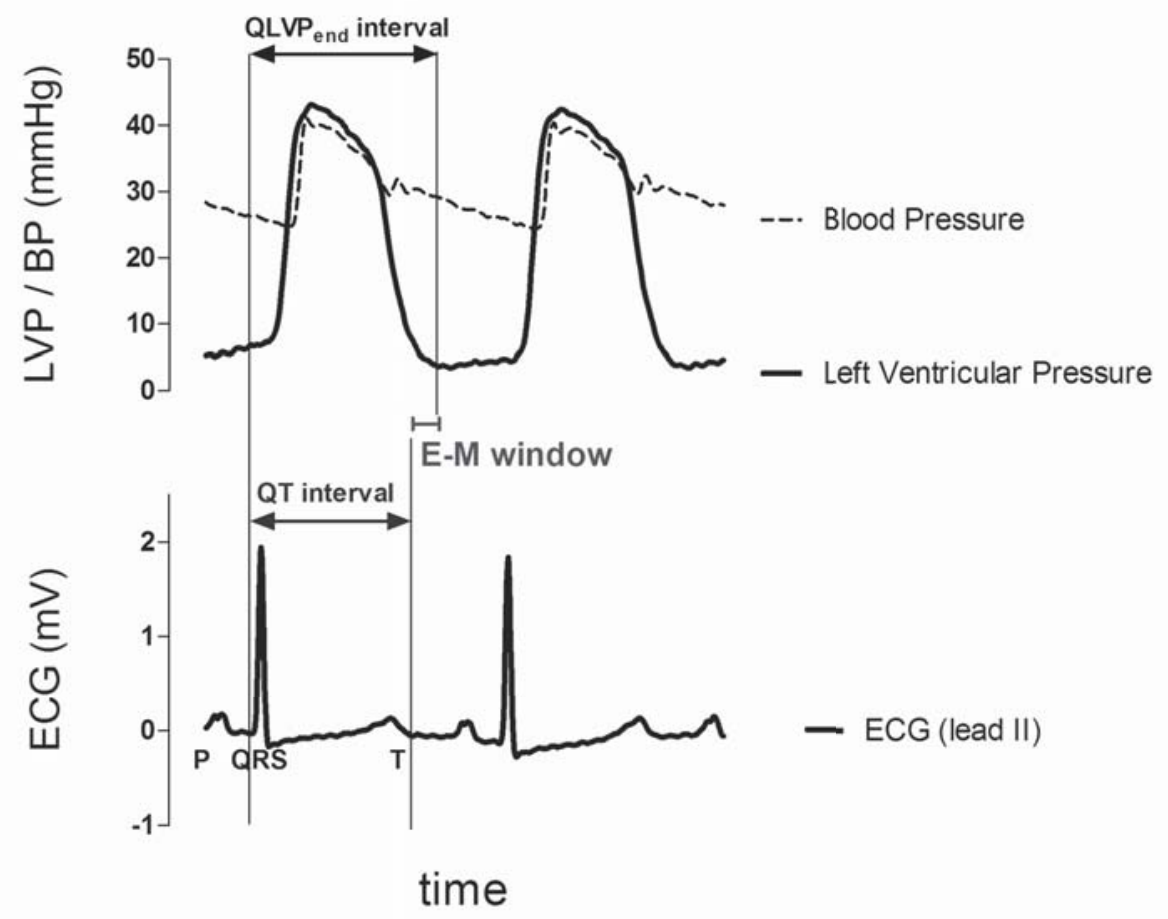

Figure 1: The Wiggers diagram illustrates the synchronization of the electrical (electrocardiogram, ECG) and mechanical (left ventricular pressure, LVP) activity of the cardiac muscle in anaesthetized guinea-pigs. The electro-mechanical window (E-M window) is defined as the delay between the duration of the electrical (QT interval) and mechanical $\left(\mathrm{QLVP}_{\text {end }}\right.$ interval) systole. E-M window $=\mathrm{QLVP}_{\text {end }}$ interval $-\mathrm{QT}$ interval.

\subsection{Methods}

\subsubsection{Animal Use and Care}

The studies were approved by the Ethical Committee of Vito (Mol, Belgium) and conformed to the Guide for the Care and Use of Laboratory Animals published by the US National Institute of Health (NIH Publication No. 85-23, revised 1996).

Female Dunkin Hartley guinea-pigs (weight 380-540 g) were purchased from Charles Rivers (L'Arbresle, France). Animals arrived at the animal facilities at least 1 week before the study and were housed in groups (up to 5 animals per cage; $>3500 \mathrm{~cm}^{2}$ per cage) with access to food and water ad libitum.

\subsubsection{Surgical Procedure}

Animals were anaesthetized by intraperitoneal administration of sodium pentobarbital (60 mg/kg). A tracheotomy was performed and the guinea-pigs were mechanically ventilated with room air (model '683' respirator, Harvard Apparatus, Les Ulis, France) using a stroke volume of $10 \mathrm{ml} / \mathrm{kg}$ at a rate of 60 strokes/min. The tracheal tube was connected to a pressure transducer to monitor the pulmonary inflation pressure throughout the experiment. Animals were placed on an electrical heat pad at $37^{\circ} \mathrm{C}$ 
(small operating table, Harvard Apparatus). Both jugular veins were cannulated for respectively intravenous drug administration (right jugular vein) and continuous infusion of pentobarbital ( $6 \mathrm{mg} / \mathrm{h}$ ) to maintain anaesthesia (left jugular vein). An open-lumen catheter was inserted in the left carotid artery for blood pressure measurement and collection of arterial blood samples. A tip catheter (SPR-249, Millar Instruments, Houston, Texas, USA) was positioned in the left ventricle for measurement of left ventricular pressure (accessed via the right carotid artery). Four needle electrodes were put subcutaneously to record the electrocardiogram (ECG). Pressure and ECG signals were relayed to a bio-amplifier (EMKA Technologies, Paris, France) with output to a data acquisition system (IOX, EMKA Technologies). Raw data were captured at sampling rates of $1000 \mathrm{~Hz}$ (ECG signals) or $500 \mathrm{~Hz}$ (blood pressure and left ventricular pressure). After being instrumented animals were allowed to stabilize for a period of at least $20 \mathrm{~min}$.

\subsubsection{Experimental Protocol}

The effect of various reference drugs on cardio-electrophysiological and cardiohemodynamic parameters was assessed in anaesthetized guinea-pigs. Guinea-pigs received consecutively five increasing doses of the test compounds. All compounds were infused intravenously at a rate of $1 \mathrm{ml} / \mathrm{kg}$ over a 10-min infusion period with 5min intervals between consecutive doses. Animals in the separate vehicle group were infused equal volumes of saline. At the end of the vehicle experiments dofetilide was infused over a 1-min period as positive control.

In addition, a separate set of experiments $(n=3)$ were performed to assess the effect of temperature (range $38^{\circ} \mathrm{C}-34^{\circ} \mathrm{C}$ ) on the cardiovascular parameters. After stabilization the heating pad was switched-off and temperature (measured via a rectal probe) and associated cardiovascular parameters were monitored during the cooling phase.

\subsubsection{Data Analysis}

Analysis of raw data was performed using ECG-Auto software (version 2.5.1, EMKA Technologies). ECG signals (lead II) were automatically analyzed based on shape-recognition linked to a user-defined library of ECG waveforms. The QTCB interval was calculated for every single beat by using the previous RR interval. The end of the ventricular relaxation (LVPend point) was defined by applying a threshold $(-100 \mathrm{mmHg} / \mathrm{s})$ on the first derivative of the relaxation part of the LVP signal. The RR interval, PQ interval, QRS interval, QT interval, QTcB interval, QLVP end $_{\text {interval and }}$ the E-M window were calculated beat by beat. The accuracy of the analysis was reviewed by manual over-reading. For each minute-interval, median values were calculated and used for reporting. 
The QT interval, the QLVP ${ }_{\text {end }}$ interval and the E-M window were reported in actual units (ms), whereas for QTcB the changes from baseline in percentage were calculated. The parameters 60 seconds prior to the first drug administration were defined as baseline values. $(t=0 \mathrm{~min}$ ). The results from individual animals were pooled by treatment group and visualized in graphs as mean \pm .

\subsubsection{Chemicals}

Clinical reference substances were purchase as dry powder. Quinidine, haloperidol, domperidone, terfenadine and diltiazem were purchased from Tocris Bioscience (Bristol, United Kingdom); moxifloxacin, ciprofloxacin, thioridazine, isoprenaline and salbutamol were purchased from Sigma (Bornem, Belgium). The dry powder (taking into account a correction factor for the different molecular weights of various salt forms) was dissolved in a vehicle consisting of physiological saline solution. Vehicles of terfenadine and domperidone contained $10 \%$ hydroxypropyl- $\beta$ cyclodextrin to increase solubility. Amiodarone was purchased in a clinically available formulation (Cordarone $\mathbb{R} 150 \mathrm{mg}$ per $3 \mathrm{ml}$, Sanofi Aventis) and was diluted with saline.

\subsubsection{Statistics}

Results were expressed as mean \pm SEM. Changes from baseline (in actual units) were analyzed by a one-way ANOVA test with Bonferroni post-hoc test.

\subsection{Results}

Various pharmacological agents were administered intravenously to instrumented and anaesthetized guinea-pigs. Following drug administration the duration of the QT interval and the QLVP $_{\text {end }}$ interval was continuously monitored. In addition, the E-M window, defined as the delay between the electrical and mechanical systole (E-M window $=\mathrm{QLVP}_{\text {end }}$ interval $-\mathrm{QT}$ interval) was calculated beat by beat (Figure 1).

\subsubsection{Drugs with Well-Documented TdP Risk}

Drugs associated with prolongation of the QT interval and with well-documented TdP risk in man (quinidine $(n=4)$, haloperidol $(n=3)$, domperidone $(n=4)$, terfenadine $(n=4)$, thioridazine $(n=5)$ and dofetilide (positive control, $n=4)$ all showed dosedependent prolongation of the QT and QTCB interval (Figure 2 and Figure 3). The QLVP $_{\text {end }}$ interval was not prolonged to the same extent as the QT interval resulting in a dose-dependent decrease of the E-M window. Administration of quinidine, haloperidol and domperidone eventually lead to a negative E-M window (Figure 3), whereas terfenadine and thioridazine decreased the E-M window, but failed to make 
this parameter negative (Table 1). Interestingly, quinidine decreased the E-M window, but a slight restoration of the E-M window between consecutive doses was observed, which was not observed for the QTCB interval. Terfenadine caused marked bradycardia and hypotension at the highest dose $(10 \mathrm{mg} / \mathrm{kg})$ thereby generating less reliable results at this dose.

\subsubsection{Negative Controls (No TdP Risk)}

Intravenous administration of vehicle (saline, $n=4$ ) did not affect the $E-M$ window or the QTCB interval (Figure 2 and Figure 4). Administration of the positive control (dofetilide) caused a negative E-M window and prolongation of the QTcB interval, illustrating the validity of the assay. In addition, two clinical drugs with no TdP risk were tested: salbutamol $(n=5)$ and diltiazem $(n=4)$. Both drugs failed to affect the E-M window. Salbutamol caused a drastic increase in heart rate (data not shown) thereby significantly decreasing the QT and QLVP end interval. The QTCB interval was shortened by salbutamol illustrating the limitations of Bazett's formula for heart rate correction, whereas the E-M window was hardly influenced by the strong changes in heart rate. Diltiazem caused a decrease of heart rate (data not shown) and eventually cardiovascular collapse at $3 \mathrm{mg} / \mathrm{kg}$ and animals died the highest dose $10 \mathrm{mg} \mathrm{kg}^{-1}$. However, the E-M window was not altered by diltiazem administration.

\subsubsection{Amiodarone, Moxifloxacin and Ciprofloxacin}

Amiodarone $(\mathrm{n}=4)$ and moxifloxacin $(\mathrm{n}=4)$ showed remarkable results (Figure 2 and Figure 5). Both drugs caused a significant prolongation of the QTCB interval of respectively $12 \%$ and $14 \%$. However, since the QLVP end interval prolonged to the same (moxifloxacin) or higher (amiodarone) extent no negative E-M window was observed after administration of these compounds. Furthermore, dofetilide failed to evoke a negative E-M window following amiodarone administration. Ciprofloxacin $(n=4)$ showed a tendency for QT prolongation (5\% increase) at the highest dose, but the E-M window was not affected by ciprofloxacin administration.

\subsubsection{I soprenaline}

Isoprenaline $(n=4)$ showed a shortening in the QT interval, as would be expected due to the chronotopic effects of this compound. At the same time a decrease of the QLVP $_{\text {end }}$ interval was observed, which was not completely synchronized with the effects on QT. This led to a transient decrease in the E-M window at the highest concentrations (Figure 2 and Figure 4). 

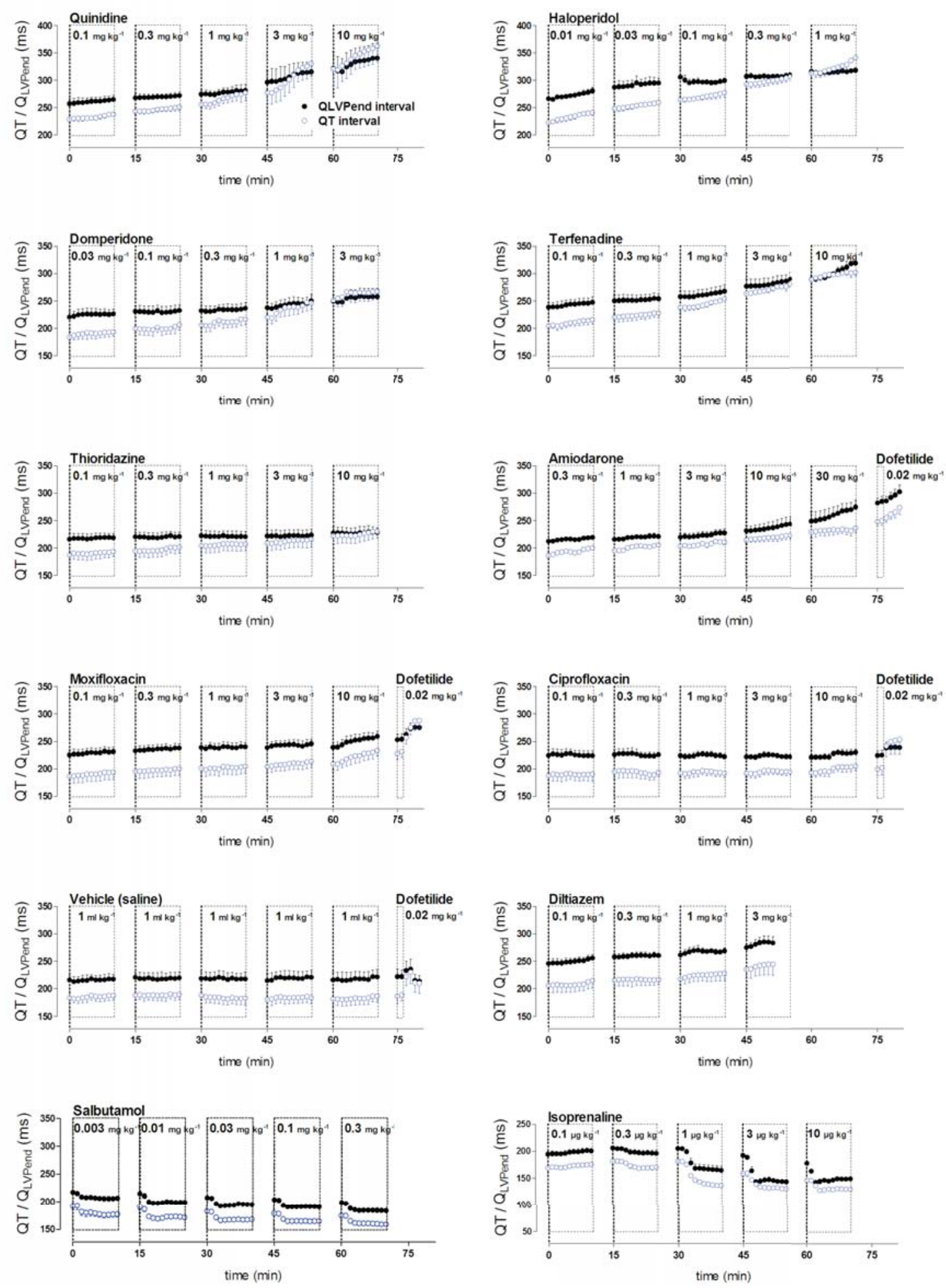

Figure 2: Graphs show the effect of various pharmacological agents on the electromechanical coupling in anaesthetized guinea-pigs. Drugs with high TdP risk (quinidine, haloperidol, domperidone, terfenadine, dofetilide and thioridazine) induced unequal changes

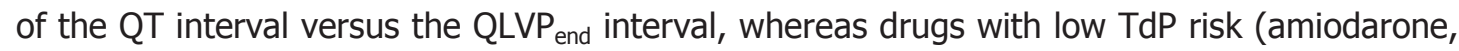
moxifloxacin and ciprofloxacin), with no TdP risk (vehicle, salbutamol and diltiazem) induced parallel changes of the QT interval and LVP end interval. Finally, isoprenaline showed different

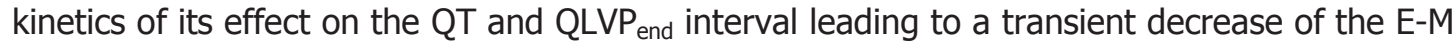
window. Plots show mean \pm SEM. 

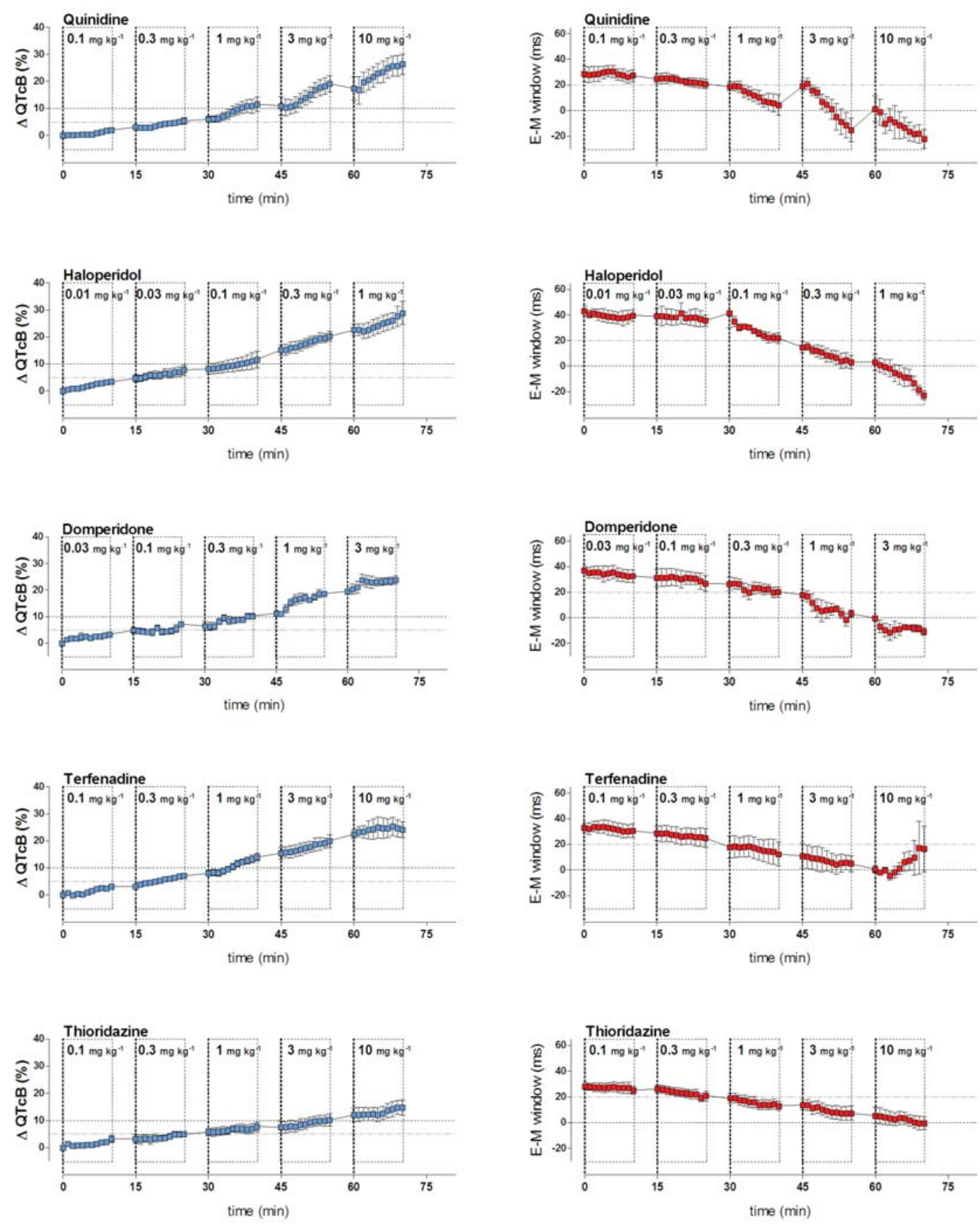

Figure 3: Graphs show the effects of drugs with documented TdP risk on the commonly used surrogate marker for TdP risk (QTCB, \% change from baseline; left panels) and on the $\mathrm{E}-\mathrm{M}$ window (ms; right panels). Quinidine, haloperidol, domperidone, terfenadine and ioridazine prolonged the QTCB interval and consistently decreased the E-M window. Graphs show mean \pm SEM. 

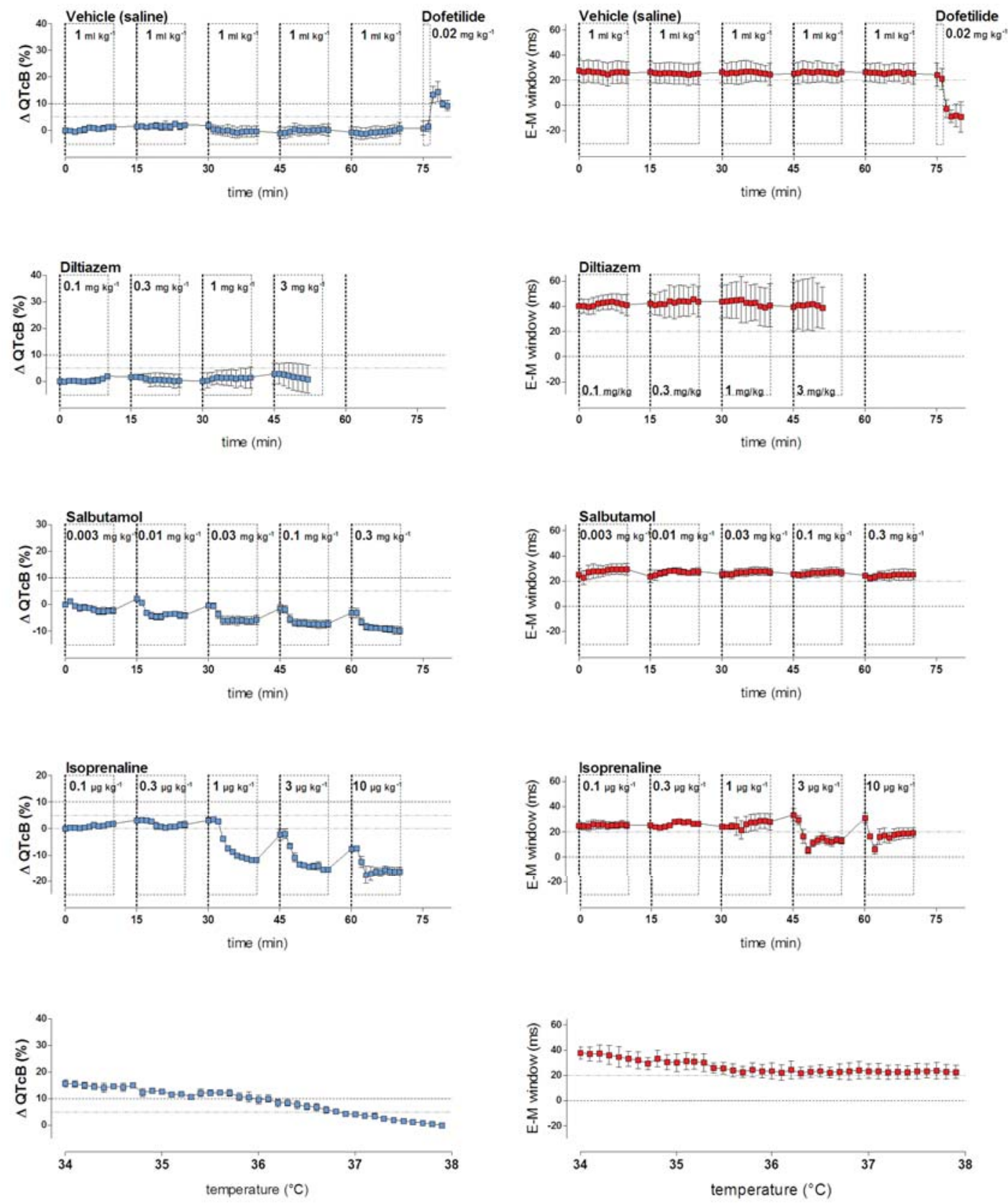

Figure 4: Plots show the effects of vehicle, of drugs with no TdP risk (diltiazem, salbutamol), of isoprenaline and of changes in body temperature (lowest graphs) on the commonly used surrogate marker for TdP risk (QTcB, \% change from baseline; left panels) and on the E-M window (ms; right panels). The vehicle did not cause QTCB prolongation or shortening of the E-M window. Salbutamol and diltiazem, respectively, increased and decreased heart rate (data not shown), but did not affect the E-M window. Isoprenaline transiently reduced the E-M window due to the different kinetics of its effect on the QT and QLVP end interval. Finally, cooling of anaesthetized animals from $38^{\circ} \mathrm{C}$ to $34^{\circ} \mathrm{C}$ prolonged the QTcB interval $\left(-14.6 \mathrm{~ms}^{\circ} \mathrm{C}^{-1}\right)$, but had no major effect on the duration of the E-M window. Graphs show mean \pm SEM. 

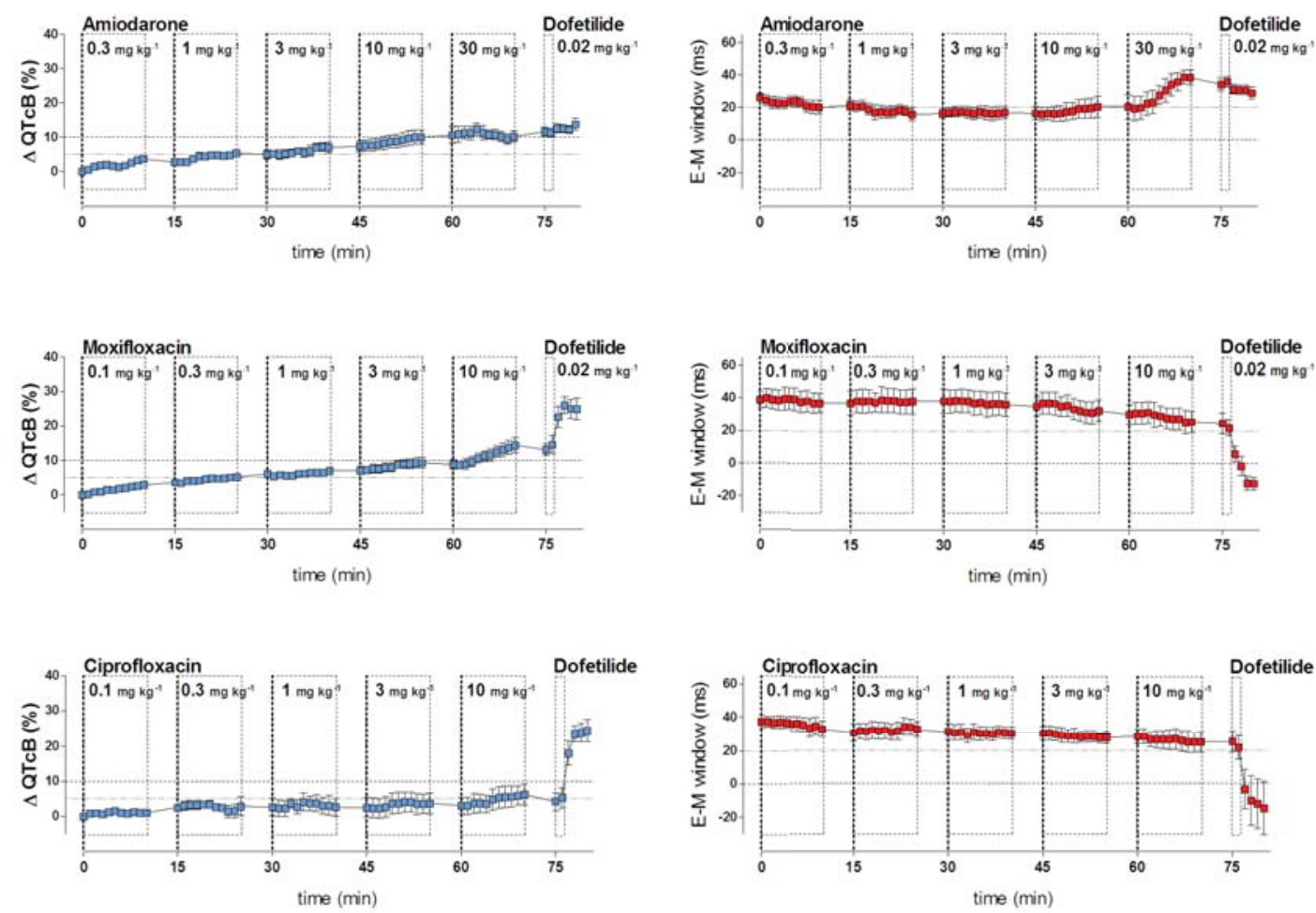

Figure 5: Graphs show the effects of amiodarone and two antibiotics (moxifloxacin and ciprofloxacin) on the commonly used surrogate marker for TdP risk (QTCB, \% change from baseline; left panels) and on the E-M window (ms; right panels). Amiodarone increased the QTCB interval, but did not decrease the E-M window; in fact, it significantly increased the E-M window and prevented induction of a negative $\mathrm{E}-\mathrm{M}$ window by dofetilide. Moxifloxacin and ciprofloxacin hardly affected the E-M window despite prolongation of the QTCB interval. Graphs show mean \pm SEM.

\subsubsection{Effect of Temperature}

The effect of temperature on the electro-mechanical coupling was investigated in a different set of experiments ( $n=3$, Figure 4). Cooling of the animals from $38^{\circ} \mathrm{C}$ to $34^{\circ} \mathrm{C}$ caused increases of the QT interval, the QTcB interval (slope: $-14.6 \mathrm{~ms}^{\circ}{ }^{\circ} \mathrm{C}^{-1}$ ) and the QLVP end interval. The E-M window remained stable during cooling; and even slightly increased when cooling below $35.5^{\circ} \mathrm{C}$. 
Table 1

\begin{tabular}{|c|c|c|c|c|c|c|c|}
\hline \multicolumn{2}{|c|}{ Change from baseline (ms) } & \multirow{2}{*}{$\begin{array}{c}\text { baseline } \\
\text { (actual } \\
\text { units) }\end{array}$} & \multirow{2}{*}{$\begin{array}{c}\text { dose } 1 \\
10 \mathrm{~min}\end{array}$} & $\begin{array}{l}\text { dose } 2 \\
25 \mathrm{~min}\end{array}$ & \multirow{2}{*}{$\begin{array}{l}\text { dose } 3 \\
40 \mathrm{~min}\end{array}$} & \multirow{2}{*}{$\begin{array}{l}\text { dose } 4 \\
55 \mathrm{~min}\end{array}$} & \multirow{2}{*}{$\begin{array}{l}\text { dose } 5 \\
70 \mathrm{~min}\end{array}$} \\
\hline & & & & & & & \\
\hline quinidine & $\begin{array}{l}\text { E-M } \\
\text { window }\end{array}$ & $28.4 \pm 5.9$ & $-1.2 \pm 1.7$ & $-7.7 \pm 5.7$ & $-24.2 \pm 8.8$ & $-43.6 \pm 9.9 * *$ & $-50.4 \pm 8.9 * *$ \\
\hline $\mathrm{n}=4$ & QTcB & $403.0 \pm 6.2$ & $7.9 \pm 0.6$ & $21.6 \pm 4.3$ & $46.5 \pm 10.1 *$ & $76.5 \pm 11.4 * * *$ & $105.9 \pm 14.2 * * *$ \\
\hline haloperidol & $\begin{array}{l}\text { E-M } \\
\text { window }\end{array}$ & $43.2 \pm 4.0$ & $-3.6 \pm 0.7$ & $-7.4 \pm 2.2$ & $-21.3 \pm 7.8$ & $-39.8 \pm 7.7 * *$ & $-66.0 \pm 7.5^{* * *}$ \\
\hline $\mathrm{n}=3$ & QTcB & $407.4 \pm 6.8$ & $13.7 \pm 2.7$ & $31.4 \pm 7.2$ & $47.2 \pm 11.8 *$ & $82.5 \pm 6.6 * * *$ & $117.2 \pm 15.8 * * *$ \\
\hline domperidone & $\begin{array}{l}\text { E-M } \\
\text { window }\end{array}$ & $37.3 \pm 3.4$ & $-4.5 \pm 1.7$ & $-10.5 \pm 2.9$ & $-17.0 \pm 3.8$ & $-34.0 \pm 4.6^{* * * *}$ & $-48.0 \pm 5.6^{* * *}$ \\
\hline $\mathrm{n}=4$ & QTcB & $369.3 \pm 10.0$ & $11.8 \pm 2.8$ & $26.2 \pm 3.9 *$ & $37.5 \pm 4.3^{* * * *}$ & $68.9 \pm 4.8 * * *$ & $88.3 \pm 7.1 * * *$ \\
\hline terfenadine & $\begin{array}{l}\text { E-M } \\
\text { window }\end{array}$ & $32.8 \pm 4.0$ & $-2.3 \pm 2.1$ & $-7.9 \pm 3.8$ & $-20.4 \pm 5.3$ & $-28.0 \pm 2.2$ & $-12.6 \pm 17.9$ \\
\hline$n=4$ & QTcB & $385.9 \pm 5.2$ & $10.6 \pm 2.4$ & $25.5 \pm 2.1^{*}$ & $54.5 \pm 3.7 * * *$ & $79.8 \pm 6.1 * * *$ & $92.5 \pm 9.7 * * *$ \\
\hline thioridazine & $\begin{array}{l}\text { E-M } \\
\text { window }\end{array}$ & $28.2 \pm 3.0$ & $-3.0 \pm 1.3$ & $-7.4 \pm 2.0$ & $-15.2 \pm 3.9$ & $-20.9 \pm 6.8^{*}$ & $-28.6 \pm 6.8^{* *}$ \\
\hline $\mathrm{n}=5$ & QTcB & $364.9 \pm 11.6$ & $11.5 \pm 4.2$ & $18.0 \pm 2.9$ & $27.6 \pm 6.0^{*}$ & $36.9 \pm 7.8 * *$ & $53.1 \pm 9.3 * * *$ \\
\hline vehicle & $\begin{array}{l}\text { E-M } \\
\text { window }\end{array}$ & $27.7 \pm 9.2$ & $-1.7 \pm 0.7$ & $-2.3 \pm 0.9$ & $-3.2 \pm 0.4$ & $-1.0 \pm 2.6$ & $-2.3 \pm 1.8$ \\
\hline $\mathrm{n}=4$ & QTcB & $365.3 \pm 9.1$ & $4.6 \pm 2.2$ & $7.5 \pm 3.5$ & $-0.9 \pm 7.2$ & $0.7 \pm 7.9$ & $3.0 \pm 7.9$ \\
\hline & $\begin{array}{l}\text { E-M } \\
\text { window }\end{array}$ & $25.0 \pm 5.3$ & $4.4 \pm 3.2$ & $2.6 \pm 2.7$ & $2.2 \pm 2.9$ & $1.6 \pm 3.4$ & $0.1 \pm 2.6$ \\
\hline $\mathrm{n}=5$ & QTcB & $372.1 \pm 6.2$ & $-8.6 \pm 4.4$ & $-15.7 \pm 3.9$ & $-21.6 \pm 6.9$ & $-27.3 \pm 5.5^{* *}$ & $-36.5 \pm 5.8 * * *$ \\
\hline & $\begin{array}{l}\text { E-M } \\
\text { window }\end{array}$ & $40.2 \pm 6.0$ & $0.8 \pm 4.9$ & $3.4 \pm 11.0$ & $0.5 \pm 16.4$ & $-28.0 \pm 2.2$ & $-12.6 \pm 17.9$ \\
\hline $\mathrm{n}=4$ & QTcB & $382.9 \pm 17.2$ & $7.1 \pm 2.9$ & $0.7 \pm 8.9$ & $5.6 \pm 14.4^{* * * *}$ & $79.8 \pm 6.1 * * *$ & $92.5 \pm 9.7$ \\
\hline & $\begin{array}{l}\text { E-M } \\
\text { window }\end{array}$ & $26.3 \pm 2.3$ & $-6.3 \pm 1.9$ & $-10.5 \pm 1.8$ & $-9.5 \pm 2.2$ & $-6.0 \pm 4.4$ & $12.0 \pm 2.4$ \\
\hline $\mathrm{n}=4$ & QTcB & $373.1 \pm 2.3$ & $13.6 \pm 3.6$ & $19.9 \pm 3.2$ & $26.1 \pm 5.9 *$ & $37.5 \pm 7.7 * *$ & $38.2 \pm 6.0^{* *}$ \\
\hline moxifloxacin & $\begin{array}{l}\text { E-M } \\
\text { window }\end{array}$ & $39.0 \pm 5.0$ & $-2.0 \pm 0.9$ & $-1.0 \pm 2.8$ & $-3.0 \pm 2.4$ & $-6.8 \pm 2.8$ & $-13.5 \pm 3.3 *$ \\
\hline $\mathrm{n}=4$ & $\mathrm{QTcB}$ & $364.6 \pm 12.9$ & $10.6 \pm 1.1$ & $18.8 \pm 0.6$ & $25.4 \pm 1.7 *$ & $33.6 \pm 4.9 * *$ & $52.0 \pm 6.4^{* * *}$ \\
\hline ciprofloxacin & $\begin{array}{l}\text { E-M } \\
\text { window }\end{array}$ & $37.5 \pm 3.8$ & $-4.3 \pm 1.3$ & $-4.5 \pm 1.9$ & $-7.0 \pm 2.0$ & $-9.8 \pm 1.3$ & $-12.4 \pm 4.0$ \\
\hline$n=4$ & QTcB & $367.5 \pm 13.7$ & $4.0 \pm 1.0$ & $9.7 \pm 9.6$ & $8.5 \pm 9.3$ & $12.5 \pm 10.5$ & $22.0 \pm 10.5$ \\
\hline
\end{tabular}

Table 1: Baseline values (actual units) and change from baseline for the E-M window and the QTCB interval after administration of various clinical reference drugs. Data show mean \pm SEM. A one-way ANOVA with Bonferoni post-hoc test was performed on the 'change from baseline' values, $* \mathrm{P}<0.05, * * \mathrm{P}<0.01, * * * \mathrm{P}<0.001$.

\subsubsection{Occurrence of Arrhythmias}

None of the reference drugs evoked episodes of TdP. Isolated ventricular premature contractions (VPCs) and an atrial premature contraction (APC) were observed in 8 and 1 out of 45 animals respectively. Most of these VPC's were observed during replacement of the catheters and were not observed in later experiments where the catheters were appropriately fixated. Right bundle branch block ( $r$-BBB) was reported after administration of diltiazem ( 1 of 4 ), terfenadine (4 of 4 ), haloperidol (1 of 3), thioridazine (2 of 5) and amiodarone (1 of 4). Furthermore atrio-ventricular block (AVB) was observed after treatment with diltiazem (3 of 4 animals), terfenadine ( 1 of 4 ), haloperidol ( 1 of 3 ) and dofetilide (2 of 4 ). Finally, diltiazem caused complete cardiovascular collapse in all animals (starting from $3 \mathrm{mg} / \mathrm{kg}$ ) eventually leading to death. 


\subsection{Discussion}

\subsubsection{The Anaesthetized Guinea-Pig Model and the E-M Window}

Over the last years the anaesthetized guinea-pig model has received a great deal of attention as a useful tool for early in vivo cardiovascular safety screening. Guineapigs have a cardiac electrophysiology that largely resembles human and the small size of the animals favors the use of this model for cardiovascular safety screening. Several anaesthesia regimens have been described for assessing QT prolongation in guinea-pigs 20, 21, 22 with the pentobarbital anaesthetized model being the most common and best documented $5,6,7,23$. In addition to the ECG-derived information (QT interval, QRS interval and PQ interval) the anaesthetized guinea-pig model provides information on hemodynamics (heart rate, blood pressure, QA interval), cardiac performance (left ventricular pressure) and on the induction of spontaneous arrhythmias making it a versatile cardiovascular screening model (Figure 6). Recently, the E-M window has been proposed as a novel risk marker for TdP in a LQT1 dog model ${ }^{12}$. The present work provides the first documented report of the E-M window as a general risk marker for TdP within an early screening model.

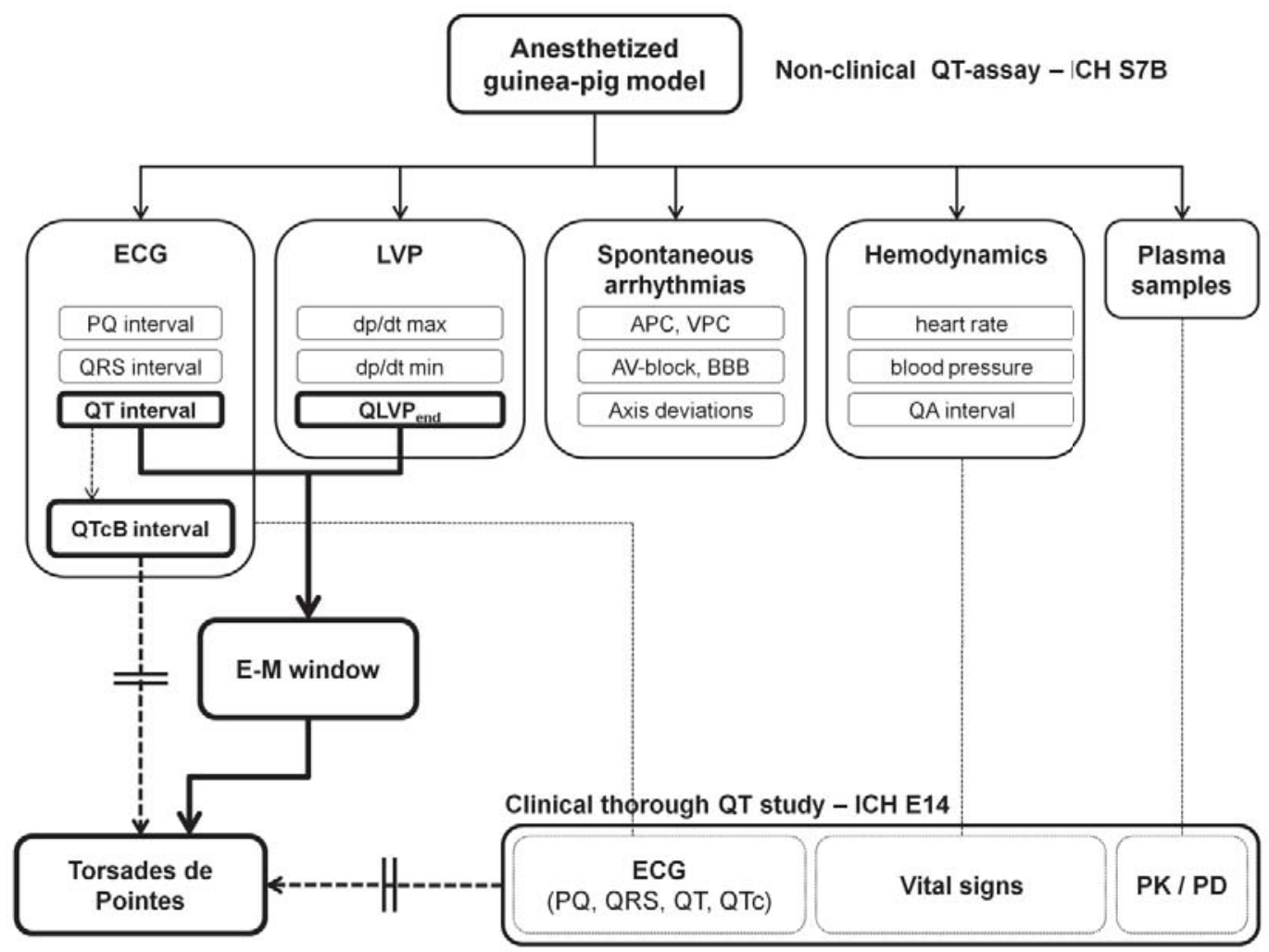

Figure 6: Figure shows a schematic overview of the anaesthetized guinea-pig model as a versatile early cardiovascular safety screening tool. At present, both pre-clinical (ICH S7B) and clinical (ICH E14) studies use the QTc interval as a surrogate marker for TdP risk in man. The E-M window provides a new risk marker for TdP risk in man that might eventually be implemented in clinical research. APC, atrial premature contraction; VPC, ventricular premature contraction; BBB, bundle branch block (left or right). 


\subsubsection{Drugs with High TdP Risk are Selectively Flagged}

Drugs with documented high TdP risk ${ }^{24,25}$ consistently decreased the E-M window. Quinidine, domperidone, haloperidol and dofetilide caused a negative E-M window that might form a substrate for the development of TdP. Due to a large repolarization reserve in guinea-pigs ${ }^{26,}{ }^{27} \mathrm{I}_{\mathrm{Kr}}$ blockade by itself did not elicit TdP in the anaesthetized guinea-pig model.

The results of terfenadine and thioridazine are less clear. Despite a substantial decrease of the E-M window, terfenadine and thioridazine did not cause negative $\mathrm{E}-\mathrm{M}$ windows on their own. This might suggest that additional challenges on the repolarization reserve are required to trigger TdP with these agents. Terfenadine, for example, has been shown to be a multi-ion channel blocker, affecting both inward and outward currents, including the L-type calcium current $\left(\mathrm{I}_{\mathrm{CaL}}\right)$ and the slowly activating delayed rectifier potassium current $\left(\mathrm{I}_{\mathrm{Kr}}\right)$, which could be an explanation for the absence of a negative E-M window with this compound ${ }^{28}$. Interestingly, terfenadine did not trigger TdP in a phenylephrine-sensitized rabbit model, whereas halofantrine and clofilium did lead to $\mathrm{TdP}^{29}$.

In the anaesthetized guinea-pig model a $5 \%$ increase in the QTcB interval can be accurately detected ${ }^{30}$ and a $10 \%$ prolongation of the QTCB interval should be classified as important QT prolongation. Similarly, based on our current experience we suggest that a shortening of the E-M window below 20 ms warrants further evaluation, whereas a negative E-M window points directly to high TdP risk in man. Drugs with no TdP risk (nor the vehicle) had no effect on the E-M window demonstrating the selectivity of the new risk marker.

\subsubsection{Amiodarone}

The multi-target agent amiodarone has extremely complex electropharmacological actions, with effects on multiple ion channels as well as $\beta A R s^{31}$, and is an interesting agent in the context of QT prolongation and TdP, despite causing a modest prolongation of the QT interval, it is an effective antiarrhthymic drug with a relatively low potential for TdP. Amiodarone has been found safe in patients who had experienced TdP while taking other drugs ${ }^{32,33}$ and can be used to treat $\operatorname{TdP}^{34}$, although it has various off target side effects which can limit chronic administration in a large cohort of patients. Furthermore, amiodarone showed relatively low potential for TdP in the canine chronic AV-block model, after both chronic ${ }^{35}$ and acute dosing ${ }^{36}$ and also in the Screenit rabbit model ${ }^{37}$. In line with these reports, acute dosing of amiodarone did not elicit a negative E-M window in our experiments. Moreover, amiodarone increased the $\mathrm{E}-\mathrm{M}$ window in anaesthetized guinea-pigs and prevented inversion of the E-M window by dofetilide. A similar protective effect of amiodarone has been described in a setting of sotalol-induced arrhythmias in dogs ${ }^{38}$. 
The mechanism of this protective effect might be the conservation of a positive E-M window, most likely due to the alterations in $\mathrm{Ca}^{2+}$ handling, although a reduction in heterogeneity of cardiac repolarization has been attributed to amiodarone as well ${ }^{39}$.

\subsubsection{Moxifloxacin and Ciprofloxacin}

Fluoroquinolones antibiotics are all associated with some degree of QT prolongation ${ }^{40,41}$, but the different members of this therapeutic class have different torsadogenic profiles ${ }^{42}$. Grepafloxacin (withdrawn from the market in 1999) and sparfloxacin have a high risk for induction of TdP in humans ${ }^{43}$, whereas ciprofloxacin and moxifloxacin have a low risk for TdP in man. In agreement with the use of moxifloxacin as a positive control in thorough QT studies ${ }^{44}$; and consistent with published pre-clinical data moxifloxacin prolonged the QTCB interval ${ }^{6,45}$ the present study. Despite considerably prolonging the QTCB interval, moxifloxacin only caused a minor decrease in the E-M window in anaesthetized guinea-pig suggesting minimal risk for TdP at the maximal dose used in this study $(10 \mathrm{mg} / \mathrm{kg})$. In line with these results moxifloxacin $(8 \mathrm{mg} / \mathrm{kg}$ ) did not produce episodes of TdP in a canine chronic AV-block model ${ }^{46}$. Whether higher doses of moxifloxacin would further compromise the E-M window is not known, but pharmacokinetics of moxifloxacin (400 mg, oral administration, once daily) are well-controlled which could explain why only few cases of moxifloxacin-related TdP have been reported. For ciprofloxacin no effect on the E-M window was observed. This favorable safety outcome in the anaesthetized guinea-pig model is in line with the frequent use of ciprofloxacin in clinical practice as a safe antibiotic.

Taken together, the E-M window appears to classify drugs with high and low TdP risk correctly and might be a valuable addition to the classical QTc interval screening.

\subsubsection{I soprenaline}

Isoprenaline transiently reduced the E-M window due to the different kinetics of its effect on the QT and QLVP end interval. The decrease of the E-M window by isoprenaline might explain the torsadogenic activity of isoprenaline in the LQT1 dog model (with compromised $\mathrm{I}_{\mathrm{KS}}$ ) ${ }^{12}$. However, in anaesthetized guinea-pigs combined administration of $\mathrm{I}_{\mathrm{Kr}^{-}}$(quinidine $10 \mathrm{mg} / \mathrm{kg}$ ) and $\mathrm{I}_{\mathrm{ks}}$-blockers (JNJ303 $2 \mathrm{mg} / \mathrm{kg}$ ) - resulting in large negative E-M windows (less than -50 ms) - did not result in spontaneous or $\beta A R$ stimulation (isoprenaline $0.0125 \mathrm{mg} / \mathrm{kg}$ ) induced TdP (unpublished data). As such, no direct association between a negative E-M window and initiation of TdP could currently be demonstrated in guinea-pigs. However, in the canine LQT1 model the necessity of a negative E-M window for the onset of TdP has been demonstrated ${ }^{12}$. 


\subsubsection{Effect of Heart Rate and Body Temperature on the E-M Window}

The duration of the QT interval is dependent on several factors including heart rate, autonomic influences ${ }^{47}$ and body temperature ${ }^{48}$. Different correction formulas have been applied in an attempt to discriminate between heart rate dependent and heart rate independent changes of the QT interval. In anaesthetized guinea-pigs the Bazett's formula is frequently applied to correct for heart rate ${ }^{6,20}$, and this correction has been shown to be the most effective in this model ${ }^{22}$. The shortening of the QTCB interval with salbutamol in the present study, however, illustrates the limitations of Bazett's formula. Interestingly, the E-M window hardly changed with salbutamol despite drastic increases in heart rate. Similarly, significant decreases in heart rate associated with diltiazem did not affect the E-M window either.

The effect of temperature on the duration of the QT interval has been studied in detail in anaesthetized dogs ${ }^{48}$. A similar temperature dependency of the QT interval was observed in anaesthetized guinea-pigs: $-14.6 \mathrm{~ms}^{\circ} \mathrm{C}^{-1}(\mathrm{QTCB})$ in anaesthetized guinea-pigs versus $-14 \mathrm{~ms}^{\circ} \mathrm{C}^{-1}$ (QTcVDW) in anaesthetized dogs. However, the E-M window remained remarkably stable during cooling and even tended to increase slightly when cooling below $35.5^{\circ} \mathrm{C}$.

Taken together, the E-M window is, in contrast to the QTcB interval, a robust parameter that is minimally affected by changes in heart rate or body temperature.

\subsubsection{Limitations of the study}

No bio-analysis of plasma samples was performed, therefore results could not be presented as $\mathrm{x}$-folds of therapeutic concentrations and subsequently no safety margins could be determined. In literature some data on plasma concentrations and QTCB prolongation of reference drugs in anaesthetized guinea-pigs and dogs have been published ${ }^{6,24,45}$ that could be useful to put our results in perspective. The QTCB prolongations evoked by reference compounds observed in the current study are consistent with these previous reports.

\subsubsection{Conclusions}

The present work studied for the first time the $\mathrm{E}-\mathrm{M}$ window in anaesthetized guineapigs as a general risk marker for TdP in man. A decreased E-M window was consistently observed with drugs with high TdP risk, but not with drugs with low or no TdP risk. Furthermore, the E-M window was found to be a robust marker minimally affected by changes in heart rate or body temperature. These results suggest that the E-M window in anaesthetized guinea-pigs is an alternative risk marker for TdP in man that complements the traditional QT interval screening. 


\subsection{Acknowledgements}

The authors wish to thank Hanne Daems for her excellent technical assistance during the execution of the experiments. This work was financially supported by the Institute for Encouragement of Innovation by Science and Technology in Flanders (kmo-programma van het agentschap voor Innovatie door Wetenschap en Technologie, IWT). 


\section{References}

(1) Vohra J. The Long QT Syndrome. Heart Lung Circ. 2007;16 Suppl 3:S5-12.

(2) Darpo B. Spectrum of drugs prolonging QT interval and theincidence of torsades de pointes. Eur Heart J. 2001;3:K70-K80.

(3) Cavero I \& Crumb W. ICH S7B draft guideline on the non-clinical strategy for testing delayed cardiac repolarisation risk of drugs: a critical analysis. Expert Opin Drug Saf. 2005;4:509-530.

(4) Terrar DA, Wilson CM, Graham SG, Bryant SM \& Heath BM. Comparison of guinea-pig ventricular myocytes and dog Purkinje fibres for in vitro assessment of drug-induced delayed repolarization. $J$ Pharmacol Toxicol Methods. 2007;56:171-185.

(5) Testai L, Calderone V, Salvadori A, Breschi MC, Nieri P \& Martinotti E. QT prolongation in anaesthetized guinea-pigs: an experimental approach for preliminary screening of torsadogenicity of drugs and drug candidates. J Appl Toxicol. 2004;24:217-222.

(6) Yao X, Anderson DL, Ross SA, Lang DG, Desai BZ, Cooper DC et al. Predicting QT prolongation in humans during early drug development using hERG inhibition and an anaesthetized guinea-pig model. $\mathrm{Br} J$ Pharmacol. 2008; 154:1446-1456.

(7) Kagstrom J, Sjogren EL \& Ericson AC. Evaluation of the guinea pig monophasic action potential (MAP) assay in predicting drug-induced delay of ventricular repolarisation using 12 clinically documented drugs. J Pharmacol Toxicol Methods. 2007;56:186-193.

(8) Hoffmann P \& Warner B. Are hERG channel inhibition and QT interval prolongation all there is in drug-induced torsadogenesis? A review of emerging trends. J Pharmacol Toxicol Methods. 2006;53:87-105.

(9) Hondeghem LM. Thorough QT/QTc not so thorough: removes torsadogenic predictors from the T-wave, incriminates safe drugs, and misses profibrillatory drugs. J Cardiovasc Electrophysiol. 2006;17:337-340.

(10) Gintant GA. Preclinical Torsades-de-Pointes screens: advantages and limitations of surrogate and direct approaches in evaluating proarrhythmic risk. Pharmacol Ther. 2008;119:199-209. 
(11) Lawrence CL, Pollard CE, Hammond TG \& Valentin JP. Nonclinical proarrhythmia models: predicting Torsades de Pointes. J Pharmacol Toxicol Methods. 2005;52:46-59.

(12) van der Linde HJ, Van Deuren B, Somers Y, Loenders B, Towart R, Gallacher DJ. The electro-mechanical window: a risk marker for Torsade de Pointes in a canine model of drug induced arrhythmias. $\mathrm{Br} J$ Pharmacol. 2010;161:1444-1454.

(13) Boudoulas H, Geleris P, Lewis RP \& Rittgers SE. Linear relationship between electrical systole, mechanical systole, and heart rate. Chest. 1981;80:613-617.

(14) De Caprio L, Ferro G, Cuomo S, Volpe M, Artialo D, De luca N et al. (1984). QT/QS2 ratio as an index of autonomic tone changes. Am J Cardiol. 1984;53:818-822.

(15) Boudoulas H, Geleris P, Lewis RP \& Leier CV. Effect of increased adrenergic activity on the relationship between electrical and mechanical systole. Circulation. 1981;64:28-33.

(16) Boudoulas H, Sohn YH, O'Neill W, Brown R \& Weissler AM. The QT greater than QS2 syndrome: a new mortality risk indicator in coronary artery disease. Am J Cardiol. 1982;50:1229-1235.

(17) Airaksinen J, Ikaheimo M, Kaila J, Linnaluoto M \& Takkunen J. Systolic time intervals and the QT-QS2 interval in young female diabetics. Ann Clin Res. 1984;16:188-191.

(18) Chambers JB \& Ward DE. The QT and QS2 intervals in patients with mitral leaflet prolapse. Am Heart J. 1987;114:355-361.

(19) Vincent GM, Jaiswal D \& Timothy KW. Effects of exercise on heart rate, QT, QTc and QT/QS2 in the Romano-Ward inherited long QT syndrome. Am J Cardiol. 1991;68:498-503.

(20) Hauser DS, Stade M, Schmidt A \& Hanauer G. Cardiovascular parameters in anaesthetized guinea pigs: a safety pharmacology screening model. J Pharmacol Toxicol Methods. 2005;52:106-114. 
(21) Sakaguchi Y, Sugiyama A, Takao S, Akie Y, Takahara A \& Hashimoto K. (2005). Halothane sensitizes the guinea-pig heart to pharmacological $I_{\mathrm{Kr}}$ blockade: comparison with urethane anesthesia. $J$ Pharmacol Sci. 2005;99:185-190.

(22) Hamlin RL, Kijtawornrat A, Keene BW \& Hamlin DM. QT and RR intervals in conscious and anesthetized guinea pigs with highly varying RR intervals and given QTc-lengthening test articles. Toxicol Sci. 2003;76:437-442.

(23) Tabo M, Kimura K \& Ito S. Monophasic action potential in anaesthetized guinea pigs as a biomarker for prediction of liability for drug-induced delayed ventricular repolarization. J Pharmacol Toxicol Methods. 2007;55:254-261.

(24) Redfern WS, Carlsson L, Davis AS, Lynch WG, MacKenzie I, Palethorpe S et al. Relationships between preclinical cardiac electrophysiology, clinical QT interval prolongation and torsade de pointes for a broad range of drugs: evidence for a provisional safety margin in drug development. Cardiovasc Res. 2003;58:32-45.

(25) De Bruin ML, Pettersson M, Meyboom RH, Hoes AW \& Leufkens HG. AntiHERG activity and the risk of drug-induced arrhythmias and sudden death. Eur Heart J. 2005;26:590-597.

(26) Zicha S, Moss I, Allen B, Varro A, Papp J, Dumaine R et al. (2003). Molecular basis of species-specific expression of repolarizing $\mathrm{K}^{+}$currents in the heart. Am J Physiol Heart Circ Physiol. 2003;285:H1641-H1649.

(27) Lu Z, Kamiya K, Opthof T, Yasui K \& Kodama I. Density and kinetics of $\mathrm{I}_{\mathrm{Kr}}$ and $\mathrm{I}_{\mathrm{Ks}}$ in guinea pig and rabbit ventricular myocytes explain different efficacy of $\mathrm{I}_{\mathrm{Ks}}$ blockade at high heart rate in guinea pig and rabbit: implications for arrhythmogenesis in humans. Circulation. 2001;104:951-956.

(28) Ming Z \& Nordin C. Terfenadine blocks time-dependent $\mathrm{Ca}^{2+}, \mathrm{Na}^{+}$, and $\mathrm{K}^{+}$ channels in guinea pig ventricular myocytes. J Cardiovasc Pharmacol. 1995;26:761-769.

(29) Batey AJ \& Coker SJ. Proarrhythmic potential of halofantrine, terfenadine and clofilium in a modified in vivo model of torsade de pointes. $\mathrm{Br} \mathrm{J}$ Pharmacol. 2002; 135:1003-1012.

(30) Guns P-J, Teisman A, Van Ammel K, Towart R, Straetemans R, Bult H et al. Can retrospective analysis of preclinical cardiovascular safety data improve predictivity of future results? J Pharmacol Toxicol Methods. 2010;62:e29-e30. 
(31) Kodama I, Kamiya K \& Toyama J. Cellular electropharmacology of amiodarone. Cardiovasc Res. 1997;35:13-29.

(32) Hohnloser SH, Klingenheben $\mathrm{T}$ \& Singh BN. Amiodarone-associated proarrhythmic effects. A review with special reference to torsade de pointes tachycardia. Ann Intern Med. 1994;121:529-535.

(33) Mattioni TA, Zheutlin TA, Sarmiento JJ, Parker M, Lesch M \& Kehoe RF. Amiodarone in patients with previous drug-mediated torsade de pointes. Long-term safety and efficacy. Ann Intern Med. 1989;111:574-580.

(34) Lazzara R. Amiodarone and torsade de pointes. Ann Intern Med. 1989;111:549-551.

(35) van Opstal JM, Schoenmakers M, Verduyn SC, de Groot SH, Leunissen JD, van Der Hulst FF et al. Chronic amiodarone evokes no torsade de pointes arrhythmias despite QT lengthening in an animal model of acquired long-QT syndrome. Circulation. 2001;104:2722-2727.

(36) Yoshida H, Sugiyama A, Satoh Y, Ishida $Y$, Yoneyama M, Kugiyama et al. Comparison of the in vivo electrophysiological and proarrhythmic effects of amiodarone with those of a selective class III drug, sematilide, using a canine chronic atrioventricular block model. Circ J. 2002;66:758-762.

(37) Shah RR \& Hondeghem LM. Refining detection of drug-induced proarrhythmia: QT interval and TRIaD. Heart Rhythm. 2005;2:758-772.

(38) Merot J, Charpentier F, Poirier JM, Coutris G \& Weissenburger J. Effects of chronic treatment by amiodarone on transmural heterogeneity of canine ventricular repolarization in vivo: interactions with acute sotalol. Cardiovasc Res. 1999;44:303-314.

(39) Drouin E, Lande G \& Charpentier F. Amiodarone reduces transmural heterogeneity of repolarization in the human heart. $\mathrm{J} \mathrm{Am}$ Coll Cardiol. 1998;32:1063-1067.

(40) Patmore L, Fraser S, Mair D \& Templeton A. Effects of sparfloxacin, grepafloxacin, moxifloxacin, and ciprofloxacin on cardiac action potential duration. Eur J Pharmacol. 2000;406:449-452.

(41) Lu HR, Vlaminckx E, Van de Water A, Rohrbacher J, Hermans A \& Gallacher DJ. In-vitro experimental models for the risk assessment of antibiotic-induced QT prolongation. Eur J Pharmacol. 2007;577:222-232. 
(42) DiMasi JA \& Paquette C. The economics of follow-on drug research and development: trends in entry rates and the timing of development. Pharmacoeconomics. 2004;22:1-14.

(43) Ball P. Quinolone-induced QT interval prolongation: a not-so-unexpected class effect. J Antimicrob Chemother. 2000;45:557-559.

(44) Bloomfield DM, Kost JT, Ghosh K, Hreniuk D, Hickey LA, Guitierrez MJ et al. The effect of moxifloxacin on QTc and implications for the design of thorough QT studies. Clin Pharmacol Ther. 2008;84:475-480.

(45) Van Deuren B, Van Ammel K, Somers Y, Cools F, Straetemans R, van der Linde $\mathrm{HJ}$ et al. The fentanyl/etomidate-anaesthetised beagle (FEAB) dog: a versatile in vivo model in cardiovascular safety research. $J$ Pharmacol Toxicol Methods. 2009;60:11-23.

(46) Thomsen MB, Beekman JD, Attevelt NJ, Takahara A, Sugiyama A, Chiba K et al. No proarrhythmic properties of the antibiotics Moxifloxacin or Azithromycin in anaesthetized dogs with chronic-AV block. $\mathrm{Br} J$ Pharmacol. 2006;149:1039-1048.

(47) Magnano AR, Holleran S, Ramakrishnan R, Reiffel JA \& Bloomfield DM. Autonomic nervous system influences on QT interval in normal subjects. J Am Coll Cardiol. 2002;39;1820-1826.

(48) van der Linde HJ, Van Deuren B, Teisman A, Towart R \& Gallacher DJ. The effect of changes in core body temperature on the QT interval in beagle dogs: a previously ignored phenomenon, with a method for correction. Br J Pharmacol. 2008;154:1474-1481. 
Chapter 8

\section{General Discussion}

Daniel M Johnson • Najah Abi-Gerges • Paul GA Volders 


\subsection{General Discussion}

Over the last decades, drug-induced cardiac arrhythmias, including Torsades de Pointes (TdP) have become a major issue in drug development. Although (documented) episodes of TdP have a high impact for the individual patient, the estimated incidence is as low as 1 per 100,000 per year ${ }^{1,2}$. In 2005, the regulatory document ICH S7B was introduced with guidelines for a non-clinical testing strategy to assess the potential of a test substance to delay ventricular repolarization. These guidelines recommended the use of an ion-channel screen to investigate the effects of the test substance on the rapid component of the delayed-rectifier potassium channel ( $\mathrm{I}_{\mathrm{kr}}$-channel), as well as an in-vivo QT study in animals such as monkey, swine, dog, rabbit, ferret or guinea pig. The data obtained in these studies should then be combined and used to make an integrated risk assessment of the possible proarrhythmic capability of a test compound. In addition, these guidelines encouraged interested parties to develop proarrhythmic models and indices of proarrhythmic activity.

Although the current preclinical strategy, together with its clinical counterpart ICH E14, has been relatively successful in the attrition of unsafe drug candidates, cardiovascular safety remains one of the major reasons for the failure of compounds to reach the market. This is due to the fact that the mechanisms underlying TdP arrhythmia are not yet fully understood and the potential spectrum of side-effects of many drugs, including non-arrhythmic side-effects, is still incompletely explored. In addition, in the case of certain drugs post-marketing problems have occurred. For example, the COX2 inhibitor Vioxx, a non-steroidal anti-inflammatory drug (NSAID) that was approved by the FDA in May 1999 for a number of indications (including relief of the signs and symptoms of osteoarthritis, management of acute pain in adults, and for the treatment of menstrual symptoms) was voluntarily withdrawn by the manufacturer in 2004 due to safety concerns of an increased risk of cardiovascular events (including heart attack and stroke).

The major aim of this thesis was to provide further insights into the mechanisms of TdP arrhythmia. Further understanding of such mechanisms should lead to improved methods to distinguish safe medications from those that may lead to TdP and sudden cardiac death, and to improved prediction and prevention of this arrhythmia in the patient. Over the years numerous discussions have taken place to advance the field of cardiac safety pharmacology, with specific focus on reducing TdP risk ${ }^{3,4}$. In this thesis, a number of these issues have been addressed, including the use of proarrhythmia models to identify novel surrogate markers of TdP (e.g., temporal aspects of repolarization prolongation), and the role of myocardial $\mathrm{Ca}^{2+}$ handling in TdP induction. 


\subsection{Markers and Models for TdP}

Beat-to-beat variability of repolarization (BVR) has been proposed as a more predictive marker of TdP arrhythmia than repolarization duration alone ${ }^{5}$. BVR has previously been shown to be increased in a number of in-vivo preclinical proarrhythmic models, including the chronic-AV-block (CAVB) dog ${ }^{6}$ and the methoxamine-sensitized rabbit ${ }^{7}$. Additionally, increases in BVR have been observed in other experimental studies when animals were exposed to compounds that are known to cause $\mathrm{TdP}^{8}{ }^{8} 9$. Increases in BVR have also been seen in specific patient groups with an augmented arrhythmia risk ${ }^{10,}{ }^{11}$. Interestingly, a recent study also showed an increase of BVR in professional football players ${ }^{12}$. Although this study does not directly relate these increases to augmented arrhythmia propensity in this group, it is well known that strenuous exercise training is accompanied by ventricular hypertrophy, and this may be a substrate for sudden cardiac death in a small subset of these athletes ${ }^{13}$, who could be genetically predisposed.

One of the major aims of this thesis was to understand the underlying mechanisms of BVR, as until now these have remained largely elusive. In Chapter $\mathbf{2}$ we show that isolated canine myocytes can be used to examine the cellular electrophysiological effects of proarrhythmic compounds by using various techniques that are outlined in this chapter. We then use these techniques in Chapters $\mathbf{3}$ and 4 to delineate specific mechanisms of BVR, especially in the setting of intense $\beta$-adrenergic recptor ( $\beta A R$ ) stimulation (see section 8.3).

A recent interesting study from Nalos et al. ${ }^{14}$ tested various $I_{\mathrm{kr}}$ blockers, with different clinical proarrhythmic outcomes in five test systems, regarding cellular markers of arrhythmia prediction. In contrast to other published reports ${ }^{15}$, they conclude that isolated myocytes lack the specificity to identify proarrhythmic compounds. Nalos et al. ${ }^{14}$ show that in both remodeled ventricular myocytes from CAVB dogs and in unremodeled rabbit myocytes, moxifloxacin, a compound that has a relatively low proarrhythmic risk, can cause early afterdepolarizations (EADs). As a number of studies have indicated differential responses, at least in Purkinje fibres, between dog and rabbit preparations ${ }^{16,17}$ further investigation of these differences at the single myocyte level is warranted. Unfortunately, moxifloxacin has not been previously tested in isolated unremodeled canine myocytes and it would be of interest to see if moxifloxacin would cause EADs in this test system. These findings illustrate the importance of selecting the in-vitro test model that will have the most reliable translational outcome.

Despite the fact that the single canine myocyte may not be ideal for predicting arrhythmia in-vivo, this cell type can be used to provide major mechanistic insights, as has been carried out in this thesis, to aid in developing better arrhythmia prediction algorithms in vivo. 
The CAVB dog has been used as a model for proarrhythmic screening for a number of years, and as part of the work in this thesis we extend this by investigating the proarrhythmic capabilities of the combined ion-channel blocker AZD1305 (Chapter 6). AZD1305 was in clinical development for the termination of persistent atrial fibrillation. Apart from blocking $\mathrm{I}_{\mathrm{Kr}}$, this compound also inhibited $\mathrm{I}_{\text {NaLate }}$ and $\mathrm{I}_{\mathrm{CaL}}$ and by having this profile it had shown promise in a number of preclinical assays ${ }^{18,19}$. For example in the methoxamine-sensitized rabbit model, AZD1305 was devoid of any proarrhythmic actions despite causing large increases in repolarization times ${ }^{18}$. In our study we show that AZD1305 was much less proarrhythmic than the pure $\mathrm{I}_{\mathrm{Kr}}$ blocker dofetilide, despite causing similar ventricular repolarization prolongation. When TdP did occur, the arrhythmia(s) arose early during the 30-min infusion phase, when AZD1305 plasma concentrations were still rising. When no TdP arrhythmias were seen, BVR remained low, further illustrating the superiority of this parameter over QT prolongation alone. The road of clinical development of AZD1305 was unfortunately halted due to the fact that, as in the CAVB dog model, this compound led to TdP in a number of patients in a phase II clinical study ${ }^{20}$. We believe that if a compound shows more balanced ion-channel potencies than those of AZD1305 (i.e. similar inhibition of both inward and outward currents), the strategy of blocking these currents with a single agent could hold great therapeutic promise against cardiac arrhythmias without leading to TdP arrhythmia. These data highlight the importance of using sensitive proarrhythmic models such as the CAVB dog, before commencing costly clinical trials.

As the heart is an electrically-driven muscular pump, the link between its electrical and mechanical activity is of vital importance. An unsuspected abnormality in the ventricular contraction pattern of patients with prolonged repolarization due to congenital long QT syndrome (LQTS) and an association with syncope and cardiac arrest was demonstrated over 20 years ago ${ }^{21}$. However, only recently mechanical factors have been taken into consideration when discussing drug-induced arrhythmia ${ }^{22}$. Previously, Gallacher et al. ${ }^{8}$ elegantly demonstrated that in a canine model of long-QT1 (LQT1) syndrome, left-ventricular (LV) aftercontractions observed preceded the induction of TdP. After that study, van der Linde et al. ${ }^{23}$ showed that in anesthetized dogs under baseline conditions the difference between the end of the QT interval and the end of the mechanical activity of the heart (assessed on the LVpressure signal) was positive, indicating that the heart was still in systole when the electrical activity had ceased (a positive electro-mechanical (E-M) window). Interestingly, under conditions of combined $\mathrm{I}_{\mathrm{Ks}}$ blockade and $\beta A R$ stimulation, and prior to TdP arrhythmias in these animals, this relationship was reversed with completion of electrical activity taking substantially longer than the mechanical activity (negative E-M window). Furthermore, prevention of TdP with either the $\mathrm{Ca}^{2+}$ channel blocker verapamil, or the $\beta A R$ blocker atenolol, led to less negative E-M windows. In addition to these findings the disconnect between changes in the QT interval and the LV-pressure signal were illustrated when either heart rate or 
temperature were altered. These data demonstrated, for the first time, the possibility of using the E-M window for the prediction of drug-induced TdP, and its advantages over looking at alterations in QT time alone.

In Chapter 7 we extend the findings of van der Linde ${ }^{23}$ to an anesthetized guineapig model. Using a number of clinical-reference compounds with varying degrees of torsadogenic potential, we show that even though TdP was not induced in this model, the E-M window was able to discriminate compounds with known torsadogenic potential from compounds found to be safe, despite the fact that some of the latter agents alter repolarization times. Due to the cardiac electrophysiology of the guinea pig, with its strong 'repolarization reserve' due to slow deactivation of $\mathrm{I}_{\mathrm{Ks}}{ }^{24,25}$, no TdP arrhythmias were actually seen in this study with one agent alone, and therefore a direct link between TdP and negative E-M windows could not be established. A more recent study ${ }^{26}$, employing a strategy of a combined pharmacological assault, could however induce TdP arrhythmias in this model. This study illustrated that large negative E-M windows are a prerequisite for TdP induction in the anesthetized guinea pig. Taken together these findings are a first step in validation of this novel marker beyond the canine LQT1 model.

Since the publication of van der Linde et al. ${ }^{23}$, a number of groups have looked further into the E-M window, both in preclinical assays and also in its applicability to the clinical situation. Interesting work in isolated heart preparations illustrated that $\mathrm{I}_{\mathrm{Ks}}$ inhibition can lead to a reduction in the E-M window in isolated minipig hearts ${ }^{27}$. In addition, E-M windows remained more positive in mini-pig hearts than dog hearts, which may correlate with the resistance of the mini-pig heart to TdP arrhythmias. Finally, in dogs with chronic AV block from our own laboratory, preliminary experiments show that the E-M window is drastically reduced when compared to sinus-rhythm animals and this is further exaggerates before the induction of TdP (Figure 1). Further investigation of these changes is clearly warranted.

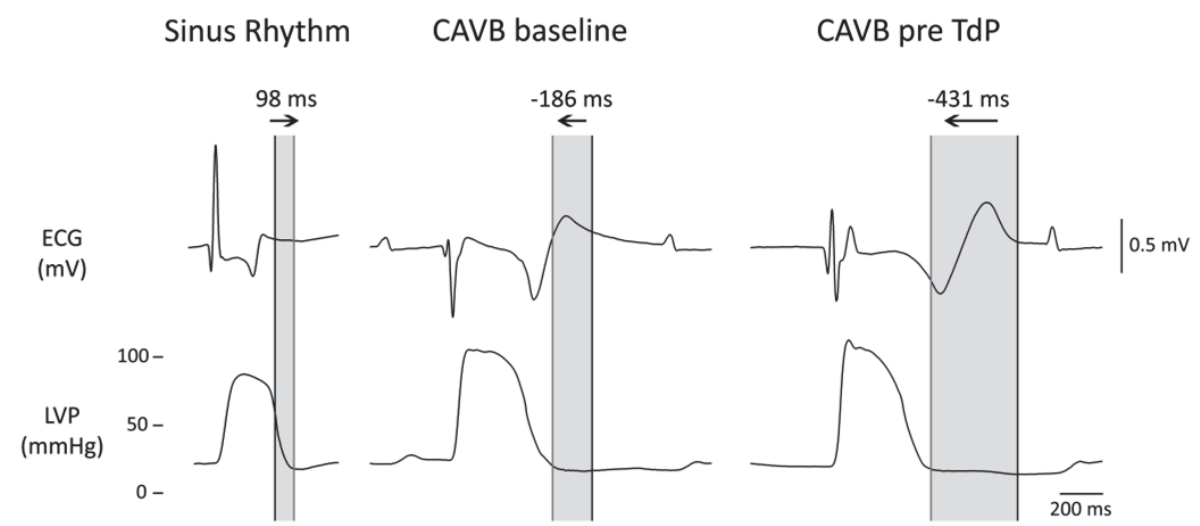

Figure 1: Changes in the E-M window after remodeling due to chronic AV block, and in the same animal just before dofetilide-induced aftercontractions and TdP are seen. 
Clinical data is also emerging that in patients with the congenital LQT syndrome, mean E-M windows were significantly more negative than in control patients $(-60 \pm 59$ ms versus $4 \pm 27 \mathrm{~ms})^{22}$. In addition, patients that were symptomatic had even more negative E-M windows when compared with asymptomatic LQT patients (-75 $\pm 68 \mathrm{~ms}$ versus $-39 \pm 36 \mathrm{~ms})$.

It appears therefore that negative E-M windows can set the stage for arrhythmia under certain conditions. Although it is likely that it is not the negative E-M window per se that is arrhythmogenic, it is likely that a negative window (meaning a situation with a long mechanical diastole during prolonged electrical systole) allows for arrhythmogenic aftercontractions to occur, which may provide a substrate for TdP, as demonstrated by Gallacher et al. ${ }^{8}$. Although the exact mechanisms linking negative E-M windows to arrhythmia generation are still unclear, they possibly involve a 'window of opportunity' for spontaneous SR $\mathrm{Ca}^{2+}$ release to occur in myocardium susceptible to $\mathrm{Ca}^{2+}$ overload, and/or the (re)activation of inward currents such as window $\mathrm{I}_{\mathrm{CaL}}, \mathrm{Na}^{+}-\mathrm{Ca}^{2+}$ exchange (NCX) current or stretchactivated currents. In this context, it will be interesting to ultimately determine whether aftercontractions are the consequence of spontaneous or $\mathrm{Ca}^{2+}$-induced $\mathrm{Ca}^{2+}$ release, and whether they activate inward mechano-sensitive currents to prolong repolarization further. With regard to the latter, a number of stretch-activated ion channels operate in the cardiac myocyte including $\mathrm{I}_{\mathrm{Na}}{ }^{28}$. A recent study ${ }^{29}$ interestingly showed that ranolazine, a drug with antianginal and antiarrhythmic (via (late) $I_{\mathrm{Na}}$ ) properties, also inhibits the mechanosensitivity of the $\mathrm{Na}^{+}$channel. Whether this property plays a role in this ranolazine's electropharmacological profile remains to be seen. Other stretch-activated channels are also operative in the cardiac myocyte ${ }^{22}$.

Further understanding of the presumed mechano-electrical feedback in TdP generation is crucial to establish novel antiarrhythmic strategies and for the development of safer medicinal compounds by pharmaceutical companies.

\subsection{Cellular Determinants of BVR}

One of the major themes of this $\mathrm{PhD}$ thesis is the investigation of the mechanistic basis of BVR and how alterations in this parameter may be arrhythmogenic, especially under conditions of sympathetic stimulation. BVR of the single myocyte most likely contributes to variability at the whole-heart level, although this is not the only governing factor ${ }^{30,31}$.

Under normal conditions the sympathetic nervous system is involved in mediating the so-called 'fight or flight' response. Under conditions of physiological stress the activity of this system is increased, and amongst effects on other organ systems, leads to profound increases of the contractile and electrical function of the heart. The link between altered sympathetic tone and arrhythmogenesis has long been established. 
For example, a study by Priori et al. ${ }^{32}$ showed that electrical stimulation of the left stellate ganglion (part of the intrinsic sympathetic nervous system) led to the formation of delayed afterdepolarizations (DADs) in the LV of anesthetized cats (see also Volders (2010) ${ }^{33}$ ). As BVR is an important marker of arrhythmia. It is important to improve insights into how sympathetic stimulation affects this marker, which may increase our understanding of the antiarrhythmic actions of beta blockers ${ }^{34,35}$ and left cardiac sympathetic denervation ${ }^{36,37}$.

In Chapter 3 our initial findings in single myocytes show that when $\mathrm{I}_{\mathrm{Ks}}$ is intact, sympathetic stimulation can rescue increased BVR induced by interventions that undermine 'repolarization reserve'. It has been demonstrated that this reserve consists, at least partly, of $\beta A R$-induced increases in $\mathrm{I}_{\mathrm{Ks}} 38-40$. Challenges to $\mathrm{I}_{\mathrm{Ks}}$ may thus undermine the repolarization reserve. For instance, as shown in Figure 2, the rescue of ATX-II-induced repolarization instability by isoproterenol (ISO) is undermined during $\mathrm{I}_{\mathrm{Ks}}$ blockade with HMR1556. Taken together, these data highlight the role of $I_{K s}$ in repolarization reserve.
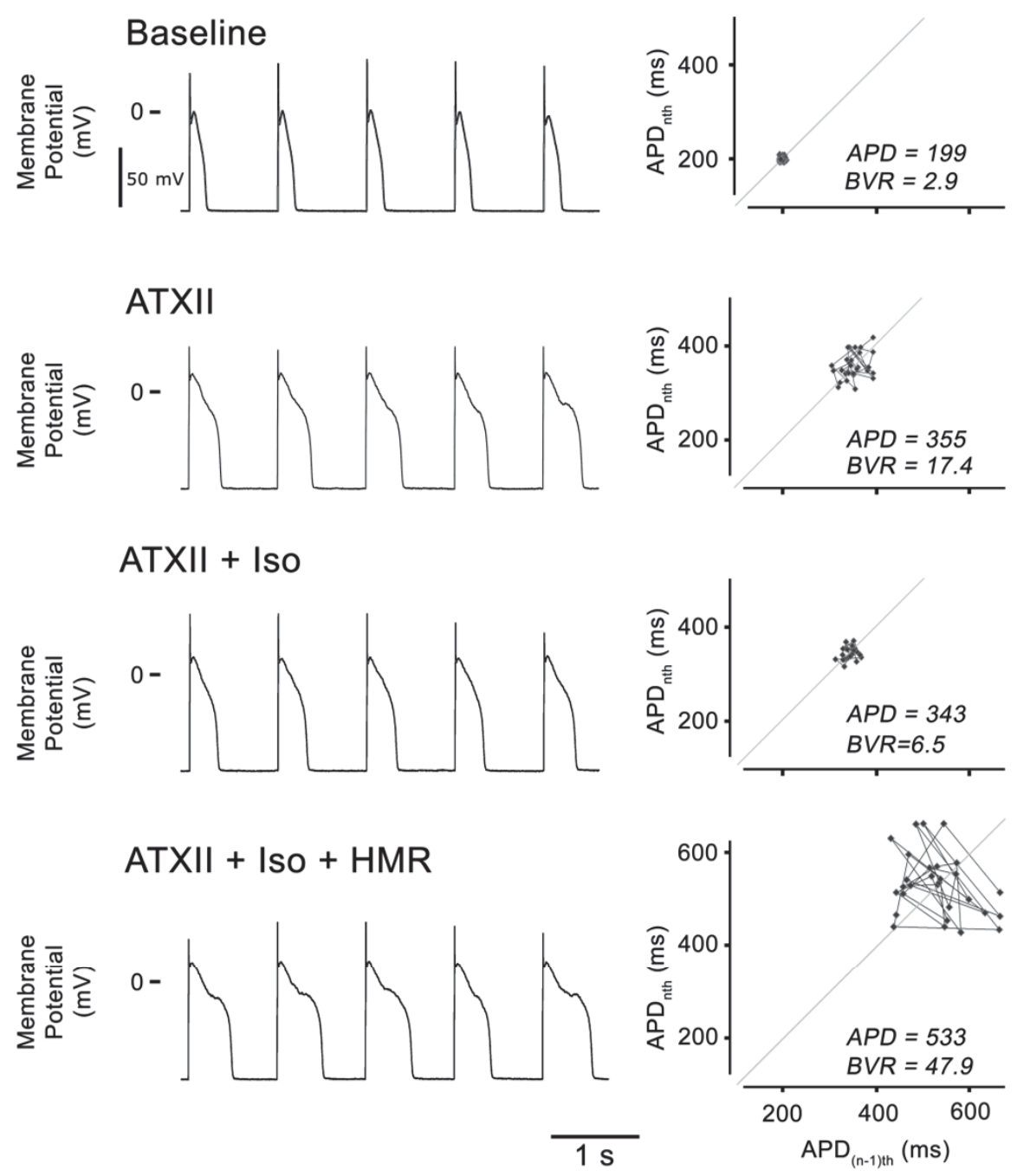

Figure 2: Inhibition of $I_{\mathrm{Ks}}$ by HMR1556 reverses the rescue of $\beta$ AR stimulation on ATX-IIinduced repolarization instability. 
Furthermore, in Chapter $\mathbf{3}$ we show that when $\mathrm{I}_{\mathrm{Ks}}$ is abolished pharmacologically and BVR increased substantially upon $\beta A R$ stimulation, this was followed by the generation of both DADs and early afterdepolarizations (EADs). Interestingly, intracellular $\mathrm{Ca}^{2+}$ chelation with BAPTA or inhibition of the NCX current reduced the BVR increase that was observed under these conditions, indicating that $\mathrm{Ca}^{2+}$ dependent currents were responsible. These studies were expanded in Chapter 4. In this chapter a combined experimental and computational approach was used to illustrate that increased BVR caused by the combination of $\mathrm{I}_{\mathrm{KS}}$ blockade and $\beta A R$ stimulation was largely due to interspersed action-potential prolongation due to the generation of presystolic DADs. We then went on to show that this phenomenon was caused by differences in $\mathrm{Ca}^{2+}$-dependent inactivation of the L-type $\mathrm{Ca}^{2+}$ current after presystolic spontaneous $\mathrm{Ca}^{2+}$ release. We showed that this interspersed actionpotential prolongation was also seen in an in-vivo canine model of LQT1, albeit on monophasic action potentials, and this contributed to increased dispersion of repolarization, measured by $T_{\text {peak }}-T_{\text {end }}$ intervals, which may ultimately play a role in arrhythmogenesis.

As BVR appears to be a better proarrhythmic marker than repolarization duration alone it is also important to understand the relationships between action-potential duration (APD) and BVR, because it may be hypothesized that when APD increases, BVR is also augmented. Figure 3 illustrates the relationships between these two parameters after different interventions. 

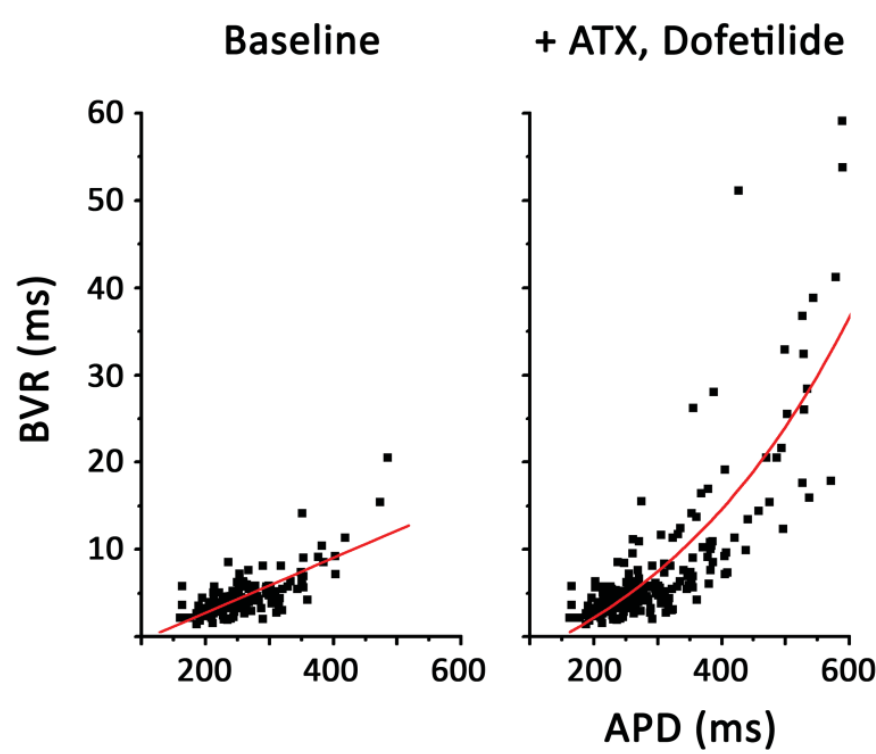

+ all $\mathrm{Ca}^{2+}$ handling alterations

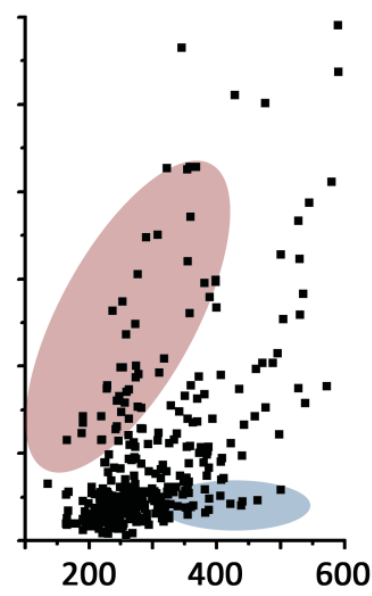

Figure 3: Relationship of BVR and action potential duration (APD) under baseline conditions and after various interventions in single canine myocytes. Left panel, baseline conditions; Middle panel, after APD prolongation by either $\mathrm{I}_{\mathrm{Kr}}$ blockade or late $\mathrm{I}_{\mathrm{Na}}$ augmentation. Finally, the right panel indicates that after various interventions that alter cellular $\mathrm{Ca}^{2+}$ handling this relationship can be substantially altered. The red 'cloud' indicates cells that were exposed to $\mathrm{I}_{\mathrm{KS}}$ inhibition together with $\beta A R$ stimulation (where BVR values are higher than would be expected for the given APD). The blue 'cloud' indicates cells that were exposed to agents that reduce $\mathrm{Ca}^{2+}$ loading, for example BAPTA and ryanodine (after exposure to $\mathrm{I}_{\mathrm{Ks}}$ inhibition and $\beta A R$ stimulation). Under these conditions BVR is lower than would be predicted from the APD values.

As can be seen in Figure 3, under baseline conditions the relationship between APD and BVR appears to be linear $\left(R^{2}=0.55\right)$, with BVR increasing at the same pace as APD. However when various interventions are applied to cause excessive prolongation of $A P D$, for example during $\mathrm{I}_{\mathrm{kr}}$ blockade, this relationship can be more reliably correlated with an exponential function $\left(R^{2}=0.72\right.$ for an exponential fit versus $R^{2}=0.67$ for a linear fit). These data indicate that under 'normal' conditions, the major influence on BVR is indeed APD, however when APD prolongation becomes excessive, disproportionate increases in BVR can be seen. When interventions are applied that directly alter $\mathrm{Ca}^{2+}$ handling within the myocytes and cause spontaneous $\mathrm{Ca}^{2+}$ releases, for example after $\mathrm{I}_{\mathrm{Ks}}$ block and $\beta A R$ stimulation, this balance is altered, with high levels of BVR observed despite the fact that the average APD is relatively low. On the other hand, when $\mathrm{Ca}^{2+}$ releases are completely blocked, for example with ryanodine, the relationship is tipped in the other direction, with large APDs being seen with relatively low BVR. Interestingly, similar data have been accrued from cells isolated from dogs with CAVB. In these cells the exponential relationship that was seen between APD and BVR could be linearized by using high doses of caffeine to cause complete block of the ryanodine receptor (Antoons et al., personal communication). 
Taken together, these data indicate that $\mathrm{Ca}^{2+}$ handling within the myocytes has a significant influence on alterations in BVR, and also suggest that there are possibilities to prolong APD without increasing BVR, should the correct balance be found. This has important implications for the proarrhythmic safety of medicinal compounds.

Recent computer simulations of BVR (Heijman et al., PhD thesis, Maastricht University, 2012) also support the notion that BVR is not only driven by APD and that stochastic gating strongly contributes to BVR. In that study an adapted computer model was developed to include such stochastic channel gating. This was achieved by stimulating individual channels and considering the probability of state transition, or by including specific Langevin terms in differential equations of 13 major ion channels and transporters. One of the major findings of this study was that APD prolongation strongly increased BVR, but this was not the only determinant of the exaggerated BVR seen under various conditions.

The results described in this $\mathrm{PhD}$ thesis also give important insights into other potential antiarrhythmic strategies. For example, an agent that can specifically prevent diastolic spontaneous $\mathrm{Ca}^{2+}$ release without affecting systolic $\mathrm{Ca}^{2+}$ release may have great utility under conditions of increased $\mathrm{Ca}^{2+}$ loading. Such an agent would also preserve systolic function, conserving cardiac output.

Altering $\mathrm{Ca}^{2+}$ handling has been found to reduce arrhythmic events in various models, including the CAVB dog, with a number of these indicating that reduced BVR plays an important role. In the CAVB dog model, the susceptibility to triggered arrhythmias has been related to a diminished repolarization reserve and to SR $\mathrm{Ca}^{2+}$ overload, due to electrical and structural remodeling ${ }^{41-44}$. Verduyn et al. ${ }^{45}$ showed that both flunarizine and ryanodine could prevent $\mathrm{I}_{\mathrm{kr}}$-block-induced TdP. Furthermore, a recent study by Oros et al. ${ }^{46}$ probed the antiarrhythmic mechanisms of flunarizine under these conditions and showed that this agent prevented dofetilide-induced TdP in these animals, which was also paralleled by BVR. This study went on to show that flunarizine decreased myocyte BVR and prevented the occurrence of EADs. In the same study, verapamil was also tested for its antiarrhythmic properties. Similarly to flunarizine, verapamil could prevent dofetilideinduced TdP, but interestingly neither a decrease in QT times nor (monophasic) APD was seen with this agent, despite a reduction in BVR. These data further support the idea that under certain conditions the relationship between BVR and APD can be dissociated. Perhaps suprisingly, Stams et al. ${ }^{47}$ has reported that K201, an agent that has been shown to reduce the amount of $\mathrm{Ca}^{2+}$ leak from the $\mathrm{SR}^{48}$, and that is under development for the prevention of atrial fibrillation, could neither suppress nor prevent dofetilide-induced TdP in the CAVB model. In fact, in the study of Stams et al. ${ }^{47}$, high doses of this agent alone led to drug-induced TdP, and this was accompanied by an increase in BVR. 
These findings may suggest that prevention of $\mathrm{Ca}^{2+}$ leak may not reduce BVR, however K201 not only inhibits $\mathrm{Ca}^{2+}$ leak but also inhibits multiple other ion channels which may influence the potential antiarrhythmic properties of this agent. Furthermore this agent prevents $\mathrm{Ca}^{2+}$ leak via a very specific mechanism, (stabilization of the ryanodine receptor via inhibition of calstabin2) and perhaps this intervention alone is not enough to prevent spontaneous $\mathrm{Ca}^{2+}$ release. Further studies in other models of TdP, such as in the LQT1 dog, may give some important insights into the reasons why this agent does not seem to have antiarrhythmic properties, despite its apparent favorable profile.

Interestingly, a number of studies have shown the applicability of CAMKII inhibition for prevention of arrhythmogenic EADs ${ }^{49}, 50$. Recently, Bourgonje et al. ${ }^{51}$ showed an increase in CaMKII phosphorylation in the proarrhythmic CAVB-dog model and a reduction of dofetilide-induced TdP after CAMKII inhibition with either W7 or KN-93. Work contained within this thesis provides further evidence that the strategy of CAMKII inhibition may be an alternative avenue for antiarrhythmic therapy, although clinically-safe specific inhibitors are not yet available to investigate this potential pharmacological breakthrough.

Altering NCX activity has also been proposed as an attractive antiarrhythmic target. Inhibiting forward mode NCX is a modulator of EADs, especially under conditions of $\mathrm{Ca}^{2+}$ overload. Our data support this notion and we have illustrated the utility of the NCX inhibitor SEA0400 in preventing excessive BVR in LQT1 stimulated myocytes (Chapter 3). Recent studies have substantiated these data in different models. Bourgonje et al. ${ }^{52}$ illustrated that SEA0400 is capable of reducing dofetilide-induced BVR (from 65 to $23 \mathrm{~ms}$ ) despite prolonged APD in single myocytes isolated from CAVB dogs, as well as abolishing EADs. Similarly, in-vivo this compound abolished dofetilide-induced TdP dose-dependently in these CAVB animals. Milberg et al. ${ }^{53}$ have shown that SEA0400 reduced proarrhythmia in a rabbit model of heart failure, although interestingly in this study, as opposed to the CAVB study, reductions in APD were observed. Currently, there is no definite answer as to how this strategy will fair in patients, and further research is warranted. However, these data are promising.

In the studies contained in this thesis, in which cellular BVR was assessed, only LV myocytes were used. Interestingly, at the whole heart level, at least in the CAVB dog, only LV BVR appeared to be predictive of arrhythmia formation ${ }^{30}$. In Chapter 5 of this thesis we show that there are specific regional differences in the response to $\beta A R$ stimulation in the LV versus the right ventricle (RV). We demonstrate that there is an enhanced responsiveness of the RV to $\beta A R$ stimulation which results from higher CAMP elevation in the cytoplasm due to a decreased degradation by PDE3 and PDE4 in the RV compared to the LV. We also demonstrate that even after $\beta A R$ stimulation the pressures in the RV are still much lower than those in the LV. It is well known that in-vivo the LV operates at much higher pressures than the RV. If we 
align this with other findings in this thesis, namely that spontaneous $\mathrm{Ca}^{2+}$ releases are one of the mechanisms behind increased BVR, we could speculate that the increased total $\mathrm{Ca}^{2+}$ loading in the LV contributes significantly to BVR in this ventricle. In the study of Gallacher et al. ${ }^{8}$ EADs only appeared on the LV MAP recordings at the endocardial surface, and not on the LV epicardium or in the RV, which corroborates results from canine LV-wedge studies reported by Katra and Laurita ${ }^{54}$ showing a predominant endocardial origin of triggered activity model of drug-induced LQT1 syndrome plus ISO. Previous studies have illustrated that both $\mathrm{I}_{\mathrm{Ks}}$ and $\mathrm{I}_{\mathrm{TO}}$ are larger in the RV when compared to the LV ${ }^{55}$, and therefore this inferior 'repolarization reserve' in the LV may be a determinant of exaggerated BVR in the LV. Investigation of how alterations of non-ionic targets, such as specific PDEs, change BVR and proarrhythmic outcomes is clearly needed.

\subsection{I mplications for Cardiac Safety Pharmacology and Future Directions}

Although it is well-known that inhibition of $\mathrm{I}_{\mathrm{Kr}}$ is not the only reason why druginduced TdP can occur, the ICH S7B guideline only recommends screening compounds against this ion channel, together with an in-vivo QT assay. In the work presented in this thesis we have shown, in agreement with previous studies ${ }^{30,56}$ that safe prolongation of repolarization is possible and that that increases in repolarization times alone do not necessarily lead to the occurrence of afterdepolarizations and proarrhythmia. In addition, we have further validated the utility of BVR as a proarrhythmic marker in vivo and have given further insights into the mechanisms involved in BVR and arrhythmia generation under conditions of $\beta A R$ stimulation and the importance of $\mathrm{I}_{\mathrm{Ks}}$ in preventing excessive BVR. Previous work has clearly shown that under normal conditions $I_{\mathrm{Ks}}$ has minimal effects on repolarization times under baseline conditions ${ }^{38,39}$, however its role becomes prominent during sympathetic stimulation or when repolarization is impaired by other factors (e.g., $\mathrm{I}_{\mathrm{Kr}}$ blockade ${ }^{57}$ ). Recently, it has also been shown that this current has antiarrhythmic properties in a rabbit model of ischemia/reperfusion ${ }^{58}$. Towart et al., ${ }^{59}$ have also shown that a number of compounds that were thought to be safe from the $\mathrm{I}_{\mathrm{kr}}$ screen and other invitro action potential assays, were actually highly proarrhythmic due to the fact that they had potent activity against $\mathrm{I}_{\mathrm{Ks}}$, and for this reason it is proposed that screening against this ion channel should be carried out in the early stage of drug discovery. Therefore, it is clear that potential effects on $\mathrm{I}_{\mathrm{Ks}}$ should also be considered when assessing the propensity of new chemical entities to cause arrhythmia. Data contained within this thesis further illustrate the need for such screening programs.

These data also bring into question the reasoning that the proarrhythmic screening of new compounds occurs only under baseline, normal healthy conditions. For example, APD assessment in-vitro is nearly always carried out in tissue from healthy animals using physiological salt solutions, and in QT assessments healthy animals are used. Contrary to this, the majority of pharmaceuticals are given to patients that are 
not healthy, meaning that their response to the drug may be altered. In addition, a number of other factors will also influence the proarrhythmic potential of novel agents in the clinical situation, including genetic disposition (either with regards to drug metabolism or (silent) mutations in cardiac proteins) and drug-drug interactions. For example, pharmacokinetic interactions with drugs known to inhibit cytochrome P450 isoenzymes (among them CYP3A4 and CYP2D6) may enhance the torsadogenic potential of agents normally metabolized by these enzymes CYP3A4 activity itself can be inhibited by a wide variety of drugs including some macrolide antibiotics, ketoconazole and related antifungals, cimetidine, protease inhibitors, and amiodarone. In addition, other factors, including age, smoking, hepatic disease, genetic polymorphisms and even grapefruit juice may lead to CYP3A4 inhibition.

It is therefore essential to examine the proarrhythmic potential of chemical entities in disease models and also at supratherapeutic doses. In this thesis we demonstrate that the proarrhythmic potential of AZD1305 could be detected in a proarrhythmic model, despite appearing safe from various preclinical assays. This compound did indeed cause TdP arrhythmias in selected patients. In addition to the use of proarrhythmic models for screening, consideration should also be given to testing compounds under altered physiological conditions, for example during sympathetic stimulation or conditions of altered electrolytes ${ }^{60}$. Over recent years development of patient-specific human induced pluripotent stem cells (iPSCs) and human embryonic stem cell-derived cardiomyocytes (hESC-CM) have been introduced as new paradigm for modeling human disease and for individualizing drug testing ${ }^{61,62}$. However, a recent study from Jonsson et al. ${ }^{63}$ has shown that the hESC-CM system has a number of weaknesses with regards to their electrophysiological phenotype, and consequently their potential as a safety pharmacology model. Therefore, much work remains to be done to fully understand the usefulness of stem-cell technology in cardiac safety pharmacology, and it will only be when these milestones have been reached before such systems will be widely adopted by the community.

In addition, we present a novel marker of proarrhythmia, the E-M window, in a small animal model, which appears to be another more reliable marker of TdP than QT prolongation alone. Interestingly the detection of adverse effects on cardiac contractility is also emerging in safety-pharmacology studies ${ }^{64}$. Various publications have described in-vitro ${ }^{65}$ and in-vivo models ${ }^{66-68}$ for assessing cardiac contractile parameters for safety assessment and in a number of pharmaceutical companies telemetry studies recording LV pressures during drug administration are routinely carried out. The integration of both electrical and mechanical recordings in one model has some clear advantages, including reducing the number of animal experiments required for drug development. Furthermore the integration of such parameters in a small animal model, may mean that these studies can be carried out at an earlier stage of drug discovery to ensure only the safest drug candidates are pursued. 
Finally, in Chapter $\mathbf{4}$ of this thesis, a combined experimental and computational approach is applied. Recently the use of computer modeling in safety pharmacology has received increasing attention, especially in the context of cardiac action potential modeling. A number of action potential models are currently available and these models are amongst some of the most detailed models in systems biology ${ }^{69}$. A recent example of such an approach in the safety pharmacology arena came from Davies et al. ${ }^{70}$, who developed an in-silico canine AP model to provide an assessment of the effect of a compound on the myocyte APD using concentrationeffect curve data from a panel of five cardiac ion channels. Uniquely this model fitted traces from 19 various in-vitro cellular experiments, with various ion-channel conductances, meaning that some variability was introduced into the model. In this model differential responses were seen in the 19 different 'cells' potentially explaining some of the observed variation in reaction to drugs. This work is a first step in developing and integrating multiple models to represent variability that may occur in patient populations. A number of other approaches have also been applied to integrate computer modeling into cardiac safety studies ${ }^{71-73}$. In this thesis computer modeling was used primarily to direct our experiments in the 'wet laboratory' and in addition it allows us to specifically analyze multiple parameters simultaneously (e.g., $\mathrm{I}_{\mathrm{Ks}}, \mathrm{I}_{\mathrm{CaL}}$ and $\mathrm{Ca}^{2+}$ transients) which is not currently possible in an experimental set-up. In addition modeling allows for specific targeting of proteins or ion channels, which, due to compound specificity issues, is not always the case in an experimental set-up. Over the next decades computer modeling will play an increasing role in providing such important information.

One of the major questions remaining is how much validation of a novel arrhythmia marker is required before it is adopted by regulatory agencies, and ultimately pharmaceutical companies. For example, in-vivo BVR was proposed as a marker for proarrhythmia back in 2004. However, at least to our knowledge, it has not been commonly adopted as a routine screening strategy. Whether this will also be the case for other proarrhythmic markers such as the E-M window remains to be seen. Also, the question is raised whether guidelines, such as the ICH S7B, need to be updated in the light of recent data such as those contained in this thesis. There is still a need for intense clinical investigation of the link between such markers and arrhythmogenesis, which may aid in decision-making. Recently a large multicentre clinical study has been commenced that will investigate current shortcomings in sudden cardiac death risk stratification and answer several related research questions, including the utility of BVR. The initial patient recruitment is underway and was expected to be completed by July 2012, with follow-up expected to end in September $2014^{74}$. The results of this trial and consequent actions are highly anticipated. 


\section{References}

(1) Wysowski DK, Bacsanyi J. Cisapride and fatal arrhythmia. $N$ Eng/ J Med. 1996;335:290-1.

(2) Darpo B. The thorough QT/QTc study 4 years after the implementation of the ICH E14 guidance. Br J Pharmacol. 2010;159:49-57.

(3) Bass A, Valentin JP, Fossa AA, Volders PG. Points to consider emerging from a mini-workshop on cardiac safety: assessing torsades de pointes liability. J Pharmacol Toxicol Methods. 2007;56:91-4.

(4) Bass AS, Darpo B, Breidenbach A, Bruse K, Feldman HS, Garnes D, Hammond T, Haverkamp W, January C, Koerner J, Lawrence C, Leishman D, Roden D, Valentin JP, Vos MA, Zhou YY, Karluss T, Sager P. International Life Sciences Institute (Health and Environmental Sciences Institute, HESI) initiative on moving towards better predictors of drug-induced torsades de pointes. Br J Pharmacol. 2008;154:1491-501.

(5) Thomsen MB, Volders PG, Beekman JD, Matz J, Vos MA. Beat-to-Beat variability of repolarization determines proarrhythmic outcome in dogs susceptible to drug-induced torsades de pointes. J Am Coll Cardiol. 2006;48:1268-76.

(6) Oros A, Beekman JD, Vos MA. The canine model with chronic, complete atrio-ventricular block. Pharmacol Ther. 2008;119:168-78.

(7) Jacobson I, Carlsson L, Duker G. Beat-by-beat QT interval variability, but not QT prolongation per se, predicts drug-induced torsades de pointes in the anaesthetised methoxamine-sensitized rabbit. J Pharmacol Toxicol Methods. 2011;63:40-6.

(8) Gallacher DJ, Van de Water A, van der Linde H, Hermans AN, Lu HR, Towart $\mathrm{R}$, Volders PG. In vivo mechanisms precipitating torsades de pointes in a canine model of drug-induced long-QT1 syndrome. Cardiovasc Res. 2007;76:247-56.

(9) Lengyel C, Varró A, Tábori K, Papp JG, Baczkó I. Combined pharmacological block of $\mathrm{I}_{\mathrm{Kr}}$ and $\mathrm{I}_{\mathrm{Ks}}$ increases short-term QT interval variability and provokes torsades de pointes. Br J Pharmacol. 2007;151:941-51. 
(10) Hinterseer M, Thomsen MB, Beckmann BM, Pfeufer A, Schimpf R, Wichmann $H E$, Steinbeck G, Vos MA, Kääb S. Beat-to-beat variability of QT intervals is increased in patients with drug-induced long-QT syndrome: a case control pilot study. Eur Heart J. 2008;29:185-90.

(11) Hinterseer M, Beckmann BM, Thomsen MB, Pfeufer A, Ulbrich M, Sinner MF, Perz S, Wichmann HE, Lengyel C, Schimpf R, Maier SK, Varro A, Vos MA, Steinbeck G, Kääb S. Usefulness of short-term variability of QT intervals as a predictor for electrical remodeling and proarrhythmia in patients with nonischemic heart failure. Am J Cardiol. 2010;106:216-20.

(12) Lengyel C, Orosz A, Hegyi P, Komka Z, Udvardy A, Bosnyak E, Trajer E, Pavlik G, Toth M, Wittmann T, Papp JG, Varro A, Baczko I. Increased short-term variability of the QT interval in professional soccer players: possible implications for arrhythmia prediction. PLoS One. 2011;6:e18751.

(13) Hart G. Exercise-induced cardiac hypertrophy: a substrate for sudden death in athletes? Exp Physiol. 2003;88:639-44.

(14) Nalos L, Varkevisser R, Jonsson MK, Houtman MJ, Beekman JD, van der Nagel R, Thomsen MB, Duker G, Sartipy P, de Boer TP, Peschar M, Rook MB, van Veen TA, van der Heyden MA, Vos MA. Comparison of the $I_{K r}$ blockers moxifloxacin, dofetilide and E-4031 in five screening models of pro-arrhythmia reveals lack of specificity of isolated cardiomyocytes. $\mathrm{Br} \mathrm{J}$ Pharmacol. 2012;165:467-78.

(15) Abi-Gerges N, Valentin JP, Pollard CE. Dog left ventricular midmyocardial myocytes for assessment of drug-induced delayed repolarization: short-term variability and proarrhythmic potential. Br J Pharmacol. 2010;159:77-92.

(16) Lu HR, Mariën R, Saels A, De Clerck F. Species plays an important role in drug-induced prolongation of action potential duration and early afterdepolarizations in isolated Purkinje fibers. J Cardiovasc Electrophysiol. 2001;12:93-102.

(17) Lu HR, Vlaminckx E, Gallacher DJ. Choice of cardiac tissue in vitro plays an important role in assessing the risk of drug-induced cardiac arrhythmias in human: beyond QT prolongation. J Pharmacol Toxicol Methods. 2008;57:1-8.

(18) Carlsson L, Andersson B, Linhardt G, Löfberg L. Assessment of the ion channel-blocking profile of the novel combined ion channel blocker AZD1305 and its proarrhythmic potential versus dofetilide in the methoxaminesensitized rabbit in vivo. J Cardiovasc Pharmacol. 2009;54:82-9. 
(19) Andersson B, Abi-Gerges N, Carlsson L. The combined ion channel blocker AZD1305 attenuates late $\mathrm{Na}$ current and $\mathrm{I}_{\mathrm{kr}}$-induced action potential prolongation and repolarization instability. Europace. 2010;12:1003-10.

(20) Rónaszéki A, Alings M, Egstrup K, Gaciong Z, Hranai M, Király C, Sereg M, Figatowski W, Bondarov $\mathrm{P}$, Johansson $\mathrm{S}$, Frison $\mathrm{L}$, Edvardsson N, Berggren A. Pharmacological cardioversion of atrial fibrillation--a double-blind, randomized, placebo-controlled, multicentre, dose-escalation study of AZD1305 given intravenously. Europace. 2011;13:1148-56.

(21) Nador F, Beria G, De Ferrari GM, Stramba-Badiale M, Locati EH, Lotto A, Schwartz PJ. Unsuspected echocardiographic abnormality in the long QT syndrome. Diagnostic, prognostic, and pathogenetic implications. Circulation. 1991;84:1530-42.

(22) Ter Bekke RM, Volders PG. Arrhythmogenic mechano-electric heterogeneity in the long-QT syndrome. Prog Biophys Mol Biol. 2012 Jul 24.

(23) van der Linde HJ, Van DB, Somers Y, Loenders B, Towart R, Gallacher DJ. The Electro-Mechanical window: a risk marker for Torsade de Pointes in a canine model of drug induced arrhythmias. $\mathrm{Br} J$ Pharmacol. 2010;161:1444-54.

(24) Jurkiewicz NK, Sanguinetti MC. Rate-dependent prolongation of cardiac action potentials by a methanesulfonanilide class III antiarrhythmic agent. Specific block of rapidly activating delayed rectifier $\mathrm{K}^{+}$current by dofetilide. Circ Res. 1993;72:75-83.

(25) Lu Z, Kamiya K, Opthof T, Yasui K, Kodama I. Density and kinetics of $\mathrm{I}_{\mathrm{Kr}}$ and $I_{\mathrm{Ks}}$ in guinea pig and rabbit ventricular myocytes explain different efficacy of $\mathrm{I}_{\mathrm{Ks}}$ blockade at high heart rate in guinea pig and rabbit: implications for arrhythmogenesis in humans. Circulation. 2001;104:951-6.

(26) Guns PJ, Johnson DM, Weltens E, Lissens J. Negative electro-mechanical windows are required for drug-induced Torsades de Pointes in the anesthetized guinea pig. J Pharmacol Toxicol Methods. 2012;66:125-34.

(27) Laursen M, Grunnet M, Olesen SP, Jespersen T, Mow T. Keeping the rhythm pro-arrhythmic investigations in isolated Göttingen minipig hearts. J Pharmacol Toxicol Methods. 2011;64:134-44.

(28) Beyder A, Rae JL, Bernard C, Strege PR, Sachs F, Farrugia G. Mechanosensitivity of Nav1.5, a voltage-sensitive sodium channel. J Physiol. 2010;588:4969-85. 
(29) Beyder A, Strege PR, Reyes S, Bernard CE, Terzic A, Makielski J, Ackerman $M J$, Farrugia G. Ranolazine decreases mechanosensitivity of the voltage-gated sodium ion channel $\mathrm{Na}(\mathrm{v}) 1.5$ : a novel mechanism of drug action. Circulation. 2012;125:2698-706.

(30) Thomsen MB, Verduyn SC, Stengl M, Beekman JD, de Pater G, van Opstal J, Volders PG, Vos MA. Increased short-term variability of repolarization predicts d-sotalol-induced torsades de pointes in dogs. Circulation. 2004;110:2453-9.

(31) Zaniboni M, Pollard AE, Yang L, Spitzer KW. Beat-to-beat repolarization variability in ventricular myocytes and its suppression by electrical coupling. Am J Physiol Heart Circ Physiol. 2000;278:H677-87.

(32) Priori SG, Mantica M, Schwartz PJ. Delayed afterdepolarizations elicited in vivo by left stellate ganglion stimulation. Circulation. 1988;78:178-85.

(33) Volders PG. Novel insights into the role of the sympathetic nervous system in cardiac arrhythmogenesis. Heart Rhythm. 2010;7:1900-6.

(34) Moss AJ, Zareba W, Hall WJ, Schwartz PJ, Crampton RS, Benhorin J, Vincent GM, Locati EH, Priori SG, Napolitano C, Medina A, Zhang L, Robinson JL, Timothy K, Towbin JA, Andrews ML. Effectiveness and limitations of betablocker therapy in congenital long-QT syndrome. Circulation. 2000;101:616-23.

(35) Schwartz PJ, Priori SG, Spazzolini C, Moss AJ, Vincent GM, Napolitano C, Denjoy I, Guicheney P, Breithardt G, Keating MT, Towbin JA, Beggs AH, Brink P, Wilde AA, Toivonen L, Zareba W, Robinson JL, Timothy KW, Corfield V, Wattanasirichaigoon D, Corbett C, Haverkamp W, Schulze-Bahr E, Lehmann $\mathrm{MH}$, Schwartz K, Coumel P, Bloise R. Genotype-phenotype correlation in the long-QT syndrome: gene-specific triggers for life-threatening arrhythmias. Circulation. 2001;103:89-95.

(36) Schwartz PJ, Priori SG, Cerrone M, Spazzolini C, Odero A, Napolitano C, Bloise R, De Ferrari GM, Klersy C, Moss AJ, Zareba W, Robinson JL, Hall WJ, Brink PA, Toivonen L, Epstein AE, Li C, Hu D. Left cardiac sympathetic denervation in the management of high-risk patients affected by the long-QT syndrome. Circulation. 2004;109:1826-33.

(37) Wilde AA, Bhuiyan ZA, Crotti L, Facchini M, De Ferrari GM, Paul T, Ferrandi C, Koolbergen DR, Odero A, Schwartz PJ. Left cardiac sympathetic denervation for catecholaminergic polymorphic ventricular tachycardia. $N$ Engl J Med. 2008;358:2024-9. 
(38) Volders PG, Stengl M, van Opstal JM, Gerlach U, Spätjens RL, Beekman JD, Sipido KR, Vos MA. Probing the contribution of $\mathrm{I}_{\mathrm{Ks}}$ to canine ventricular repolarization: key role for beta-adrenergic receptor stimulation. Circulation. 2003; 107:2753-60.

(39) Jost N, Virág L, Bitay M, Takács J, Lengyel C, Biliczki P, Nagy Z, Bogáts G, Lathrop DA, Papp JG, Varró A. Restricting excessive cardiac action potential and QT prolongation: a vital role for $\mathrm{I}_{\mathrm{Ks}}$ in human ventricular muscle. Circulation. 2005;112:1392-9.

(40) Varró A, Baczko I. Cardiac ventricular repolarization reserve: a principle for understanding drug-related proarrhythmic risk. $\mathrm{Br} J$ Pharmacol. 2011;164:14-36.

(41) Vos MA, Gorgels AP, Leunissen JD, Wellens HJ. Flunarizine allows differentiation between mechanisms of arrhythmias in the intact heart. Circulation. 1990;81:343-9.

(42) Volders PG, Sipido KR, Vos MA, Spätjens RL, Leunissen JD, Carmeliet E, Wellens $\mathrm{HJ}$. Downregulation of delayed rectifier $\mathrm{K}^{+}$currents in dogs with chronic complete atrioventricular block and acquired torsades de pointes. Circulation. 1999;100:2455-61.

(43) Sipido KR, Volders PG, de Groot SH, Verdonck F, Van de Werf F, Wellens HJ, Vos MA. Enhanced $\mathrm{Ca}^{2+}$ release and $\mathrm{Na} / \mathrm{Ca}$ exchange activity in hypertrophied canine ventricular myocytes: potential link between contractile adaptation and arrhythmogenesis. Circulation. 2000;102:2137-44.

(44) Dunnink A, van Opstal JM, Oosterhoff P, Winckels SK, Beekman JD, van der Nagel R, Cora Verduyn $S$, Vos MA. Ventricular remodelling is a prerequisite for the induction of dofetilide-induced torsade de pointes arrhythmias in the anaesthetized, complete atrio-ventricular-block dog. Europace. 2012;14:431-6.

(45) Verduyn SC, Vos MA, Gorgels AP, van der Zande J, Leunissen JD, Wellens HJ. The effect of flunarizine and ryanodine on acquired torsades de pointes arrhythmias in the intact canine heart. $J$ Cardiovasc Electrophysiol. 1995;6:189-200.

(46) Oros A, Houtman MJ, Neco P, Gomez AM, Rajamani S, Oosterhoff P, Attevelt NJ, Beekman JD, van der Heyden MA, Ver Donck L, Belardinelli L, Richard S, Antoons G, Vos MA; CONTICA investigators. Robust anti-arrhythmic efficacy of verapamil and flunarizine against dofetilide-induced TdP arrhythmias is based upon a shared and a different mode of action. $\mathrm{Br} \mathrm{J}$ Pharmacol. 2010;161:162-75. 
(47) Stams TR, Oros A, der Nagel R, Beekman JD, Chamberlin P, Dittrich HC, Vos MA. Effects of K201 on repolarization and arrhythmogenesis in anesthetized chronic atrioventricular block dogs susceptible to dofetilide-induced torsade de pointes. Eur J Pharmacol. 2011;672:126-34.

(48) Sacherer M, Sedej S, Wakuła P, Wallner M, Vos M, Kockskämper J, Stiegler P, Sereinigg $M$, von Lewinski D, Antoons G, Pieske B, Heinzel F; CONTICA investigators. JTV519 (K201) reduces sarcoplasmic reticulum $\mathrm{Ca}^{2+}$ leak and improves diastolic function in vitro in murine and human non-failing myocardium. Br J Pharmacol. 2012;167:493-504.

(49) Lu HR, Vlaminckx E, Van de Water A, Gallacher DJ. Calmodulin antagonist W-7 prevents sparfloxacin-induced early afterdepolarizations (EADs) in isolated rabbit purkinje fibers: importance of beat-to-beat instability of the repolarization. J Cardiovasc Electrophysiol. 2006;17:415-22.

(50) Qi X, Yeh YH, Chartier D, Xiao L, Tsuji Y, Brundel BJ, Kodama I, Nattel S. The calcium/calmodulin/kinase system and arrhythmogenic afterdepolarizations in bradycardia-related acquired long-QT syndrome. Circ Arrhythm Electrophysiol. 2009;2:295-304.

(51) Bourgonje VJ, Schoenmakers $M$, Beekman JD, Nagel RV, Houtman MJ, Miedema LF, Antoons G, Sipido K, de Windt LJ, van Veen TA, Vos MA. Relevance of calmodulin/CaMKII activation for arrhythmogenesis in the AV block dog. Heart Rhythm. 2012 Jul 27.

(52) Bourgonje VJA, Vos MA, Ozdemir S, Acsai K, Doisne N,Van Der Nagel R, Beekman HDM, Van Veen TAB, Sipido KR and Antoons G. Combined $\mathrm{Na} / \mathrm{Ca}$ exchanger and L-type calcium channel block by SEA-0400 suppresses Torsade de pointes arrhythmias with maintained haemodynamics. Cardiovasc Res. 2012;93 (suppl 1):S92-S127.P538.

(53) Milberg P, Pott C, Frommeyer G, Fink M, Ruhe M, Matsuda T, Baba A, Klocke R, Quang TH, Nikol S, Stypmann J, Osada N, Müller FU, Breithardt G, Noble $D$, Eckardt L. Acute inhibition of the $\mathrm{Na}^{+} / \mathrm{Ca}^{2+}$ exchanger reduces proarrhythmia in an experimental model of chronic heart failure. Heart Rhythm. 2012;9:570-8.

(54) Katra RP, Laurita KR. Cellular mechanism of calcium-mediated triggered activity in the heart. Circ Res. 2005;96:535-42.

(55) Volders PG, Sipido KR, Carmeliet E, Spätjens RL, Wellens HJ, Vos MA. Repolarizing $\mathrm{K}^{+}$currents $\mathrm{I}_{\mathrm{TO} 1}$ and $\mathrm{I}_{\mathrm{Ks}}$ are larger in right than left canine ventricular midmyocardium. Circulation. 1999;99:206-10. 
(56) Hondeghem LM, Carlsson L, Duker G. Instability and triangulation of the action potential predict serious proarrhythmia, but action potential duration prolongation is antiarrhythmic. Circulation. 2001;103:2004-13.

(57) Guérard NC, Traebert M, Suter W, Dumotier BM. Selective block of $I_{\mathrm{Ks}}$ plays a significant role in MAP triangulation induced by $\mathrm{I}_{\mathrm{Kr}}$ block in isolated rabbit heart. J Pharmacol Toxicol Methods. 2008;58:32-40.

(58) Guo X, Gao X, Wang Y, Peng L, Zhu Y, Wang S. I $\mathrm{K}_{\mathrm{Ks}}$ protects from ventricular arrhythmia during cardiac ischemia and reperfusion in rabbits by preserving the repolarization reserve. PLoS One. 2012;7:e31545.

(59) Towart R, Linders JT, Hermans AN, Rohrbacher J, van der Linde HJ, Ercken M, Cik M, Roevens $P$, Teisman A, Gallacher DJ. Blockade of the $I_{K s}$ potassium channel: an overlooked cardiovascular liability in drug safety screening? J Pharmacol Toxicol Methods. 2009;60:1-10.

(60) Yang T, Roden DM. Extracellular potassium modulation of drug block of $\mathrm{I}_{\mathrm{K} r}$. Implications for torsade de pointes and reverse use-dependence. Circulation. 1996;93:407-11.

(61) Moretti A, Bellin M, Welling A, Jung CB, Lam JT, Bott-Flügel L, Dorn T, Goedel A, Höhnke C, Hofmann F, Seyfarth M, Sinnecker D, Schömig A, Laugwitz KL. Patient-specific induced pluripotent stem-cell models for long-QT syndrome. N Engl J Med. 2010;363:1397-409.

(62) Itzhaki I, Maizels L, Huber I, Zwi-Dantsis L, Caspi O, Winterstern A, Feldman O, Gepstein A, Arbel G, Hammerman H, Boulos M, Gepstein L. Modelling the long QT syndrome with induced pluripotent stem cells. Nature. 2011;471:225-9.

(63) Jonsson MK, Vos MA, Mirams GR, Duker G, Sartipy P, de Boer TP, van Veen TA. Application of human stem cell-derived cardiomyocytes in safety pharmacology requires caution beyond hERG. $\mathrm{J} \mathrm{Mol} \mathrm{Cell} \mathrm{Cardiol.}$ 2012;52:998-1008.

(64) Force T, Kerkelä R. Cardiotoxicity of the new cancer therapeutics-mechanisms of, and approaches to, the problem. Drug Discov Today. 2008;13:778-84.

(65) Harmer AR, Abi-Gerges N, Morton MJ, Pullen GF, Valentin JP, Pollard CE. Validation of an in vitro contractility assay using canine ventricular myocytes. Toxicol Appl Pharmacol. 2012;260:162-72. 
(66) Norton K, Iacono G, Vezina M. Assessment of the pharmacological effects of inotropic drugs on left ventricular pressure and contractility: an evaluation of the QA interval as an indirect indicator of cardiac inotropism. J Pharmacol Toxicol Methods. 2009;60:193-7.

(67) Mooney L, Marks L, Philp KL, Skinner M, Coker SJ, Currie S. Optimising conditions for studying the acute effects of drugs on indices of cardiac contractility and on haemodynamics in anaesthetized guinea pigs. J Pharmacol Toxicol Methods. 2012;66:43-51.

(68) Johnson DM, Geys R, Lissens J, Guns PJ. Drug-induced effects on cardiovascular function in pentobarbital anesthetized guinea-pigs: Invasive LVP measurements versus the QA interval. J Pharmacol Toxicol Methods. 2012;66:152-9.

(69) Kohl P, Noble D. Systems biology and the virtual physiological human. Mol Syst Biol. 2009;5:292.

(70) Davies MR, Mistry HB, Hussein L, Pollard CE, Valentin JP, Swinton J, AbiGerges N. An in silico canine cardiac midmyocardial action potential duration model as a tool for early drug safety assessment. Am J Physiol Heart Circ Physiol. 2012;302:H1466-80.

(71) Mirams GR, Cui Y, Sher A, Fink M, Cooper J, Heath BM, McMahon NC, Gavaghan DJ, Noble D. Simulation of multiple ion channel block provides improved early prediction of compounds' clinical torsadogenic risk. Cardiovasc Res. 2011;91:53-61.

(72) O'Hara T, Rudy Y. Quantitative comparison of cardiac ventricular myocyte electrophysiology and response to drugs in human and nonhuman species. Am J Physiol Heart Circ Physiol. 2012;302:H1023-30.

(73) Zemzemi N, Bernabeu MO, Saiz J, Cooper J, Pathmanathan P, Mirams GR, Pitt-Francis J, Rodriguez B. Computational assessment of drug-induced effects on the electrocardiogram: from ion channel to body surface potentials. Br J Pharmacol. 2012 Sep 5.

(74) Seegers J, Vos MA, Flevari P, Willems R, Sohns C, Vollmann D, Lüthje L, Kremastinos DT, Floré V, Meine $M$, Tuinenburg A, Myles RC, Simon D, Brockmöller J, Friede T, Hasenfuß G, Lehnart SE, Zabel M; EUTrigTreat Clinical Study Investigators. Rationale, objectives, and design of the EUTrigTreat clinical study: a prospective observational study for arrhythmia risk stratification and assessment of interrelationship among repolarization markers and genotype. Europace. 2012;14:416-22. 


\section{Summary}

The major function of the heart is to continuously pump blood through the circulatory system of the entire body and hence careful regulation of this activity is essential. Synchronous pump function is driven by electrical signals, called action potentials. Under normal conditions these signals are generated and regulated by pacemaker cells within the sinoatrial (SA) node, which is located within right atrium. These electrical signals then enter the base of the ventricle at the Bundle of His and then follow the left and right bundle branches along the interventricular septum. The bundle branches then divide into an extensive system of Purkinje fibers that conduct the impulses at high velocity throughout the ventricles. This results in rapid depolarization of ventricular myocytes throughout both ventricles. In mammalian ventricular myocytes the action potential consists of a prolonged plateau phase where calcium ions can enter the myocytes of the ventricle, this triggers calcium induced calcium release, a process essential for excitation-contraction coupling. The global electrical signal of the heart is reflected in the electrocardiogram, and various parameters can be extrapolated from this signal, including the QT time which is utilized as a measure of repolarization duration.

The normal cardiac rhythm is very regular, with minimal fluctuation allowing for continual blood flow, however it can be altered to meet the oxygen demand by nervous impulses or by circulatory substances, including the adrenergic agonist adrenaline. Under certain conditions however the rhythm of the heart can become irregular, too fast (tachycardia) or too slow (bradycardia), or the beats of the atria and ventricle become unsynchronized. These arrhythmias can be caused by a multitude of both extrinsic and intrinsic factors, including genetic mutations (for example Long QT1 syndrome, catecholaminergic polymorphic ventricular tachycardia (CPVT)), cardiomyopathies and can also be drug-induced.

Over recent years drug-induced arrhythmias have received increased attention, in particular drug-induced Torsades de Pointes (TdP). This arrhythmia is a polymorphic ventricular tachycardia that can deteriorate into ventricular fibrillation and ultimately sudden cardiac death, and is normally seen in the context of a prolonged QT interval on the ECG. Although regulatory guidelines are in place to prevent compounds causing such arrhythmias getting to market, various pharmacological agents have been shown to cause this arrhythmia, and this has led to the withdrawal of a number of compounds. Currently prolongation of the QT interval and inhibition of one of the major ionic currents $\left(\mathrm{I}_{\mathrm{Kr}}\right)$ are used as surrogate markers that a compound may cause $\mathrm{TdP}$, however this strategy can still lead to both false negatives and false positives, a situation that is detrimental to drug development. 
The major aim of this thesis was to further understand mechanisms involved in the generation of this deadly arrhythmia, leading to improved prediction and prevention of this potentially deadly arrhythmia.

Chapter 1 is a general introduction, in which a background to cellular cardiac electrophysiology is given, as is an overview of the problem of drug-induced TdP and the current strategies for dealing with this issue at the preclinical stage of drug development. It is in this chapter where the concepts of beat-to-beat variability of repolarization (BVR) and the electro-mechanical (E-M) window are introduced. Finally in this chapter the aims and goals of the thesis are formulated.

In Chapter 2 we describe in detail a number of protocols for assessing changes in action potential duration in canine ventricular myocytes utilizing both optical imaging and electrophysiological techniques. We also present a protocol for assessing the occurrence of after-depolarizations and BVR at the level of the single cell. Both of these protocols can be utilized in cardiac safety pharmacology studies to predict the proarrhythmic potential of novel chemical entities and we illustrate how these protocols can reliably detect the electrophysiological effects of a number of reference compounds.

In Chapter 3 we use one of the techniques described in Chapter 2 to investigate how BVR can manifest itself at the single cell level. In this chapter isolated myocytes were used to investigate the importance of $\beta$-adrenergic receptor ( $\beta A R$ ) stimulation of $I_{K s}$ in the rescue of excessive increases in BVR following $\mathrm{I}_{\mathrm{Kr}}$ blockade and augmented late $I_{\mathrm{Na}}$. We show that both increases in action potential durations either by $\mathrm{I}_{\mathrm{kr}}$ blockade or augmentation of late $\mathrm{I}_{\mathrm{Na}}$ leads to increased BVR, and these increases can be counteracted by $\beta A R$ stimulation, most likely due to the concomitant increase in $\mathrm{I}_{\mathrm{Ks}}$. In addition we illustrate that an increased BVR corresponds with an increased occurrence of afterdepolarizations, further illustrating the usefulness of this parameter in prediction of arrhythmia. Interestingly we also show that under conditions of $\mathrm{I}_{\mathrm{Ks}}$ blockade and $\beta$ AR stimulation, similar to the LQT1 phenotype, BVR is exacerbated and this is also accompanied by the occurrence of afterdepolarizations. Both the increase in BVR and the afterdepolarizations can be abolished by chelation of intracellular calcium or by inhibition of the Sodium-Calcium exchanger current illustrating the possible utility of this strategy as a preventative antiarrhythmic treatment.

In Chapter 4 we further develop the findings in Chapter 3 by investigating the specific mechanisms of BVR under conditions of $I_{K s}$ blockade and $\beta A R$ stimulation. Using a variety of techniques we show that under these conditions spontaneous calcium releases (SCR) drive the increase in BVR by causing interspersed action potential prolongation. We illustrate that this is due to alterations in the inactivation of the L-type calcium channel after such calcium releases. A number of strategies, 
including CAMKII inhibition and treatment with magnesium were able to significantly reduce $B V R$, and hence we propose a number of preventative strategies in this chapter. These findings were extended beyond the single cell by investigating this phenomenon in both a whole animal model of LQT1 and in multicellular computer models. The findings shown in the single cell, also have relevance in both of these models, illustrating the fact that these data have significant translational value.

These data also illustrate the fact that delayed afterdepolarizations caused by SCRs can not only initiate arrhythmogenesis by causing triggered activity, but also contribute by increasing the temporal and spatial dispersion of repolarization.

Data included in Chapter 5 shows that there are specific regional differences in the response to $\beta A R$ stimulation in the left versus the right ventricle (RV). We demonstrate that there is an enhanced responsiveness of the RV to $\beta A R$ stimulation caused by higher CAMP elevation in the cytoplasm due to a decreased degradation by PDE3 and PDE4 in the RV compared to the LV. These data further illustrate interventricular differences which may be utilized to specifically intervene under various arrhythmic conditions. Further studies should concentrate on how this balance is altered under pathogenic conditions, which may aid in development of safer agents for prevention of arrhythmia

In Chapter 6 we move from the single cell to the whole animal .The chronic AV block dog model has been used for a number of years as a proarrhythmic model as in these animals structural and electrophysiological remodeling render these animals highly susceptible for TdP. In this chapter we use the novel multi ion channel blocker AZD1305 to further investigate the utility of BVR as a proarrhythmic marker in vivo. We show that AZD1305 has a lower proarrhythmic potential than the pure $\mathrm{I}_{\mathrm{kr}}$ blocker dofetilide in this model, despite both agents causing similar degrees of repolarization prolongation. Interestingly BVR of the left ventricular monophasic action potential was only increased after administration of dofetilide, further indicating the superiority of this proarrhythmic marker in prediction of TdP arrhythmia. Although AZD1305 did cause TdP in a subset of animals, these data further illustrate that the approach of inhibition of multiple (inward and outward) currents holds great promise as a safe antiarrhythmic strategy.

Chapter 7 investigates the utility of the E-M window as a predictive marker of TdP occurrence in a guinea pig model. The E-M window has recently been proposed as a novel marker for the prediction of TdP in a dog model of LQT1, and takes into account the temporal difference between then end of mechanical and electrical systole. We illustrate that when anaesthetized guinea pigs are challenged with agents that are known torsadogens, the E-M window becomes negative, with the electrical activity lasting longer than the mechanical activity. Interestingly when the electrical activity was 'safely' prolonged the E-M window did not become negative, 
illustrating the fact that this surrogate marker is superior and is more robust than looking at repolarization duration alone.

In the final chapter of this thesis, Chapter 8 , the main findings of this thesis are discussed together with both their possible significance for both drug development (prediction) and novel antiarrhythmic strategies (prevention) of drug induced TdP.

In conclusion the data provided within this thesis illustrates the importance of factors other than repolarization duration prolongation and the influence of $\beta A R$ stimulation and calcium handling in the manifestation of drug-induced arrhythmias. Insights are given into possible novel markers to improve the prediction of arrhythmia as well as possible antiarrhythmic mechanisms to prevent drug induced TdP. 


\section{Samenvatting}

De functie van het hart is hoofdzakelijk om continue bloed door het vatensysteem van het gehele lichaam te pompen, waarvoor een nauwkeurige regulatie noodzakelijk is. Gelijkmatig rondpompen wordt aangestuurd door elektrische signalen die actiepotentialen heten. Under normale omstandigheden ontstaan deze signalen iedere hartslag in pacemaker cellen in de sinusknoop, gelegen aan de bovenkant van de rechterboezem van het hart. Daarvandaan spreidt het elektrische signaal over de rechter- en linkerboezem voor een gesynchroniseerde contractie. Vervolgens komt het signaal via de atrioventrikulare knoop en de bundel van His in het interventrikulaire septum, waarvandaan het elektrische signaal vervolgens verder geleidt wordt door de linker en rechter bundeltakken. Hierna geleidt een complex netwerk van Purkinjevezels de elektrische impulsen met grote snelheid door de hartkamers voor een snelle en homogene depolarisatie van de hartspiermassa. Er bestaat een sterke hiërarchie voor de pacemakerfuncties in het hart, waarin meerdere delen van het hart de pacemakerfunctie kunnen overnemen als superieure pacemakers niet functioneren. Onder normale omstandigheden domineert de sinoatriale pacemaker het hartritme. Globale elektrische activiteit in het hart wordt weergegeven in het elektrocardiogram of hartfilmpje (ECG), waarin diverse parameters kunnen worden gemeten, zoals de QT tijd, die dient als maat voor de activatie- of repolarisatieduur van de hartkamers. Op het niveau van de hartspiercel wordt het elektrische signaal gedragen door transmembrane actiepotentialen. Deze hebben een verlengde plateaufase gedurende welke calcium ionen de cel binnengaan, die vervolgens weer leidt tot calcium-geïnduceerde calcium vrijgave, een proces dat essentieel is voor excitatie-contractie koppeling.

Het normale cardiale ritme is regelmatig, met slechts weinig fluctuatie, wat ervoor zorgt dat een continue bloedstroom mogelijk is. Indien nodig is het mogelijk het ritme aan te passen door middel van prikkels uit het autonome zenuwstelsel of door neurohumorale factoren zoals catecholamines, om aan een vergrote zuurstofvraag te kunnen voldoen. Onder bepaalde omstandigheden kan het hartritme onregelmatig worden: te snel (tachycardie), te traag (bradycardie), of de ritmes van boezems en kamers worden ontkoppeld. Deze ritmestoornissen kunnen door diverse ex- en intrinsieke factoren ontstaan, waaronder genetische mutaties (bijvoorbeeld het lange QT syndroom en catecholaminerge polymorfe ventriculaire tachycardie) en structurele cardiomyopathiën, maar ook als ongewenste bijwerking van bepaalde medicijnen.

Gedurende een aantal jaren hebben deze laatstgenoemde ritmestoornissen steeds meer aandacht gekregen, met name medicijn-geïnduceerde torsades de pointes (TdP). TdP is een polymorfe tachycardie die kan eindigen in kamerfibrilleren en vervolgens plotse hartdood, die veelal samen gaat met een verlenging van de QT tijd op het ECG. Welke mechanismen leiden tot TdP ritmestoornissen is nog steeds niet 
volledig bekend en ondanks dat er regels bestaan die moeten voorkomen dat stoffen op de markt komen die deze ritmestoornissen kunnen veroorzaken, hebben helaas diverse pharmacologische stoffen aantoonbaar geleid tot pro-aritmische bijwerkingen waarna een aantal van deze stoffen van de markt gehaald moesten worden. Een onvolledig begrip van deze ritmestoornis maakt dat de huidige indicatoren die TdP moeten voorspellen (QT verlenging en blokkade van een belangrijke ion stroom, $\mathrm{I}_{\mathrm{Kr}}$ ), zoals opgelegd door de regelgevende autoriteiten, verre van ideaal zijn, waaruit blijkt dat de voorspelling van TdP ritmestoornissen een van de grootste uitdagingen is voor de cardiale veiligheids farmacoloog. Daarom is het centrale doel in dit proefschrift dan ook om het mechanistische begrip van TdP uit te breiden, wat moet leiden tot een verbeterde voorspelling en het voorkomen van deze potentieel dodelijke ritmestoornis.

Hoofdstuk 1 is de algemene introductie waarin een achtergrond wordt gegeven van cardiale cellulaire elektrofysiologie en een overzicht van het probleem van medicijngeïnduceerde TdP. Ook de huidige strategieën die bestaan bij het preklinische deel van medicijn ontwikkeling worden besproken. In dit hoofdstuk worden verder de concepten van slag-op-slag variabiliteit van repolarisatie (BVR) en het elektromechanische $(E-M)$ "window" geïntroduceerd. Ten slotte worden de doelen van dit proefschrift geformuleerd.

In Hoofdstuk 2 beschrijven we gedetailleerde methoden om veranderingen in actiepotentiaal duur in kamercellen uit hondenharten te bestuderen met elektrofysiologische en optische beeldvorming technieken. We presenteren vervolgens een protocol waarmee het optreden van na-depolarisaties en de mate van BVR op het niveau van de geïsoleerde hartspiercel kan worden bepaald. Beide methoden kunnen bij cardiale veiligheids farmacologie studies gebruikt worden om het pro-aritmische potentiaal van nieuwe chemische stoffen te voorspellen op hartspiercel niveau en we tonen aan hoe deze protocollen de electrofysiologsiche effecten van een aantal referentie stoffen betrouwbaar detecteren.

In de studies voor Hoofdstuk 3 hebben we onderzocht hoe BVR zich manifesteert in ventriculaire hartspiercellen uit honden, gedurende medicijn-geïnduceerd lange QT type 1, 2 en 3 omstandigheden. Geïsoleerde hartspiercellen werden gebruikt om het belang van $\beta$-adrenerge receptor ( $\beta A R$ ) stimulatie van $I_{K s}$ gedurende bij het terugbrengen van overmatige toename van BVR, ontstaan door $\mathrm{I}_{\mathrm{kr}}$ blokkade en late $\mathrm{I}_{\mathrm{Na}}$ toename, aan te tonen. We tonen aan dat een toegenomen BVR overeenkomt met een toegenomen incidentie van vroege na-depolarisaties, wederom wijzend op de bruikbaarheid van deze parameter bij het voorspellen van ritmestoornissen. We tonen verder aan dat onder omstandigheden van $I_{K s}$ blokkade en $\beta A R$ stimulatie, zoals tijdens het lange QT fenotype, BVR is toegenomen en dat deze toename samen gaat met het optreden van na-depolarisaties. Zowel de toename in BVR als het optreden van na-depolarisaties kan ongedaan gemaakt worden door chelatie van 
intracellulair calcium of door remming van de natrium-calcium "exchanger" stroom, duidend op hun potentiaal als preventieve antiaritmische strategieën.

In Hoofdstuk 4 onderzoeken we specifieke mechanismen van BVR gedurende $\mathrm{I}_{\mathrm{Ks}}$ blokkade en $\beta A R$ stimulatie verder. Met behulp van verscheidene technieken tonen we aan dat onder deze omstandigheden spontane calcium vrijgave (SCR) zorgen voor de toename in BVR door wisselende actiepotentiaal verlenging. We tonen aan dat dit wordt veroorzaakt door wisselingen in de inaktivering van het L-type calcium kanaal na SCR. Diverse behandelingen, waaronder CaMKII inhibitie en toediening van magnesium, verminderden BVR. Vandaar dat we een aantal preventieve strategieën voorstellen gebaseerd op de resultaten uit dit hoofdstuk. We trekken onze resultaten door van de geïsoleerde hartspiercel naar het diermodel van LQT1 en multicellulaire computermodellen. We tonen aan dat late na-depolarisaties, veroorzaakt door SCR, niet alleen aritmieën kunnen veroorzaken via getriggerde activiteit, maar ook door temporele en spatiële dispersie van repolarisatie te vergroten.

Data uit Hoofdstuk 5 tonen aan dat er regionale verschillen bestaan in de respons op $\beta A R$ stimulatie in de linker (LV) en rechter (RV) hartkamer. Toegenomen gevoeligheid van de RV op $\beta A R$ stimulatie wordt veroorzaakt door een hogere cAMP concentratie in de myoplasma vanwege een toegenomen afbraak door PDE3 en PDE4 in de RV vergeleken met de LV. We tonen aan dat PDE3 en PDE4 ervoor zorgen dat CAMP gekompartimentaliseerd wordt waardoor locatie-specifieke verschillen tussen de hartkamers ontstaan bij $\beta A R$ stimulatie. Aangezien BVR alleen voorspellend is voor aritmieën wanneer deze gemeten wordt in de LV, kan deze kompartimentalisatie hier gedeeltelijk mee te maken hebben. Verder onderzoek is essentieel om het begrip over de mogelijke relaties tussen PDE's, CAMP signalen en BVR te vergroten. Verder kunnen toekomstige studies zich richten op hoe de BAR respons in de hartspiercel en de onderliggende verschillen in PDE verdeling veranderd worden onder pathologische cardiale omstandigheden, wat kan bijdragen tot verbeterd begrip van elektrische remodelering en de ontwikkeling van veiligere anti-aritmische medicijnen.

In Hoofdstuk 6 gaan we van de geïsoleerde cel naar het hele dier. Het hondenmodel met chronisch compleet atrioventriculair blok wordt al tientallen jaren gebruikt als pro-aritmisch model omdat deze dieren vanwege structurele en elektrische remodelering, zeer gevoelig zijn voor TdP. We gebruikten de nieuwe multi-kanaal blokker AZD1305 om de bruikbaarheid van BVR als pro-aritmische marker in vivo te onderzoeken. We tonen aan dat AZD1305 in dit model een lager pro-aritmisch potentiaal heeft dan de pure $\mathrm{I}_{\mathrm{Kr}}$ blokker dofetilide, ondanks dat beide stoffen een vergelijkbare verlenging van repolarisatie laten zien. Het is interessant te zien dat BVR van de LV monofasische actiepotentiaal alleen verlengt na toediening van dofetilide en dat dit, samen met andere kenmerken, verder wijst op de superioriteit 
van deze pro-aritmische marker bij het voorspellen van TdP ritmestoornissen. Ondanks dat AZD1305 leidde tot TdP in een subgroep, tonen deze data nog steeds aan dat het blokkeren van meerdere (in- en uitwaartse) ionstromen, veelbelovend is als antiaritmische strategie.

In Hoofdstuk 7 beschrijven we het gebruik van de E-M window als voorspellende marker voor het optreden van TdP in een cavia model. De E-M window werd recentelijk voorgesteld als nieuwe marker voor het voorspellen van TdP in een hondenmodel van LQT1 en het houdt rekening met het temporele verschil tussen het einde van de mechanische en elektrische systole. We tonen aan dat wanneer geanestheseerde cavia's worden blootgesteld aan bekende TdP opwekkers, de E-M window negatief wordt, waarbij de elektrische activiteit vaak (veel) langer duurt dan de mechanische activiteit. Opvallend is dat wanneer de elektrische activiteit "veilig" verlengt, de E-M window niet negatief wordt, hetgeen aantoont dat deze alternatieve marker beter en meer robuust is dan alleen repolarisatie duur.

In het laatste hoofdstuk van dit proefschrift, Hoofdstuk 8 , worden de voornaamste bevindingen uit dit proefschrift bediscussieerd, samen met hun mogelijke belang voor medicijn-ontwikkeling en veiligheids farmacologie (voorspelling) en nieuwe anti-aritmische strategieën (preventie) bij medicijn-geïnduceerde TdP.

Concluderend geven de resultaten uit dit proefschrift het belang aan van factoren anders dan verlenging van repolarisatie, de invloed van $\beta A R$ stimulatie en calcium verwerking in het optreden van medicijn-geïnduceerde ritmestoornissen. Er wordt inzicht gegeven in mogelijke, nieuwe markers die verbeterde voorspelling van deze ritmestoornis mogelijk maken, alsmede in mogelijke anti-aritmische mechanismen die medicijn-geïnduceerde TdP kunnen voorkomen. 


\section{Acknowledgements / Dankwoord}

Just as the cardiac action potential is a fine balance of interactions between various ion channels, the production of this $\mathrm{PhD}$ thesis has required many interactions with various people. Without the support and co-operation of these people this $\mathrm{PhD}$ thesis would not have been produced and therefore I would like to thank everyone who has helped me over the last years. Below is my attempt to name a few of these people but this is by no means exhaustive!

First and foremost I would like to thank my promotion team. Dr. Paul Volders, dear Paul, I am very grateful to you for giving me the opportunity to carry out my PhD thesis in your group, and for your continued support and input during this trajectory. I still remember our email exchanges back in 2005 from my desk at Merck, where I mention looking forward to the next four years of working in Maastricht for my PhD. It took a bit longer than that! Despite your busy clinical schedule, over the years we have had many scientific discussions (often on a Friday afternoon, lasting into the evening..) and late night email exchanges which have led to the completion of this thesis. Your knowledge of the literature continues to astound me and I hope that we can continue to collaborate in the future. Thanks for everything.

Dr. Najah Abi-Gerges, dear Najah, my industrial co-promotor. We have had many telephone calls over the years and you were always very welcoming during my visits to Alderley Park. During these meetings we would have long discussions together with the rest of the Safety Pharmacology team, and I would return to Maastricht with recharged batteries and new ideas. Without your continued provision of myocytes it would not have been possible to complete a number of studies contained within this thesis. It was also reassuring to hear when I wasn't the only one having problems getting recordings from these cells. I wish you all the best for the future.

I would like to thank Prof. Harry Crijns for acting as my promotor. During our progress meetings you were always extremely interested in the latest set of data and your enthusiasm on the subject was always infectious. A special word of thanks should also go here to Miriam Habex for secretarial assistance.

I would also like to thank Prof. Uli Schotten, Prof. Anton Gorgels, Prof. Tim Hammond, Prof. Marc Vos, and Prof. Thomas Unger for critically reading this thesis as part of the thesis assessment committee and for agreeing to be part of the corona at the defence of this thesis.

I am also indebted to my 'paranimfen' Monique de J ong and Roel Spätjens, not only for standing by me on the day of my defence but also for all the support they have given me over the years. 
Monique, your mantra of 'don't touch the green' is forever embedded in my brain. We have spent many, many hours in the operation room over the years and we have had many laughs! Positioning of MAP catheters was always an interesting experience and I'm sure when visitors came in during this process they thought that we were arguing, although this wasn't the case. Thanks for putting up with me on a Monday morning, making sure there was coffee on my arrival (especially on a Monday), and the late working nights. Thanks for our various discussions over numerous coffees and thank you for going through this thesis on numerous occasions to check the formatting issues!

Roel, your ability to successfully isolate myocytes time after time and your eye for making figures have been vital over the years! Thanks for all the solution preparation and the hours spent trying to patch. In addition your help on non-scientific issues and form filling was always appreciated. I wish you and your ladies all the best for the future!

A big thanks also to all members of the group over the years.

Jordi, I remember you coming in to the team as a Bachelor student and since then you have had a large impact on the work in this thesis (and very successfully defended your own!). I enjoyed our various discussions and your input on our joint projects. Without your software I think we would still be analyzing DADs... We really need to go for that beer soon! I wish you and Claire all the best in Essen/Heidelberg and beyond. Matthijs, good luck with the rest of your PhD research and combining this with your medical studies. I am really looking forward to seeing your results with the ECGI system. Rachel, all the best in your ongoing investigations on the EM window. I am very interested in seeing what the results will show. Miren, I wish you all the best back in Spain. I will always remember your Tamagotchi from the Nantes 2012 meeting! Marketa, it has been a while since you were in Maastricht but all the best for the future, I hope we will catch up again at future meetings. Inge, another ex-postdoc, I hope all is going well with your studies and family. Dirk, it is already a long time since you completed your thesis, but thanks for letting me get some invivo experience during some of your experiments. Helma, thanks for your assistance trying to get good myocyte staining. I look forward to seeing the latest data. Sandrine, thanks for preparing solutions and all the best for the family. Viola, again it has been a while since you left Maastricht for your Peruvian adventure, but thanks for the solutions. Annerie, I wish you all the best for the future. Lucien, all the best and Michael, i hope the combinatorial approach of patch clamping and modelling is going well and look forward to seeing your first results. Theo, it has also been while since your retirement, but thank you for your assistance in the OR at the beginning of this thesis. Finally Pol, although officially a member of CTC (whilst you were in Maastricht), thank you for all the time you spent in the OR with us and for your assistance. All the best for the future. 
I would also like to thank members of other departments who have assisted me over the years. Firstly to all the people who have shared an office with me. Veronica, Blanche, Esther, Anna and Georg. Thank you for putting up with my 'organised mess' of a desk and for listening to my gripes. Also thanks to Arne, Bart and Ali for the coffee breaks. I would also like to thank Joyce Suyk and her CPV team for looking after the animals that were used in these studies.

This thesis would not have been possible without the financial support from Astrazeneca. In addition to these financial aspects, I have also had numerous discussions with members of the safety pharmacology group in Alderley Park over the years. Both Drs. Jean-Pierre Valentin and Chris Pollard have provided interesting and important insights. I would also like to thank Dr. Michael Morton for carrying out some of the myocyte isolations and Dr. Will Redfern for providing Figure 2 in Chapter 1. Additionally I would like to thank Dr. Leif Carlsson, from AZ Sweden, for providing us with AZD1305 and for all the advice whilst carrying out the studies with this compound.

Over recent years I have also been collaborating with the group of Dr. David Gallacher, in particular the anaesthetized canine group headed by Henk van der Linde from Janssen Pharmaceuticals. Thank you for always being so welcoming on my visits to the Beerse site to gather that last bits of data for Chapter 4 of this thesis, and also for always providing data quickly and efficiently. I would also like to thank Bruno Van Deuren and Yves Somers for helping in carrying out these experiments and also in the labour intensive analysis.

I would like to thank Dr. Pieter-Jan Guns for providing a very pleasant working environment during my time at Bio-Plus and for collaboration on the electromechanical window studies. I wish you all the best at the University of Antwerp and also for your (and Valerie's) imminent arrival. I would also like to thank Dr. Rashmi Shah for providing the data contained in Table 1 of the introductory chapter of this thesis.

There have also been a number of collaborations with other members of the academic world. The groups of Prof. David Eisner and Prof. Andy Trafford from the University of Manchester provided important data and expertise that have been included in Chapter 4. In particular I would like to thank Dr. Dave Greensmith and Liz Bode for carrying out these experiments and also for your hospitality during my trip to Manchester.

Likewise I would like to thank the team of Prof. Rodolphe Fischmeister from INSERM for their collaborations leading to Chapter 5. I would like to especially thank Drs. Gregoire Vandecasteele, Jérôme Leroy and Cristina Molina (thanks for the late night FRET and patching and all the best back in Barcelona). We will get this 
article published in the short term. An additional thank you goes to Prof. Fischmeister for making the trip to Maastricht to be a member of the corona.

Finally I would like to thank the people who have contributed to this thesis in other ways. First of all, although now it seems like a lifetime ago, i would like to thank my old supervisors from Terling's Park, Drs. Zahid Ali, Karen Maubach and Kathy Sutton, for introducing me to the world of electrophysiology, and also for the support on my departure from Merck.

I would also like to thank all the people that I have met along the way during this process. Although you have now all left Maastricht you all helped me adjust to the move to Maastricht. Nick, Darryn, James, Apo, Eric, Nieves, Anna. Thanks for the nights in Take Five and Zondag and at the Quiz night, they made for a very pleasant distraction!

I would also like to thank my friends, in particular Warren, Will, Alex, Ed, Fahd and Elena for their continued support during this process and for their trips to continental Europe.

Janice and I an, I would also like to thank you for your support and hopefully after March $8^{\text {th }}$ you may finally understand some of the things I have been doing for the last years.

Ik wil ook mijn schoonfamilie bedanken. Lou and Jan, ik herinner me onze erste ontmoeting, wanneer Kim en ik op weg naar Pukkelpop waren een paar jaren geleden (6....). Vanaf dat moment hebben jullie mij altijd verwelkomd als een echt lid van de familie. Heel veel bedankt voor al jullie steun over de jaren. Ik denk dat jullie ook heel blij zijn dat het nu klaar is! Jeroen, Ik wens jou heel veel success met jouw carriere and jouw niet- 'smurfenhuis' in Antwerpen. It is a real shame you can't make it for the defence but you can put a bet on for me in Las Vegas! We will go for a celebratory beer soon!

Mum, without your support I would not be standing here today. Not just throughout this process but throughout everything I have done. When I told you I was considering a move to the Netherlands to carry out a PhD you have been nothing but supportive and have remained positive all the way through this process, even when I was less so! Being over in the Netherlands has meant that sometimes it has not been that easy to get back to Leeds but I hope that now this is finally completed that Kim, Hannah and I will be able to get over to the UK more often so that we can spend more time together. Thanks for everything. 
Finally Kim, if someone had told me when I started this thesis that when I finished it I would have a Flemish 'wife', a daughter and a house, I would have told them that they were crazy. However this is exactly how it has ended up and I could not be happier with the result! You know by now that I am not very good with words ('Dear Kim...' ), but without your endless support and love this thesis would never have come to fruition. There are many examples of this but one that sticks out it is whenever 'Dave' turned up at the house with myocytes at silly a.m. in the mornings. You never complained and then would even take me to work so that I could get on with experiments. You would then put up with me when I got back after a twenty something hour lab stint (not easy I think..). Now that my 'boekje' is finally finished, I cannot wait to start the next chapter of our lives together, now with our wonderful little girl, who makes me laugh every day (another reason to thank you!). Ik hou heel veel van jou. Dikke knuffel (still my favorite Dutch word).

Hannah, seeing your smile in the morning makes it all worth it and your presence has put everything into perspective. You really are the best daughter we could ever dream of, especially as your character blooms more and more each day. You have already shown your appreciation of beat-to-beat variability of repolarization by calming down when I read you a chapter of this thesis, so who knows what the future will bring $(-)$. I cannot wait to watch you grow up and I will try to be the best 'papa' I can be. 


\section{Curriculum Vitae}

\section{Daniel M. J ohnson}

Born in Leeds, UK on $13^{\text {th }}$ August

1999-2002 BSc Hons, Pharmacology. University of Newcastle-Upon-Tyne, UK

2002-2005 Staff Biologist, Neuroscience Research Centre, Merck Sharp and Dohme, Terlings Park, Harlow, Essex, UK

2005-2011 PhD, Dept. of Cardiology, Cardiovascular Research Institute Maastricht, The Netherlands

2011-2012 Safety Pharmacologist, Bio Plus Safety Pharmacology, Mol, Belgium

Daniel Johnson was born on the $13^{\text {th }}$ August 1979 in Leeds, United Kingdom. After obtaining his GCSEs and A-levels at Leeds Grammar School, Daniel moved to Newcastle and studied Pharmacology at the University of Newcastle-upon-Tyne.

On completion of his honours degree in 2002 he moved to work for Merck Sharp and Dohme, at the Neuroscience Research Centre in Harlow, Essex. During this time he used numerous in vivo and in vitro electrophysiological techniques and was involved in projects in various therapeutic areas, including Alzheimer's disease and pain research.

In 2005, Daniel started his $\mathrm{PhD}$ research as described in this thesis as part of collaboration between the Department of Cardiology and the Safety Assessment department of AstraZeneca in Alderley Park, United Kingdom. Daniel performed his PhD under the supervision of Prof. dr Harry Crijns and Dr. Paul Volders at Maastricht University and Dr. Najah Abi-Gerges at Alderley Park.

Between October 2011 and October 2012 Daniel worked for Bio-Plus Safety Pharmacology where he was involved in all aspects of running a small contract research laboratory. 


\section{List of Publications}

\section{Journal Articles}

J ohnson DM, Garrett EM , Rutter R , Bonnert TP, Gao YD, Middleton RE, Sutton KG; Functional Mapping of the Transient Receptor Potential Vanilloid 1 Intracellular Binding Site; Molecular Pharmacology 2006; 70(3):1005-1012.

Lecut C, Frederix K, Johnson DM, Deroanne C, Thiry M, Faccinetto C, Marée R, Evans RJ, Volders PGA, Bours V, Oury C; New Role for ATP-Gated P2X Ion Channels in the Control of Neutrophil Chemotaxis; Journal of Immunology 2009; 183(4):2801-9.

Johnson DM, Heijman J, Pollard CE, Valentin JP, Crijns HJGM, Abi-Gerges N, Volders PGA; $\mathrm{I}_{\mathrm{Ks}}$ Restricts Excessive Beat-to-Beat Variability of Repolarization during $\beta$-Adrenergic Receptor Stimulation; Journal of Molecular and Cellular Cardiology 2010; 48(1):122-30.

Johnson DM, Hussein L, Spätjens R, Valentin JP, Volders PGA, Pollard CE, Abi-Gerges N; Measuring Action Potential Generation in Isolated Canine Left Ventricular Midmyocardial Myocytes; Current Protocols in Pharmacology 2011; Unit 10.14 .

Johnson DM, de Jong MMJ, Crijns HJGM, Carlsson L, Volders PGA; Reduced Ventricular Proarrhythmic Potential of the Novel Combined Ion-Channel Blocker AZD1305 versus Dofetilide in Dogs with Remodeled Hearts; Circulation; Arrhythmia and Electrophysiology 2012; 5:201-209.

Guns PJ, Johnson DM, Van Op den Bosch J, Weltens E, Lissens J; The ElectroMechanical Window in Anaesthetized Guinea-Pigs: a New Marker for Torsade de Pointes Risk Screening.Br J Pharmacol. 2012; 66:689-701.

Guns PJ, J ohnson DM, Weltens E, Lissens J; Negative Electro-Mechanical Windows are Required for Drug-Induced Torsades de Pointes in the Anaesthetized Guinea Pig, Journal of Pharmacological and Toxicological Methods 2012; 66(2):125-34.

Johnson DM, Geys R, Lissens J and Guns PJ; Drug-Induced Effects on Cardiovascular Function in Pentobarbital Anaesthetized Guinea-Pigs: Invasive LVP Measurements versus the QA Interval. Journal of Pharmacological and Toxicological Methods 2012; 66(2):152-9. 
Johnson DM*, Heijman J*, Bode EF, Greensmith, D, van der Linde H, Abi-Gerges N, Eisner DA, Trafford A, Volders PGA; Diastolic Spontaneous Calcium Release from the Sarcoplasmic Reticulum Increases Beat-to-Beat Repolarization Variability in CalciumOverloaded Canine Ventricular Myocytes after $\beta$-Adrenergic Stimulation. Circ Res. 2013; 112(2):246-56.

Molina CE*, Johnson DM*, Mehel H, Spätjens R, Mika D, Algalarrondo V, Haj Slimane $Z$, Lechène $P$, Abi-Gerges $N$, Leroy $J$, Volders $P$, Fischmeister $R$, Vandecasteele G; Regional Specific Differences in $\beta$-Adrenergic Responses in Canine Left versus Right Ventricle: Role of Phosphodiesterases. Manuscript under revision.

Heijman J, Zaza A, Johnson DM, Rudy Y, Peeters RLM, Volders PGA, Westra RL; Computational analysis of Beat-to-Beat Variability of Repolarization Duration in the Canine Ventricular Myocyte, Manuscript in preparation.

\section{Abstracts}

Heijman J, Johnson DM, Westra RL, Volders PGA; Beat-to-Beat Variability of Repolarization Duration: Intrinsic to Native Myocytes, But Does it Feature in Ventricular Cell Models?, Biophysical Journal 2007; Supplement,285A.

Johnson DM, Heijman J, Pollard CE, Valentin JP, Abi-Gerges N, Volders PGA; Beatto-Beat Variability of Repolarization in Canine Ventricular Cell Models of LQT1, LQT2 and LQT3 syndromes, $31^{\text {st }}$ Annual Meeting of the European Working Group on Cardiac Cellular Electrophysiology, 2007, Manchester, UK.

Johnson DM, Heijman J, Pollard CE, Valentin JP, Abi-Gerges N, Volders PGA; Differential Responses of Beat-to-Beat Variability of Repolarization to $\beta$-Adrenergic Stimulation in Canine Ventricular Myocytes: $\mathrm{I}_{\mathrm{Ks}}$ Block versus $\mathrm{I}_{\mathrm{Kr}}$ block and Late $\mathrm{I}_{\mathrm{Na}}$ Augmentation, Heart Rhythm 2008; 5(5),S211.

Johnson DM, Pollard CE, Valentin JP, Crijns HJGM, Abi-Gerges N, Volders PGA; Forward $\mathrm{Na}^{+}-\mathrm{Ca}^{2+}$ Exchange Exaggerates Beat-to-Beat Variability of Repolarization Duration in a Cellular Model of Long-QT1 Syndrome, European Heart Journal 2009; 30 (Abstract Supplement),72.

Johnson DM, Heijman J, Volders PGA; Diastolic Spontaneous Calcium Release from the Sarcoplasmic Reticulum Provokes Beat-to-Beat Repolarization Instability in Calcium-Overloaded Myocytes, Heart Rhythm 2011; 8(5),S211. 
J ohnson DM*, Heijman J*, Bode EF, Greensmith, D, van der Linde H, Abi-Gerges N, Eisner DA, Trafford A, Volders PGA; Increased $\mathrm{I}_{\text {Cal }}$ Underlied Ventricular ActionPotential Prolongation After Spontaneous $\mathrm{Ca}^{2+}$ Release from the Sarcoplasmic Reticulum, Heart Rhythm 2011, 8(11),1823.

Heijman J, Zaza A, Johnson DM, Rudy Y, Peeters RLM, Volders PGA, Westra RL; Stochastic Ion-Channel Gating Contributes to Beat-to-Beat Variability of Repolarization and its Rate Dependence, Cardiovascular Research 2012; 93(1),S127.

Johnson DM, Lissens J, Guns PJ, Negative Electro-Mechanical Windows Are Required for Drug-Induced Torsades de Pointes in the Anaesthetized Guinea Pig, Safety Pharmacology Meeting 2012, Phoenix, Arizona.

Johnson DM, Geys R, Lissens J, Guns PJ, Assessment of Drug-Induced Effects on Cardiovascular Function in Pentobarbital Anaesthetized Guinea Pigs, Safety Pharmacology Meeting 2012, Phoenix, Arizona.

\section{Awards}

Travel Award from the Council of Basic Cardiovascular Research for attendance of ESC meeting in Barcelona, 2009. Poster Presentation.

Travel Award from the Council of Basic Cardiovascular Research for attendance of EWGCCE meeting in Nantes, 2012. Oral Presentation.

Junior Investigator Travel Award from the Safety Pharmacology Society for the attendance of Safety Pharmacology Society meeting in Phoenix, Arizona, 2012. Oral Presentation. 
